\title{
Cluster Structures on Double Bott-Samelson Cells
}

\author{
Linhui Shen $^{(D)}$ and Daping Weng ${ }^{(1)}$ \\ Michigan State University, 619 Red Cedar Road, East Lansing, MI, 48824, U.S.; E-mail: linhui @ math.msu.edu, \\ wengdap1@msu.edu.
}

Received: 30 July 2020; Revised: 25 April 2021; Accepted: 14 August 2021

Keywords: cluster varieties, double Bott-Samelson cells, Donaldson-Thomas transformation, Legendrian links

2020 Mathematics Subject Classification: Primary - 13F60, 14M15; Secondary - 14N35, 57K14

\begin{abstract}
Let $\mathrm{C}$ be a symmetrisable generalised Cartan matrix. We introduce four different versions of double Bott-Samelson cells for every pair of positive braids in the generalised braid group associated to $\mathrm{C}$. We prove that the decorated double Bott-Samelson cells are smooth affine varieties, whose coordinate rings are naturally isomorphic to upper cluster algebras.

We explicitly describe the Donaldson-Thomas transformations on double Bott-Samelson cells and prove that they are cluster transformations. As an application, we complete the proof of the Fock-Goncharov duality conjecture in these cases. We discover a periodicity phenomenon of the Donaldson-Thomas transformations on a family of double Bott-Samelson cells. We give a (rather simple) geometric proof of Zamolodchikov's periodicity conjecture in the cases of $\Delta \square \mathrm{A}_{r}$.

When $\mathrm{C}$ is of type A, the double Bott-Samelson cells are isomorphic to Shende-Treumann-Zaslow's moduli spaces of microlocal rank-1 constructible sheaves associated to Legendrian links. By counting their $\mathbb{F}_{q}$-points we obtain rational functions that are Legendrian link invariants.
\end{abstract}

\section{Introduction}

\subsection{Cluster Structures on Double Bott-Samelson Cells}

Bott-Samelson varieties were introduced by Bott and Samelson [BS58] in the context of compact Lie groups and were reformulated by Hansen [Han73] and Demazure [Dem74] independently in the reductive algebraic group setting. Bott-Samelson varieties give resolutions of singularities of Schubert varieties and have many applications in geometric representation theory. Webster and Yakimov [WY07] considered the product of two Bott-Samelson varieties and gave a stratification whose strata are parametrised by a triple of Weyl group elements and observed that a family of strata are isomorphic to double Bruhat cells introduced by Fomin and Zelevinsky [FZ99]. Lu and Mouquin [LM17] introduced a Poisson variety called generalised double Bruhat cells, which is defined by a conjugate class in a semisimple Lie group together with two $n$-tuples of Weyl group elements. Elek and Lu [EL19] further studied the special case of generalised Bruhat cells where one of the $n$-tuples was trivial and proved that their coordinate rings, as Poisson algebras, are examples of symmetric Poisson Cauchon-GoodearlLetzter (CGL) extension defined by Goodearl and Yakimov [GY18].

Motivated by the positivity phenomenon on double Bruhat cells, Fomin and Zelevinsky [FZ02] introduced a class of commutative algebras called cluster algebras. Fock and Goncharov [FG09a] introduced cluster varieties as the geometric counterparts of cluster algebras and conjectured that the

(C) The Author(s), 2021. Published by Cambridge University Press. This is an Open Access article, distributed under the terms of the Creative Commons Attribution licence (http://creativecommons.org/licenses/by/4.0/), which permits unrestricted re-use, distribution, and reproduction in any medium, provided the original work is properly cited. 
coordinate rings of cluster varieties admit canonical bases parametrised by the integral tropical set of their dual cluster varieties. The cluster structures on double Bruhat cells have been studied extensively in [BFZ05, FG06, GSV10].

In this article, we introduce a new family of varieties called double Bott-Samelson cells as a natural generalisation of double Bruhat cells and study their cluster structures.

Our generalisation goes in two directions: first, we extend the groups from semisimple types to Kac-Peterson groups, whose double Bruhat cells have been studied by Williams [Wil13]; second, we replace a pair of Weyl group elements $(u, v)$ by a pair of positive braids $(b, d)$, which we believe is a new construction. In particular, our double Bott-Samelson cells further generalise Lu and Mouquin's generalised double Bruhat cells associated to the identity conjugacy class [LM17] by dropping the additional data of partitioning the positive braids $b$ and $d$ as two $n$-tuples of Weyl group elements and extending the family to include the Kac-Peterson cases.

We present three versions of double Bott-Samelson cells, an undecorated one, $\operatorname{Conf}_{d}^{b}(\mathcal{B})$, and two decorated ones, $\operatorname{Conf}_{d}^{b}\left(\mathcal{A}_{\mathrm{sc}}\right)$ and $\operatorname{Conf}_{d}^{b}\left(\mathcal{A}_{\mathrm{ad}}\right)$. The difference between the two decorated versions is similar to the difference between double Bruhat cells associated to simply connected forms and adjoint forms. There is one more version of double Bott-Samelson cell $\operatorname{Conf}_{d}^{b}\left(\mathcal{A}_{\mathrm{sc}}^{\mathrm{fr}}\right)$, but it will not play a significant role in the present article.

We prove the following result on cluster structures of double Bott-Samelson cells.

Theorem 1.1 (Theorems 2.30, 3.45 and 3.46). The double Bott-Samelson cells $\operatorname{Conf}_{d}^{b}\left(\mathcal{A}_{\mathrm{sc}}\right)$ and $\operatorname{Conf}_{d}^{b}\left(\mathcal{A}_{\mathrm{ad}}\right)$ are smooth affine varieties. The coordinate ring $\mathcal{O}\left(\operatorname{Conf}_{d}^{b}\left(\mathcal{A}_{\mathrm{sc}}\right)\right)$ is an upper cluster algebra and $\mathcal{O}\left(\operatorname{Conf}_{d}^{b}\left(\mathcal{A}_{\mathrm{ad}}\right)\right)$ is a cluster Poisson algebra. ${ }^{I}$ The pair $\left(\operatorname{Conf}_{d}^{b}\left(\mathcal{A}_{\mathrm{sc}}\right), \operatorname{Conf}_{d}^{b}\left(\mathcal{A}_{\mathrm{ad}}\right)\right)$ forms a cluster ensemble.

Because double Bruhat cells are special cases of double Bott-Samelson cells, it follows from our result that the cluster structures on double Bruhat cells in the symmetrisable cases are canonical in the sense that they do not depend on the choice of reduced words (initial seeds), solving a conjecture of Berenstein, Fomin and Zelevinsky [BFZ05, Remark 2.14].

Inside a given upper cluster algebra, the subalgebra generated by all cluster variables is called its cluster algebra ${ }^{2}$ [FZ02, BFZ05]. An interesting question to ask is whether an upper cluster algebra coincides with its cluster algebra. Sufficient conditions to prove this equality include acyclicity [BFZ05], local acyclicity [Mul14] and CGL extensions [GY18]. In this article, we provide a new family of cluster varieties for which this equality holds.

Theorem 1.2 (Theorem 4.13). The upper cluster alegbra $\mathcal{O}\left(\operatorname{Conf}_{d}^{b}\left(\mathcal{A}_{\mathrm{sc}}\right)\right)$ coincides with its cluster algebra.

\subsection{Donaldson-Thomas Transformation and Periodicity Conjecture}

On every cluster variety there is a special formal automorphism called the Donaldson-Thomas transformation, which is closely related to the Donaldson-Thomas invariants of certain 3D Calabi-Yau category with stability conditions considered by Kontsevich and Soibelman [KS08]. Following the work of Gross et al. [GHKK18], if the Donaldson-Thomas transformation is a cluster transformation, then the FockGoncharov cluster duality conjecture holds. The cluster nature of Donaldson-Thomas transformations has been verified on many examples of cluster ensembles, including moduli spaces of G-local systems [GS18], Grassmannians [Wen21] and double Bruhat cells [Wen20]. As a direct consequence, the cluster duality conjecture holds in those cases.

\footnotetext{
${ }^{1}$ See Definition A.18.

${ }^{2}$ In this article, we always assume that frozen cluster variables are invertible.
} 
In the present article we explicitly realise the Donaldson-Thomas transformation of the double Bott-Samelson cell $\operatorname{Conf}_{d}^{b}(\mathcal{B})$ as a sequence of reflection maps followed by a transposition map (see Subsection 2.3 for their definitions). We prove the following statement.

Theorem 1.3 (Theorems 4.8, 4.10). The Donaldson-Thomas transformation of $\operatorname{Conf}_{d}^{b}(\mathcal{B})$ is a cluster transformation. The Fock-Goncharov duality conjecture ${ }^{3}$ holds for $\left(\operatorname{Conf}_{d}^{b}\left(\mathcal{A}_{\mathrm{sc}}\right), \operatorname{Conf}_{d}^{b}\left(\mathcal{A}_{\mathrm{ad}}\right)\right)$.

The key ingredients for constructing Donaldson-Thomas transformations are four reflection maps, ${ }^{i} r,{ }_{i} r, r^{i}$ and $r_{i}$, which are biregular isomorphisms between double Bott-Samelson cells that differ by the placement of $s_{i}$ :

$$
\operatorname{Conf}_{d}^{s_{i} b}(\mathcal{A}) \underset{{ }_{i} r}{\stackrel{{ }^{i} r}{\rightleftarrows}} \operatorname{Conf}_{s_{i} d}^{b}(\mathcal{A}) \quad \operatorname{Conf}_{d}^{b s_{i}}(\mathcal{A}) \underset{r_{i}}{\stackrel{r^{i}}{\rightleftarrows}} \operatorname{Conf}_{d s_{i}}^{b}(\mathcal{A})
$$

We prove the following result on these reflection maps.

Theorem 1.5 (Corollary 4.12). Reflection maps are quasi-cluster transformations and hence are Poisson maps.

In Section 5 we investigate the periodicity of Donaldson-Thomas transformations for a class of double Bott-Samelson cells associated to semisimple algebraic groups. We prove the following.

Theorem 1.6 (Theorem 5.1). If $\mathrm{G}$ is semisimple and the positive braids $(b, d)$ satisfy $\left(d b^{\circ}\right)^{m}=$ $w_{0}^{2 n}$, then the Donaldson-Thomas transformation of $\operatorname{Conf}_{d}^{b}(\mathcal{B})$ is of a finite order dividing $2(m+n)$.

Zamolodchikov's periodicity conjecture asserts that the solution of the $Y$-system associated to a pair of Dynkin diagrams is periodic with period relating to the Coxeter numbers of the two Dynkin diagrams. Keller gave a categorical proof of the conjecture in full generality in [Kel13].

Let $\Delta$ be a Dynkin diagram of finite type and let $\mathrm{G}$ be a group of type $\Delta$. In this article we relate the product $\Delta \square \mathrm{A}_{n}$ to a double Bott-Samelson cell associated to $\mathrm{G}$ and give a new geometric proof of Zamolodchikov's periodicity conjecture (Corollary 5.10).

As explained in [Kel11, Section 5.7], Zamolodchikov's periodicity implies a result on the periodicity of the Donaldson-Thomas transformation. Weng [Wen21] gave a direct geometric proof of the periodicity of DT in the case of $\mathrm{A}_{m} \square \mathrm{A}_{n}$ by realizing the Donaldson-Thomas transformation as a biregular automorphism on a configuration space of lines.

Theorem 1.6 gives a new geometric proof of the periodicity of DT in the cases of $\Delta \square \mathrm{A}_{n}$.

Theorem 1.7 (Corollary 5.11). Let $\Delta$ be a Dynkin quiver of finite type. Then $\mathrm{DT}_{\Delta \square \mathrm{A}_{n}}$ is of a finite order dividing $\frac{2(h+n+1)}{\operatorname{gcd}(h, n+1)}$ where $h$ is the Coxeter number of $\Delta$.

\subsection{Positive Braids Closures}

Let $(b, d)$ be a pair of positive braids in the braid group of type $\mathrm{A}_{r}$. Every word $(\mathbf{i}, \mathbf{j})$ of $(b, d)$ encodes two sequences of crossings at the top and at the bottom of a Legendrian link $\Lambda_{\mathbf{j}}^{\mathbf{i}}$ embedded in the standard contact $\mathbb{R}^{3}$ (see Subsection 6.2). Legendrian links obtained from different words of $(b, d)$ are related by Legendrian Reidemeister moves and therefore are Legendrian isotopic. Abusing notations we denote the corresponding isotopic class of Legendrian links by $\Lambda_{d}^{b}$. 
The reflection maps (1.4) correspond to Legendrian isotopies that move a crossing from top to bottom or vice versa at the two ends of the link diagram. The following picture depicts such a move for the reflection maps ${ }^{1} r \circ r^{2}: \operatorname{Conf}_{s_{1}}^{s_{1} s_{2}}(\mathcal{A}) \rightarrow \operatorname{Conf}_{s_{1} s_{1} s_{2}}^{e}(\mathcal{A})$ of Dynkin type $\mathrm{A}_{2}$.

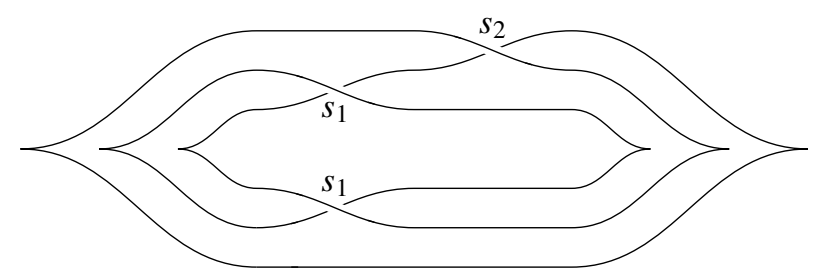

$$
\begin{gathered}
b=s_{1} s_{2} \\
d=s_{1}
\end{gathered}
$$
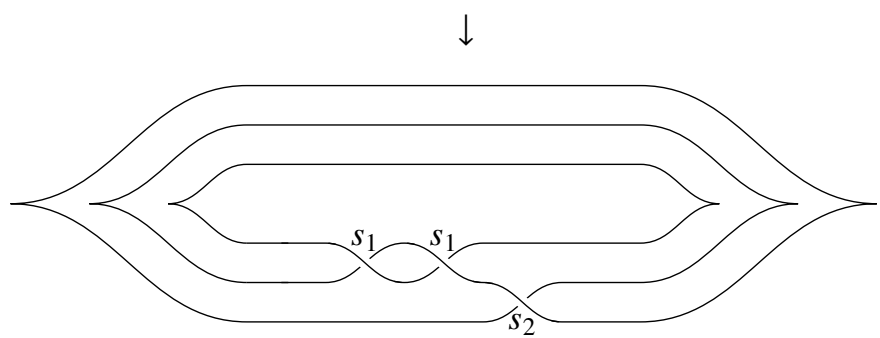

$$
b=e
$$$$
d=s_{1} s_{1} s_{2}
$$

Shende, Treumann and Zaslow STZ17] introduced a moduli space of microlocal rank-1 sheaves $\mathcal{M}_{1}(\Lambda)$ associated to any Legendrian link $\Lambda$. By a result of Guillermou, Kashiwara and Schapira [GKS12], the moduli spaces $\mathcal{M}_{1}(\Lambda)$ and $\mathcal{M}_{1}\left(\Lambda^{\prime}\right)$ are isomorphic if $\Lambda$ and $\Lambda^{\prime}$ are Legendrian isotopic [STZ17, Theorem 1.1]. However, one should keep in mind that the isomorphisms between such moduli spaces depends on the Legendrian isotopies.

By comparing the definitions of $\mathcal{M}_{1}\left(\Lambda_{\mathbf{j}}^{\mathbf{i}}\right)$ and $\operatorname{Conf}_{d}^{b}(\mathcal{B})$ we obtain the following result.

Theorem 1.8 (Theorem 6.14). There is a natural isomorphism $\mathcal{M}_{1}\left(\Lambda_{\mathbf{j}}^{\mathbf{i}}\right) \cong \operatorname{Conf}_{d}^{b}(\mathcal{B})$.

Theorem 1.8 implies that the automorphisms on the moduli spaces $\mathcal{M}_{1}\left(\Lambda_{d}^{b}\right)$ induced by braid moves are all trivial; therefore, one can canonically identify $\mathcal{M}_{1}\left(\Lambda_{\mathbf{j}}^{\mathbf{i}}\right)$ for different choices of words for $(b, d)$ and define the moduli space $\mathcal{M}_{1}\left(\Lambda_{d}^{b}\right)$ for a pair of positive braids $(b, d)$.

The cells $\operatorname{Conf}_{d}^{b}(\mathcal{A})$ associated to any generalised Cartan matrices are well defined over any finite field $\mathbb{F}_{q}$. Let

$$
f_{d}^{b}(q):=\left|\operatorname{Conf}_{d}^{b}(\mathcal{A})\left(\mathbb{F}_{q}\right)\right|
$$

In Subsection 6.1 we provide an algorithm for computing $f_{d}^{b}(q)$. The cell $\operatorname{Conf}_{d}^{b}(\mathcal{B})$ is isomorphic to $\operatorname{Conf}_{d}^{b}(\mathcal{A})$ modulo a $\mathrm{T} \times \mathrm{T}$ action. Let $r$ be the rank of the Cartan subgroup T. The orbifold counting of $\mathbb{F}_{q}$-points of $\operatorname{Conf}_{d}^{b}(\mathcal{B})$ is

$$
g_{d}^{b}(q):=\left|\operatorname{Conf}_{d}^{b}(\mathcal{B})\left(\mathbb{F}_{q}\right)\right|=\frac{\left|\operatorname{Conf}_{d}^{b}(\mathcal{A})\left(\mathbb{F}_{q}\right)\right|}{\left|\mathrm{T} \times \mathrm{T}\left(\mathbb{F}_{q}\right)\right|}=\frac{f_{d}^{b}(q)}{(q-1)^{2 r}} .
$$

In general, $g_{d}^{b}(q)$ is a rational function, with possible poles at $q=1$.

Theorem 1.9 (Corollary 6.15). Let $(b, d)$ be a pair of positive braids in the braid group of type $\mathrm{A}_{r}$. The double Bott-Samelson cell $\operatorname{Conf}_{d}^{b}(\mathcal{B})$ (as an algebraic stack) and the rational function $g_{d}^{b}(q)$ are Legendrian link invariants for the positive braid closure $\Lambda_{d}^{b}$. 


\subsection{Further Questions}

Comparison with Generalised Double Bruhat Cells. Let $\mathbf{u}=\left(u_{1}, u_{2}, \ldots, u_{n}\right)$ and $\mathbf{v}=\left(v_{1}, v_{2}, \ldots, v_{n}\right)$ be two $n$-tuples of Weyl group elements and let $C$ be a conjugacy class in G. Define

$$
\mathrm{B}_{+} \mathbf{u B}_{+}:=\left\{\left[x_{1}, \ldots, x_{n}\right] \in \underset{\mathrm{B}_{+}}{\mathrm{G}} \underset{\mathrm{B}_{+}}{\times} \underset{\mathrm{G}}{\times} \mid x_{i} \in \mathrm{B}_{+} u_{i} \mathrm{~B}_{+}\right\}
$$

and define $\mathrm{B}_{-} \mathbf{v B}_{-}$similarly. Lu and Mouquin [LM17] defined a generalised double Bruhat cell as

$$
\mathbf{G}_{C}^{\mathbf{u}, \mathbf{v}}:=\left\{\begin{array}{c|c}
{\left[x_{1}, \ldots, x_{n},\right.} & {\left[x_{i}\right] \in \mathrm{B}_{+} \mathbf{u B}_{+},\left[y_{i}\right] \in \mathrm{B}_{-} \mathbf{v B}_{-},} \\
\left.y_{1}, \ldots, y_{n}\right] & \left(x_{1} \ldots x_{n}\right)\left(y_{1} \ldots y_{n}\right)^{-1} \in C
\end{array}\right\} .
$$

Note that when $C=\{e\}$ and $n=1$, it coincides with the ordinary double Bruhat cells.

Let us lift $u_{i}$ and $v_{j}$ to positive braid elements and set $b=u_{1} \ldots u_{n}$ and $d=v_{1} \ldots v_{n}$. The generalised double Bruhat cell $\mathrm{G}_{\{e\}}^{\mathbf{u}, \mathbf{v}}$ is biregularly isomorphic to our decorated double Bott-Samelson cell $\operatorname{Conf}_{d}^{b}(\mathcal{A})$ via the following $\operatorname{map}^{4}$ :

$$
\begin{aligned}
\mathrm{G}_{\{e\}}^{\mathbf{u}, \mathbf{v}} & \longrightarrow \operatorname{Conf}_{d}^{b}(\mathcal{A}) \\
\left.y_{1}, \ldots, y_{n}\right] & \longmapsto\left[\begin{array}{l}
\mathrm{U}_{+}, \ldots, x_{n} \stackrel{u_{1}}{\longrightarrow} x_{1} \mathrm{~B}_{+} \stackrel{u_{2}}{\longrightarrow} x_{1} x_{2} \mathrm{~B}_{+} \stackrel{u_{3}}{\longrightarrow} \ldots \stackrel{u_{n}}{\longrightarrow} x_{1} \ldots x_{n} \mathrm{~B}_{+} \\
\mathrm{B}_{-} \underset{v_{1}}{\longrightarrow} y_{1} \mathrm{~B}_{-} \underset{v_{2}}{\longrightarrow} y_{1} y_{2} \mathrm{~B}_{-} \underset{v_{3}}{\longrightarrow} \ldots \stackrel{v_{n}}{\longrightarrow} y_{1} \ldots y_{n} \mathrm{U}_{-}
\end{array}\right]
\end{aligned}
$$

In particular, this isomorphism shows that the generalised double Bruhat cells $\mathrm{G}_{\{e\}}^{\mathbf{u}, \mathbf{v}}$ admit natural cluster structures. It further implies the following new result on generalised double Bruhat cells.

Corollary 1.10. Let $\mathbf{u}=\left(u_{1}, \ldots, u_{n}\right), \mathbf{v}=\left(v_{1}, \ldots, v_{n}\right), \mathbf{u}^{\prime}=\left(u_{1}^{\prime}, \ldots, u_{m}^{\prime}\right)$ and $\mathbf{v}^{\prime}=\left(v_{1}^{\prime}, \ldots, v_{m}^{\prime}\right)$. If $u_{1} \ldots u_{n}=u_{1}^{\prime} \ldots u_{m}^{\prime}$ and $v_{1} \ldots v_{n}=v_{1}^{\prime} \ldots v_{m}^{\prime}$ in the braid group, then there is a canonical isomorphism between $\mathrm{G}_{\{e\}}^{\mathbf{u}, \mathbf{v}}$ and $\mathrm{G}_{\{e\}}^{\mathbf{u}^{\prime}, \mathbf{v}^{\prime}}$.

Remark 1.11. We conjecture that the same statement holds for other conjugacy classes $C \neq\{e\}$. In [LM17], Lu and Mouquin defined a Poisson structure on $\mathrm{G}_{\{e\}}^{\mathbf{u}, \mathbf{v}}$ by pushing forward the Poisson structure on products of flag varieties. In the adjoint form cases $\mathrm{G}=\mathrm{G}_{\mathrm{ad}}$, the space $\operatorname{Conf}_{d}^{b}\left(\mathcal{A}_{\mathrm{ad}}\right)$ carries a natural Poisson structure from its cluster Poisson structure. We believe that these two Poisson structures coincide, but a detailed check is needed before we draw any definite conclusion.

In a recent work, Mouquin [Mou19] proved that the generalised double Bruhat cell $\mathrm{G}_{\{e\}}^{\mathbf{u}, \mathbf{u}}$ is a Poisson groupoid over the generalised Bruhat cell $\mathrm{G}_{\{e\}}^{\mathrm{e}, \mathrm{u}}$. We believe that the Poisson groupoid structure coincides with Fock-Goncharov's symplectic double for cluster varieties [FG09b]. We further observe that the inverse map of this Poisson groupoid resembles the Donaldson-Thomas transformation on Conf ${ }_{b}^{e}\left(\mathcal{A}_{\mathrm{ad}}\right)$, and we would like to see a further investigation in these directions.

In general, the decorated double Bott-Samelson cells do not cover the cases when $C \neq\{e\}$. Therefore, we post the following question.

Problem 1.12. Is there a way to generalise the decorated double Bott-Samelson construction to include all generalised double Bruhat cells? If yes, how do the Poisson structures arising from the two approaches compare to each other?

We expect that such a generalisation (if it exists) is related to the braid cell defined below.

${ }^{4}$ The isomorphism was pointed out to us by J.H. Lu. 
Braid Cell. Let $G$ be a split semisimple algebraic group. The general position condition between Borel subgroups $B \longrightarrow B^{\prime}$ can be rewritten as $B \stackrel{w_{0}}{\longrightarrow} B^{\prime}$ (see Notation 2.3). In this case, a double Bott-Samelson cell can be defined as a configuration space of Borel subgroups satisfying the following relative position relation:

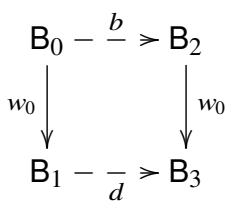

where the top and bottom dashed arrows represent a chain of flags with relative position conditions imposed by the positive braids $b$ and $d$, respectively. When the words of $b$ and $d$ are reduced, the double Bott-Samelson cell $\operatorname{Conf}_{v}^{u}(\mathcal{A})$ is naturally isomorphic to the double Bruhat cells $\mathrm{G}^{u, v}$.

In [WY07], Webster and Yakimov introduced a variety $\mathcal{P}_{v, w}^{u}$ associated to a triple of Weyl group elements $(u, v, w)$, which can be defined as the configuration space of Borel subgroups satisfying the following relative position relation:

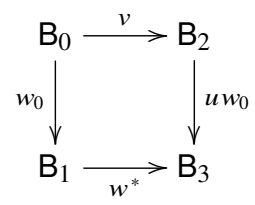

where $w^{*}:=w_{0} w w_{0}^{-1}$ in the Weyl group. Because $\mathrm{B}_{1} \stackrel{w^{*}}{\longrightarrow} \mathrm{B}_{3}$ is equivalent to $\mathrm{B}_{1} \stackrel{w^{*-1}}{\longleftarrow} \mathrm{B}_{3}$, the above relative position relation diagram is equivalent to the following one:

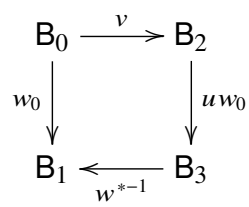

The chain $\mathrm{B}_{0} \stackrel{v}{\longrightarrow} \mathrm{B}_{2} \stackrel{u w_{0}}{\longrightarrow} \mathrm{B}_{3} \stackrel{w^{*-1}}{\longrightarrow} \mathrm{B}_{1}$ can be treated as a chain of Borel subgroups with relative position condition imposed by a braid $b$, where $b$ is the concatenation of any triple of reduced words of $v, u w_{0}$ and $w^{*-1}$. The above relative position relation diagram reduces to the following one:

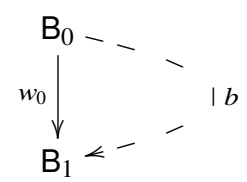

Let us take one step further by allowing $b$ to be any positive braid. The moduli space parametrising the configuration (1.13) is called a braid cell $\operatorname{Conf}_{b}(\mathcal{B})$. By putting decorations on $\mathrm{B}_{0}$ and $\mathrm{B}_{1}$ we can define its decorated version $\operatorname{Conf}_{b}(\mathcal{A})$. The cells $\operatorname{Conf}_{b}(\mathcal{A})$ generalise the open Richardson varieties [LS16]. Following the proof of Theorem 1.1, one can show that $\operatorname{Conf}_{b}(\mathcal{A})$ is an affine variety. We make the following conjecture.

Conjecture 1.14. The coordinate ring of $\operatorname{Conf}_{b}\left(\mathcal{A}_{s c}\right)$ is an upper cluster algebra.

Legendrian Link Invariants. As stated earlier, the double Bott-Samelson cell $\operatorname{Conf}_{d}^{b}(\mathcal{B})$ associated to Dynkin type $\mathrm{A}_{r}$ is a Legendrian link invariant for positive braids closures. Furthermore, Legendrian 
isotopies between the positive braid closures $\Lambda_{d}^{b}$ and $\Lambda_{d^{\prime}}^{b^{\prime}}$ give rise to isomorphisms between $\operatorname{Conf}_{d}^{b}(\mathcal{B})$ and $\operatorname{Conf}_{d^{\prime}}^{b^{\prime}}(\mathcal{B})$. We propose the following conjecture.

Conjecture 1.15. The isomorphisms $\operatorname{Conf}_{d}^{b}(\mathcal{B}) \stackrel{\cong}{\longrightarrow} \operatorname{Conf}_{d^{\prime}}^{b^{\prime}}(\mathcal{B})$ associated to Legendrian isotopies between $\Lambda_{d}^{b}$ and $\Lambda_{d^{\prime}}^{b^{\prime}}$ are cluster Poisson transformations.

One strategy to prove this conjecture is to equip $\mathcal{M}_{1}(\Lambda)$ with a cluster Poisson structure for any Legendrian link $\Lambda$ and then show that the Legendrian versions of the Reidemeister moves induce isomorphisms that preserve the cluster Poisson structures. Note that this is already true for the third Legendrian Reidemeister moves - that is, the braid moves - but defining a cluster Poisson structure on $\mathcal{M}_{1}(\Lambda)$ and showing the cluster-ness of the remaining two Legendrian Reidemeister moves still seem to be quite difficult tasks.

Theorem 1.8 naturally induces a cluster Poisson structure on $\mathcal{M}_{1}\left(\Lambda_{d}^{b}\right)$. In [STWZ19], Shende et al. studied cluster Poisson structures on $\mathcal{M}_{1}(\Lambda)$ for Lengendrian links $\Lambda \subset T^{\infty} \mathbb{R}^{2}$ that come from conormal lifts of immersed curves in $\mathbb{R}^{2}$. Although their ambient contact manifold $T^{\infty} \mathbb{R}^{2}$ is different from ours (which is the standard contact $\mathbb{R}^{3}$ ), it is still worthwhile to compare these two setups and the resulting cluster Poisson structures. Therefore, we pose the following question.

Problem 1.16. How much does $\mathcal{M}_{1}(\Lambda)$ depend on the ambient contact manifold of $\Lambda$ ? Do the cluster Poisson structures obtained from double Bott-Samelson cells coincide with those in [STWZ19] for Legendrian links that can be embedded in both ways?

Shende, Treumann and Zaslow [STZ17] introduced a category $\mathbf{S h}_{\Lambda}^{\bullet}\left(\mathbb{R}^{2}\right)$ of constructible sheaves with singular support controlled by $\Lambda$, which can be viewed as a 'categorification' of $\operatorname{Conf}_{d}^{b}(\mathcal{B}) \cong$ $\mathcal{M}_{1}\left(\Lambda_{d}^{b}\right)$. Conjecture 1.15 implies that the cluster $\mathrm{K}_{2}$ counterpart of $\operatorname{Conf}_{d}^{b}(\mathcal{B})$, namely, $\operatorname{Conf}_{d}^{b}\left(\mathcal{A}_{\mathrm{sc}}^{\mathrm{fr}}\right)$, is a Legendrian link invariant as well. We further ask the following.

Problem 1.17. Is there a categorification of $\operatorname{Conf}_{d}^{b}\left(\mathcal{A}_{\mathrm{sc}}^{\mathrm{fr}}\right)$ associated to $\Lambda_{d}^{b}$ ?

As observed from the examples, we conjecture that the number of components in $\Lambda_{d}^{b}$ is equal to $1-\operatorname{ord}_{q=1} g_{d}^{b}(q)$. In particular, $g_{d}^{b}(q)$ is a polynomial when $\Lambda_{d}^{b}$ is a knot.

\section{Double Bott-Samelson Cells}

\subsection{Flags, Decorated Flags, Relative Position and Compatibility}

In this section, we fix notations and investigate several elementary properties of flag varieties.

Let $\mathrm{C}$ be an $r \times r$ symmetrisable generalised Cartan matrix of corank $l$. Let $\tilde{r}:=r+l$.

Let $\left(\mathrm{G}, \mathrm{B}_{+}, \mathrm{B}_{-}, \mathrm{N}, \mathrm{S}\right)$ be a twin Tits system ${ }^{5}$ associated to $\mathrm{C}$. Here $\mathrm{G}$ is a Kac-Peterson group, $\mathrm{B}_{+}$and $B_{-}$are opposite Borel subgroups of $G, N$ is the normaliser of $T:=B_{+} \cap B_{-}$in $G$ and $S$ is a set of Coxeter generators for the Weyl group $\mathrm{W}:=\mathrm{N} / \mathrm{T}$.

Let $\varepsilon \in\{+,-\}$. We define two flag varieties:

$$
\mathcal{B}_{\varepsilon}:=\left\{\text { Borel subgroups of } G \text { that are conjugate to } B_{\varepsilon}\right\} .
$$

The group $G$ acts transitively on $\mathcal{B}_{\varepsilon}$ by conjugation, with $B_{\varepsilon}$ self-stabilizing. Therefore, we obtain natural isomorphisms

$$
\mathcal{B}_{\varepsilon} \cong \mathrm{G} / \mathrm{B}_{\varepsilon} \cong \mathrm{B}_{\varepsilon} \backslash \mathrm{G}
$$

${ }^{5}$ We include a summary of twin Tits system in the appendix; see [Abr96] and [Kum02] for more details. 
that identify Borel subgroups conjugate to $B_{\varepsilon}$ with left and right cosets of $B_{\varepsilon}$. When switching left and right cosets, we get $x \mathrm{~B}_{\varepsilon}=\mathrm{B}_{\varepsilon} x^{-1}$. Abusing notation, we shall use the terms 'Borel subgroups' and 'flags' interchangeably throughout this article.

Let $\mathrm{U}_{\varepsilon}=\left[\mathrm{B}_{\varepsilon}, \mathrm{B}_{\varepsilon}\right]$ be the maximal unipotent subgroups inside $\mathrm{B}_{\varepsilon}$. Define decorated flag varieties

$$
\mathcal{A}_{+}:=\mathrm{G} / \mathrm{U}_{+} \text {and } \mathcal{A}_{-}:=\mathrm{U}_{-} \backslash \mathrm{G} \text {. }
$$

The inclusions $\mathrm{U}_{\varepsilon} \hookrightarrow \mathrm{B}_{\varepsilon}$ give rise to natural projections

$$
\mathcal{A}_{+}=\mathrm{G} / \mathrm{U}_{+} \rightarrow \mathrm{G} / \mathrm{B}_{+} \cong \mathcal{B}_{+} \text {and } \mathcal{A}_{-}=\mathrm{U}_{-} \backslash \mathrm{G} \rightarrow \mathrm{B}_{-} \backslash \mathrm{G} \cong \mathcal{B}_{-} .
$$

We say that $\mathrm{A} \in \mathcal{A}_{\varepsilon}$ is a decorated flag over $\mathrm{B} \in \mathcal{B}_{\varepsilon}$ if $\mathrm{B}$ is the image of $\mathrm{A}$ under the above projections.

All $\mathrm{G}$-actions in this article are left actions unless otherwise specified. For example, $g \in \mathrm{G}$ acts on $\mathcal{A}_{-}$by $g .\left(\mathrm{U}_{-} x\right):=\mathrm{U}_{-} x g^{-1}$.

The transposition is an anti-involution of $\mathrm{G}$ that swaps $\mathrm{B}_{+}$and $\mathrm{B}_{-}$. It induces biregular isomorphisms between (decorated) flag varieties:

$$
\mathcal{B}_{+} \stackrel{t}{\longleftrightarrow} \mathcal{B}_{-} \text {and } \mathcal{A}_{+} \stackrel{t}{\longleftrightarrow} \mathcal{A}_{-}
$$

The images of $B$ and $A$ under transposition are denoted by $B^{t}$ and $A^{t}$, respectively.

Notation 2.1. We use superscripts for elements in $\mathcal{B}_{+}$, subscripts for elements in $\mathcal{B}_{-}$and parenthesis notations for elements in either flag variety; for example,

(1) Elements of $\mathcal{B}_{+}: \mathrm{B}^{0}, \mathrm{~B}^{1}, \mathrm{~B}^{2}, \ldots$

(2) Elements of $\mathcal{B}_{-}: B_{0}, B_{1}, B_{2}, \ldots$

(3) Elements that are in either $\mathcal{B}_{+}$or $\mathcal{B}_{-}: \mathrm{B}, \mathrm{B}(0), \mathrm{B}(1), \mathrm{B}(2), \ldots$

The same rule applies to decorated flags.

In this article we focus on a pair of Kac-Peterson groups $G_{s c}$ and $G_{a d}$. For semisimple cases, $G_{s c}$ and $\mathrm{G}_{\mathrm{ad}}$ are the simply connected and adjoint semisimple algebraic groups, respectively. In general, when the Cartan matrix $C$ is not invertible, the construction of $\mathrm{G}_{\mathrm{sc}}$ and $\mathrm{G}_{\mathrm{ad}}$ depends on the choices of a lattice $P \subset \mathfrak{h}^{*}$ and a basis $\left\{\omega_{i}\right\}_{i=1}^{\tilde{r}}$ of P. See Appendix A for details.

The center of $G_{s c}$ contains a finite subgroup $Z$ such that $G_{a d} \cong G_{s c} / Z$. Note that $Z \subset B_{\varepsilon}$. Therefore, the flag varieties $\mathcal{B}_{\varepsilon}$ associated to either group are isomorphic. For the decorated flag varieties, the covering map $\mathrm{G}_{\mathrm{sc}} \rightarrow \mathrm{G}_{\mathrm{ad}}$ induces a $|\mathrm{Z}|$-to-1 covering map $\pi: \mathcal{A}_{\mathrm{sc}, \varepsilon} \rightarrow \mathcal{A}_{\mathrm{ad}, \varepsilon}$, respectively.

Let $\mathrm{G}$ be either $\mathrm{G}_{\mathrm{sc}}$ or $\mathrm{G}_{\mathrm{ad}}$. The group $\mathrm{G}$ admits Bruhat decompositions

$$
\mathrm{G}=\bigsqcup_{w \in \mathrm{W}} \mathrm{B}_{+} w \mathrm{~B}_{+}=\bigsqcup_{w \in \mathrm{W}} \mathrm{B}_{-} w \mathrm{~B}_{-}
$$

and a Birkhoff decomposition

$$
\mathrm{G}=\bigsqcup_{w \in \mathrm{W}} \mathrm{B}_{-} w \mathrm{~B}_{+} .
$$

Every $x \in \mathrm{B}_{-} \mathrm{B}_{+}=\mathrm{U}_{-} \mathrm{TU}_{+}$admits a unique decomposition (a.k.a. the Gaussian decomposition)

$$
x=[x]_{-}[x]_{0}[x]_{+}
$$

with $[x]_{\varepsilon} \in \cup_{\varepsilon}$ and $[x]_{0} \in \mathrm{T}$. Such an element $x$ is called Gaussian decomposable.

The above decompositions induce two W-valued 'distance' functions and a W-valued 'codistance' function that are invariant under G-diagonal actions. 
Definition 2.2. A pair of flags $\left(x \mathrm{~B}_{\varepsilon}, y \mathrm{~B}_{\varepsilon}\right)$ is of Tits distance $d_{\varepsilon}\left(x \mathrm{~B}_{\varepsilon}, y \mathrm{~B}_{\varepsilon}\right)=w$ if $x^{-1} y \in \mathrm{B}_{\varepsilon} w \mathrm{~B}_{\varepsilon}$. A pair $\left(x \mathrm{~B}_{-}, y \mathrm{~B}_{+}\right)$is of Tits codistance $d\left(x \mathrm{~B}_{-}, y \mathrm{~B}_{+}\right)=w$ if $x^{-1} y \in \mathrm{B}_{-} w \mathrm{~B}_{+}$.

A pair $\left(\mathrm{B}_{0}, \mathrm{~B}^{0}\right)$ is said to be in general position (or opposite to each other) if $d\left(\mathrm{~B}_{0}, \mathrm{~B}^{0}\right)=e$.

Notation 2.3. We shall use the following notations to encode the Tits (co)distances between flags:

(1) $\mathrm{B}^{0} \stackrel{w}{\longrightarrow} \mathrm{B}^{1}$ means $d_{+}\left(\mathrm{B}^{0}, \mathrm{~B}^{1}\right)=w$.

(2) $\mathrm{B}_{0} \stackrel{w}{\longrightarrow} \mathrm{B}_{1}$ means $d_{-}\left(\mathrm{B}_{0}, \mathrm{~B}_{1}\right)=w$.

(3) $\mathrm{B}_{0} \stackrel{w}{-\mathrm{B}^{0}}$ means $d\left(\mathrm{~B}_{0}, \mathrm{~B}^{0}\right)=w$.

We often omit $w$ in the diagrams if $w=e$. Similar diagrams with decorated flags placed at one or both ends imply that the pair of underlying flags is of the indicated Tits (co)distance.

\section{Lemma 2.4.}

(1) $\mathrm{B} \stackrel{w}{\longrightarrow} \mathrm{B}^{\prime}$ if and only if $\mathrm{B}^{\prime t} \stackrel{w^{-1}}{\longrightarrow} \mathrm{B}^{t}$.

(2) $\mathrm{B} \stackrel{w}{-} \mathrm{B}^{\prime}$ if and only if $\mathrm{B}^{\prime t} \stackrel{w^{-1}}{-\mathrm{B}^{t}}$.

Proof. Obvious from the definition.

The following lemma will be used many times. Its proof is included in the Appendix.

Lemma 2.5. Let $u, v, w$ be Weyl group elements such that $u v=w$ and $l(u)+l(v)=l(w)$. In each of the following triangles, the black relative position holds if and only if the blue relative position holds. Furthermore, each blue flag is uniquely determined by the pair of black flags.
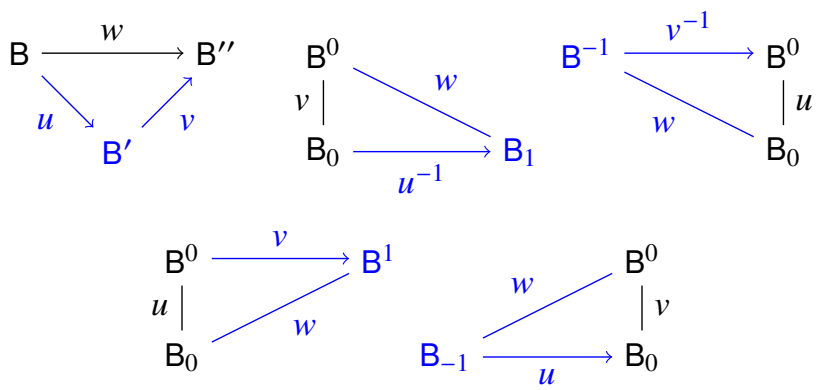

Every dominant weight $\lambda$ of $\mathrm{G}$ gives rise to a regular function $\Delta_{\lambda}$ on $\mathrm{G}$ such that $\Delta_{\lambda}(x)=\lambda\left([x]_{0}\right)$ for every $x \in U_{-} \mathrm{TU}_{+}$. They induce G-invariant functions

$$
\begin{aligned}
\Delta_{\lambda}: \mathcal{A}_{-} \times \mathcal{A}_{+} & \rightarrow \mathbb{A}^{1} \\
\left(\mathrm{U}_{-} x, y \mathrm{U}_{+}\right) & \mapsto \Delta_{\lambda}(x y) .
\end{aligned}
$$

When $\mathrm{G}=\mathrm{G}_{\mathrm{sc}}$, we take the fundamental weights $\omega_{1}, \ldots, \omega_{\tilde{r}}$ and set $\Delta_{i}:=\Delta_{\omega_{i}}$.

The following result is an easy consequence of the fact that $\Delta_{\lambda}$ is invariant under transposition.

Lemma 2.6. $\Delta_{\lambda}\left(A_{0}, A^{0}\right)=\Delta_{\lambda}\left(\left(A^{0}\right)^{t},\left(A_{0}\right)^{t}\right)$.

A result of Geiss, Leclerc and Schröer (Theorem A.11) allows us to detect that general position of decorated flags based on the $\Delta$ functions.

Theorem 2.7. A pair $\left(A_{0}, A^{0}\right)$ is in general position if and only if $\Delta_{\lambda}\left(A_{0}, A^{0}\right) \neq 0$ for every dominant $\lambda$.

Remark 2.8. It suffices to check the nonvanishing of a finite set of $\Delta_{\lambda}$. For example, when $\mathrm{G}=\mathrm{G}_{\mathrm{sc}}$, it suffices to check $\Delta_{i} \neq 0$ for all $i$. 
Every $w \in \mathrm{W}$ admit two special lifts to $\mathrm{G}$ denoted as $\bar{w}$ and $\overline{\bar{w}}$. The following are refined versions of Bruhat and Birkhoff decomposition:

$$
\mathrm{G}=\bigsqcup_{w \in \mathrm{W}} \mathrm{U}_{+} \mathrm{T} \bar{w} \mathrm{U}_{+}=\bigsqcup_{w \in \mathrm{W}} \mathrm{U}_{-} \overline{\bar{w}} \mathrm{TU} \mathrm{U}_{-}=\bigsqcup_{w \in \mathrm{W}} \mathrm{U}_{-} \mathrm{T} \bar{w} \mathrm{U}_{+} .
$$

The factor $t \in \mathrm{T}$ is uniquely determined for every $g \in \mathrm{G}$ in the above decompositions.

\section{Definition 2.9.}

(1) A pair of decorated flags $x \mathrm{U}_{+} \stackrel{w}{\longrightarrow} y \mathrm{U}_{+}$is compatible if $x^{-1} y \in \mathrm{U}_{+} \bar{w} \mathrm{U}_{+}$.

(2) A pair of decorated flags $U_{-} x \stackrel{w}{\longrightarrow} U_{-} y$ is compatible if $x y^{-1} \in U_{-} \overline{\bar{w}} U_{-}$.

Lemma 2.10. For $\mathrm{B} \stackrel{w}{\longrightarrow} \mathrm{B}^{\prime}$, a decoration on $\mathrm{B}$ uniquely determines a compatible decoration on $\mathrm{B}^{\prime}$ and vice versa.

Proof. It follows from the uniqueness of the T-factor in the refined version of Bruhat decompositions and Birkhoff decomposition.

The following lemma is an analogy of the first case of Lemma 2.5 for decorated flags.

Lemma 2.11. Suppose that $u v=w$ and $l(u)+l(v)=l(w)$.

(1) If $\mathrm{A} \stackrel{u}{\longrightarrow} \mathrm{A}^{\prime}$ and $\mathrm{A}^{\prime} \stackrel{v}{\longrightarrow} \mathrm{A}^{\prime \prime}$ are compatible, then so is $\mathrm{A} \stackrel{w}{\longrightarrow} \mathrm{A}^{\prime \prime}$.

(2) If $\mathrm{A} \stackrel{w}{\longrightarrow} \mathrm{A}^{\prime \prime}$ is compatible, then there is a unique $\mathrm{A}^{\prime}$ such that $\mathrm{A} \stackrel{u}{\longrightarrow} \mathrm{A}^{\prime}$ and $\mathrm{A}^{\prime} \stackrel{v}{\longrightarrow} \mathrm{A}^{\prime \prime}$ are compatible.

Proof. It follows from the fact that $\bar{w}=\bar{u} \bar{v}$ and $\overline{\bar{w}}=\overline{\bar{u}} \overline{\bar{v}}$.

Definition 2.12. A pinning is a pair of decorated flags $\left(\mathrm{U}_{-} x, y \mathrm{U}_{+}\right)$such that $x y \in \mathrm{U}_{-} \mathrm{U}_{+}$.

Lemma 2.13. The following conditions are equivalent:

(1) The pair $\left(\mathrm{A}_{0}, \mathrm{~A}^{0}\right)$ is a pinning.

(2) There exists a unique $z \in G$ such that $\left(\mathrm{A}_{0}, \mathrm{~A}^{0}\right)=\left(\mathrm{U}_{-} z^{-1}, z \mathrm{U}_{+}\right)$.

(3) we have $\Delta_{\lambda}\left(A_{0}, A^{0}\right)=1$ for every dominant weight $\lambda$.

Moreover, condition (2) implies that the action of $\mathrm{G}$ on the space of pinnings is free and transitive. When $\mathrm{G}=\mathrm{G}_{\mathrm{sc}}$, condition (3) can be replaced by showing that $\Delta_{\omega_{i}}\left(\mathrm{~A}_{0}, \mathrm{~A}^{0}\right)=1$ for $1 \leq i \leq \tilde{r}$.

Proof. $(1) \Longrightarrow(2)$. Suppose that $\left(\mathrm{A}_{0}, \mathrm{~A}^{0}\right)=\left(\mathrm{U}_{-} x, y \mathrm{U}_{+}\right)$is a pinning. Then $x y=[x y]_{-}[x y]_{+} \in \mathrm{U}^{-} \mathrm{U}^{+}$. Let $z:=x^{-1}[x y]_{-}=y[x y]_{+}^{-1}$. Then $\cup_{-} z^{-1}=\cup_{-}[x y]_{-}^{-1} x=\cup_{-} x$ and $z \cup_{+}=y[x y]_{+}^{-1} \cup_{+}=y \cup_{+}$. Note that $U_{-} z^{-1}=U_{-} z^{\prime-1}$ implies that $z^{-1} z^{\prime} \in U_{-}$and $z U_{+}=z^{\prime} U_{+}$implies that $z^{-1} z^{\prime} \in U_{+}$. Therefore, $z^{-1} z^{\prime} \in U_{-} \cap U_{+}=\{e\}$ and $z=z^{\prime}$. The uniqueness of $z$ follows.

(2) $\Longrightarrow$ (3). This is obvious from the definition of $\Delta_{\lambda}$.

(3) $\Longrightarrow(1)$. By Theorem 2.7, $\Delta_{\lambda}\left(A_{0}, A^{0}\right)=1$ implies that $\left.A_{0}, A^{0}\right)$ is in general opposition. Let $\mathrm{A}_{0}=U_{-} x$ and $A^{0}=y U_{+}$. The product $x y$ is Gaussian decomposable; that is, $x y=[x y]_{-}[x y]_{0}[x y]_{+}$. The condition $1=\Delta_{\lambda}\left(\mathrm{A}_{0}, \mathrm{~A}^{0}\right)=\Delta_{\lambda}\left([x y]_{0}\right)$ implies that $[x y]_{0}=e$. Therefore, $x y \in \mathrm{U}_{-} \mathrm{U}_{+}$.

Corollary 2.14. There is a one-to-one correspondence between pinnings and opposite pairs of flags in $\mathcal{A}_{-} \times \mathcal{B}_{+}$(respectively $\mathcal{B}_{-} \times \mathcal{A}_{+}$) given by the forgetful map.

Proof. Lemma 2.13 asserts that every pinning is of the form $\left(\mathrm{U}_{-} z^{-1}, z \mathrm{U}_{+}\right)$. Therefore, the forgetful map is surjective. For injectivity, if $\left(\mathrm{U}_{-} z^{-1}, z \mathrm{~B}_{+}\right)=\left(\mathrm{U}_{-} z^{\prime-1}, z^{\prime} \mathrm{B}_{+}\right)$in $\mathcal{A}_{-} \times \mathcal{B}_{+}$, then $z^{-1} z^{\prime} \in \mathrm{U}_{-} \cap \mathrm{B}_{+}=\{e\}$ and hence $z=z^{\prime}$. A similar proof can be applied to the $\mathcal{B}_{-} \times \mathcal{A}_{+}$cases.

The next lemma shows that notions of compatibility and pinnings respect the transposition. 


\section{Lemma 2.15.}

(1) $\mathrm{A} \stackrel{w}{\longrightarrow} \mathrm{A}^{\prime}$ is compatible if and only if $\mathrm{A}^{\prime t} \stackrel{w^{-1}}{\longrightarrow} \mathrm{A}^{t}$ is compatible.

(2) $\mathrm{A} \longrightarrow \mathrm{A}^{\prime}$ is a pinning if and only if $\mathrm{A}^{\prime t} \longrightarrow \mathrm{A}^{t}$ is a pinning.

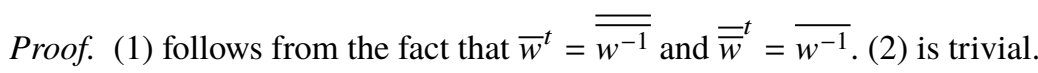

\subsection{Double Bott-Samelson Cells}

The semigroup $\mathrm{Br}_{+}$of positive braids is generated by symbols $s_{i}$ subject to the braid relations

$$
\underbrace{s_{i} s_{j} \ldots}_{m_{i j}}=\underbrace{s_{j} s_{i} \ldots,}_{m_{i j}},
$$

where $m_{i j}=2,3,4,6$ or $\infty$ according to whether $\mathrm{C}_{i j} \mathrm{C}_{j i}$ is $0,1,2,3$ or $\geq 4$.

A word for a positive braid $b \in \mathrm{Br}_{+}$is a sequence $\mathbf{i}=\left(i_{1}, i_{2}, \ldots, i_{n}\right)$ such that $b=s_{i_{1}} s_{i_{2}} \ldots s_{i_{n}}$. Denote by $\mathbf{H}(b)$ the set of all words for $b$.

For an arbitrary Weyl group element, its reduced words are related by braid relations. Hence, there is a set-theoretic lift $\mathrm{W} \hookrightarrow \mathrm{Br}_{+}$.

Definition 2.17. Let $\mathbf{i}=\left(i_{1}, \ldots, i_{n}\right) \in \mathbf{H}(b)$. An $\mathbf{i}$-chain of flags is a sequence of flags:

$$
\mathrm{B}(\mathbf{i}):=\mathrm{B}(0) \stackrel{s_{i_{1}}}{\longrightarrow} \mathrm{B}(1) \stackrel{s_{i_{2}}}{\longrightarrow} \cdots \stackrel{s_{i_{n}}}{\longrightarrow} \mathrm{B}(n) .
$$

Denote by $\mathcal{C}(\mathbf{i})$ the set of $\mathbf{i}$-chains of flags.

Lemma 2.5 allows us to do local changes to a chain of flags with prescribed relative positions. If $w=u_{1} u_{2}=v_{1} v_{2}$ in the Weyl group and $l(w)=l\left(u_{1}\right)+l\left(u_{2}\right)=l\left(v_{1}\right)+$ $l\left(v_{2}\right)$, then for every chain $\mathrm{B}(0) \stackrel{u_{1}}{\longrightarrow} \mathrm{B}(1) \stackrel{u_{2}}{\longrightarrow} \mathrm{B}(2)$, there is a unique flag $\mathrm{B}(3)$ such that $\mathrm{B}(0) \stackrel{v_{1}}{\longrightarrow} \mathrm{B}(3) \stackrel{v_{2}}{\longrightarrow} \mathrm{B}(2)$. Braid moves are a class of special local changes: whenever the braid relation (2.16) holds, one can change a chain of flags uniquely from

$$
\ldots \mathrm{B}(k) \stackrel{s_{i}}{\rightarrow} \mathrm{B}(k+1) \stackrel{s_{j}}{\rightarrow} \mathrm{B}(k+2) \stackrel{s_{i}}{\longrightarrow} \ldots \mathrm{B}\left(k+m_{i j}-1\right) \stackrel{s_{i} \text { or } s_{j}}{\longrightarrow} \mathrm{B}\left(k+m_{i j}\right) \ldots
$$

to

$$
\ldots \mathrm{B}(k) \stackrel{s_{j}}{\rightarrow} \mathrm{B}^{\prime}(k+1) \stackrel{s_{i}}{\rightarrow} \mathrm{B}^{\prime}(k+2) \stackrel{s_{j}}{\rightarrow} \ldots \mathrm{B}^{\prime}\left(k+m_{i j}-1\right) \stackrel{s_{j} \text { or } s_{i}}{\longrightarrow} \mathrm{B}\left(k+m_{i j}\right) \ldots
$$

Let $\mathbf{i}, \mathbf{j} \in \mathbf{H}(b)$. Let $\tau$ be a sequence of braid moves taking $\mathbf{i}$ to $\mathbf{j}$. It further induces a bijection

$$
\tau_{\mathbf{i}}^{\mathbf{j}}: \mathcal{C}(\mathbf{i}) \longrightarrow \mathcal{C}(\mathbf{j})
$$

Theorem 2.18. If $\boldsymbol{i}=\boldsymbol{j}$, then $\tau_{\boldsymbol{i}}^{j}$ is the identity map.

Proof. Let $\mathbf{i}=\left(i_{1}, \ldots, i_{n}\right)$ and $\mathbf{j}=\left(j_{1}, \ldots, j_{n}\right)$. The map $\tau_{\mathbf{i}}^{\mathbf{j}}$ takes an $\mathbf{i}$-chain $\mathrm{B}(\mathbf{i})=(\mathrm{B}(0), \ldots, \mathrm{B}(n))$ to a $\mathbf{j}$-chain $\mathrm{B}(\mathbf{j})=\left(\mathrm{B}^{\prime}(0), \ldots, \mathrm{B}^{\prime}(n)\right)$. It suffices to prove that if $i_{k}=j_{k}$ for all $m<k \leq n$ then $\mathrm{B}^{\prime}(m)=\mathrm{B}(m)$.

Without loss of generality, we assume that $\mathrm{B}(i)=\mathrm{B}^{i} \in \mathcal{B}_{+}$. Let $\mathrm{B}_{0}$ be a flag opposite to $\mathrm{B}^{0}$. By the second case of Lemma 2.5 for $(u, v, w)=\left(s_{i}, e, s_{i}\right)$, we get a unique $\mathrm{B}_{1}$ such that

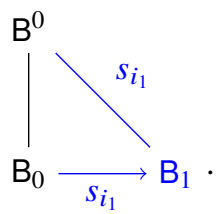


The third case of Lemma 2.5 implies that $\left(B_{1}, B^{1}\right)$ is in general position. Repeating the same construction through the whole sequence, we obtain an $\mathbf{i}$-chain $\mathrm{B}(\mathbf{i})^{o p}=\left(\mathrm{B}_{0}, \mathrm{~B}_{1}, \ldots, \mathrm{B}_{n}\right)$, called an opposite chain of $\mathrm{B}(\mathbf{i})$, such that $\left(\mathrm{B}_{k}, \mathrm{~B}^{k}\right)$ is in general position for $0 \leq k \leq n$. The chain $\mathrm{B}(\mathbf{i})^{\text {op }}$ is uniquely determined by the choice of $\mathrm{B}_{0}$.

Let us apply a braid move $\tau$ to $\mathrm{B}(\mathbf{i})$, obtaining a $\tau(\mathbf{i})$-chain $\mathrm{B}(\tau(\mathbf{i}))$. Applying the same braid move $\tau$ to $\mathrm{B}(\mathbf{i})^{o p}$, we claim that the obtained chain is an opposite chain of $\mathrm{B}(\tau(\mathbf{i}))$. We prove the claim for an $A_{2}$-type braid move below. The same argument works for $B_{2}-$ and $G_{2}$-type braid moves.

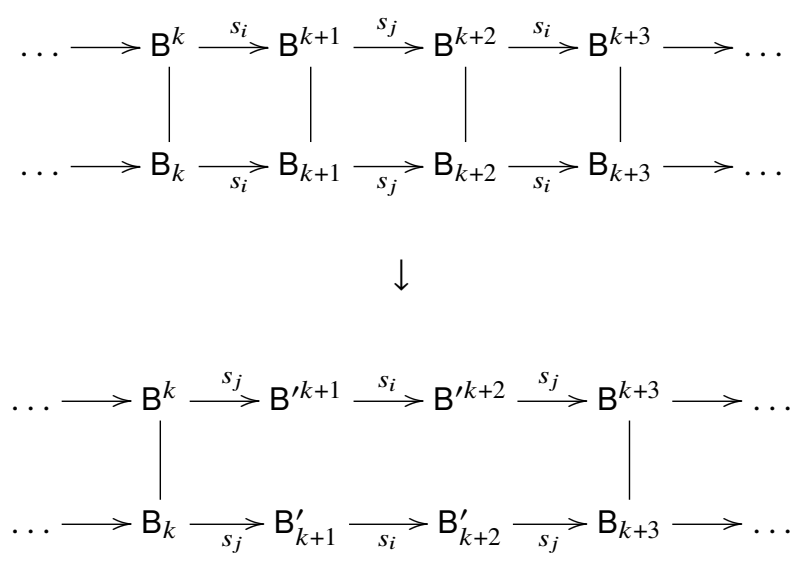

Note that a priori it is not obvious that the two pairs of flags in the middle are opposite. On the other hand, we may run the opposite chain construction with the new top chain starting with the same choice of $\mathrm{B}_{0}$, obtaining the following chain:

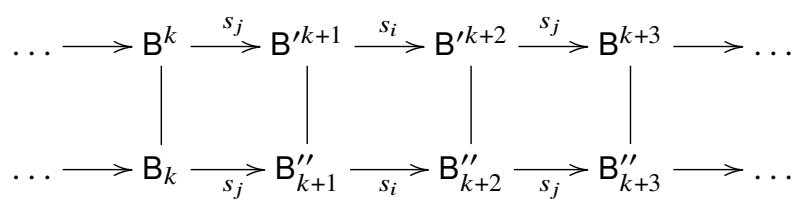

To show that $\mathrm{B}_{k+1}^{\prime}=\mathrm{B}_{k+1}^{\prime \prime}, \mathrm{B}_{k+2}^{\prime}=\mathrm{B}_{k+2}^{\prime \prime}$ and $\mathrm{B}_{k+3}=\mathrm{B}_{k+3}^{\prime \prime}$, it suffices to show $\mathrm{B}_{k+3}=\mathrm{B}_{k+3}^{\prime \prime}$, because the other two follow from the uniqueness part of Lemma 2.5. Note that $w:=s_{i} s_{j} s_{i}=s_{j} s_{i} s_{j}$ is a Weyl group element and during the opposite chain construction, $\mathrm{B}_{k+3}$ and $\mathrm{B}_{k+3}^{\prime \prime}$ are both determined by the relative position relations
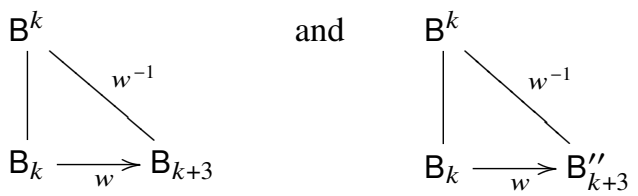

Therefore, we can conclude $\mathrm{B}_{k+3}=\mathrm{B}_{k+3}^{\prime \prime}$ from the uniqueness part of Lemma 2.5. This concludes the proof of the claim that the opposite chain construction is braid move equivariant.

Now after a sequence of braid moves from $\mathbf{i}$ to $\mathbf{j}$, we reach a $\mathbf{j}$-chain $\mathrm{B}(\mathbf{j})=\left(\mathrm{B}^{\prime 0}, \ldots, \mathrm{B}^{\prime n}\right)$ and its opposite chain $\mathrm{B}(\mathbf{j})^{o p}=\left(\mathrm{B}_{0}^{\prime}, \ldots, \mathrm{B}_{n}^{\prime}\right)$. If $i_{k}=j_{k}$ for $m<k \leq n$, we prove $\mathrm{B}^{m}=\mathrm{B}^{\prime m}$ by induction.

For $m=n$, it is clear because braid moves do not change the flags at both ends of the chains. Suppose that inductively we know that $\mathrm{B}^{m+1}=\mathrm{B}^{\prime m+1}$. Applying the inductive hypothesis to the opposite chain under the same sequence of braid moves we have $\mathrm{B}_{m+1}=\mathrm{B}_{m+1}^{\prime}$. Because the opposite sequence construction is braid move equivariant, we know that the bottom primed sequence can be constructed from the top primed chain as an opposite sequence. By construction, $\mathrm{B}^{\prime m}$ is the unique flag such 
that and $\mathrm{B}_{m+1}^{\prime}=\mathrm{B}_{m+1}$. If $i_{m+1}=j_{m+1}$, then $\mathrm{B}^{\prime m}=\mathrm{B}^{m}$ by Lemma 2.5 .

Theorem 2.18 implies that $\tau_{\mathbf{i}}^{\mathbf{j}}$ is canonical and does not depend on the choice of $\tau$. This allows us to replace the word $\mathbf{i}$ by the positive braid $b$.

Definition 2.19. Given a positive braid $b$, the set of $b$-chains of flags is defined as

$$
\mathcal{C}(b)=\bigsqcup_{\mathbf{i} \in \mathbf{H}(b)} \mathcal{C}(\mathbf{i}) /\left(\text { bijections } \tau_{\mathbf{i}}^{\mathbf{j}}\right)
$$

where $\mathbf{i}$ runs through all the words of $b$. We denote a $b$-chain as $\mathrm{B}(b)$. Similarly, let $\mathcal{C}_{ \pm}(b) \subset \mathcal{C}(b)$ be the set of $b$-chains of flags in $\mathcal{B}_{ \pm}$.

Following the proof of Theorem 2.18, braid moves keep the initial and terminal flags of every chain intact. For $\mathrm{B}(b) \in \mathcal{C}(b)$, we denote its initial flag by $\mathrm{B}(b)_{i}$ and its terminal flag by $\mathrm{B}(b)_{t}$. Because braid moves are equivarient under the $\mathrm{G}$-actions on $\mathbf{i}$-chains, one can lift the $\mathrm{G}$-action to $\mathcal{C}(b)$.

Definition 2.20. Let $b$ and $d$ be positive braids. The double Bott-Samelson cell $\operatorname{Conf}_{d}^{b}(\mathcal{B})$ is

$$
\operatorname{Conf}_{d}^{b}(\mathcal{B}):=\mathrm{G} \backslash\left\{(\mathrm{B}(b), \mathrm{B}(d)) \in \mathcal{C}_{+}(b) \times \mathcal{C}_{-}(d) \mid \begin{array}{l}
\mathrm{B}(d)_{i} \text { is opposite to } \mathrm{B}(b)_{i} \\
\mathrm{~B}(d)_{t} \text { is opposite to } \mathrm{B}(b)_{t}
\end{array}\right\} .
$$

After fixing words $\mathbf{i}=\left(i_{1}, i_{2}, \ldots, i_{m}\right)$ and $\mathbf{j}=\left(j_{1}, j_{2}, \ldots, j_{n}\right)$ for $b$ and $d$, one can write a point in $\operatorname{Conf}_{d}^{b}(\mathcal{B})$ as the G-orbit of a collection of flags

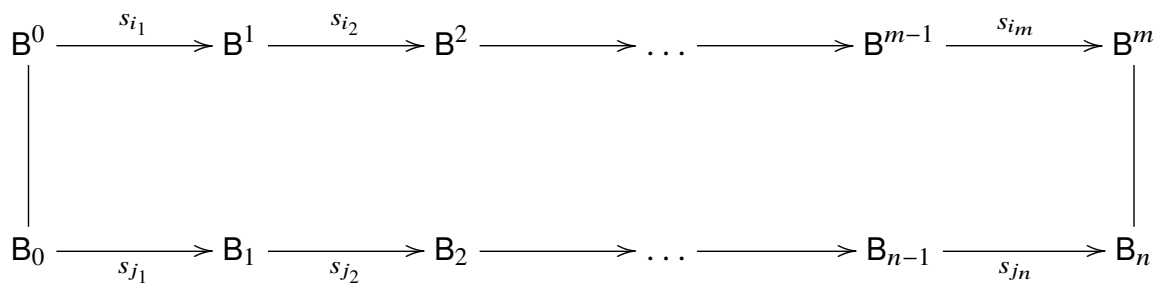

Definition 2.21. The decorated double Bott-Samelson cell $\operatorname{Conf}_{d}^{b}\left(\mathcal{A}_{\mathrm{sc}}\right) \operatorname{or~Conf}_{d}^{b}\left(\mathcal{A}_{\mathrm{ad}}\right)$ is defined to be the moduli space of configurations of flags that are points in $\operatorname{Conf}_{d}^{b}(\mathcal{B})$ together with a decorated flag $\mathrm{A}^{0}$ over $\mathrm{B}^{0}:=\mathrm{B}(b)_{i}$ and a decorated flag $\mathrm{A}_{n}$ over $\mathrm{B}_{n}=\mathrm{B}(d)_{t}$.

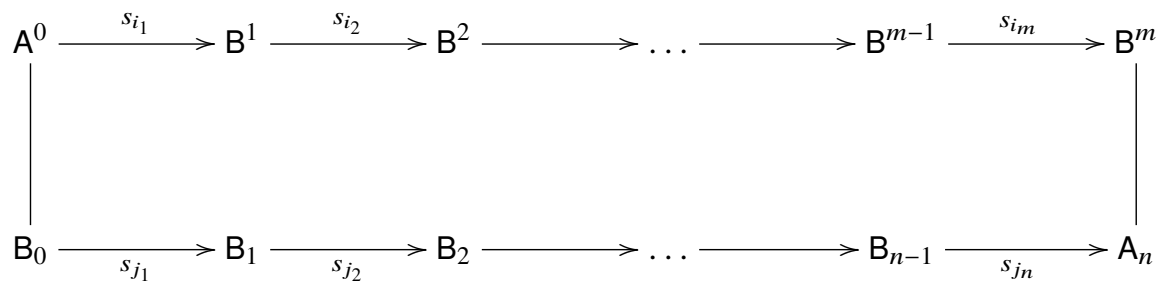

Remark 2.22. Alternatively, one may think of $\operatorname{Conf}_{d}^{b}\left(\mathcal{A}_{\mathrm{sc}}\right)$ as having chains of compatible decorated flags along the horizontal edges, with the top chain determined by $\mathrm{A}^{0}$ and the bottom chain determined by $\mathrm{A}_{n}$, and think of $\operatorname{Conf}_{d}^{b}\left(\mathcal{A}_{\mathrm{ad}}\right)$ as having two pinnings along the vertical edges, with the left pinning 
determined by $\mathrm{A}^{0}$ and the right pinning determined by $\mathrm{A}_{n}$, as shown below. See Subsections 3.2 and 3.3 for more details.
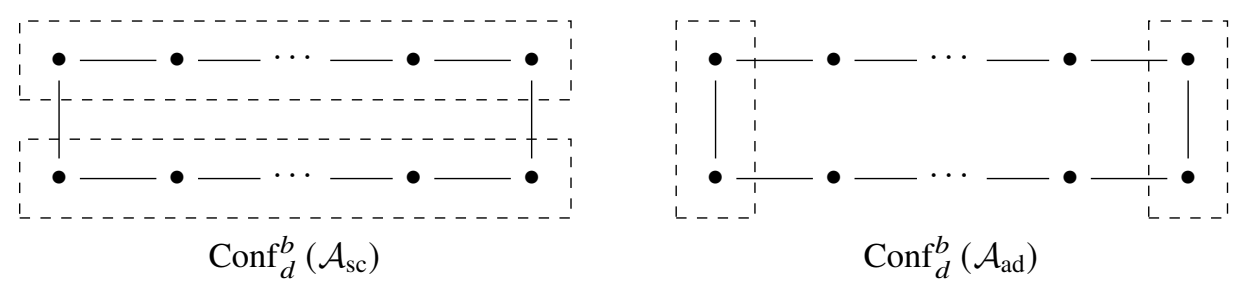

Definition 2.23. The framed double Bott-Samelson cell $\operatorname{Conf}_{d}^{b}\left(\mathcal{A}_{\mathrm{sc}}^{\mathrm{fr}}\right)$ is defined to be the subspace of $\operatorname{Conf}_{d}^{b}\left(\mathcal{A}_{\mathrm{sc}}\right)$ whose decorated flags $\mathrm{A}^{0}$ and $\mathrm{A}_{n}$ induce a decorated flag over each flag via Lemma 2.10 and Corollary 2.14, so that any two consecutive decorated flags on the perimeter are either compatible or form a pinning.

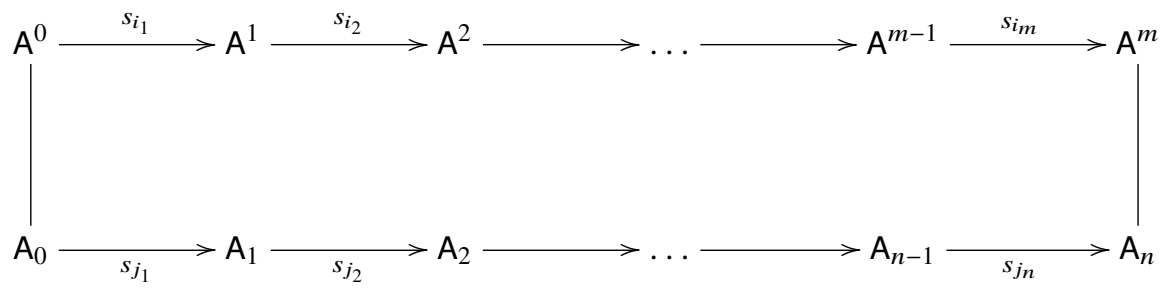

Remark 2.24. Sometimes the indices on the flags in the diagrams of double Bott-Samelson cells may not start from 0 , as we will see in the next subsection.

There are canonical maps

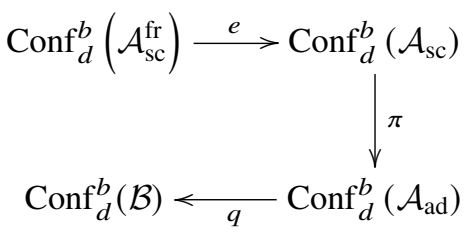

where the maps $e$ and $q$ are forgetful maps and $\pi$ is induced by the projection $\pi: \mathcal{A}_{\mathrm{sc}} \rightarrow \mathcal{A}_{\mathrm{ad}}$.

Proposition 2.26. Let $\mathcal{A}=\mathcal{A}_{\mathrm{sc}}$ or $\mathcal{A}_{\mathrm{ad}}$. The forgetful map $\operatorname{Conf}_{d}^{b}(\mathcal{A}) \rightarrow \operatorname{Conf}_{d}^{b}(\mathcal{B})$ makes $\operatorname{Conf}_{d}^{b}(\mathcal{A}) a$ $\mathrm{T} \times \mathrm{T}$-principal bundle over $\operatorname{Conf}_{d}^{b}(\mathcal{B})$.

Proof. It follows from the fact that T acts freely and transitively on fibres of the projection $\mathcal{A}_{ \pm} \rightarrow \mathcal{B}_{ \pm}$.

\subsection{Reflections and Transposition}

In this section we introduce five natural biregular morphisms between double Bott-Samelson cells: four reflection maps and a transposition map. 
Let us start with the left reflection ${ }_{i} r: \operatorname{Conf}_{s_{i} d}^{b}(\mathcal{B}) \rightarrow \operatorname{Conf}_{d}^{s_{i} b}(\mathcal{B})$. Recall that elements of $\operatorname{Conf}_{s_{i} d}^{b}(\mathcal{B})$ are equivalence classes of collections of flags of the following relative pattern:

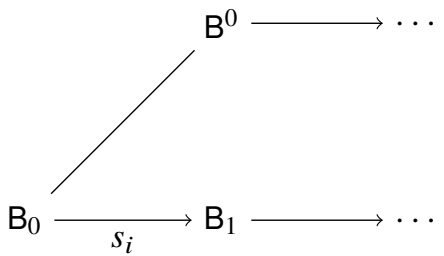

By Lemma 2.5, there is a unique Borel subgroup $B_{-1}$ such that

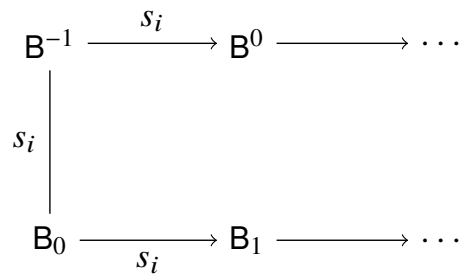

Note that $\left(\mathrm{B}^{-1}, \mathrm{~B}_{1}\right)$ is in general position. Hence, we get a configuration that belongs to $\operatorname{Conf}_{d}^{s_{i} b}(\mathcal{B})$ :

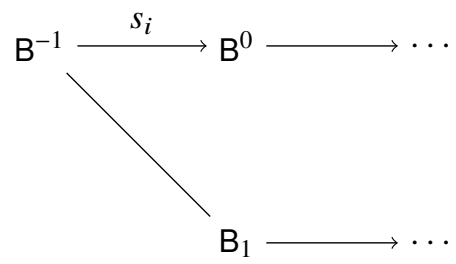

This defines a left reflection

$$
{ }_{i} r: \operatorname{Conf}_{s_{i} d}^{b}(\mathcal{B}) \rightarrow \operatorname{Conf}_{d}^{s_{i} b}(\mathcal{B}) .
$$

One can reverse this construction and get another left reflection that is the inverse of ${ }_{i} r$ :

$$
{ }^{i} r: \operatorname{Conf}_{d}^{s_{i} b}(\mathcal{B}) \rightarrow \operatorname{Conf}_{s_{i} d}^{b}(\mathcal{B}) .
$$

Let us apply the same construction on the right: the flags $\mathrm{B}^{m+1}$ and $\mathrm{B}_{n+1}$ uniquely determine each other and hence we can define two right reflections that are inverse of each other:

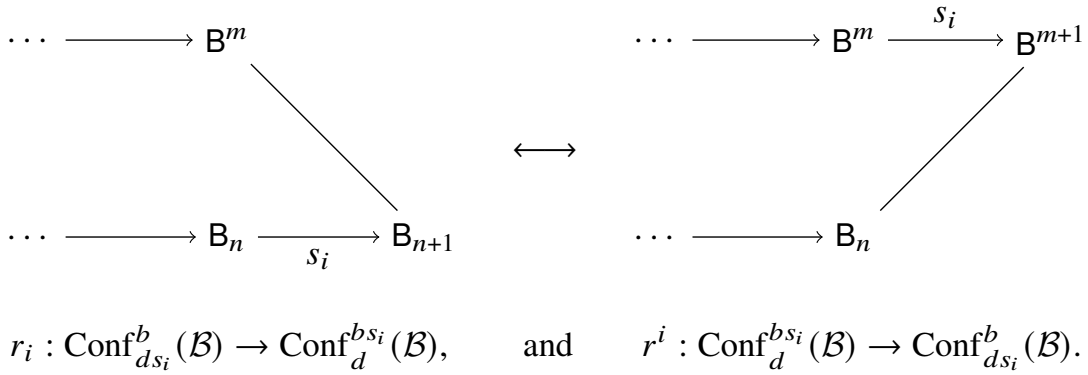

Notation 2.27. We adopt the convention of writing the reflection with the index $i$ at one of the four corners, indicating the double Bott-Samelson cell from which the reflection map originates. 
For $b=s_{i_{1}} s_{i_{2}} \cdots s_{i_{p}} \in \mathrm{Br}_{+}$, we set $b^{\circ}=s_{i_{p}} \ldots s_{i_{2}} s_{i_{1}}$. By composing reflection maps in the four possible directions we get the following four biregular isomorphisms:

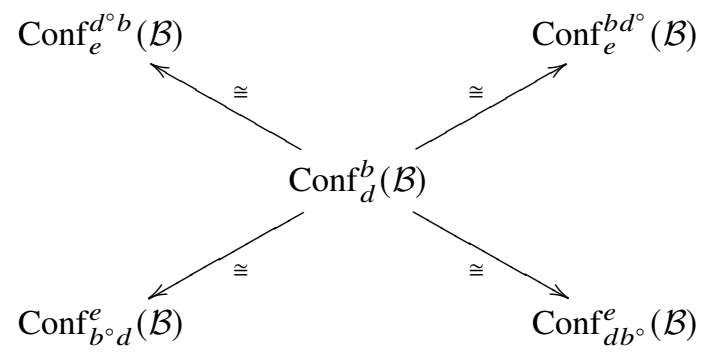

The reflection maps on $\operatorname{Conf}_{d}^{b}(\mathcal{B})$ can be naturally extended to reflection maps on $\operatorname{Conf}_{d}^{b}(\mathcal{A})$. Below we present the construction by using the left reflection ${ }_{i} r$ as an example. Let us start with the configuration on the left. First we reflect $B_{0}$ from the lower left-hand corner to become $B^{-1}$ at the upper left-hand corner; then we find the unique decorated flag $A^{-1}$ over $B^{-1}$ such that $\left(A^{-1}, A^{0}\right)$ is compatible (Lemma 2.10); finally, we forget the decoration of $A^{0}$ and 'downgrade' it to $B^{0}$.
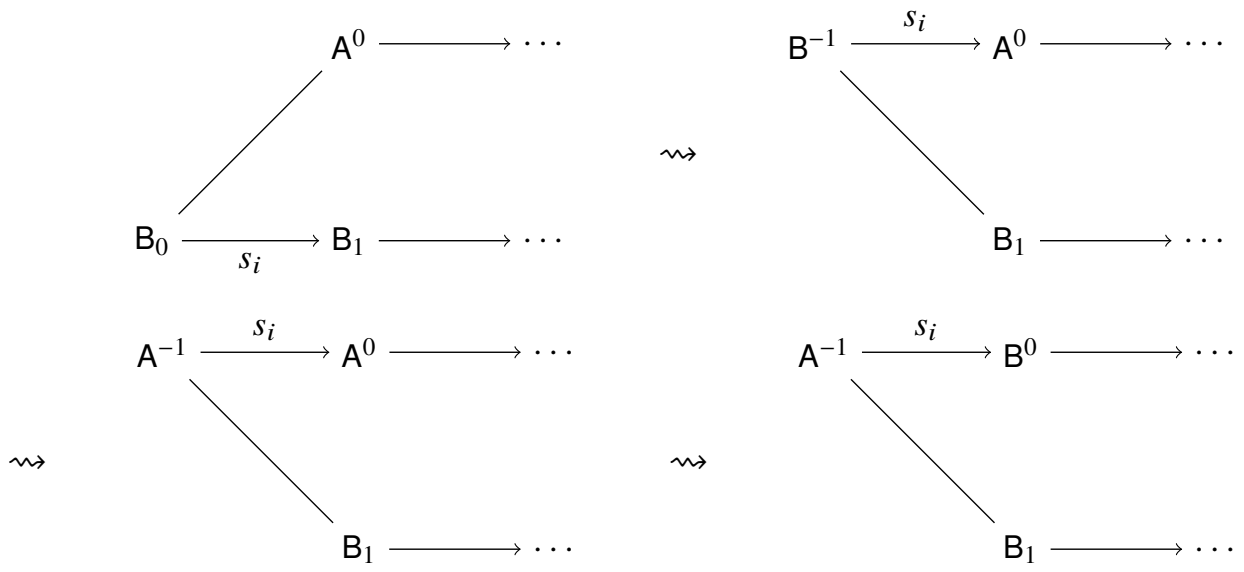

Below is an illustration of the left reflection ${ }^{i} r$. It moves the decoration before reflection.
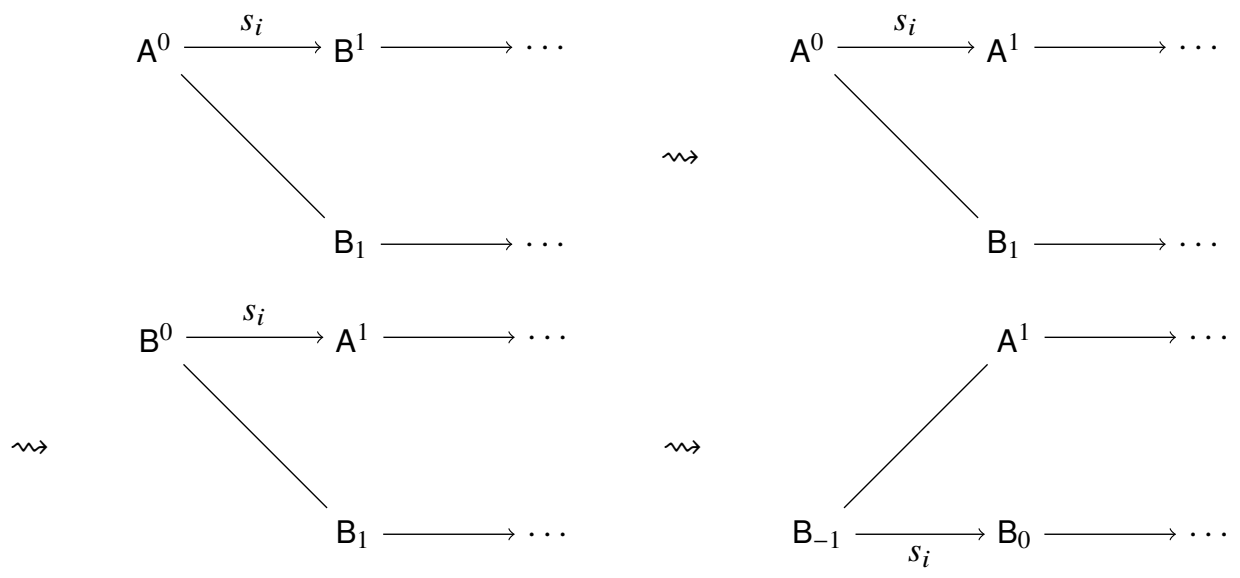

The following lemma implies that the reflection maps can be restricted to $\operatorname{Conf}_{d}^{b}\left(\mathcal{A}_{\mathrm{sc}}^{\mathrm{fr}}\right)$. 
Lemma 2.28. Let $\mathrm{A}_{0} \stackrel{s_{i}}{\longrightarrow} \mathrm{A}_{1}$ and $\mathrm{A}^{0} \stackrel{s_{i}}{\longrightarrow} \mathrm{A}^{1}$ be compatible pairs. If either of

(1) $\mathrm{A}_{0} \stackrel{s_{i}}{\mathrm{~A}^{0}}$

(2) $\mathrm{A}_{1} \stackrel{s_{i}}{-\mathrm{A}^{1}}$

is true, then $\left(\mathrm{A}_{1}, \mathrm{~A}^{0}\right)$ is a pinning if and only if $\left(\mathrm{A}_{0}, \mathrm{~A}^{1}\right)$ is a pinning.

Proof. Due to the symmetry of the cases, it suffices to prove that under (1), $\left(A_{0}, A^{1}\right)$ is a pinning if $\left(A_{1}, A^{0}\right)$ is. Note that by Lemma 2.5, we know that $A_{0}-A^{1}$ and $A_{1}-A^{0}$. By Lemma 2.13 we may assume without loss of generality that $A_{1}=U_{-}$and $A^{0}=U_{+}$. Then Lemma 2.5 together with the compatibility condition on $\mathrm{A}_{0} \stackrel{s_{i}}{\longrightarrow} \mathrm{A}_{1}$ implies that $\mathrm{A}_{0}=\mathrm{U}_{-} \overline{\bar{s}}_{i}$. On the other hand, by Lemma A.6 we know that $\mathrm{B}^{1}=e_{i}(q) \bar{s}_{i} \mathrm{~B}_{+}$and hence $\mathrm{A}^{1}=e_{i}(q) \bar{s}_{i} \mathrm{U}_{+}$. Now to show that $\left(\mathrm{A}_{0}, \mathrm{~A}^{1}\right)$ is a pinning, we just need to notice that

$$
\mathrm{U}_{-} \overline{\bar{s}}_{i} e_{i}(q) \bar{s}_{i} \mathrm{U}_{+}=\mathrm{U}_{-} e_{-i}(-q) \mathrm{U}_{+}=\mathrm{U}_{-} \mathrm{U}_{+} .
$$

By Lemma 2.4 and Lemma 2.15, we obtain biregular isomorphisms $(-)^{t}$, called the transposition maps, from the double Bott-Samelson cells associated to $(b, d)$ to the ones associated to $\left(d^{\circ}, b^{\circ}\right)$, as illustrated below:

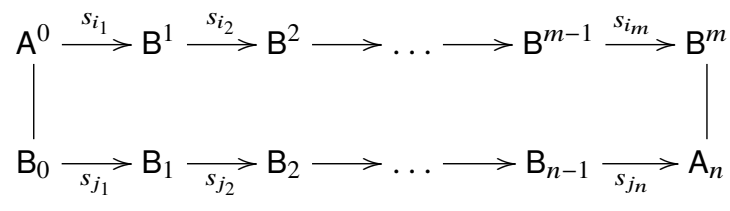

$$
\downarrow(-)^{t}
$$

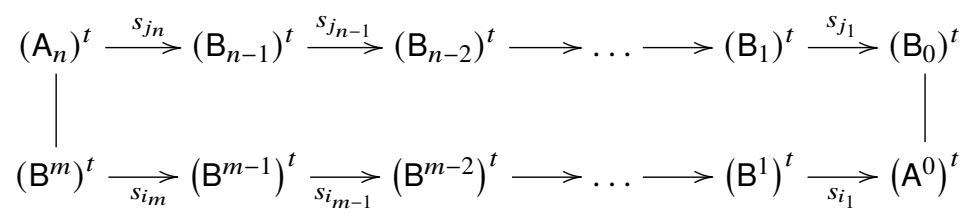

Proposition 2.29. We have the following commutation relations:

(1) $(-)^{t} \circ r_{i}={ }^{i} r \circ(-)^{t}$;

(2) $(-)^{t} \circ r^{i}={ }_{i} r \circ(-)^{t}$;

(3) $(-)^{t} \circ{ }_{i} r=r^{i} \circ(-)^{t}$;

(4) $(-)^{t} \circ{ }^{i} r=r_{i} \circ(-)^{t}$.

Proof. It follows directly from the definitions of the transposition and the reflection morphisms.

\subsection{Affineness of Decorated Double Bott-Samelson Cells}

Theorem 2.30. Both $\operatorname{Conf}_{d}^{b}(\mathcal{A})$ and $\operatorname{Conf}_{d}^{b}\left(\mathcal{A}_{\mathrm{sc}}^{\mathrm{fr}}\right)$ are affine varieties. In particular, $\operatorname{Conf}_{d}^{b}(\mathcal{A})$ is the nonvanishing locus of a single function; hence, it is smooth. 
Proof. Because reflection maps are biregular, it suffices to prove the theorem for the cases when $b=e$. By Corollary 2.13 every element in $\operatorname{Conf}_{d}^{e}(\mathcal{A})$ admits a unique representative that takes the form

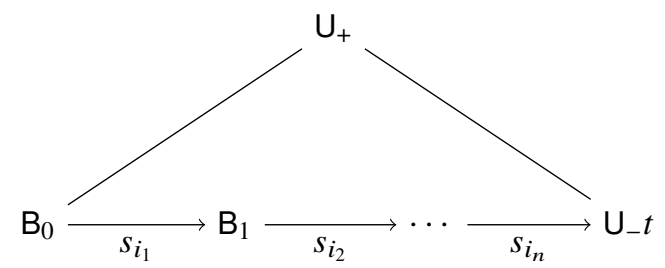

Let us rebuild the above picture by starting with the right edge $U_{+}-U_{-} t$, which is parametrised by the maximal torus $\mathrm{T}$. We find $\mathrm{B}_{n-1}$ that is $s_{i_{n}}$ away from $\mathrm{B}_{-}$. By Corollary A.7, the space of $\mathrm{B}_{n-1}$ is parametrised by $\mathbb{A}^{1}$. Then we proceed to find $\mathrm{B}_{n-2}$ that is $s_{i_{n-1}}$ away from $\mathrm{B}_{n-1}$ and so on until arriving at $\mathrm{B}_{0}$. The parameter space is isomorphic to $T \times \mathbb{A}^{n}$. Let us propagate the decoration from $U_{-} t$ to $B_{k}$ such that every $\left(A_{k}, A_{k-1}\right)$ is compatible:

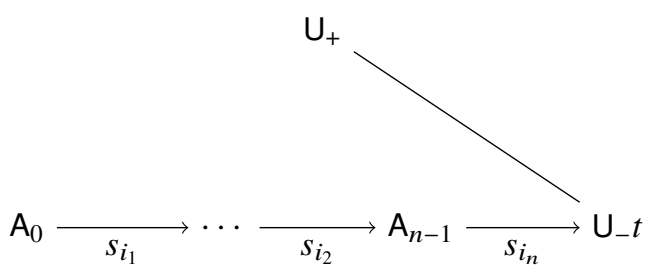

Finally, we require that $\left(A_{0}, U_{+}\right)$is in general position. By Theorem 2.7 and Remark 2.8, it is equivalent to the common nonvanishing locus of a finite collection of regular functions on $T \times \mathbb{A}^{n}$, which is equivalent to a single nonvanishing locus of the product of these regular functions. This concludes the proof of the affineness and the nonvanishing locus nature of $\operatorname{Conf}_{d}^{e}(\mathcal{A})$.

The space $\operatorname{Conf}_{d}^{e}\left(\mathcal{A}_{\mathrm{sc}}^{\mathrm{fr}}\right)$ can be realised as a subvariety of $\operatorname{Conf}_{d}^{e}\left(\mathcal{A}_{\mathrm{sc}}\right)$ by imposing the condition that $\left(U_{+}, U_{-} t\right)$ and $\left(U_{+}, A_{0}\right)$ in the previous picture are pinnings. By Corollary 2.13, it is equivalent to setting certain regular functions to be 1 , which implies the affineness of $\operatorname{Conf}_{d}^{e}\left(\mathcal{A}_{\mathrm{sc}}^{\mathrm{fr}}\right)$.

\section{Cluster Structures on Double Bott-Samelson Cells}

\subsection{Triangulations, String Diagrams and Seeds}

In this section we construct seeds that define the cluster structures on double Bott-Samelson cells. The general definition of a seed can be found in the Appendix. Our main tool is the amalgamation procedure introduced by Fock and Goncharov [FG06].

We start with the definition of a triangulation.

Definition 3.1. Let $(b, d)$ be a pair of positive braids. A triangulation associated to $(b, d)$ is a trapezoid with bases of lengths $m=l(b)$ and $n=l(d)$ together with

(1) a word $\mathbf{i}$ of $b$ whose letters label the unit intervals along the top base of the trapezoid;

(2) a word $\mathbf{j}$ of $d$ whose letters label the unit intervals along the bottom base of the trapezoid;

(3) a collection of line segments called diagonals, each of which connects a marked point on the top to a marked point on the bottom and they divide the trapezoid into triangles. 
Example 3.2. Below is a triangulation associated to the pair of positive braids $\left(s_{1} s_{2} s_{3}, s_{3} s_{1} s_{1} s_{3} s_{2}\right)$.

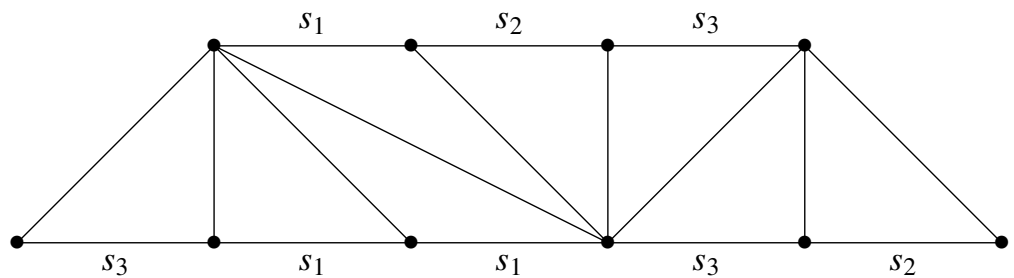

Proposition 3.3. Any two triangulations associated to the positive braids $(b, d)$ can be transformed into one another via a sequence of moves of the following types:

(1) flipping a diagonal within a quadrilateral;

(2) changing the labelling of the unit intervals along the bases locally according to a braid relation.

The construction of seeds involves one more combinatorial gadget called a string diagram, which is obtained from a triangulation as follows:

(1) Lay down $\tilde{r}$ horizontal lines across the trapezoid; call the $i$ th line from the top the ithel.

(2) For each triangle of the form

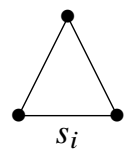
we put a node labeled by $i$ on the $i$ th level within the triangle.

(3) For each triangle of the form

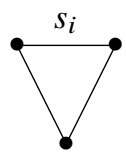

we put a node labeled by $-i$ on the $i$ th level within the triangle.

(4) The nodes cut the horizontal lines into line segments called strings. Strings with nodes at both ends are called closed strings. The remaining strings are called open strings.

Example 3.4. The blue diagram below is the string diagram associated to the triangulation in Example 3.2 with $r=3$ and $l=1$.

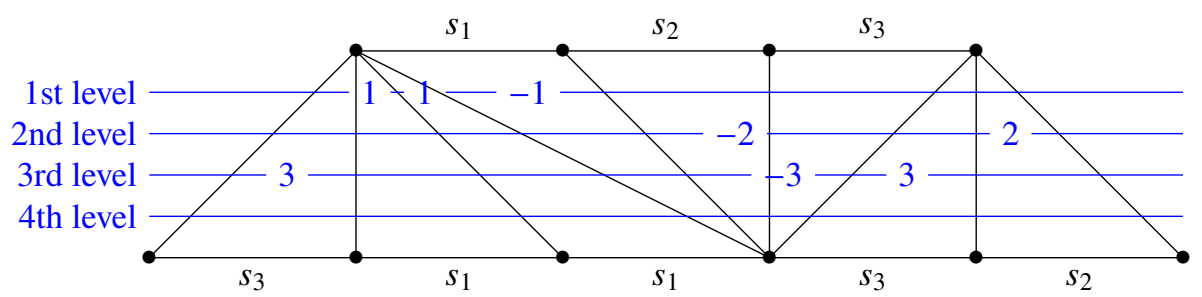

Notation 3.5. We introduce two ways to denote strings in a string diagram: either by a lowercase Latin letter starting from $a, b, c, \ldots$, or by a symbol $\left(\begin{array}{l}i \\ j\end{array}\right)$ with $1 \leq i \leq \tilde{r}$ and $j=0,1,2, \ldots$ The symbol $\left(\begin{array}{l}i \\ j\end{array}\right)$ indicates that it is the $(j+1)$ th string on the $i$ th level counting from the left.

A seed $\mathbf{s}$ consists of a finite set $I$ of vertices, a subset $I^{\mathrm{uf}} \subset I$ of unfrozen vertices, an $I \times I$ matrix $\epsilon$ called the exchange matrix and a collection of positive integers $\left\{d_{a}\right\}_{a \in I}$ called multipliers. The entries of $\epsilon$ are integers unless they are in the submatrix $\left(I \backslash I^{\mathrm{uf}}\right) \times\left(I \backslash I^{\mathrm{uf}}\right)$. We further require that $\operatorname{gcd}\left(d_{a}\right)=1$ and $\hat{\epsilon}_{a b}:=\epsilon_{a b} d_{b}^{-1}$ are skew-symmetric. This requirement resembles the data of the extended generalised symmetrisable Cartan matrix: the $\tilde{r} \times \tilde{r}$ matrix $C$ is equipped with an integral diagonal matrix $\mathrm{D}=\operatorname{diag}\left(\mathrm{D}_{1}, \ldots, \mathrm{D}_{r}, 1, \ldots, 1\right)$ such that $\operatorname{gcd}\left(\mathrm{D}_{i}\right)=1$ and $\mathrm{D}^{-1} \mathrm{C}$ is symmetric.

Now we associate a seed to every string diagram (and hence to every triangulation). Let $I$ be the set of strings and let $I^{\mathrm{uf}}$ be the subset of closed strings. For a string $a$ on the $i$ th level we set $d_{a}:=\mathrm{D}_{i}$ for 
$1 \leq i \leq r$ and $d_{a}:=1$ if $i>r$. The exchange matrix $\epsilon$ is

$$
\epsilon=\sum_{\operatorname{nodes} n} \epsilon^{(n)} .
$$

The matrix $\epsilon^{(n)}$ is defined below for $n$ labelled by $1 \leq i \leq r$. If $n$ is labelled by $-i$, then $\epsilon^{(n)}$ is defined in the same way except that its entries are given by the opposite numbers.

- Let $a$ and $b$ be the two strings on the $i$ th level with the node $n$ as an endpoint as below:

$$
\underline{a} i \stackrel{b}{-}
$$

Then we define

$$
\epsilon_{a b}^{(n)}=-\epsilon_{b a}^{(n)}=-1
$$

- Let $c$ be a string on the $j$ th level that intersects with the triangle that contains $n$. We set

$$
\epsilon_{a c}^{(n)}=-\epsilon_{b c}^{(n)}=-\frac{\mathrm{C}_{j i}}{2}, \quad \epsilon_{c a}^{(n)}=-\epsilon_{c b}^{(n)}=\frac{\mathrm{C}_{i j}}{2} .
$$

$\circ$ The remaining entries are 0 .

Remark 3.6. Although $\epsilon^{(n)}$ have entries with denominator 2, when we sum up all nodes $n$, the entries of the resulting matrix $\epsilon$ are all integers except for those between two open strings.

When we perform the moves in Proposition 3.3, the corresponding mutations of string diagrams and seeds are described in the following proposition. Its proof is a direct combinatorial check and will be skipped. See [FG06, Theorem 3.15].

Proposition 3.7. (1a) If we flip a diagonal inside a quadrilateral $\overbrace{s_{i}}^{s_{s_{j}}}$ with $i \neq j$, the corresponding nodes in the string diagram on different levels will slide across each other and the seed remains the same.

(1b) If we flip a diagonal inside a quadrilateral
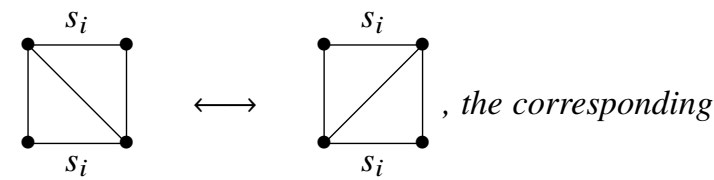

two adjacent nodes in the string diagram are switched and the seed is mutated at the vertex corresponding to the closed string between these two nodes.

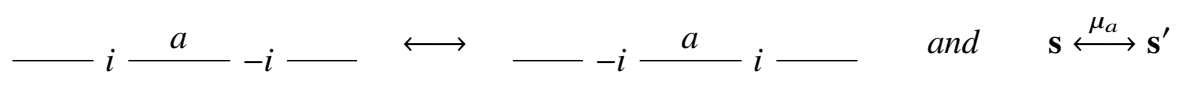

(2) If we perform a braid move to the labelling of the intervals along one of the bases of the trapezoid, depending on whether it is of Dynkin type $\mathrm{A}_{1} \times \mathrm{A}_{1}, \mathrm{~A}_{2}, \mathrm{~B}_{2}$ or $\mathrm{G}_{2}$, the corresponding string diagram undergoes changes as described case by case below and the corresponding seed undergoes a sequence of mutations. We will only depict the cases where a braid move takes place along the bottom base of the trapezoid; the top base cases are completely analogous. 
$\circ \mathrm{C}_{i j}=\mathrm{C}_{j i}=0$ : corresponding nodes slide across each other and the seed remains the same

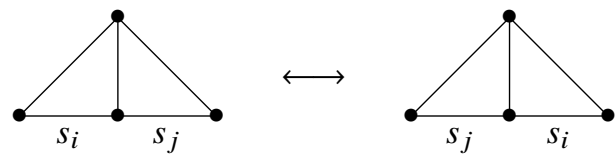

$\circ \mathrm{C}_{i j}=\mathrm{C}_{j i}=-1$ :
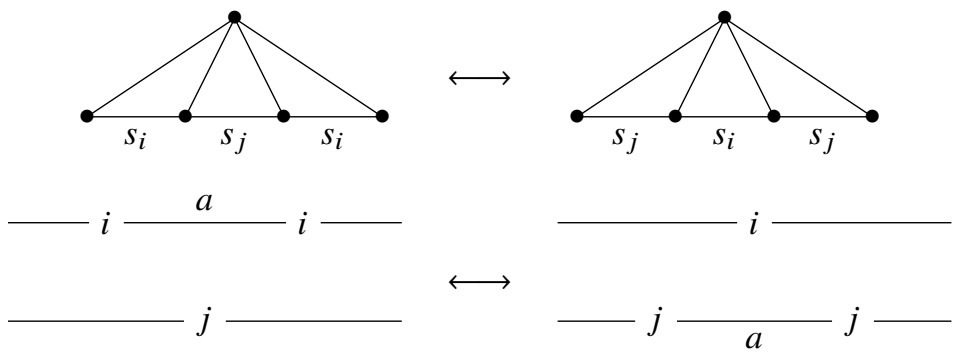

$$
\mathbf{S} \stackrel{\mu_{a}}{\longleftrightarrow} \mathbf{s}^{\prime}
$$

$\circ \mathrm{C}_{i j}=-2$ and $\mathrm{C}_{j i}=-1$ :

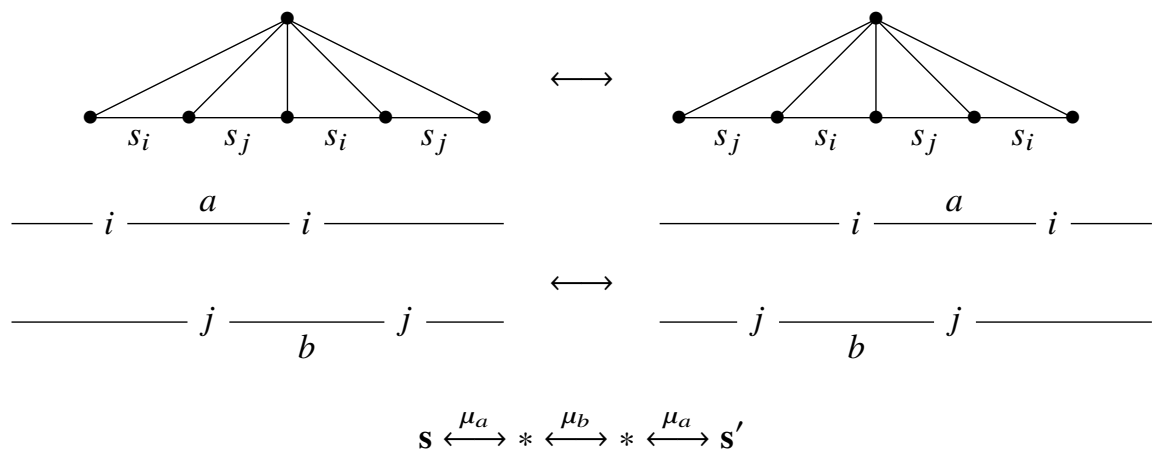

○ $\mathrm{C}_{i j}=-3$ and $\mathrm{C}_{j i}=-1$ :

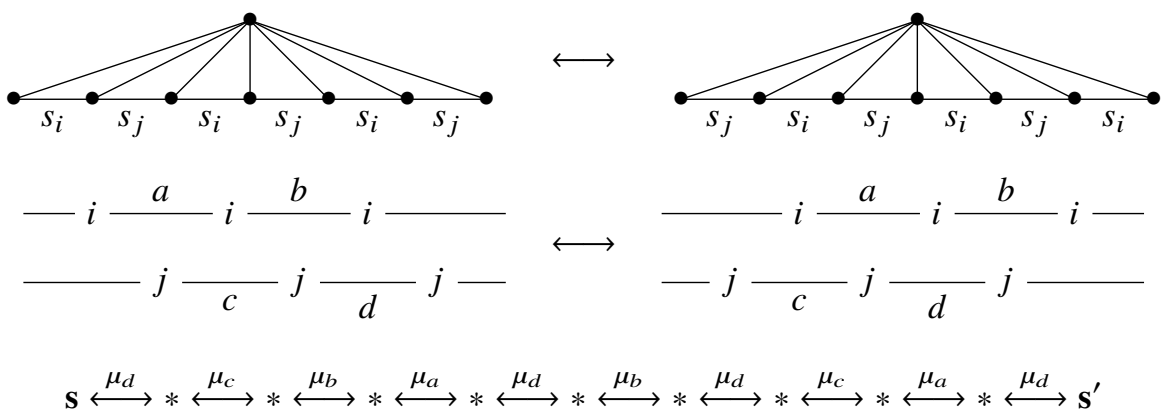

The picture at the end of Section 2 shows that on the combinatorial level the transposition map rotates the trapezoid by 180 degrees. The rotation gives rise to a bijection between the triangulations associated to (b,d) and to (d,b). Correspondingly, there is a bijection between the string diagrams, given by a horizontal flip plus a change of sign for every node. 
Example 3.8. Below are the bijections between triangulations and between string diagrams. Within the two triangulations we point out a pair of corresponding triangles and within the two string diagrams we point out a pair of corresponding strings.

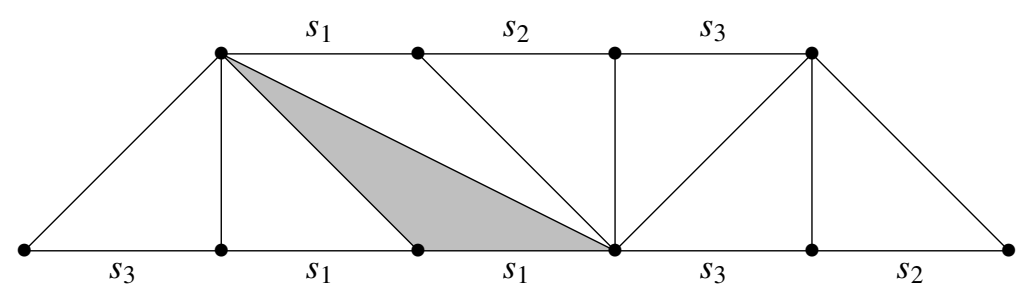

$\uparrow$

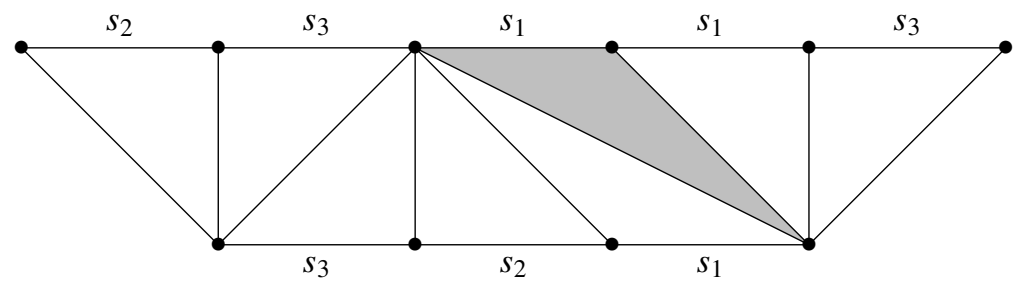

1st level $-1-1-1$

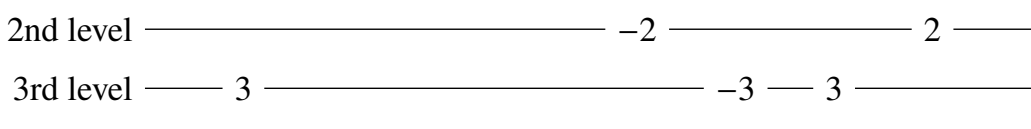

4th level

1st level $-1--1--1$

2nd level $--2-2$

3rd level $--3-3 \longrightarrow-3-$

4th level

Proposition 3.9. Transposition induces a seed isomorphism.

Proof. Note that the local exchange matrix $\epsilon^{(n)}$ is invariant under a simultaneous flip of the strings and changing sign of the nodes. The proposition follows.

It will be proved in later sections that the transposition also induces cluster isomorphisms between the associated cluster ensembles.

\subsection{Cluster Poisson Structure on $\operatorname{Conf}_{d}^{b}\left(\mathcal{A}_{\mathrm{ad}}\right)$ and $\operatorname{Conf}_{d}^{b}(\mathcal{B})$}

In this section we associate a coordinate chart of $\operatorname{Conf}_{d}^{b}\left(\mathcal{A}_{\text {ad }}\right)$ to every seed $\mathbf{s}$ obtained from a triangulation. We show that these coordinate charts are related by the cluster Poisson mutations corresponding to the seed mutations in Proposition 3.7, equipping $\operatorname{Conf}_{d}^{b}\left(\mathcal{A}_{\mathrm{ad}}\right)$ with a natural cluster Poisson structure. The space $\operatorname{Conf}_{d}^{b}(\mathcal{B})$ inherits a cluster Poisson structure from that of $\operatorname{Conf}_{d}^{b}\left(\mathcal{A}_{\mathrm{ad}}\right)$ via the projection $\operatorname{Conf}_{d}^{b}\left(\mathcal{A}_{\mathrm{ad}}\right) \rightarrow \operatorname{Conf}_{d}^{b}(\mathcal{B})$. 
Recall that points in $\operatorname{Conf}_{d}^{b}\left(\mathcal{A}_{\mathrm{ad}}\right)$ are configurations of flags of the following form:

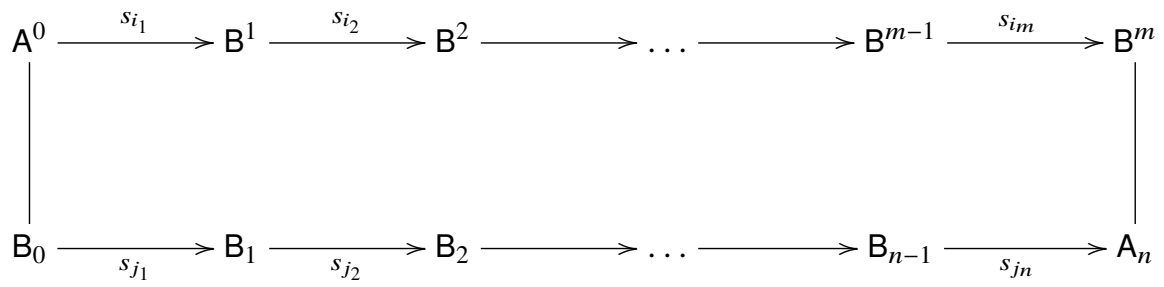

When we draw certain diagonals on the trapezoid to make a triangulation, we can view each of the extra diagonals as imposing a general position condition on the underlying undecorated flags it connects. The coordinate system we construct will depend on a choice of such triangulation.

By Corollary 2.14 we know that the left edge $B_{0} \longrightarrow A^{0}$ is equivalent to a pinning and by Lemma 2.13 we may use the $\mathrm{G}$-action to move the whole configuration to a unique representative with $\mathrm{B}_{0}=\mathrm{B}_{-}$ and $A^{0}=U_{+}$. We call such a unique representative the special representative and we will use it heavily for the discussion below. Note that in this particular representative, the underlying undecorated flag of $A^{0}$ is $B_{+}$. The key lemma to define the cluster Poisson coordinates is the following.

Lemma 3.10. Fix the special representative with $\mathrm{A}^{0}=\mathrm{U}_{+}$and $\mathrm{B}_{0}=\mathrm{B}_{-}$. One can associate a unique unipotent element $e_{-i}(p)$ to each triangle of the form

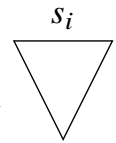
and a unique unipotent element $e_{i}(q)$ to each triangle of the form $\bigwedge_{s_{i}}$, with $p, q \neq 0$, such that for any diagonal $\mathrm{B}_{k}-\mathrm{B}^{l}$ in the triangulation (including the rightmost edge $\mathrm{B}_{n} \longrightarrow \mathrm{B}^{m}$ ),

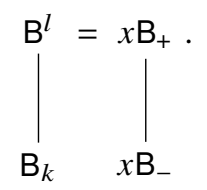

where $x$ is the product of unipotent elements $e_{-i}(p)$ and $e_{i}(q)$ are associated to triangles to the left of the diagonal according to their order from left to right on the triangulation.

Example 3.11. Let us convey the meaning of the above lemma in a concrete example. Consider the following triangulation. Suppose we have fixed the special representative with $A^{0}=U_{+}$and $B_{0}=B_{-}$. Then the lemma claims that there exist unique unipotent elements $e_{-i}(p)$ and $e_{i}(q)$ associated to the triangles (with $p, q \neq 0$ ), such that any pair of undecorated flags connected by a diagonal can be obtained by moving the pair $\left(B_{-}, B_{+}\right)$by a group element that is the product of those unipotent elements to the left of that diagonal.

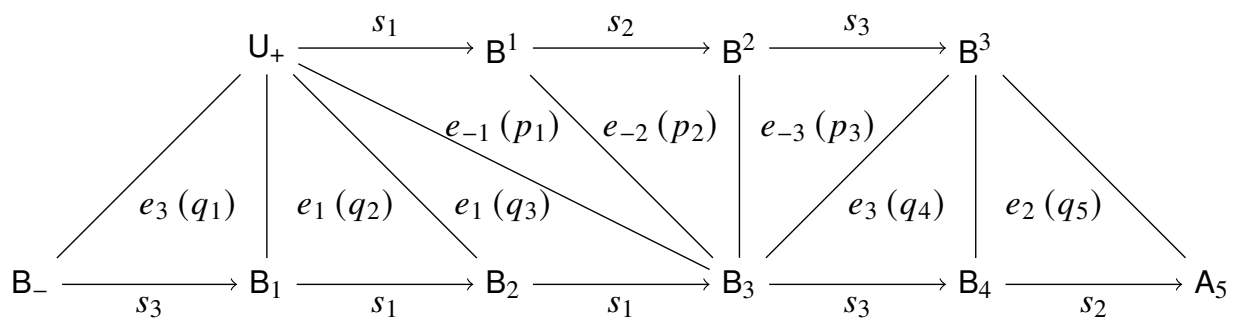


For example, $\left.\right|_{\mathrm{B}_{3}} ^{\mathrm{B}^{1}}=\left.\right|_{x \mathrm{~B}_{-}} ^{x \mathrm{~B}_{+}}$with $x=e_{3}\left(q_{1}\right) e_{1}\left(q_{2}\right) e_{1}\left(q_{3}\right) e_{-1}\left(p_{1}\right)$, and $\left.\right|_{\mathrm{B}_{4}} ^{\mathrm{B}^{3}}=\left.\right|_{y \mathrm{~B}_{-}} ^{y \mathrm{~B}_{+}}$with $y=$ $e_{3}\left(q_{1}\right) e_{1}\left(q_{2}\right) e_{1}\left(q_{3}\right) e_{-1}\left(p_{1}\right) e_{-2}\left(p_{2}\right) e_{-3}\left(p_{3}\right) e_{3}\left(q_{4}\right)$.

Proof of Lemma 3.10. Let us first look at the leftmost triangle. Without loss of generality, we may assume that it is of the following shape (the upside-down case is similar):

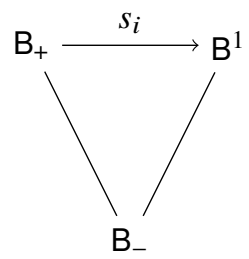

We would like to show that $\mathrm{B}^{1}=e_{-i}(p) \mathrm{B}_{+}$for some unique $p \neq 0$. Suppose that $\mathrm{B}_{1}=x \mathrm{~B}_{+}$. Then from $\mathrm{B}_{-}-x \mathrm{~B}_{+}$we know that $x$ is Gaussian decomposable; that is, $x=[x]_{-}[x]_{0}[x]_{+}$. In particular, $x \mathrm{~B}_{+}=[x]_{-} \mathrm{B}_{+}$, so without loss of generality we may replace $x$ by $[x]_{-}$and assume that $x \in \mathrm{U}_{-}$. Now the top edge also tells us that $x \in \mathrm{B}_{+} s_{i} \mathrm{~B}_{+}$. But $\mathrm{U}_{-} \cap \mathrm{B}_{+} s_{i} \mathrm{~B}_{+}=\cup_{-i}=\left\{e_{-i}(p)\right\} \cong \mathbb{A}_{p}^{1}$. This shows that $x=e_{-i}(p)$ for some $p$ and we know that $p \neq 0$ because $\mathrm{B}_{1} \neq \mathrm{B}_{+}$. Also note that for different values of $p, e_{-i}(p) \mathrm{B}_{+}$are distinct flags. Therefore, $\mathrm{B}^{1}=e_{-i}(p) \mathrm{B}_{+}$for some unique $p \neq 0$.

Now we move on to the next triangle. But instead of doing a new argument, we can move the whole configuration by $e_{-i}(-p)$ so that $\mathrm{B}^{1}$ becomes $\mathrm{B}_{+}$and then repeat the same argument above. To be more precise, let us suppose that the second triangle looks like the following:

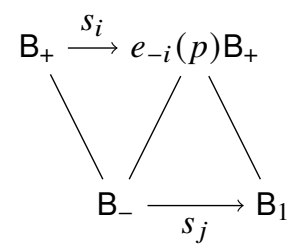

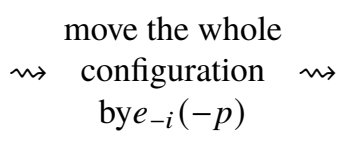

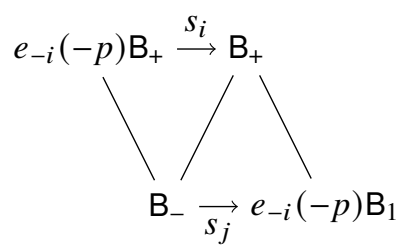

Then by applying the same argument to the second triangle, we get $e_{-i}(-p) \mathrm{B}_{1}=e_{j}(q) \mathrm{B}_{-}$and hence $\mathrm{B}_{1}=e_{-i}(p) e_{j}(q) \mathrm{B}_{-}$.

We can repeat the argument again for the third triangle by first moving the special representative by $\left(e_{-i}(p) e_{j}(q)\right)^{-1}$ and similarly for the fourth triangle and so on. Each step will produce a unique unipotent element as required by the lemma.

Definition 3.12. The nonzero numbers $p_{i}$ and $q_{i}$ are called Lusztig factorisation coordinates.

From the construction of $\mathrm{G}_{\mathrm{ad}}$ (see Appendix A.1 for more details), we have a basis $\left\{\omega_{i}^{\vee}\right\}_{i=1}^{\tilde{r}}$ for the cocharacter lattice of the maximal torus $\mathrm{T}_{\mathrm{ad}}$ and we can factor the unipotent elements $e_{i}(q)$ and $e_{-i}(p)$ as

$$
e_{i}(q)=q^{\omega_{i}^{\vee}} e_{i} q^{-\omega_{i}^{\vee}} \quad \text { and } \quad e_{-i}(p)=p^{-\omega_{i}^{\vee}} e_{-i} p^{\omega_{i}^{\vee}}
$$

Therefore, instead of multiplying the unipotent group elements $e_{-i}(p)$ and $e_{j}(q)$ according to their order in the triangulation, we can split each of these unipotent group elements into three factors and associate them to different parts of the corresponding string diagram. More precisely, we associate the 
maximal torus elements with strings and associate $e_{ \pm i}$ with the nodes $\pm i$.

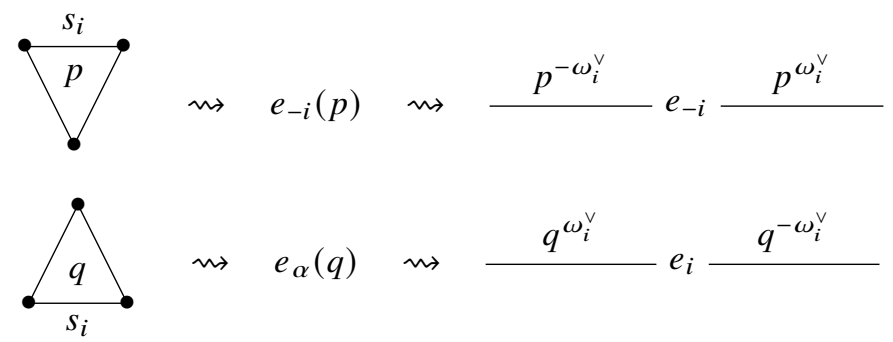

We then make the following observation. To a closed string $a$ on the $i$ th level there are two maximal torus elements attached, one at each end; we can multiply them and get a maximal torus element of the form $X_{a}^{\omega_{i}^{\vee}}$. To an open string $a$ on the $i$ th level on the left side of the string diagram there is one maximal torus element attached and we can put it in the form $X_{a}^{\omega_{i}^{\vee}}$. Note that these numbers $X_{a}$ are ratios of Lusztig factorisation coordinates and hence they are nonzero.

Moreover, any ordered product of unipotent group elements on the triangulation diagram is equal to an ordered product of maximal torus elements of the form $X_{a}^{\omega_{i}^{\vee}}$ associated to the strings and the Chevalley generators $e_{ \pm i}$ associated to the nodes within the corresponding part of the string diagram, according to their order on the string diagram. Note that because $X^{\omega_{i}^{\vee}} e_{ \pm j}=e_{ \pm j} X^{\omega_{i}^{\vee}}$ whenever $i \neq j$, ambiguous ordering between factors on different levels does not affect the outcome of the products.

However, the Lusztig factorisation coordinates are not enough to define the cluster Poisson coordinates. After finding all of the Lusztig factorisation coordinates, we can multiply all of the unipotent elements associated to the triangles together according to the order of the triangulation and get a group element $g$, and by Lemma 3.10, the rightmost edge of the special representative is $\left.\right|_{g \mathrm{~B}_{-}}$. Because $\mathrm{A}_{n}$ is a decoration over $\mathrm{B}_{n}=g \mathrm{~B}_{-}$, there must be some $t \in \mathrm{T}_{\text {ad }}$ such that $\mathrm{A}_{n}=\mathrm{U}_{-} t^{-1} g^{-1}$. Furthermore, because $\left\{\omega_{i}^{\vee}\right\}_{i=1}^{\tilde{r}}$ is a basis for the cocharacter lattice of $\mathrm{T}_{\text {ad }}$, we can write $t=\prod_{i=1}^{\tilde{r}} t_{i}^{\omega_{i}^{\vee}}$ for $t_{i} \in \mathbb{G}_{m}$.

Now for an open string $a$ on the $i$ th level on the right side of the string diagram, there may be one maximal torus element coming from its left endpoint, which we can write as $r_{i}^{\omega_{i}^{\vee}}$. We then define $X_{a}:=r_{i} t_{i} \in \mathbb{G}_{m}$ to be the number we associate to this open string $a$.

Definition 3.13. We call the numbers $X_{a}$ the cluster Poisson coordinates associated to the seed (string diagram/triangulation) on $\operatorname{Conf}_{d}^{b}\left(\mathcal{A}_{\mathrm{ad}}\right)$.

Example 3.14. Let us demonstrate the construction of the cluster Poisson coordinates associated to the triangulation in Example 3.11. Remember that we have $r=3$ and $l=1$.

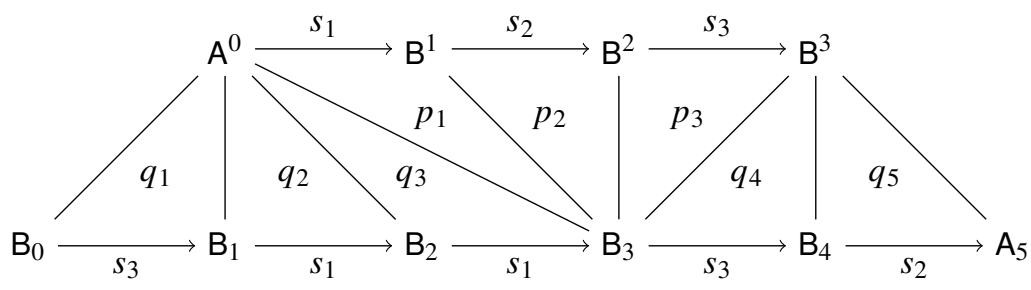

The string diagram of this triangulation is given in Example 3.4. By factoring the unipotent group elements and taking in the extra factor of $t=\prod_{i} t_{i}^{\omega_{i}^{\vee}}$ induced by the decoration $\mathrm{A}_{n}$, we get the following 
group elements associated to strings and nodes of the string diagram:

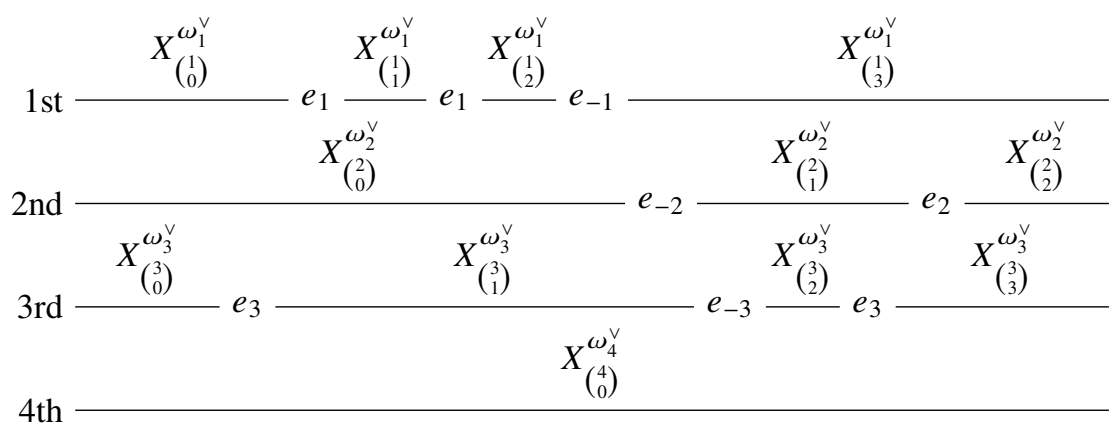

where

$$
\begin{array}{llll}
X_{\left(\begin{array}{l}
1 \\
0
\end{array}\right)}=q_{2} & X_{\left(\begin{array}{l}
1 \\
1
\end{array}\right)}=\frac{q_{3}}{q_{2}} & X_{\left(\begin{array}{c}
1 \\
2
\end{array}\right)}=\frac{1}{q_{3} p_{1}} & X_{\left(\begin{array}{l}
1 \\
3
\end{array}\right)}=p_{1} t_{1} \\
X_{\left(\begin{array}{c}
2 \\
0
\end{array}\right)}=\frac{1}{p_{2}} & X_{\left(\begin{array}{l}
2 \\
1
\end{array}\right)}=p_{2} q_{5} & X_{\left(\begin{array}{l}
2 \\
2
\end{array}\right)}=\frac{t_{2}}{q_{5}} & X_{\left(\begin{array}{c}
3 \\
3
\end{array}\right)}=\frac{t_{3}}{q_{4}} \\
X_{\left(\begin{array}{c}
3 \\
0
\end{array}\right)}=q_{1} & X_{\left(\begin{array}{l}
3 \\
1
\end{array}\right)}=\frac{1}{q_{1} p_{3}} & X_{\left(\begin{array}{c}
3 \\
2
\end{array}\right)}=p_{3} q_{4} & \\
X_{\left(\begin{array}{c}
4 \\
0
\end{array}\right)}=t_{4} . & & &
\end{array}
$$

Note that we can recover all of the Lusztig factorisation coordinates from the cluster Poisson coordinates and hence we can determine all of the underlying undecorated flags in the special representative with $\left.\right|_{\mathrm{B}_{0}} ^{\mathrm{A}^{0}}=\left.\right|_{\mathrm{B}_{-}} ^{\mathrm{A}_{+}}$using the cluster Poisson coordinates.

Recall that the transposition map induces a natural bijection between string diagrams of $(b, d)$ and string diagrams of $\left(d^{\circ}, b^{\circ}\right)$ and a natural bijection between strings inside the two corresponding string diagrams, as well as a seed isomorphism between the associated seeds. Now we would like to lift such correspondence to the level of cluster Poisson coordinate charts.

Proposition 3.15. Let $\left(X_{a}\right)$ and $\left(X_{a}^{\prime}\right)$ be the cluster Poisson coordinate charts associated to two corresponding string diagrams under transposition. Then under the transposition morphism $t$ : $\operatorname{Conf}_{d}^{b}\left(\mathcal{A}_{\mathrm{ad}}\right) \rightarrow \operatorname{Conf}_{b^{\circ}}^{d^{\circ}}\left(\mathcal{A}_{\mathrm{ad}}\right), t^{*} X_{a}^{\prime}=X_{a}$.

Proof. Because cluster Poisson coordinates are computed using Lusztig factorisation coordinates, let us first take a look at how transposition changes Lusztig factorisation coordinates. Let $g$ be the group element that is the ordered product of the unipotent elements associated to the triangles and let $t=\prod_{i=1}^{\tilde{r}} t_{i}^{\omega_{i}^{\vee}}$ be the maximal torus element used to make $A_{n}=U_{-} t^{-1} g^{-1}$. Without loss of generality, we may assume that the last triangle in the special representative is of the following form:

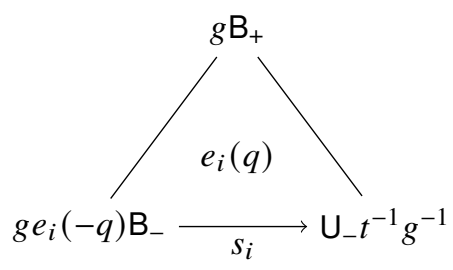


Then under transposition, this triangle is mapped to the following triangle (here $\stackrel{t g^{t}}{m}$ means move the whole configuration by $\operatorname{tg}^{t}$ ):

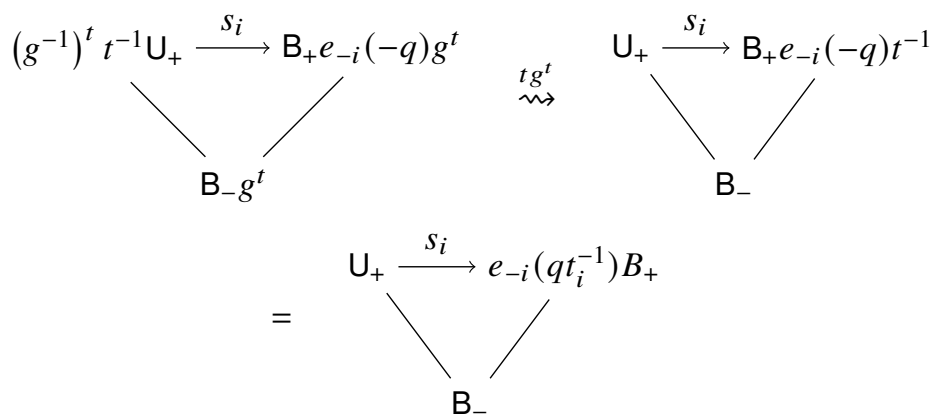

Therefore, the change of Lusztig factorisation coordinate for such configuration is $q \mapsto q t_{i}^{-1}$. By similar computations, it is not hard to find that under transposition, the Lusztig factorisation coordinates for corresponding triangles in the two triangulations are related by

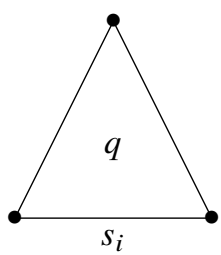

transposition
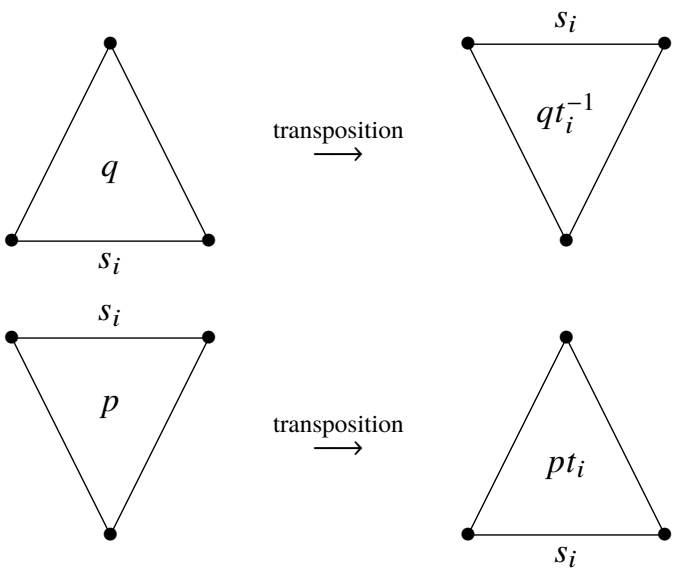

Because the cluster Poisson coordinates associated to the closed strings are of the form $p / p, q / q$, $p q$ or $(p q)^{-1}$, they do not change under transposition as a result of the formulas above.

Let $a^{\prime}$ be an open string on the $i$ th level on the left of the string diagram after transposition. Then $a^{\prime}$ corresponds to an open string $a$ on the $i$ th level on the right of the string diagram before transposition. If the right endpoint of $a^{\prime}$ is a node $i$, then the left endpoint of $a$ is a node $-i$. Let $p$ be the Lusztig factorisation coordinate associated to this node $-i$. From the construction of cluster Poisson coordinates and the coordinate transformation formula for Lusztig factorisation coordinates above, we see that

$$
X_{a}=p t_{i}=X_{a^{\prime}}
$$

On the other hand, if the right endpoint of $a^{\prime}$ is a node $-i$, then the left endpoint of $a$ is a node $i$. Let $q$ be the Lusztig coordinate associated to this node $i$. Then again we have

$$
X_{a}=q^{-1} t_{i}=X_{a^{\prime}} .
$$

Because if we apply transposition twice we get back the identity map, it follows that $t^{*} X_{a^{\prime}}=X_{a}$ also holds for open strings $a^{\prime}$ on the right. This finishes the proof.

Corollary 3.16. The cluster Poisson coordinates associated to closed strings are unchanged under a change of decorations over $\mathrm{B}^{0}$ and $\mathrm{B}_{n}$. 
Proof. From the construction of cluster Poisson coordinates it is obvious that the ones associated to closed strings are unaffected by a change of decoration on $\mathrm{B}_{n}$. But then because the roles of $\mathrm{B}^{0}$ and $\mathrm{B}_{n}$ are interchanged under transposition and the cluster Poisson coordinates remain unchanged under transposition, we can deduce that the cluster Poisson coordinates associated to the closed strings are unaffected by a change of decoration on $\mathrm{B}^{0}$.

This corollary shows that the subset of cluster Poisson coordinates associated to closed strings actually descend to coordinates on $\operatorname{Conf}_{d}^{b}(\mathcal{B})$. We call these coordinates the cluster Poisson coordinates on $\operatorname{Conf}_{d}^{b}(\mathcal{B})$ associated to the seed (string diagram/triangulation).

In order to justify the name 'cluster Poisson coordinates', we need to show that they actually transform as cluster Poisson coordinates, which boils down to showing the cluster Poisson analogue of Proposition 3.7.

\section{Proposition 3.17.}

(1) If $i \neq j$, then

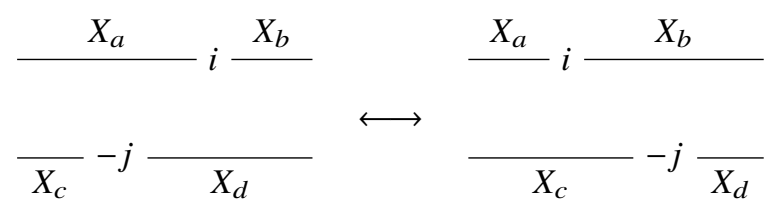

On the other hand, if we interchange two neighbouring nodes of opposite signs on the same level, we get

$$
j \text { ith level level } \frac{X_{a}}{X_{d}}-i \frac{X_{b}}{i \frac{X_{c}}{\longleftrightarrow}} \longleftrightarrow \quad \text { j-level } \frac{X_{a}^{\prime}}{i \frac{X_{b}^{\prime}}{X_{d}^{\prime}}-i \frac{X_{c}^{\prime}}{X_{d}^{\prime}}}
$$

where

$$
\begin{gathered}
X_{a}^{\prime}=X_{a} X_{b}\left(1+X_{b}\right)^{-1}, \quad X_{b}^{\prime}=X_{b}^{-1}, \\
X_{c}^{\prime}=X_{c} X_{b}\left(1+X_{b}\right)^{-1}, \quad X_{d}^{\prime}=X_{d}\left(1+X_{b}\right)^{-C_{i j}} .
\end{gathered}
$$

(2) If we perform a braid move to the string diagram, depending on whether it is of Dynkin type $\mathrm{A}_{1} \times \mathrm{A}_{1}$, $\mathrm{A}_{2}, \mathrm{~B}_{2}$ or $\mathrm{G}_{2}$, the cluster Poisson coordinates transform according to the sequences of mutations described in Proposition 3.7 (we will only depict the case where a braid move takes place among nodes labelled by simple roots; the cases where they are labelled by opposite simple roots are completely analogous).

○ $\mathrm{C}_{i j} \mathrm{C}_{j i}=0$ :

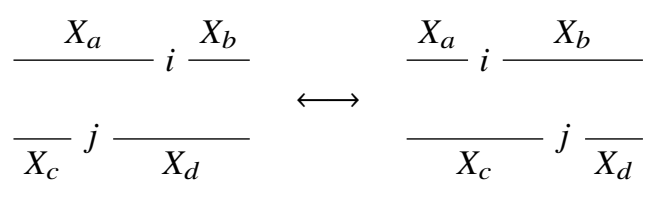

$\circ \mathrm{C}_{i j} \mathrm{C}_{j i}=-1$ :

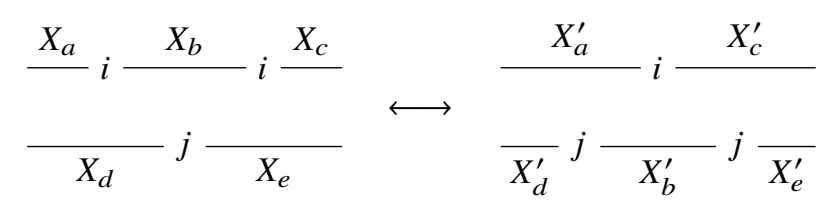


where

$$
\begin{gathered}
X_{a}^{\prime}=X_{a}\left(1+X_{b}\right), \quad X_{b}^{\prime}=X_{b}^{-1}, \quad X_{c}^{\prime}=X_{c} X_{b}\left(1+X_{b}\right)^{-1}, \\
X_{d}^{\prime}=X_{d} X_{b}\left(1+X_{b}\right)^{-1}, \quad X_{e}^{\prime}=X_{e}\left(1+X_{b}\right) .
\end{gathered}
$$

$\circ \mathrm{C}_{i j}=-2$ and $\mathrm{C}_{j i}=-1$ :

$$
\frac{\frac{X_{c}}{X_{e}} j \frac{X_{a}}{X_{b}} j \frac{X_{d}}{X_{f}}}{\longleftrightarrow} \frac{\frac{X_{c}^{\prime}}{X_{e}^{\prime}} j \frac{X_{a}^{\prime}}{X_{b}^{\prime}} j \frac{X_{d}^{\prime}}{X_{f}^{\prime}}}{\longleftrightarrow-\frac{1}{X^{\prime}}}
$$

where

$$
\begin{array}{lll}
X_{a}^{\prime}=X_{a} \frac{1}{F_{b}} & X_{b}^{\prime}=\frac{1}{X_{a}^{2} X_{b}} F_{a}^{2} & X_{c}^{\prime}=X_{c} \frac{F_{b}}{F_{a}} \\
X_{d}^{\prime}=X_{d} F_{a} & X_{e}^{\prime}=X_{e} X_{a}^{2} X_{b} \frac{1}{F_{b}} & X_{f}^{\prime}=X_{f} X_{b} \frac{F_{b}}{F_{a}^{2}}
\end{array}
$$

and

$$
F_{a}=1+X_{b}+X_{a} X_{b} \quad F_{b}=1+X_{b}+2 X_{a} X_{b}+X_{a}^{2} X_{b} .
$$

○ $\mathrm{C}_{i j}=-3$ and $\mathrm{C}_{j i}=-1$ :

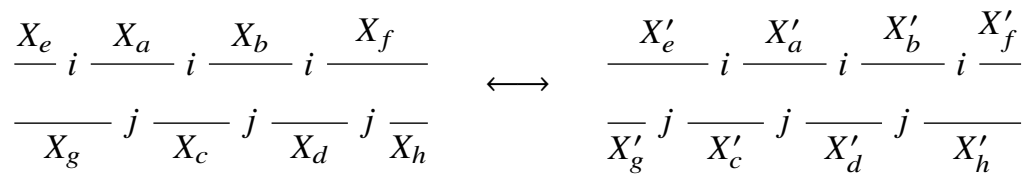

where

$$
\begin{array}{llll}
X_{a}^{\prime}=X_{a} \frac{F_{d}}{F_{b} F_{c}} & X_{b}^{\prime}=X_{b} \frac{F_{a}}{F_{d}} & X_{c}^{\prime}=\frac{1}{X_{a}^{3} X_{b}^{3} X_{c}^{2} X_{d}} \frac{F_{a}^{3}}{F_{d}} & X_{d}^{\prime}=X_{c} \frac{F_{b}^{3} F_{c}}{F_{a}^{3}} \\
X_{e}^{\prime}=X_{e} \frac{F_{c}}{F_{a}} & X_{f}^{\prime}=X_{f} F_{b} & X_{g}^{\prime}=X_{a}^{3} X_{b}^{3} X_{c}^{2} X_{d} X_{g} \frac{1}{F_{c}} & X_{h}^{\prime}=X_{d} X_{h} \frac{F_{d}}{F_{b}^{3}}
\end{array}
$$

and

$$
\begin{aligned}
F_{a}= & +X_{d}+3 X_{b} X_{d}+3 X_{b}^{2} X_{d}+3 X_{b}^{2} X_{c} X_{d}+X_{b}^{3} X_{d}+2 X_{b}^{3} X_{c} X_{d}+X_{b}^{3} X_{b}^{2} X_{d} \\
& +2 X_{a} X_{b}^{2} X_{c} X_{d}+2 X_{a} X_{b}^{3} X_{c} X_{d}+2 X_{a} X_{b}^{3} X_{c}^{2} X_{d}+X_{a}^{2} X_{b}^{3} X_{c}^{2} X_{d} \\
F_{b}= & 1+X_{d}+2 X_{b} X_{d}+X_{b}^{2} X_{d}+X_{b}^{2} X_{c} X_{d}+X_{a} X_{b}^{2} X_{c} X_{d} \\
F_{c}= & 1+X_{d}+3 X_{b} X_{d}+3 X_{b}^{2} X_{d}+3 X_{b}^{2} X_{c} X_{d}+X_{b}^{3} X_{d}+2 X_{b}^{3} X_{c} X_{d}+X_{b}^{3} X_{c}^{2} X_{d} \\
& +3 X_{a} X_{b}^{2} X_{c} X_{d}+3 X_{a} X_{b}^{3} X_{c} X_{d}+3 X_{a} X_{b}^{3} X_{c}^{2} X_{d}+3 X_{a}^{2} X_{b}^{3} X_{c}^{2} X_{d} \\
& +X_{a}^{3} X_{b}^{3} X_{c}^{2} X_{d}
\end{aligned}
$$




$$
\begin{aligned}
F_{d}= & +2 X_{d}+X_{d}^{2}+6 X_{b} X_{d}+6 X_{b} X_{d}^{2}+6 X_{b}^{2} X_{d}+15 X_{b}^{2} X_{d}^{2}+3 X_{b}^{2} X_{c} X_{d}+3 X_{b}^{2} X_{c} X_{d}^{2} \\
& +2 X_{b}^{3} X_{d}+20 X_{b}^{3} X_{d}^{2}+2 X_{b}^{3} X_{c} X_{d}+12 X_{b}^{3} X_{c} X_{d}^{2}+15 X_{b}^{4} X_{d}^{2}+18 X_{b}^{4} X_{c} X_{d}^{2} \\
& +3 X_{b}^{4} X_{c}^{2} X_{d}^{2}+6 X_{b}^{5} X_{d}^{2}+12 X_{b}^{5} X_{c} X_{d}^{2}+6 X_{b}^{5} X_{c}^{2} X_{d}^{2}+X_{b}^{6} X_{d}^{2}+3 X_{b}^{6} X_{c} X_{d}^{2} \\
& +3 X_{b}^{6} X_{c}^{2} X_{d}^{2}+X_{b}^{6} X_{c}^{3} X_{d}^{2}+3 X_{a} X_{b}^{2} X_{c} X_{d}+3 X_{a} X_{b}^{2} X_{c} X_{d}^{2}+3 X_{a} X_{b}^{3} X_{c} X_{d} \\
& +12 X_{a} X_{b}^{3} X_{c} X_{d}^{2}+18 X_{a} X_{b}^{4} X_{c} X_{d}^{2}+6 X_{a} X_{b}^{4} X_{c}^{2} X_{d}^{2}+12 X_{a} X_{b}^{5} X_{c} X_{d}^{2} \\
& +12 X_{a} X_{b}^{5} X_{c}^{2} X_{d}^{2}+3 X_{a} X_{b}^{6} X_{c} X_{d}^{2}+6 X_{a} X_{b}^{6} X_{c}^{2} X_{d}^{2}+3 X_{a} X_{b}^{6} X_{c}^{3} X_{d}^{2}+3 X_{a}^{2} X_{b}^{4} X_{c}^{2} X_{d}^{2} \\
& +6 X_{a}^{2} X_{b}^{5} X_{c}^{2} X_{d}^{2}+3 X_{a}^{2} X_{b}^{6} X_{c}^{2} X_{d}^{2}+3 X_{a}^{2} X_{b}^{6} X_{c}^{3} X_{d}^{2}+X_{a}^{3} X_{b}^{6} X_{c}^{3} X_{d}^{2}
\end{aligned}
$$

The coordinate transformations in the last two cases are written in the form of the factorisation formula (A.17) and are obtained from the corresponding mutation sequences described in Proposition 3.7.

\section{Proof.}

(1) The case where $i \neq j$ follows from the Lie group identity $e_{-j} e_{i}=e_{i} e_{-j}$. The other case follows from the Lie group identity

$$
e_{-i} X_{b}^{\omega_{i}^{\vee}} e_{i}=\left(\frac{X_{b}}{1+X_{b}}\right)^{\omega_{i}^{\vee}}\left(e_{i}\right)\left(\frac{1}{X_{b}}\right)^{\omega_{i}^{\vee}}\left(e_{-i}\right)\left(\frac{X_{b}}{1+X_{b}}\right)^{\omega_{i}^{\vee}} \prod_{j \neq i}\left(1+X_{b}\right)^{-\mathrm{C}_{i j} \omega_{j}^{\vee}} .
$$

(2) The case $\mathrm{C}_{i j}=\mathrm{C}_{j i}=0$ follows from the Lie group identity

$$
e_{j} e_{i}=e_{i} e_{j}
$$

The case $\mathrm{C}_{i j}=\mathrm{C}_{j i}=-1$ follows from the Lie group identity

$$
e_{i} X_{b}^{\omega_{i}^{\vee}} e_{j} e_{i}=\left(1+X_{b}\right)^{\omega_{i}^{\vee}}\left(\frac{X_{b}}{1+X_{b}}\right)^{\omega_{j}^{\vee}}\left(e_{j}\right)\left(\frac{1}{X_{b}}\right)^{\omega_{j}^{\vee}}\left(e_{i} e_{j}\right)\left(1+X_{b}\right)^{\omega_{j}^{\vee}}\left(\frac{X_{b}}{1+X_{b}}\right)^{\omega_{i}^{\vee}} \text {. }
$$

The other two cases can be proved by a computer check and the technique of cluster folding. See [FG06, Section 3.6, 3.7].

\subsection{Cluster $\mathrm{K}_{2}$ Structure on $\operatorname{Conf}_{d}^{b}\left(\mathcal{A}_{\mathrm{sc}}\right)$ and $\operatorname{Conf}_{d}^{b}\left(\mathcal{A}_{\mathrm{sc}}^{\mathrm{fr}}\right)$}

In this section we associate a coordinate chart of the decorated double Bott-Samelson cell Conf ${ }_{d}^{b}\left(\mathcal{A}_{\mathrm{sc}}\right)$ to every seed $\mathbf{s}$ obtained from a triangulation and show that these coordinate charts are related by cluster $\mathrm{K}_{2}$ mutations corresponding to the seed mutations stated in Proposition 3.7, equipping $\operatorname{Conf}_{d}^{b}\left(\mathcal{A}_{\mathrm{sc}}\right)$ with a natural cluster $\mathrm{K}_{2}$ structure. By restricting the cluster $\mathrm{K}_{2}$ structure on $\operatorname{Conf}_{d}^{b}\left(\mathcal{A}_{\mathrm{sc}}\right)$ to the framed double Bott-Samelson cell $\operatorname{Conf}_{d}^{b}\left(\mathcal{A}_{\mathrm{sc}}^{\mathrm{fr}}\right)$, we also obtain a cluster $\mathrm{K}_{2}$ structure on the latter.

Take a point in $\operatorname{Conf}_{d}^{b}\left(\mathcal{A}_{\mathrm{sc}}\right)$. We can propagate the decoration over $\mathrm{B}^{0}$ horizontally across the top of the trapezoid using Lemma 2.10, equipping each flag on the top base with a decoration so that every adjacent pair is compatible. Similarly, we can do the same on the bottom base: propagating the decoration over $\mathrm{B}_{n}$ horizontally across the bottom and equipping each flag on the bottom base with a decoration.

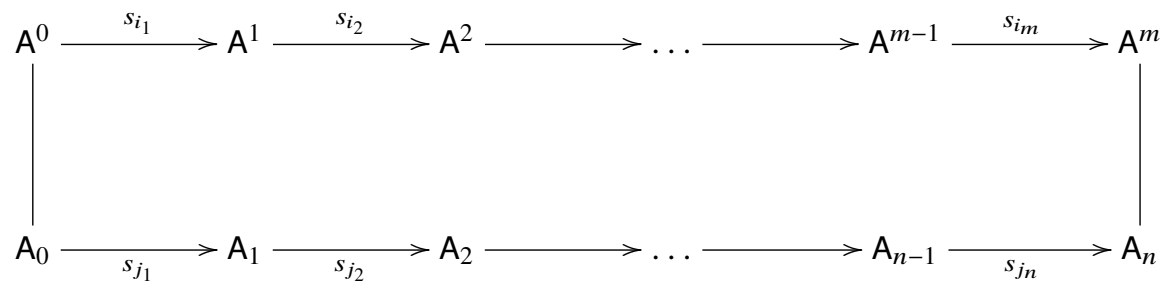


Definition 3.20. Fix a triangulation and consider its corresponding string diagram and seed. Let $a$ be a string on the $i$ th level in the string diagram (and hence a vertex in the corresponding seed). Let $\mathrm{A}_{l} \longrightarrow \mathrm{A}^{k}$ bea diagonal in the triangulation that $a$ intersects. Let us set

$$
A_{a}:=\Delta_{\omega_{i}}\left(\mathrm{~A}_{l}, \mathrm{~A}^{k}\right)
$$

For notation convenience we also adopt the shorthand $\Delta_{i}(l, k)=\Delta_{\omega_{i}}\left(\mathrm{~A}_{l}, \mathrm{~A}^{k}\right)$.

Because a string may cross many different diagonals, the first thing we need to check is the welldefined-ness of $A_{a}$.

Proposition 3.21. The functions $A_{a}$ are well defined.

Proof. It suffices to show that for a string $a$ on the $i$ th level that intersects both sides of a triangle, the functions $A_{a}$ obtained by using each of the two sides of the triangle are equal. Without loss of generality, we suppose that the triangle looks like the following (after fixing a representative), with $t \in \mathrm{T}_{\mathrm{sc}}$ :

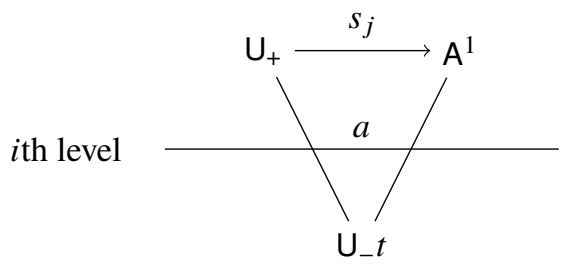

By Lemma A.6, we know that $\mathrm{A}^{1}=e_{j}(p) \bar{s}_{j} \mathrm{U}_{+}$. Then

$$
\begin{aligned}
\Delta_{\omega_{i}}\left(\mathrm{U}_{-} t, \mathrm{~A}^{1}\right) & =\left(t e_{j}(p) \bar{s}_{j}\right)^{\omega_{i}}=\left(t e_{-j}\left(p^{-1}\right) p^{\alpha_{j}^{\vee}} e_{j}\left(-p^{-1}\right)\right)^{\omega_{i}}=\left(t p^{\alpha_{j}^{\vee}}\right)^{\omega_{i}}=t^{\omega_{i}} \\
& =\Delta_{\omega_{i}}\left(\mathrm{U}_{-} t, \mathrm{U}_{+}\right),
\end{aligned}
$$

where $\left(t p^{\alpha_{j}^{\vee}}\right)^{\omega_{i}}=t^{\omega_{i}}$ uses the assumption that $i \neq j$.

Corollary 3.22. For a triangle of either of the following forms
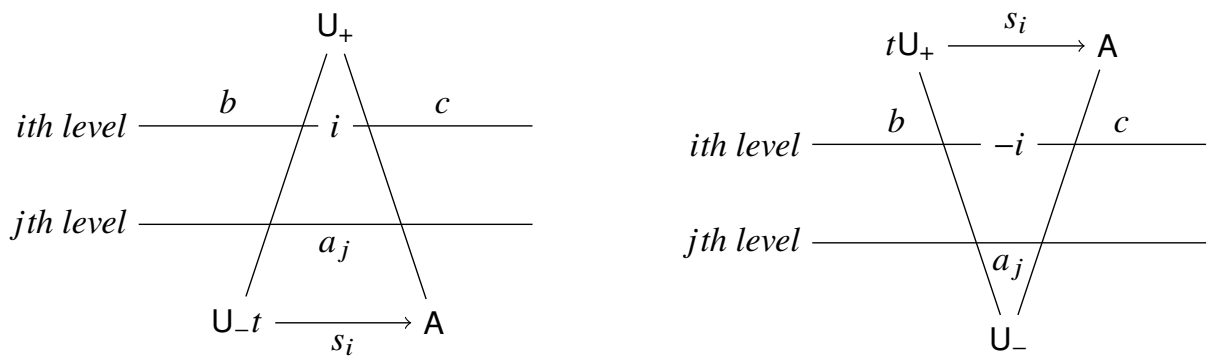

the underlying undecorated flag $\mathrm{B}$ associated to $\mathrm{A}$ is

$$
\mathrm{B}=e_{ \pm i}\left(\frac{\prod_{j \neq i} A_{a_{j}}^{-\mathrm{C}_{j i}}}{A_{b} A_{c}}\right) \mathrm{B}_{ \pm},
$$

where the \pm sign depends on the orientation of the triangle in the same way as the sign of the corresponding node in the string diagram. 
Proof. We will only show the computation for the case on the right; the case on the left is completely analogous. By comparison, we see that we need to act by $t$ to move the triangle in the last proof into the configuration stated in the corollary. Therefore,

$$
\mathrm{B}=t e_{i}(p) \bar{s}_{i} \mathrm{~B}_{+}=e_{i}\left(t^{\alpha_{i}} p\right) \bar{s}_{i} \mathrm{~B}_{+}=e_{-i}\left(t^{-\alpha_{i}} p^{-1}\right) \mathrm{B}_{+} .
$$

But then because $A_{b}=t^{\omega_{i}}, A_{c}=t^{\omega_{i}} p$ and $A_{a_{j}}=t^{\omega_{j}}$ for any $j \neq i$, we have

$$
\frac{\prod_{j \neq i} A_{a_{j}}^{-\mathrm{C}_{j i}}}{A_{b} A_{c}}=\frac{\prod_{j \neq i} t^{-\mathrm{C}_{j i} \omega_{j}}}{p t^{2 \omega_{i}}}=t^{-\sum_{j} \mathrm{C}_{j i} \omega_{j}} p^{-1}=t^{-\alpha_{i}} p^{-1} .
$$

Proposition 3.23. An assignment of $\mathbb{G}_{m}$ values to all of the functions $\left\{A_{a}\right\}$ associated to a triangulation recovers a point in $\operatorname{Conf}_{d}^{b}\left(\mathcal{A}_{\mathrm{sc}}\right)$. As a corollary, these functions form a torus chart on $\operatorname{Conf}_{d}^{b}\left(\mathcal{A}_{\mathrm{sc}}\right)$.

Proof. The idea is to mimic the proof of Lemma 3.10 and use the unipotent elements given by Corollary 3.22 to construct the $b$-chain and the $d$-chain of decorated flags. Without loss of generality, we may assume that the leftmost triangle is of the following shape:

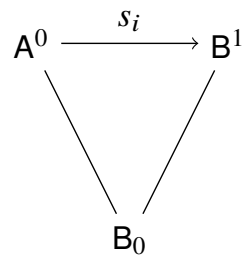

Then we can set $\mathrm{A}_{0}=\mathrm{U}_{-}$and $\mathrm{A}^{0}=t \mathrm{U}_{+}$with $t:=\prod_{j=1}^{\tilde{r}} A_{\left(\begin{array}{l}j \\ 0\end{array}\right)}^{\alpha_{j}^{\vee}}$. Then by Corollary 3.22 we get $\mathrm{B}^{1}=e_{-i}(p) \mathrm{B}_{+}$with $p=\frac{\prod_{j \neq i} A_{\left(\begin{array}{c}j \\ 0\end{array}\right)}^{-C_{j i}}}{A_{\left(\begin{array}{c}i \\ 0\end{array}\right)} A_{\left(\begin{array}{c}i \\ 1\end{array}\right)}}$. Then the compatibility condition requires that the decorated flag $\mathrm{A}^{1}$ is given by $e_{-i}(p) r \mathrm{U}_{+}$with $r:=t\left(t^{\alpha_{i}} p\right)^{-\alpha_{i}^{\vee}}$ as a maximal torus element.

The rest proceeds similar to the proof of Lemma 3.10, with one slight caveat: when the triangles switch orientation (between upward pointing and downward pointing), we need to move the whole configuration by not just a unipotent element but a product of a unipotent element and a maximal torus element so that we may continue to use Corollary 3.22. For example, suppose that the second triangle looks like the picture on the left below. Then we need to move the whole configuration by $r^{-1} e_{-i}(-p)$.

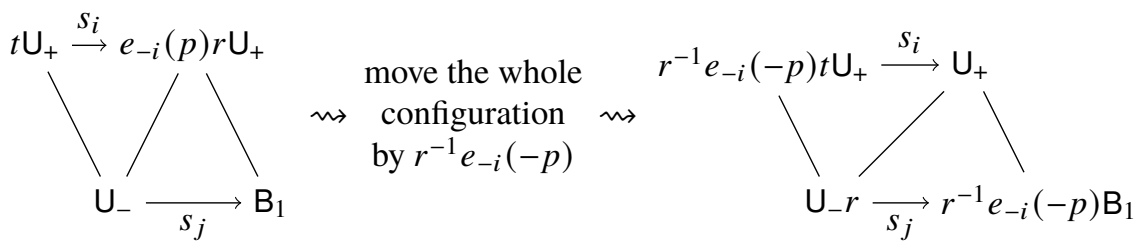

In the end we will get a $b$-chain and a $d$-chain of compatible decorated flags, with their initial flags and terminal flags in general position. Then to get a point in $\operatorname{Conf}_{d}^{b}\left(\mathcal{A}_{\mathrm{sc}}\right)$, we simply forget the decorations everywhere except $A^{0}$ and $A_{n}$.

Definition 3.24. We call the functions $A_{a}$ the cluster $\mathrm{K}_{2}$ coordinates associated to the seed (string diagram/triangulation) on $\operatorname{Conf}_{d}^{b}\left(\mathcal{A}_{\mathrm{sc}}\right)$. 
Because the framed double Bott-Samelson cell $\operatorname{Conf}_{d}^{b}\left(\mathcal{A}_{\mathrm{sc}}^{\mathrm{fr}}\right)$ has compatible decorated flags around the perimeter, it can be seen as the subset of $\operatorname{Conf}_{d}^{b}\left(\mathcal{A}_{\mathrm{sc}}\right)$ with all cluster $\mathrm{K}_{2}$ coordinates associated to open strings set to 1 . It then follows that the cluster $\mathrm{K}_{2}$ coordinates associated to closed strings restrict to a set of coordinates on $\operatorname{Conf}_{d}^{b}\left(\mathcal{A}_{\mathrm{sc}}^{\mathrm{fr}}\right)$, which we call the cluster $\mathrm{K}_{2}$ coordinates on $\operatorname{Conf}_{d}^{b}\left(\mathcal{A}_{\mathrm{sc}}^{\mathrm{fr}}\right)$ associated to the seed (string diagram/triangulation).

In order to justify the name 'cluster $\mathrm{K}_{2}$ coordinates', we need to show that they actually transform as cluster $\mathrm{K}_{2}$ coordinates.

\section{Proposition 3.25.}

(1) If $i \neq j$, then

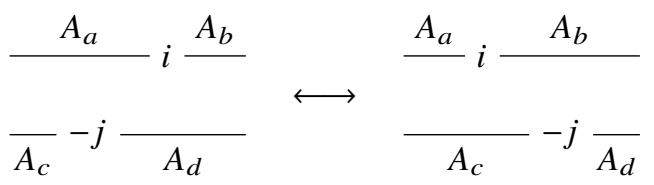

On the other hand, if we interchange two neighbouring nodes of opposite signs on the same level, we get

$$
\text { ith level } \frac{A_{a}}{j \text { th level }-i \frac{A_{b}}{A_{j}} i \frac{A_{c}}{A_{j}}} \longleftrightarrow \quad \text { ith level } \frac{A_{a}}{j \text { th level } \frac{A_{b}^{\prime}}{A_{j}}-i \frac{A_{c}}{}}
$$

where

$$
A_{b}^{\prime}=\frac{1}{A_{b}}\left(A_{a} A_{c}+\prod_{j \neq i} A_{j}^{-\mathrm{C}_{j i}}\right) .
$$

(2) If we perform a braid move to the string diagram, depending on whether it is of Dynkin type $\mathrm{A}_{1} \times \mathrm{A}_{1}$, $\mathrm{A}_{2}, \mathrm{~B}_{2}$ or $\mathrm{G}_{2}$, the cluster Poisson coordinates transform according to the sequences of mutations described in Proposition 3.7 (we will only depict the case where a braid move takes place among nodes labelled by simple roots; the cases where they are labelled by opposite simple roots are completely analogous).

○ $\mathrm{C}_{i j} \mathrm{C}_{j i}=0$ :

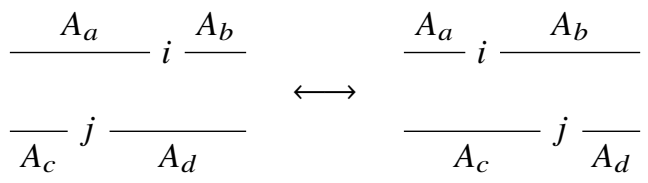

$\circ \mathrm{C}_{i j} \mathrm{C}_{j i}=-1$ :

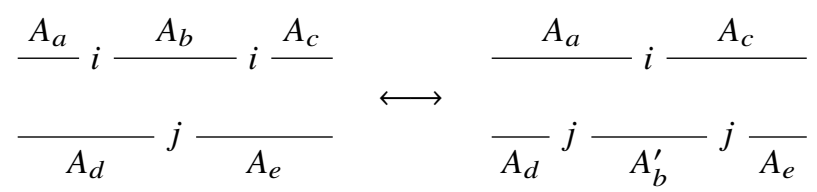


where

$$
A_{b}^{\prime}=\frac{1}{A_{b}}\left(A_{a} A_{e}+A_{c} A_{d}\right)
$$

$\circ \mathrm{C}_{i j}=-2$ and $\mathrm{C}_{j i}=-1$ :

$$
\begin{aligned}
& \frac{A_{c}}{2} i \frac{A_{a}}{A_{d}} \quad \frac{A_{c}}{\longleftarrow} i \frac{A_{a}^{\prime}}{i} \frac{A_{d}}{2} \\
& \frac{}{A_{e}} j \frac{}{A_{b}} j \frac{}{A_{f}} \longleftrightarrow \frac{}{A_{e}} j \frac{}{A_{b}^{\prime}} j \frac{}{A_{f}}
\end{aligned}
$$

where

$$
A_{a}^{\prime}=\frac{A_{a} A_{f}}{A_{b}} \tilde{F}_{a}, \quad \text { and } \quad A_{b}^{\prime}=\frac{A_{c} A_{f}}{A_{b}} \tilde{F}_{b}
$$

and $\tilde{F}_{a}$ and $\tilde{F}_{b}$ are the polynomials in Equation (3.18) with the substitution $X_{k}=\prod_{l} A_{l}^{\epsilon_{k l}}$ where $\epsilon$ is the exchange matrix associated to the seed on the left.

$\circ \mathrm{C}_{i j}=-3$ and $\mathrm{C}_{j i}=-1$ :

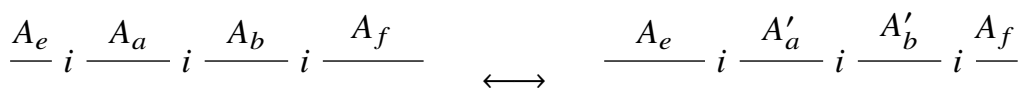

$$
\begin{aligned}
& \overline{A_{g}} j \frac{}{A_{c}} j \frac{}{A_{d}} j \overline{A_{h}} \quad \longleftrightarrow \quad \frac{}{A_{g}} j \frac{}{A_{c}^{\prime}} j \frac{}{A_{d}^{\prime}} j \frac{}{A_{h}}
\end{aligned}
$$

where

$$
A_{a}^{\prime}=\frac{A_{a} A_{h}}{A_{d}} \tilde{F}_{a} \quad A_{b}^{\prime}=\frac{A_{b} A_{h}}{A_{d}} \tilde{F}_{b} \quad A_{c}^{\prime}=\frac{A_{g} A_{h}}{A_{d}} \tilde{F}_{c} \quad A_{d}^{\prime}=\frac{A_{c} A_{h}^{2}}{A_{d}^{-2}} \tilde{F}_{d}
$$

and $\tilde{F}_{a}, \tilde{F}_{b}, \tilde{F}_{c}, \tilde{F}_{d}$ are the polynomials in Equation (3.19) with the substitution $X_{k}=\prod_{l} A_{l}^{\epsilon_{k l}}$ where $\epsilon$ is the exchange matrix associated to the seed on the left.

The coordinate transformations in the last two cases are written in the form of the factorisation formula (A.16).

\section{Proof.}

(1) The case where $i \neq j$ is clear from the definition of cluster $\mathrm{K}_{2}$ coordinates. For the other case, we can use Corollary 3.22 to recover the local configuration of decorated flags.

$$
\begin{aligned}
& e_{-i}\left(-\frac{\prod_{j \neq i} A_{j}^{-C_{j i}}}{A_{a} A_{b}}\right) A_{a}^{\alpha_{i}^{\vee}} \prod_{j \neq i} A_{j}^{\alpha_{j}^{\vee}} \mathrm{U}_{+} \stackrel{s_{i}}{\longrightarrow} A_{b}^{\alpha_{i}^{\vee}} \prod_{j \neq i} A_{j}^{\alpha_{j}^{\vee}} \mathrm{U}_{+} \\
& \mathrm{U}_{-} \underset{s_{i}}{\longrightarrow} \mathrm{U}_{-} A_{c}^{\alpha_{i}^{\vee}} \prod_{j \neq i} A_{j}^{\alpha_{j}^{\vee}} e_{i}\left(-\frac{\prod_{j \neq i} A_{j}^{-\mathrm{C}_{j i}}}{A_{b} A_{c}}\right) A_{b}^{-\alpha_{i}^{\vee}} \prod_{j \neq i} A_{j}^{-\alpha_{j}^{\vee}}
\end{aligned}
$$


By using the Lie group identity $e_{i}(q) t=t e_{i}\left(t^{-\alpha_{i}} q\right)$, we can simplify the bottom right decorated flag as $U_{-}\left(\frac{A_{c}}{A_{b}}\right)^{\alpha_{i}^{\vee}} e_{i}\left(-\frac{A_{b}}{A_{c}}\right)$. Now we want to flip the diagonal and compute $\Delta_{\omega_{i}}$ along this new diagonal. Note that

$$
\begin{aligned}
& \left(\frac{A_{c}}{A_{b}}\right)^{\alpha_{i}^{\vee}} e_{i}\left(-\frac{A_{b}}{A_{c}}\right) e_{-i}\left(-\frac{\prod_{j \neq i} A_{j}^{-\mathrm{C}_{j i}}}{A_{a} A_{b}}\right) A_{a}^{\alpha_{i}^{\vee}} \prod_{j \neq i} A_{j}^{\alpha_{j}^{\vee}} \\
& =\left(\frac{A_{c}}{A_{b}}\right)^{\alpha_{i}^{\vee}} e_{-i}(\cdots)\left(1+\frac{\prod_{j \neq i} A_{j}^{-\mathrm{C}_{j i}}}{A_{a} A_{c}}\right)^{\alpha_{i}^{\vee}} e_{i}(\cdots) A_{a}^{\alpha_{i}^{\vee}} \prod_{j \neq i} A_{j}^{\alpha_{j}^{\vee}} \\
& =e_{-i}(\cdots)\left(\frac{1}{A_{b}}\left(A_{a} A_{c}+\prod_{j \neq i} A_{j}^{-\mathrm{C}_{j i}}\right)\right)^{\alpha_{i}^{\vee}} \prod_{j \neq i} A_{j}^{\alpha_{j}^{\vee}} e_{i}(\cdots) .
\end{aligned}
$$

Therefore, it follows that $A_{b}^{\prime}=\frac{1}{A_{b}}\left(A_{a} A_{c}+\prod_{j \neq i} A_{j}^{-\mathrm{C}_{j i}}\right)$.

(2) The case $\mathrm{C}_{i j}=\mathrm{C}_{j i}=0$ is clear from the definition of cluster $\mathrm{K}_{2}$ coordinates. For the case $\mathrm{C}_{i j}=\mathrm{C}_{j i}=-1$, we again use the computation in the proof of Proposition 3.21 to recover the local configuration of decorated flags from the string diagram on the left as follows (to save space we write the diagram sideways):

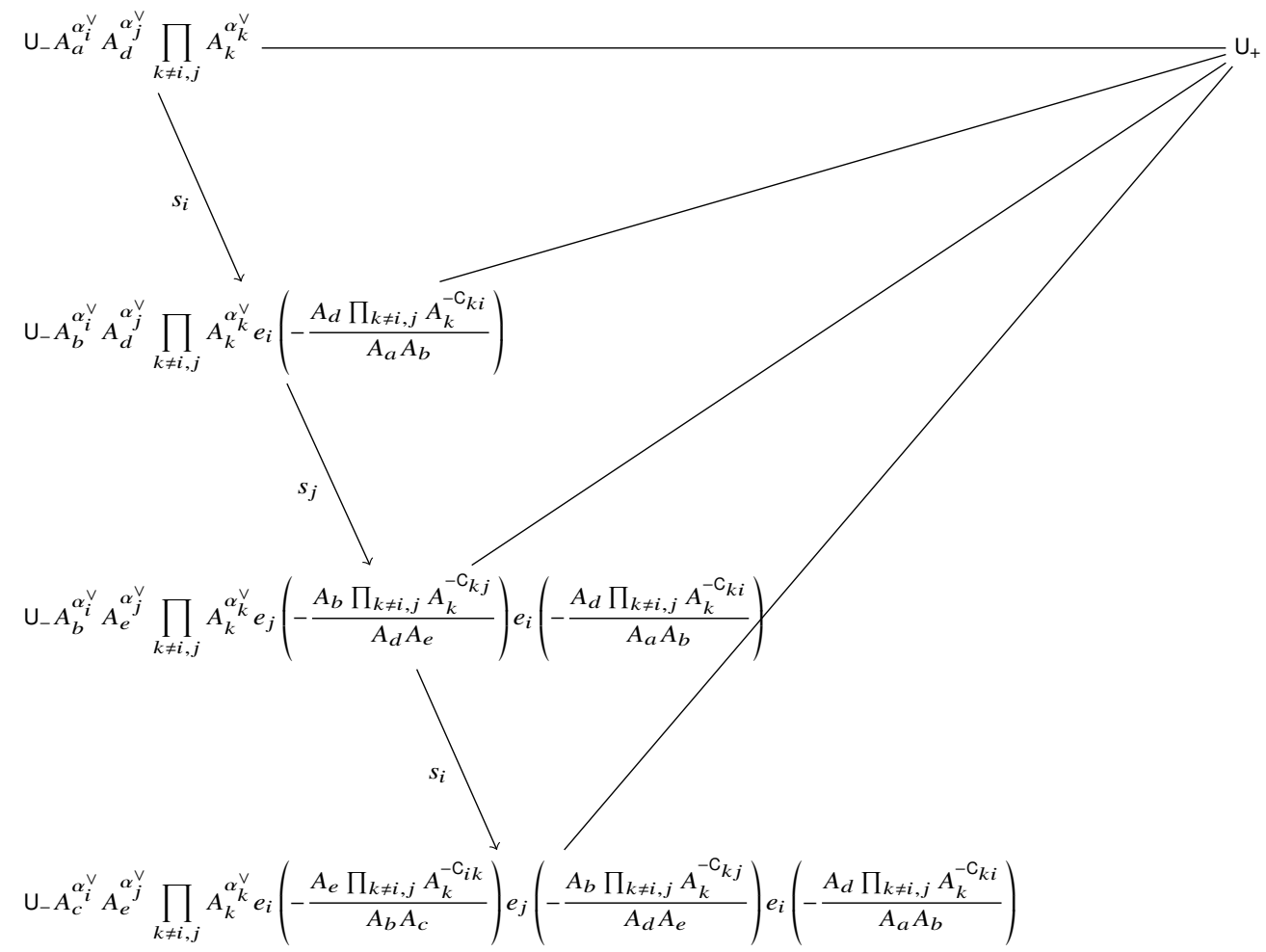

Similarly, from the string diagram on the right we have the following configuration of decorated flags: 


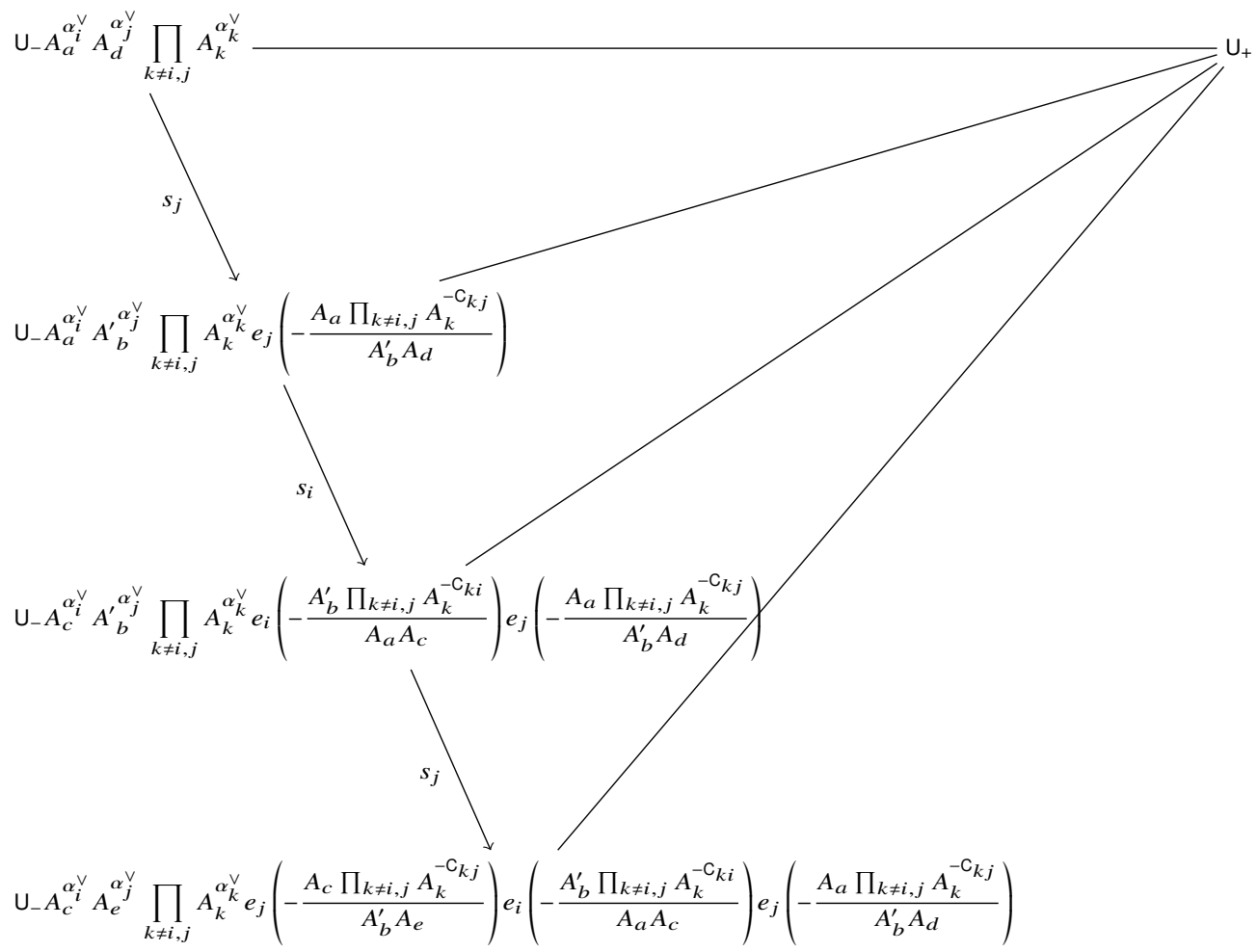

By the uniqueness part of Lemma 2.11, it suffices to show that the last decorated flags from the two diagrams are equal, which boils down to showing that

$$
\begin{aligned}
& e_{i}\left(-\frac{A_{e} \prod_{k \neq i, j} A_{k}^{-\mathrm{C}_{k i}}}{A_{b} A_{c}}\right) e_{j}\left(-\frac{A_{b} \prod_{k \neq i, j} A_{k}^{-\mathrm{C}_{k j}}}{A_{d} A_{e}}\right) e_{i}\left(-\frac{A_{d} \prod_{k \neq i, j} A_{k}^{-\mathrm{C}_{k i}}}{A_{a} A_{b}}\right) \\
= & e_{j}\left(-\frac{A_{c} \prod_{k \neq i, j} A_{k}^{-\mathrm{C}_{k j}}}{A_{b}^{\prime} A_{e}}\right) e_{i}\left(-\frac{A_{b}^{\prime} \prod_{k \neq i, j} A_{k}^{-\mathrm{C}_{k i}}}{A_{a} A_{c}}\right) e_{j}\left(-\frac{A_{a} \prod_{k \neq i, j} A_{k}^{-\mathrm{C}_{k j}}}{A_{b}^{\prime} A_{d}}\right) .
\end{aligned}
$$

But this is precisely the Lie group identity

$$
e_{i}\left(q_{1}\right) e_{j}\left(q_{2}\right) e_{i}\left(q_{3}\right)=e_{j}\left(\frac{q_{2} q_{3}}{q_{1}+q_{3}}\right) e_{i}\left(q_{1}+q_{3}\right) e_{j}\left(\frac{q_{1} q_{2}}{q_{1}+q_{3}}\right) .
$$

The proof for the other two cases can be found in [GS19, Section 7.6] here

From the proof of case 1 above we also deduce the following identity, which is useful in the next section.

Corollary 3.26. Suppose that $\mathrm{G}=\mathrm{G}_{\mathrm{sc}}$ and $\mathrm{A}^{k} \stackrel{s_{i}}{\longrightarrow} \mathrm{A}^{k+1}$ and $\mathrm{A}_{l} \stackrel{s_{i}}{\longrightarrow} \mathrm{A}_{l+1}$ are compatible pairs that are part of a decorated double Bott-Samelson cell. Then

$$
\Delta_{j}(l+1, k) \Delta_{i}(l, k+1)-\Delta_{i}(l, k) \Delta_{i}(l+1, k+1)=\prod_{j \neq i} \Delta_{j}(*, *)^{-C_{j i}},
$$

where $(*, *)$ means either of $(l, k),(l, k+1),(l+1, k)$ and $(l+1, k+1)$. 
Proof. First we observe that both sides of the equation are regular functions on the decorated double Bott-Samelson cell and therefore it suffices to show the equality with four extra open conditions:

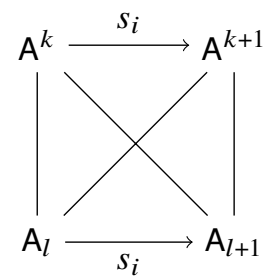

But then this reduces to the identity

$$
A_{b}^{\prime} A_{b}-A_{a} A_{c}=\prod_{j \neq i} A_{j}^{-\mathrm{C}_{j i}}
$$

in case 1 of the last proposition.

Lastly, let us investigate how the cluster $\mathrm{K}_{2}$ coordinates transform under the transposition morphism.

Proposition 3.27. Let $\left(A_{a}\right)$ and $\left(A_{a}^{\prime}\right)$ be the cluster $\mathrm{K}_{2}$ coordinate charts associated to two corresponding string diagrams under transposition. Then under the transposition morphism $t: \operatorname{Conf}_{d}^{b}\left(\mathcal{A}_{\mathrm{sc}}\right) \rightarrow$ $\operatorname{Conf}_{b^{\circ}}^{d^{\circ}}\left(\mathcal{A}_{\mathrm{ad}}\right), t^{*} A_{a}^{\prime}=A_{a}$.

Proof. It follows from Lemma 2.6.

\subsection{Coordinate Rings as Upper Cluster Algebras}

Decorated Bott-Samelson cells are affine. In this section we show that their coordinate rings are

$$
\mathcal{O}\left(\operatorname{Conf}_{d}^{b}\left(\mathcal{A}_{\mathrm{sc}}\right)\right) \cong \operatorname{up}\left(\mathscr{A}_{d}^{b}\right) \quad \text { and } \quad \mathcal{O}\left(\operatorname{Conf}_{d}^{b}\left(\mathcal{A}_{\mathrm{ad}}\right)\right) \cong \operatorname{up}\left(\mathscr{X}_{d}^{b}\right)
$$

where up $\left(\mathscr{A}_{d}^{b}\right)$ is the upper cluster algebra arising from the family of mutation equivalent seeds associated to $(b, d)$ and up $\left(x_{d}^{b}\right)$ is the corresponding cluster Poisson algebra.

Let us start with the first claim. We simplify the notation and write $\mathcal{O}\left(\operatorname{Conf}_{d}^{b}\left(\mathcal{A}_{\mathrm{sc}}\right)\right)$ as $\mathcal{O}_{d}^{b}$.

Lemma 3.28. The ring $\mathcal{O}_{d}^{b}$ is a unique factorisation domain (UFD).

Proof. The proof of Theorem 2.30 shows that $\mathcal{O}_{d}^{b}$ is the nonvanishing locus of some function on $T \times \mathbb{A}^{n}$, which is also the nonvanishing locus of some function on $\mathbb{A}^{N}$ for $N=n+\operatorname{dim} \mathrm{T}$. Note that $\mathcal{O}\left(\mathbb{A}^{N}\right)$ is a polynomial ring. The ring $\mathcal{O}_{d}^{b}$ is a localisation of $\mathcal{O}\left(\mathbb{A}^{N}\right)$ and therefore is a UFD.

Lemma 3.29. The vanishing locus $\left\{A_{c}=0\right\}$ in $\operatorname{Conf}_{d}^{b}\left(\mathcal{A}_{\mathrm{sc}}\right)$ associated with any closed string $c$ is of codimension 1 .

Proof. Fix a triangulation containing $c$ as a closed string. Suppose that $c$ is on the $i$ th level. We assign a Tits codistance $s_{i}$ to a diagonal in the triangulation if this diagonal intersects $c$ and assign a Tits codistance $e$ otherwise. Because $c$ is a closed string, the leftmost and the rightmost diagonals (the two slanted sides of the trapezoid) must be assigned with Tits codistance $e$. Therefore, this configuration describes a subset $W_{c}$ of $\operatorname{Conf}_{d}^{b}\left(\mathcal{A}_{\mathrm{sc}}\right)$. We claim that $W_{c}$ is of codimension 1 within $\operatorname{Conf}_{d}^{b}\left(\mathcal{A}_{\mathrm{sc}}\right)$ and $W_{c} \subset\left\{A_{c}=0\right\}$. Note that the lemma follows from this claim.

Let us compute the dimension of $W_{c}$. Without loss of generality, we can fix the leftmost pair of flags $A^{0}-B_{0}$ for any point in $W_{c}$ to be $U_{+} \longrightarrow B$, which exhausts the diagonal G-action on the 
configuration of flags. By Proposition A.8, we see that for most triangles in the triangulation, under the Tits codistance assignment, each of them contributes a $\mathbb{G}_{m}$ factor of $W_{c}$; the only exceptions are the two triangles containing the endpoints of $c$ and they contribute a $\{*\}$-factor and a $\mathbb{A}^{1}$-factor, respectively. In the end, there is also another decoration over the last flag of the bottom chain, which gives rise to a $\operatorname{dim} \mathrm{T}$-factor of $W_{c}$. In conclusion, this shows that

$$
W_{c} \cong \mathbb{G}_{m}^{l(b)+l(d)-2} \times \mathbb{A}^{1} \times \mathrm{T},
$$

where $l(b)$ and $l(d)$ are the lengths of the positive braids $b$ and $d$. On the other hand, we recall from Theorem 2.30 that

$$
\operatorname{dim} \operatorname{Conf}_{d}^{b}\left(\mathcal{A}_{\mathrm{sc}}\right)=\operatorname{dim} \mathrm{T}+l(b)+l(d) .
$$

Therefore, $W_{c}$ is indeed of codimension 1 .

To see that $W_{c} \subset\left\{A_{c}=0\right\}$, consider the triangle containing the left endpoint of the closed string $c$. Suppose that the triangle is of the following form:

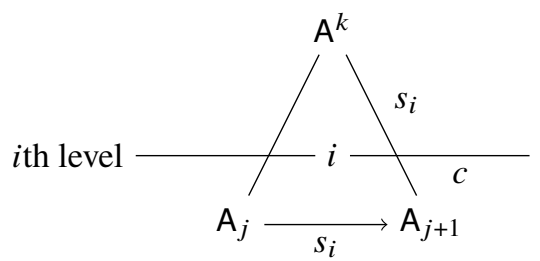

By using the G-action, we may assume without loss of generality that $A^{k}=t U_{+}$and $A_{j}=U_{-}$. Then $\mathrm{A}_{j+1}$ must be $\mathrm{U}_{-} \overline{\bar{s}}_{i}$. Therefore,

$$
A_{c}=\Delta_{\omega_{i}}\left(\overline{\bar{s}}_{i}\right)=0
$$

This shows that $W_{c} \subset\left\{A_{c}=0\right\}$. The case with an upside-down triangle can be proved in a similar way.

Fix a triangulation for $\operatorname{Conf}_{d}^{b}\left(\mathcal{A}_{\mathrm{sc}}\right)$. Take the very first triangle on the left and consider the corresponding node in the string diagram; let $a$ be the closed string on the right of this node and suppose that $a$ is on the $i$ th level. Its associated cluster variable $A_{a}$ is a regular function on $\operatorname{Conf}_{d}^{b}\left(\mathcal{A}_{\mathrm{sc}}\right)$.
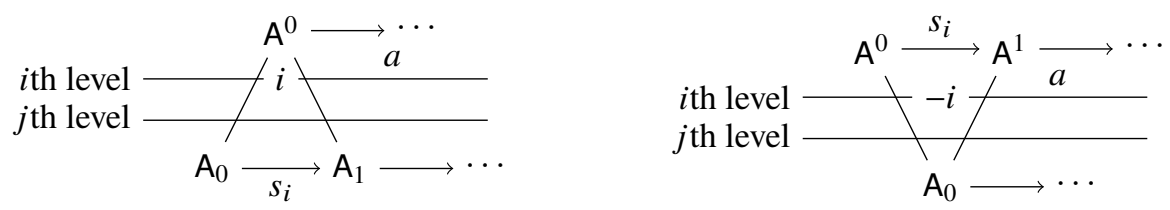

Lemma 3.30. The function $A_{a}$ is an irreducible element of $\mathcal{O}_{d}^{b}$.

Proof. Let us prove the case of the picture on the right; the case of the picture on the left is completely analogous. Without loss of generality, we set $\mathrm{A}^{0}=t \mathrm{U}_{+}$and $\mathrm{A}_{0}=\mathrm{U}_{-}$. From Corollary 3.22 we know that if we impose the general position condition $B^{1}-B_{-}$, then

$$
\mathrm{B}^{1}=e_{-i}\left(\frac{\prod_{j \neq i} t^{-\mathrm{C}_{j i} \omega_{j}}}{t^{\omega_{i}} A_{a}}\right) \mathrm{B}_{+}=e_{i}\left(\frac{t^{\omega_{i}} A_{a}}{\prod_{j \neq i} t^{-\mathrm{C}_{j i} \omega_{j}}}\right) \bar{s}_{i} \mathrm{~B}_{+} .
$$


On the other hand, we know from Lemma A.6 that the argument $\frac{t^{\omega_{i}} A_{a}}{\prod_{j \neq i} t^{-C_{j i} \omega_{j}}}$ parametrises the moduli space of all of the flags that are of Tits distance $s_{i}$ away from $\mathrm{B}_{+}$, which is isomorphic to $\mathbb{A}^{1}$. Because $t^{\omega_{i}}$ and $t^{\omega_{j}}$ (the frozen cluster $\mathrm{K}_{2}$ variables) are never zero on $\operatorname{Conf}_{d}^{b}\left(\mathcal{A}_{\mathrm{sc}}\right)$ by definition, it follows that $A_{a}$ also parametrises the $\mathbb{A}^{1}$ moduli space of flags. Recall from the proof of Theorem 2.30 that such a parameter is precisely one of the generators of the polynomial ring $\mathcal{O}\left(\mathbb{A}^{N}\right)$. Hence, $A_{a}$ is a unit or an irreducible element in $\mathcal{O}_{d}^{b}$. However, $A_{a}$ cannot be a unit, because by Lemma 3.29, its vanishing locus $\left\{A_{a}=0\right\}$ is nonempty and of codimension 1 . Therefore, $A_{a}$ must be an irreducible element.

Next we will use an induction to prove that all nonfrozen cluster $\mathrm{K}_{2}$ coordinates associated to the fixed triangulation are irreducible elements of $\mathcal{O}_{d}^{b}$. Because triangles in a triangulation possess a well-defined ordering from left to right, by associating each closed string $c$ to the triangle corresponding to its left node, we get an ordering < on the closed strings. We will perform an induction according to this order.

Let $c$ be a closed string on the $j$ th level. Let $b^{\prime}$ be the positive braid that is the remaining part of the word for $b$ after deleting all of the letters occurring before the triangle corresponding to the left node of the closed string $c$ and let $d^{\prime}$ be the positive braid that is the remaining part of the word for $d$ after deleting all of the letters occurring before the triangle corresponding to the left node of the closed string $c$.

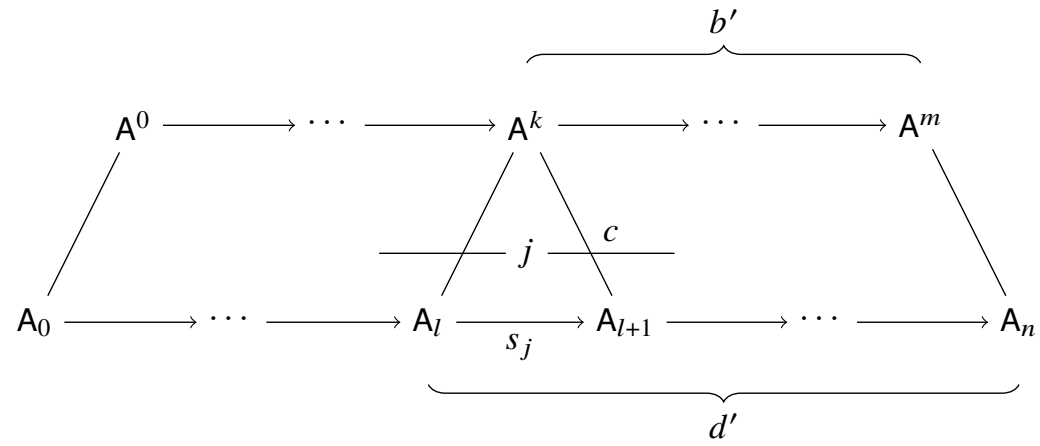

Lemma 3.31. Let $S$ be the set of strings that crosses the diagonal $\mathrm{A}_{l} \longrightarrow \mathrm{A}^{k}$. Then as algebras,

$$
\mathcal{O}_{d}^{b}\left[\frac{1}{\prod_{e<c} A_{e}}\right] \cong \mathbb{C}\left[A_{e}^{ \pm}\right]_{e<c} \underset{\mathbb{C}\left[A_{f}^{ \pm}\right]_{f \in S}}{\otimes} \mathcal{O}_{d^{\prime}}^{b^{\prime}}
$$

Proof. The statement of the proposition is equivalent to the geometric statement that the distinguished open subset (nonvanishing locus) $U_{\prod_{e<c}} A_{e}$ of $\operatorname{Conf}_{d}^{b}\left(\mathcal{A}_{\mathrm{sc}}\right)$ is biregularly isomorphic to a fibre product $T_{<c} \underset{T_{S}}{\times} \operatorname{Conf}_{d^{\prime}}^{b^{\prime}}\left(\mathcal{A}_{\mathrm{sc}}\right)$ where $T_{<c}$ is a torus with coordinates $\left\{A_{e}\right\}_{e<c}$ and $T_{S}$ is a torus with coordinates $\left\{A_{f}\right\}_{f \in S}$. Note that given a point in $U_{\prod_{e<c} A_{e}}$ we automatically get a point in $T_{<c}$ using the coordinates $\left\{A_{e}\right\}_{e<c}$ and a point in $\operatorname{Conf}_{d^{\prime}}^{b^{\prime}}\left(\mathcal{A}_{\mathrm{sc}}\right)$ by taking the decorated flags in the truncated part corresponding to the shape $\left(b^{\prime}, d^{\prime}\right)$ and they are mapped to the same point in the torus $T_{S}$ because they both have $\left\{A_{f}\right\}$ as nonzero coordinate functions. On the other hand, given a point in the fibre product $T_{<c} \times \operatorname{Conf}_{T_{S}}^{b^{\prime}}\left(\mathcal{A}_{\mathrm{sc}}\right)$ we can recover a point in $U_{\prod_{e<c} A_{e}}$ by building some extra decorated flags upon the configuration in $\operatorname{Conf}_{d^{\prime}}^{b^{\prime}}\left(\mathcal{A}_{\mathrm{sc}}\right)$ using the nonzero functions $\left\{A_{e}\right\}_{e<c}$. These two morphisms are obviously regular and inverses of each other; therefore, $U_{\prod_{e<c}} A_{e} \cong T_{<c} \underset{T_{S}}{\times} \operatorname{Conf}_{d^{\prime}}^{b^{\prime}}\left(\mathcal{A}_{\mathrm{sc}}\right)$ and the original statement follows immediately.

Now we are ready to give the proof of the general statement.

Proposition 3.32. $A_{c}$ are irreducible elements in $\mathcal{O}_{d}^{b}$ for all closed strings $c$. 
Proof. We will do an induction based on the order < on closed strings. Lemma 3.30 takes care of the base case. Now inductively suppose that $A_{e}$ are irreducible for all closed strings $e<c$. By Lemma 3.30 we know that $A_{c}$ is an irreducible element of $\mathcal{O}_{d^{\prime}}^{b^{\prime}}$. Then $A_{c}=1 \otimes A_{c}$ is also an irreducible element in $\mathbb{C}\left[A_{e}^{ \pm}\right]_{e<c} \underset{\mathbb{C}\left[A_{f}^{ \pm}\right]_{f \in S}}{\otimes} \mathcal{O}_{d^{\prime}}^{b^{\prime}} \cong \mathcal{O}_{d}^{b}\left[\frac{1}{\prod_{e<c} A_{e}}\right]$ (which is a UFD as well because it is a localisation of a UFD). This means that the factorisation of $A_{c}$ in $\mathcal{O}_{d}^{b}$ must be of the form

$$
A_{c}=F \prod_{e<c} A_{e}^{n_{e}}
$$

for some irreducible element $F$. Now to prove that $A_{c}$ is indeed irreducible in $\mathcal{O}_{d}^{b}$, it suffices to prove that $n_{e}=0$ for all $e<c$.

For each $e<c$, consider the subset $W_{e}$ associated with the closed string $e$ as constructed in the proof of Lemma 3.29. We claim that $W_{e} \subset\left\{A_{e}=0\right.$ but $\left.A_{c} \neq 0\right\}$. If the left endpoint of $c$ lies on the right of the right endpoint of $e$, then the claim is obvious because any diagonal crossing $c$ is assigned with a general position condition. On the other hand, if the left endpoint of $c$ lies on the left of the right endpoint of $e$, we consider the triangle containing the left endpoint of $e$. Note that in this case, the level of $c$ must be distinct from the level of $e$. By symmetry, let us assume that it looks like the following:

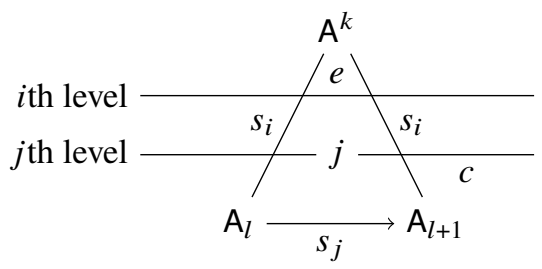

By using the G-action, we can fix $\mathrm{A}^{k}=\bar{s}_{i} t \mathrm{U}_{+}$for some $t \in \mathrm{T}, \mathrm{A}_{l}=\mathrm{U}_{-}$and $\mathrm{A}_{l+1}=\mathrm{U}_{-} \overline{\bar{s}}_{j} e_{-j}(q)$ for some $q \neq 0$. Because $i \neq j$, we have

$$
A_{c}=\Delta_{\omega_{j}}\left(\overline{\bar{s}}_{j} e_{-j} \bar{s}_{i} t \mathrm{U}_{+}\right)=\Delta_{\omega_{j}}\left(q^{\alpha_{j}^{\vee}}\left(s_{i}(t)\right) \bar{s}_{i}\right) \neq 0 .
$$

This shows that $W_{e} \subset\left\{A_{e}=0\right.$ but $\left.A_{c} \neq 0\right\}$, which implies that $\left\{A_{e}=0\right.$ but $\left.A_{c} \neq 0\right\}$ is nonempty. Therefore, we can conclude that $n_{e}=0$ in (3.33).

Now because $A_{c}$ associated to closed strings are all irreducible elements in $\mathcal{O}_{d}^{b}$, their vanishing loci $D_{c}:=\left\{A_{c}=0\right\}$ are all irreducible divisors. Furthermore, we deduce the following corollary from the proposition above.

Corollary 3.34. For any two distinct closed strings $c$ and $e, \operatorname{codim}\left(D_{c} \cap D_{e}\right) \geq 2$ in $\operatorname{Conf}_{d}^{b}\left(\mathcal{A}_{\mathrm{sc}}\right)$ and $\operatorname{ord}_{D_{c}} A_{e}=0$.

Proof. First note that the codimension statement follows from the order statement. This is because $\operatorname{ord}_{D_{c}} A_{e}=0$ implies that $A_{e}$ is invertible along $D_{c}$ and hence $D_{c} \backslash D_{e}$ is open in $D_{c}$; but then because $D_{c}$ and $D_{e}$ are both irreducible, this is equivalent to codim $\left(D_{c} \cap D_{e}\right) \geq 2$.

To compute the order of $A_{e}$ along $D_{c}$, note that $\operatorname{ord}_{D_{c}} A_{e} \geq 0$ because $A_{e}$ is a regular function on $\operatorname{Conf}_{d}^{b}\left(\mathcal{A}_{\mathrm{sc}}\right)$. On the other hand, $\operatorname{ord}_{D_{c}} A_{e}>0$ implies that $A_{e}$ is a multiple of $A_{c}$, which is impossible, as we saw from the proof of last proposition.

Our strategy to show that $\mathcal{O}_{d}^{b} \cong$ up $\left(\mathscr{A}_{d}^{b}\right)$ is analogous to Berenstein, Fomin and Zelevinsky's proof that the coordinate ring of double Bruhat cells is an upper cluster algebra, which relies on the following theorem.

Theorem 3.35 ([BFZ05], Corollary 1.9). Fix an initial seed $\mathbf{s}_{0}$ and let $\mathbf{A}_{0}:=\left\{A_{a}\right\}$ be the corresponding initial $\mathrm{K}_{2}$ cluster. Let $\mathbf{A}_{c}$ be the $\mathrm{K}_{2}$ cluster obtained from $\mathbf{A}$ via a single mutation in the direction of $c$. 
If the restriction of the exchange matrix $\left.\epsilon_{; \mathbf{s}_{0}}\right|_{I^{u+} \times I}$ is full-ranked, then upper cluster algebra up $(\mathcal{A})$ is equal to the intersection

$$
\mathbb{C}\left[\mathbf{A}_{0}^{ \pm}\right] \cap \bigcap_{\text {c nonfrozen }} \mathbb{C}\left[\mathbf{A}_{c}^{ \pm}\right] \subset \operatorname{Frac}\left(\mathbb{C}\left[\mathbf{A}_{0}\right]\right) .
$$

In geometric terms, the full-rank condition on $\left.\epsilon_{; \mathbf{s}_{0}}\right|_{I^{\text {uf }} \times I}$ is equivalent to saying that the canonical map $p: T_{\mathscr{A} ; \mathbf{s}_{0}} \rightarrow T_{\mathscr{X} \text { uf } ; \mathbf{s}_{0}^{\text {uf }}}$ is surjective (see Appendix A.2 for definition) and the intersection of Laurent polynomial rings is precisely the coordinate ring of the union of the corresponding seed tori Spec $\mathbb{C}\left[\mathbf{A}_{0}^{ \pm}\right] \cup \cup_{c}$ Spec $\mathbb{C}\left[\mathbf{A}_{c}^{ \pm}\right]$.

Going back to the decorated double Bott-Samelson cell Conf ${ }_{d}^{b}\left(\mathcal{A}_{\mathrm{sc}}\right)$, because we are free to choose any seed in the mutation equivalent family as the initial seed, for the sake of simplicity let us fix our initial seed to be one that is associated to a triangulation in which all triangles of the form

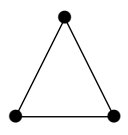
come before triangles of the form ; that is, a triangulation that looks like the following:

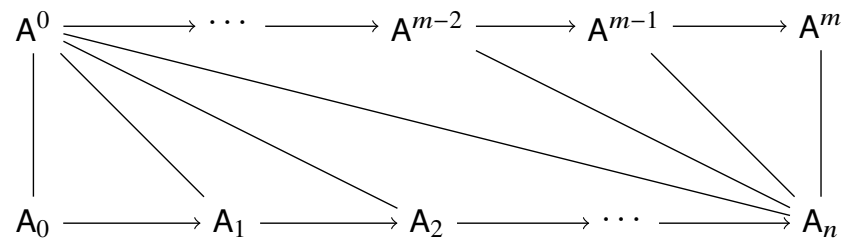

Note that any closed string $c$ in the corresponding string diagram lies in a part that is of one of the following three forms:
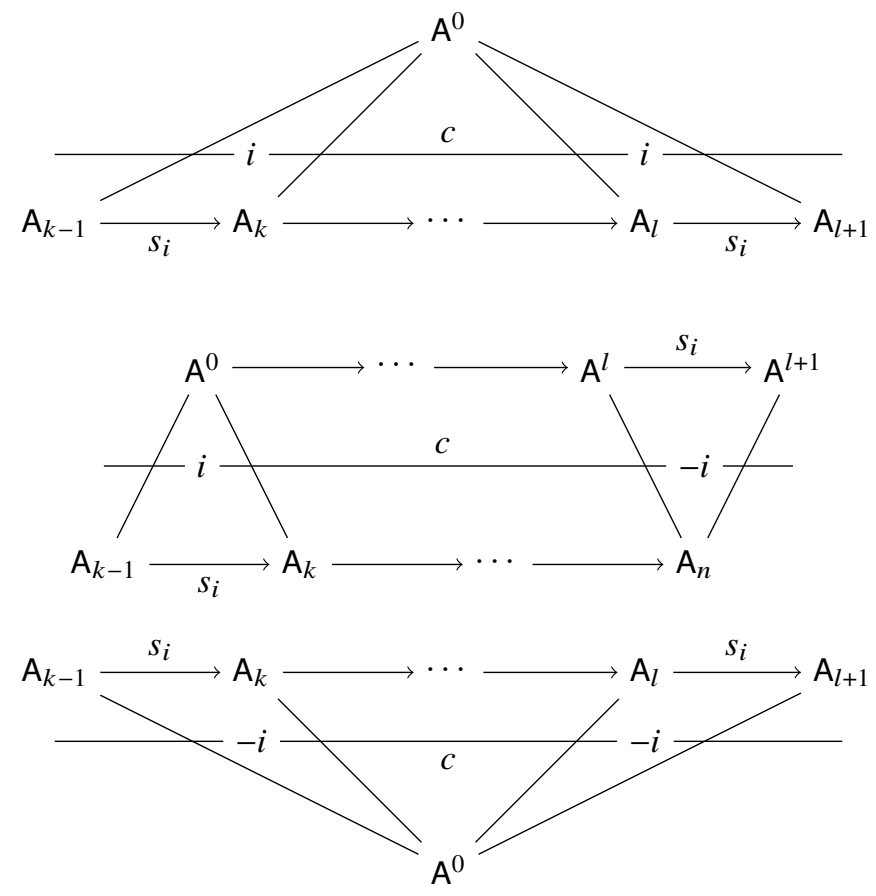
The following lemma is essentially equivalent to Zelevinsky's result on double Bruhat cells [Zel00, Lemma 3.1 (4)]. But because the decorated flag language we use is significantly different from what is in his proof, we rephrase his proofs below for the purpose of completeness.

Lemma 3.37. For a triangulation chosen as above and any closed string $c$ in the corresponding string diagram, the once-mutated cluster $\mathrm{K}_{2}$ variable $A_{c}^{\prime}$ (as an element in $\operatorname{Frac}\left(\mathcal{O}_{d}^{b}\right)$ a priori) belongs to $\mathcal{O}_{d}^{b}$ and $\operatorname{Conf}_{d}^{b}\left(\mathcal{A}_{\mathrm{sc}}\right)$ contains the seed torus $\operatorname{Spec} \mathbb{C}\left[\mathbf{A}_{c}^{ \pm}\right]$.

Proof. Let us consider the top case first.

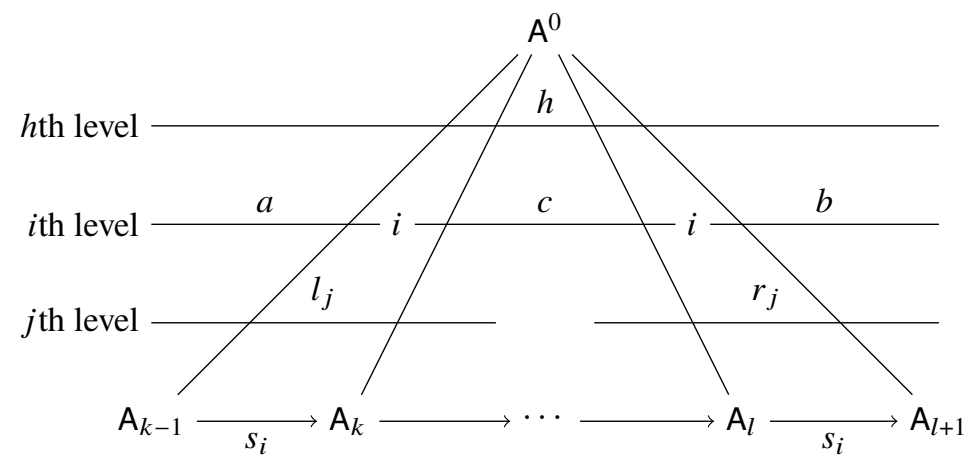

Let $S$ denote the set of indices $j$ whose corresponding simple reflections $s_{j}$ occur between the two $s_{i}$. Let $l_{j}$ and $r_{j}$ denote the strings on level $j$ going across the triangles corresponding to these two $s_{i}$. Then the cluster $\mathrm{K}_{2}$ mutation formula says that

$$
A_{c}^{\prime}=\frac{1}{A_{c}}\left(A_{a} \prod_{j \in S} A_{r_{j}}^{-\mathrm{C}_{j i}}+A_{b} \prod_{j \in S} A_{l_{j}}^{-\mathrm{C}_{j i}}\right)
$$

as a rational function on $\operatorname{Conf}_{d}^{b}\left(\mathcal{A}_{\mathrm{sc}}\right)$. Note that $A_{c}^{\prime}$ is obviously regular outside of the divisor $D_{c}$.

Along the divisor $D_{c}$ we need to do a small trick. Recall from Corollary 3.34 that

$$
\operatorname{ord}_{D_{c}} \Delta_{\omega_{h}}\left(\mathrm{~A}_{k}, \mathrm{~A}^{0}\right)=0
$$

for all $h \neq i$. Let us multiply both sides of the cluster $\mathrm{K}_{2}$ mutation formula by the product $\prod_{h \notin S \cup\{i\}} A_{h}^{-\mathrm{C}_{h i}}$. Now fix a decorated flag $\mathrm{A}^{-1}$ such that $\mathrm{A}^{-1} \stackrel{s_{i}}{\longrightarrow} \mathrm{A}^{0}$ is a compatible pair and $\mathrm{A}_{k-1} \longrightarrow \mathrm{A}^{-1}$ are in general position (not necessarily compatible). Such a decorated flag exists because the decorated double

Bott-Samelson cell associated to the triangle

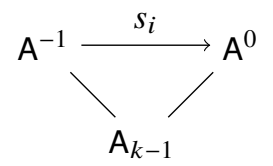

is not empty. Then Corollary 3.26 implies that

$$
\begin{array}{r}
A_{c}^{\prime} \prod_{h \notin S \cup\{i\}} A_{h}^{-C_{h i}=} \frac{1}{A_{c}}\left(A_{a}\left(\Delta_{i}(l+1,-1) \Delta_{i}(l, 0)-\Delta_{i}(l,-1) \Delta_{i}(l+1,0)\right)\right. \\
\left.+A_{b}\left(\Delta_{i}(k,-1) \Delta_{i}(k-1,0)-\Delta_{i}(k-1,-1) \Delta_{i}(k, 0)\right)\right)
\end{array}
$$




$$
\begin{aligned}
& =\frac{1}{A_{c}}\left(A_{a} \Delta_{i}(l+1,-1) A_{c}-A_{a} \Delta_{i}(l,-1) A_{b}\right. \\
& \left.\quad+A_{b} \Delta_{i}(k,-1) A_{a}-A_{b} \Delta_{i}(k-1,-1) A_{c}\right) \\
& =A_{a} \Delta_{i}(l+1,-1)-A_{b} \Delta_{i}(k-1,-1) .
\end{aligned}
$$

The last equality was due to the fact that $\Delta_{i}(l,-1)=\Delta_{i}(k,-1)$ because there is no more $s_{i}$ between the two $s_{i}$. Therefore, we conclude that ${ }^{6}$

$$
A_{c}^{\prime}=\frac{A_{a} \Delta_{i}(l+1,-1)-A_{b} \Delta_{i}(k-1,-1)}{\prod_{h \notin S \cup\{i\}} A_{h}^{-\mathrm{C}_{h i}}} .
$$

Note that the order of vanishing of the denominator $\prod_{h \notin S \cup\{i\}} A_{h}^{-C_{h i}}$ is zero. Therefore, $A_{c}^{\prime}$ is a regular function by the standard codimension 2 argument.

To show that $\operatorname{Conf}_{d}^{b}\left(\mathcal{A}_{\mathrm{sc}}\right)$ contains Spec $\mathbb{C}\left[\mathbf{A}_{c}^{ \pm}\right]$, it suffices to show that we can construct a configuration in $\operatorname{Conf}_{d}^{b}\left(\mathcal{A}_{\mathrm{sc}}\right)$ for any assignment of nonzero numbers to $\left\{A_{c}^{\prime}\right\} \cup\left\{A_{a}\right\}_{a \neq c}$. When the assignment of numbers satisfies

$$
A_{a} \prod_{j \in S} A_{r_{j}}^{-\mathrm{C}_{j i}}+A_{b} \prod_{j \in S} A_{l_{j}}^{-\mathrm{C}_{j i}} \neq 0,
$$

we can reproduce $A_{c}$ from these numbers and we get a unique point in the complement of the divisor $D_{c}$.

When the above nonvanishing condition is not satisfied, $A_{c}=0$ and we need to do a small trick similar to the one we did in proving that $A_{c}^{\prime}$ is regular. First we observe that by using the cluster $\mathrm{K}_{2}$ coordinates on the left of $A_{c}$ (including the ones associated to the left open strings and closed strings that are $<c$ ) we can build a unique configuration

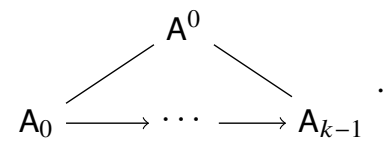

Next we fix a decorated flag $A^{-1}$ the same way as before. Let us now consider the cluster $\mathrm{K}_{2}$ coordinate chart associated to the following triangulation:

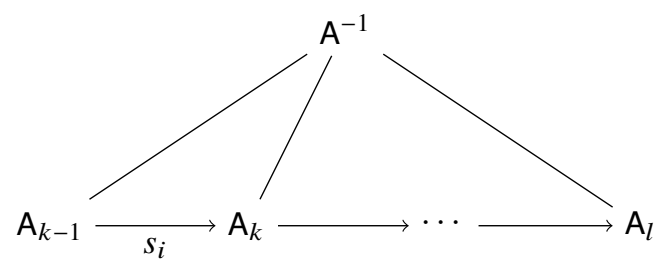

We claim that the nonzero values of $\left\{A_{c}^{\prime}\right\} \cup\left\{A_{a}\right\}_{a \neq c}$ can produce nonzero cluster $\mathrm{K}_{2}$ coordinates associated to the above triangulation.

First let us apply Corollary 3.26 to $\mathrm{A}^{-1} \stackrel{s_{i}}{\longrightarrow} \mathrm{A}^{0}$ and $\mathrm{A}_{k-1} \stackrel{s_{i}}{\longrightarrow} \mathrm{A}_{k}$; the assumption $A_{c}=$ $\Delta_{i}(k, 0)=0$ reduces the identity in Corollary 3.26 to

$$
\Delta_{i}(k,-1) A_{a}=\prod_{j \neq i} A_{l_{j}}^{-\mathrm{C}_{j i}} .
$$

Because both $A_{a}$ and $A_{l_{j}}$ are assumed to be nonzero, we can solve for $\Delta_{i}(k,-1)$ using the above identity and the result is still nonzero. This shows $\mathrm{A}_{k}-\mathrm{A}^{-1}$ and produces nonzero values for the cluster $\mathrm{K}_{2}$ coordinates along this diagonal.

${ }^{6}$ It is worth mentioning that $A_{c}^{\prime}$ is independent of the choice of the decorated flag $\mathrm{A}^{-1}$. 
Let $p$ be an integer with $k \leq p \leq l$. By applying Proposition 3.21 to the triangle

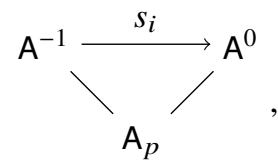
we know that for any $j \neq i, \Delta_{j}(p,-1)=\Delta_{j}(p, 0) \neq 0$. This combined with the fact that $\Delta_{i}(p,-1)=$ $\Delta_{i}(k,-1) \neq 0$ proves that $\mathrm{A}_{p} \longrightarrow \mathrm{A}^{-1}$ and gives nonzero values for all cluster $\mathrm{K}_{2}$ coordinates along these diagonals. Using these nonzero cluster $K_{2}$ coordinates in the triangulation (3.39), we can uniquely construct decorated flags $A_{k}, A_{k+1}, \ldots, A_{l}$.

Next we rewrite Equation (3.38) as

$$
\Delta_{i}(l+1,-1)=\frac{A_{c}^{\prime} \prod_{h \notin S \cup\{i\}} A_{h}^{-\mathrm{C}_{h i}}+A_{b} \Delta_{i}(k-1,-1)}{A_{a}} .
$$

Note that everything on the right is given already (including $\Delta_{i}(k-1,-1)$ from the choice of $\left.\mathrm{A}^{-1}\right)$. Therefore, we can compute $\Delta_{i}(l+1,-1)$ using this equation. Though this equation does not guarantee that $\Delta_{i}(l+1,-1)$ is nonzero, it is nevertheless a number in $\mathbb{A}^{1}$ because the denominator on the right-hand side is nonzero. Recall from Lemma 3.30 that $\Delta_{i}(l+1,-1)$ parametrises all of the Borel subgroups that are of Tits distance $s_{i}$ away from $\mathrm{A}_{l}$; therefore, it can be used to uniquely determine $\mathrm{A}_{l+1}$. Furthermore, this decorated flag $\mathrm{A}_{l+1}$ must satisfy $\Delta_{j}(l+1,0)=\Delta_{j}(l+1,-1)=A_{r_{j}} \neq 0$ for all $j \neq i$ and $\Delta_{i}(j+1,0)=A_{b} \neq 0$. Therefore, we know that $\mathrm{A}_{l+1} \longrightarrow \mathrm{A}^{0}$.

Once we have the pair of decorated flags $A_{l+1} \longrightarrow A^{0}$, we can then use the cluster $K_{2}$ coordinates

on the right of $A_{c}$ to build the remaining decorated flags reconstruction of the configuration from the nonzero numerical assignments to the cluster $\mathrm{K}_{2}$ variables $\left\{A_{c}^{\prime}\right\} \cup\left\{A_{a}\right\}_{a \neq c}$.

Next let us consider the middle case.

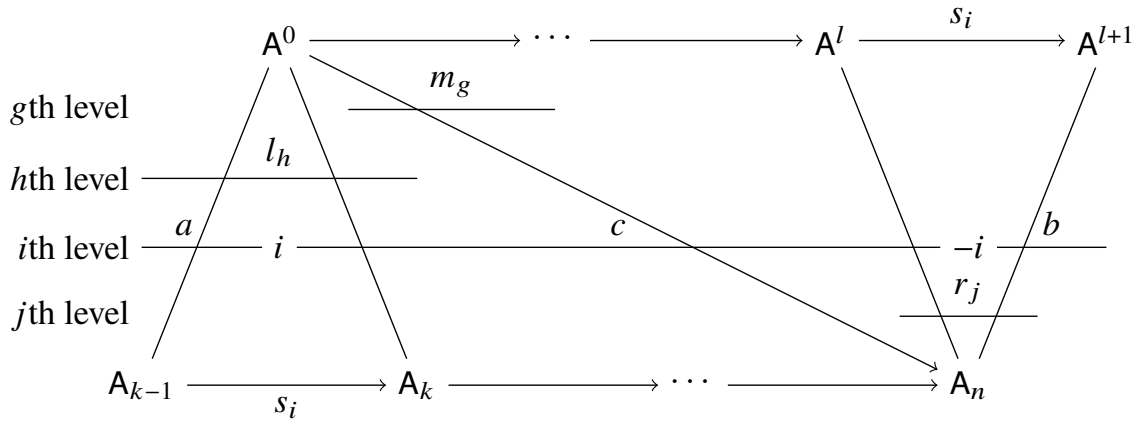

Let $S_{-}$denote the set of indices $h$ whose corresponding simple reflections $s_{h}$ occur after the last $s_{i}$ along the bottom edge of the trapezoid and let $S_{+}$denote the set of indices $j$ whose corresponding simple reflections $s_{j}$ occur before the first $s_{i}$ along the top edge of the trapezoid. Let $l_{h}$ denote the string on level $h \in S_{-}$, going across the triangle corresponding to the left $s_{i}$; let $r_{j}$ denote the string on level $j \in S_{+}$, going across the triangle corresponding to the right $s_{i}$; let $m_{g}$ denote the string on level $g \in S_{+} \cap S_{-}$, going across the diagonal separating the upward pointing and downward pointing triangles. Then the 
cluster $\mathrm{K}_{2}$ mutation formula says that

$$
A_{c}^{\prime}=\frac{1}{A_{c}}\left(A_{a} A_{b} \prod_{g \in S_{+} \cap S_{-}} A_{m_{g}}^{-\mathrm{C}_{g i}}+\left(\prod_{h \in S_{-}} A_{l_{h}}^{-\mathrm{C}_{h i}}\right)\left(\prod_{j \in S_{+}} A_{r_{j}}^{-\mathrm{C}_{j i}}\right)\left(\prod_{g \notin S_{-} \cup S_{+} \cup\{i\}} A_{m_{g}}^{-\mathrm{C}_{g i}}\right)\right) .
$$

Note that $A_{c}^{\prime}$ is obviously regular outside of the divisor $D_{c}$.

Along the divisor $D_{c}$ we again need to do a small trick. First note that by Corollary 3.34,

$$
\operatorname{ord}_{D_{c}}\left(\prod_{g \notin\left(S_{+} \cap S_{-}\right) \cup\{i\}} A_{m_{g}}^{-\mathrm{C}_{g i}}\right)=\operatorname{ord}_{D_{c}}\left(\left(\prod_{h \notin S_{-} \cup\{i\}} A_{l_{h}}^{-\mathrm{C}_{h i}}\right)\left(\prod_{j \in S_{-} \backslash S_{+}} A_{r_{j}}^{-\mathrm{C}_{j i}}\right)\right)=0 .
$$

Let us call this product $M$. We multiply both sides of Equation (3.40) by $M$ and then try to do some simplification. Fix a decorated flag $A^{-1}$ such that $A^{-1} \stackrel{s_{i}}{\longrightarrow} A^{0}$ is a compatible pair and $A_{k-1} \longrightarrow A^{-1}$ (not necessarily compatible) and fix a decorated flag $A_{n+1}$ such that $A_{n} \stackrel{s_{i}}{\longrightarrow} A_{n+1}$ is a compatible pair and $A^{l+1} \longrightarrow A_{n+1}$ are in general position (not necessarily compatible). Then by Corollary 3.26 we have

$$
\begin{aligned}
& M \prod_{g \in S_{+} \cap S_{-}} A_{m_{g}}^{-\mathrm{C}_{g i}}=\Delta_{i}(n+1,-1) \Delta_{i}(n, 0)-\Delta_{i}(n,-1) \Delta_{i}(n+1,0), \\
& M\left(\prod_{h \in S_{-}} A_{l_{h}}^{-\mathrm{C}_{h i}}\right)\left(\prod_{j \in S_{+}} A_{r_{j}}^{-\mathrm{C}_{j i}}\right)\left(\prod_{g \notin S_{-} \cup S_{+} \cup\{i\}} A_{m_{g}}^{-\mathrm{C}_{g i}}\right) \\
= & M\left(\prod_{h \in S_{-}} A_{l_{h}}^{-\mathrm{C}_{h i}}\right)\left(\prod_{j \notin\left(S_{-} \backslash S_{+}\right) \cup\{i\}} A_{r_{j}}^{-\mathrm{C}_{j i}}\right) \\
= & \left(\prod_{h \notin S_{-} \cup\{i\}} A_{l_{h}}^{-\mathrm{C}_{h i}}\right)\left(\prod_{j \in S_{-} \backslash S_{+}} A_{r_{j}}^{-\mathrm{C}_{j i}}\right)\left(\prod_{h \in S_{-}} A_{l_{h}}^{-\mathrm{C}_{h i}}\right)\left(\prod_{j \notin\left(S_{-} \backslash S_{+}\right) \cup\{i\}} A_{r_{j}}^{-C_{j i}}\right) \\
= & \left(\prod_{h \neq i} \Delta_{h}(k, 0)^{-\mathrm{C}_{h i}}\right)\left(\prod_{j \neq i} \Delta_{j}(n, l)^{-\mathrm{C}_{j i}}\right) \\
= & \left(\Delta_{i}(k,-1) \Delta_{i}(k-1,0)-\Delta_{i}(k-1,-1) \Delta_{i}(k, 0)\right) \\
& \left(\Delta_{i}(n+1, l) \Delta_{i}(n, l+1)-\Delta_{i}(n, l) \Delta_{i}(n+1, l+1)\right) .
\end{aligned}
$$

Plugging these into $M$ times Equation (3.40) and remembering

$$
\begin{aligned}
\Delta_{i}(k,-1) & =\Delta_{i}(n,-1), \\
\Delta_{i}(n+1,0) & =\Delta_{i}(n+1, l), \\
\Delta_{i}(k, 0)=\Delta_{i}(n, 0) & =\Delta_{i}(n, l)=A_{c},
\end{aligned}
$$

we get that

$$
\begin{aligned}
M A_{c}^{\prime}= & A_{a} A_{b} \Delta_{i}(n+1,-1)-A_{b} \Delta_{i}(k-1,-1) \Delta_{i}(n+1, l) \\
& -A_{a} \Delta_{i}(k,-1) \Delta_{i}(n+1, l+1)+A_{c} \Delta_{i}(k-1,-1) \Delta_{i}(n+1, l+1) \\
= & \operatorname{det}\left(\begin{array}{ccc}
A_{a} & \Delta_{i}(n+1, l) & A_{c} \\
\Delta_{i}(k-1,-1) & \Delta_{i}(n+1,-1) & \Delta_{i}(k,-1) \\
0 & \Delta_{i}(n+1, l+1) & A_{b}
\end{array}\right) .
\end{aligned}
$$


Note that along the divisor $D_{c}$, the upper right-hand corner of the last matrix vanishes. Therefore, we can conclude that along the divisor $D_{c}$,

$$
A_{c}^{\prime}=\frac{\operatorname{det}\left(\begin{array}{ccc}
A_{a} & \Delta_{i}(n+1, l) & 0 \\
\Delta_{i}(k-1,-1) & \Delta_{i}(n+1,-1) & \Delta_{i}(k,-1) \\
0 & \Delta_{i}(n+1, l+1) & A_{b}
\end{array}\right)}{\prod_{g \notin\left(S_{+} \cap S_{-}\right) \cup\{i\}} A_{m_{g}}^{-C_{g i}}}
$$

is a regular function as well.

To show that $\operatorname{Conf}_{d}^{b}\left(\mathcal{A}_{\mathrm{sc}}\right)$ contains Spec $\mathbb{C}\left[\mathbf{A}_{c}^{ \pm}\right]$, it suffices to show that we can construct a configuration in $\operatorname{Conf}_{d}^{b}\left(\mathcal{A}_{\mathrm{sc}}\right)$ for any assignment of nonzero numbers to $\left\{A_{c}^{\prime}\right\} \cup\left\{A_{a}\right\}_{a \neq c}$. Most of the arguments are similar to the top case, so we will be brief. There is nothing to show when

$$
A_{a} A_{b} \prod_{g \in S_{+} \cap S_{-}} A_{m_{g}}^{-\mathrm{C}_{g i}}+\left(\prod_{h \in S_{-}} A_{l_{h}}^{-\mathrm{C}_{h i}}\right)\left(\prod_{j \in S_{+}} A_{r_{j}}^{-\mathrm{C}_{j i}}\right)\left(\prod_{g \notin S_{-} \cup S_{+} \cup\{i\}} A_{m_{g}}^{-\mathrm{C}_{g i}}\right) \neq 0
$$

because we can already recover a nonzero value for $A_{c}$. When this nonvanishing condition fails, $A_{c}=0$ and we have to do a small trick again. In fact, we only need to focus on the parallelogram

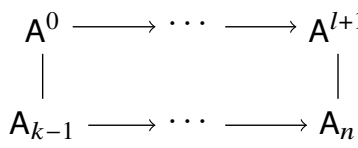

because everything outside can be constructed from the given values of

cluster $K_{2}$ variables as usual. Therefore, it suffices to show that starting from a given $A^{0}-A_{k-1}$ we can construct the rest of the decorated flags in the parallelogram based on the nonzero values of the given cluster $\mathrm{K}_{2}$ variables.

With the decorated flag $A^{-1}$, we can find unique decorated flags $A_{k}, A_{k+1}, \ldots, A_{n}$ using the $K_{2}$ cluster associated to the following triangle:

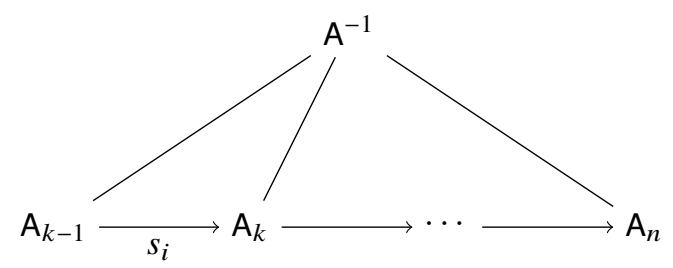

With the decorated flag $A_{n+1}$, we can find unique decorated flags $A^{1}, A^{2}, \ldots, A^{l}$ using the $K_{2}$ cluster associated to the following triangle:

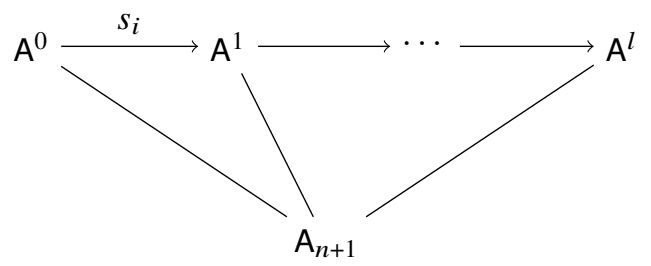

To determine the last decorated flag $\mathrm{A}^{l+1}$, we need to compute $\Delta_{i}(n+1, l+1)$ using Equation (3.41); note that the numerical value of everything else in that equation is already given and the coefficient of $\Delta_{i}(n+1, l+1)$ is $A_{a} \Delta_{i}(k,-1)$, which is nonzero. Therefore, we get $\Delta_{i}(n+1, l+1)$ as an $\mathbb{A}^{1}$ number, which, combined with $\mathrm{A}_{n+1} \longrightarrow \mathrm{A}^{l}$, determines the decorated flag $\mathrm{A}^{l+1}$ uniquely.

We will omit the proof for the bottom case because it is completely analogous to the top case. 
Proposition 3.42. The union $U:=\operatorname{Spec} \mathbb{C}\left[\mathbf{A}_{0}^{ \pm}\right] \cup \bigcup_{c} \operatorname{Spec} \mathbb{C}\left[\mathbf{A}_{c}^{ \pm}\right]$is of codimension at least 2 in $\operatorname{Conf}_{d}^{b}\left(\mathcal{A}_{\mathrm{sc}}\right)$.

Proof. Let $U_{0}:=\operatorname{Spec} \mathbb{C}\left[\mathbf{A}_{0}^{ \pm}\right]$and let $U_{c}:=\operatorname{Spec} \mathbb{C}\left[\mathbf{A}_{c}^{ \pm}\right]$. Note that

$$
\begin{aligned}
\operatorname{Conf}_{d}^{b}\left(\mathcal{A}_{\mathrm{sc}}\right) \backslash U & \left.=\left(\bigcup_{c \text { unfrozen }} D_{c}\right) \cap\left(\bigcup_{e \text { unfrozen }} U_{e}\right)^{c}\right) \\
& =\left(\bigcup_{c \text { unfrozen }} D_{c}\right) \cap\left(\bigcap_{e \text { unfrozen }}\left(U_{e}\right)^{c}\right) \\
& =\bigcup_{c \text { unfrozen }}\left(D_{c} \cap\left(\bigcap_{e \text { unfrozen }}\left(U_{e}\right)^{c}\right)\right) \\
& \subset \bigcup_{c \text { unfrozen }}\left(D_{c} \cap\left(U_{c}\right)^{c}\right) .
\end{aligned}
$$

From the proof of the Lemma 3.37 we see that $D_{c} \cap U_{c}$ is a nonempty open subset of $D_{c}$. Because $D_{c}$ is irreducible, $D_{c} \cap\left(U_{c}\right)^{c}$ must be at least codimension 1 inside $D_{c}$ and hence at least codimension 2 inside $\operatorname{Conf}_{d}^{b}\left(\mathcal{A}_{\mathrm{sc}}\right)$.

To prove $\mathcal{O}_{d}^{b} \cong$ up $\left(\mathscr{A}_{d}^{b}\right)$, we still need to verify the surjectivity condition on the canonical map $p$. We will do so by realising the map $p: T_{\mathscr{A} ; \mathbf{s}_{0}} \rightarrow T_{\mathscr{X}^{\mathrm{uf}} ; \mathrm{s}_{0}^{\mathrm{uf}}}$ as a restriction of the composition $\operatorname{Conf}_{d}^{b}\left(\mathcal{A}_{\mathrm{sc}}\right) \rightarrow$ $\operatorname{Conf}_{d}^{b}\left(\mathcal{A}_{\mathrm{ad}}\right) \rightarrow \operatorname{Conf}_{d}^{b}(\mathcal{B})$.

Proposition 3.43. Consider the surjective map $\pi: \operatorname{Conf}_{d}^{b}\left(\mathcal{A}_{\mathrm{sc}}\right) \rightarrow \operatorname{Conf}_{d}^{b}\left(\mathcal{A}_{\mathrm{ad}}\right)$. For any closed string $c$,

$$
\pi^{*}\left(X_{c}\right)=\prod_{a} A_{a}^{\epsilon_{c a}}
$$

where $\epsilon_{c a}$ is the exchange matrix for the given seed.

Proof. Note that it suffices to prove this statement on one seed. Let us again use the seed as in Lemma 3.37.

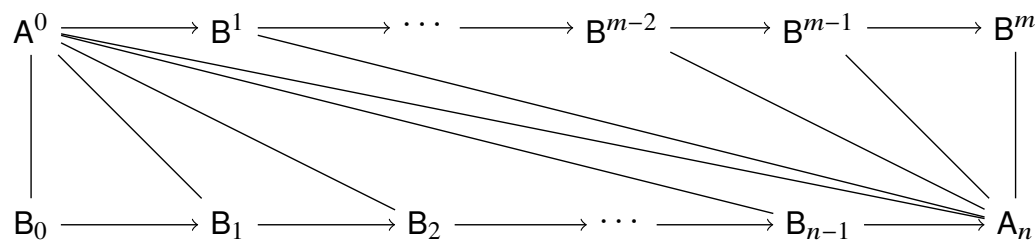

Then the closed strings again come in three types, as described in (3.36). Let us first look at the top case.

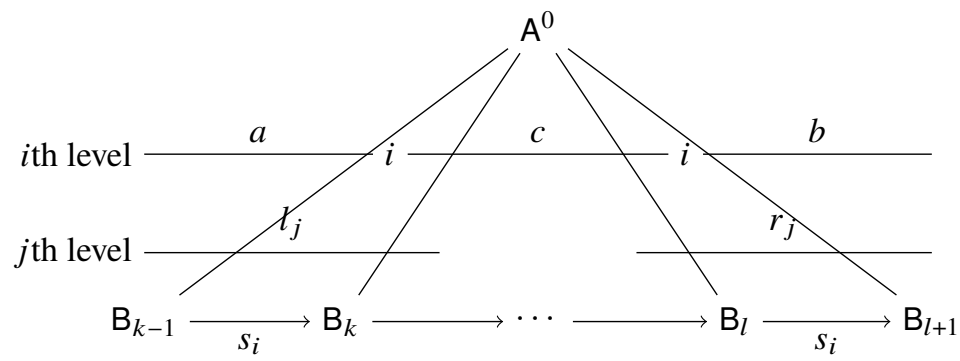


To work out the cluster Poisson coordinate $X_{c}$, we need to first compute the two Lusztig factorisation coordinates at the two ends of the closed string $c$. Consider the triangle containing the left endpoint. By acting by some $u \in \mathrm{U}_{+}$we can move the chosen representative configuration into a configuration with $\mathrm{B}_{k-1}=\mathrm{B}_{-}$and $\mathrm{A}^{0}=\mathrm{U}_{+}$. Because the cluster $\mathrm{K}_{2}$ coordinates are invariant under the $\mathrm{G}$-action, by Corollary 3.22 we see that the unipotent element that moves $\mathrm{B}_{k-1}$ to $\mathrm{B}_{k}$ is $e_{i}\left(\frac{\prod_{j \neq i} A_{l_{j}}^{-\mathrm{C}_{j i}}}{A_{a} A_{c}}\right)$ and hence the Lusztig factorisation coordinate on the left is $q=\frac{\prod_{j \neq i} A_{l_{j}}^{-C_{j i}}}{A_{a} A_{c}}$. By a similar argument, we get that the Lusztig factorisation coordinate on the right is $q^{\prime}=\frac{\prod_{j \neq i} A_{r_{j}}^{-C_{j i}}}{A_{b} A_{c}}$. Therefore, we obtain

$$
\pi^{*}\left(X_{c}\right)=\frac{q^{\prime}}{q}=\frac{A_{a} \prod_{j \neq i} A_{r_{j}}^{-C_{j i}}}{A_{b} \prod_{j \neq i} A_{l_{j}}^{-\mathrm{C}_{j i}}}=\prod_{a} A_{a}^{\epsilon_{c a}}
$$

For the middle case, the Lusztig factorisation coordinate on the left can be computed the same as above, which is $q=\frac{\prod_{j \neq i} A_{l_{j}}^{-\mathrm{C}_{j i}}}{A_{a} A_{c}}$.

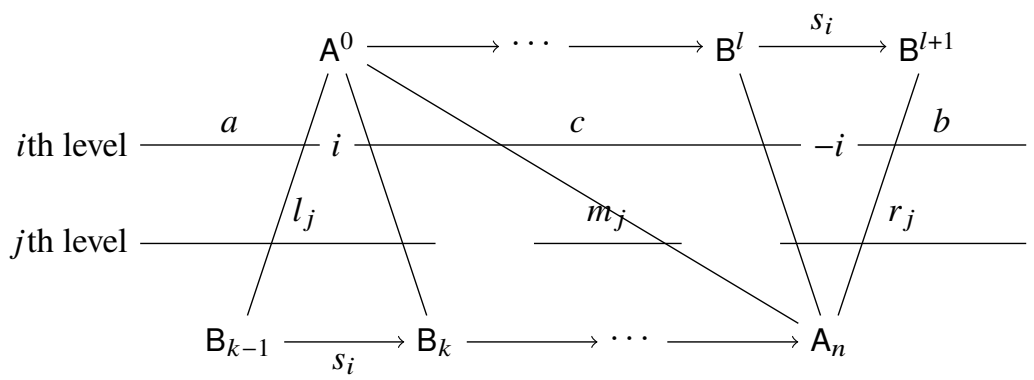

However, the Lusztig factorisation coordinate on the right is slightly more complicated to compute. Recall from Corollary 3.22 that when triangles switch orientation, one also needs to move the whole configuration by the maximal torus element $t:=\prod_{j=1}^{\tilde{r}} \mathrm{~A}_{m_{j}}^{\alpha_{j}^{\vee}}$. But then in the computation of cluster Poisson coordinates on $\operatorname{Conf}_{d}^{b}\left(\mathcal{A}_{\mathrm{ad}}\right)$, we do not allow maximal torus elements appearing in the middle; therefore, we need to push this extra factor of $t$ all the way to the right, resulting in an extra $t^{\alpha_{i}}$ factor (the same technique was also used in the proof of Proposition 3.15). Therefore, the Lusztig factorisation coordinate $p$ is

$$
p=\frac{\prod_{j \neq i} A_{r_{j}}^{-\mathrm{C}_{j i}}}{A_{b} A_{c}} t^{\alpha_{i}}=\frac{\prod_{j \neq i} A_{r_{j}}^{-\mathrm{C}_{j i}}}{A_{b} A_{c}} \frac{A_{c}^{2}}{\prod_{j \neq i} A_{m_{j}}^{-\mathrm{C}_{j i}}}=\frac{A_{c} \prod_{j \neq i} A_{r_{j}}^{-\mathrm{C}_{j i}}}{A_{b} \prod_{j \neq i} A_{m_{j}}^{-\mathrm{C}_{j i}}} .
$$

Now by the definition of cluster Poisson coordinates, we find that

$$
\pi^{*}\left(X_{c}\right)=\frac{1}{p q}=\frac{A_{a} A_{b} \prod_{j \neq i} A_{m_{j}}^{-\mathrm{C}_{j i}}}{\left(\prod_{j \neq i} A_{l_{j}}^{-\mathrm{C}_{j i}}\right)\left(\prod_{j \neq i} A_{r_{j}}^{-\mathrm{C}_{j i}}\right)}=\prod_{a} A_{a}^{\epsilon_{c a}}
$$

The bottom case can be either computed analogous to the top case or obtained from the top case using the transposition morphism as an intertwiner. 
Proposition 3.44. Consider the seed as in Lemma 3.37. Let $m_{j}$ be the string that passes through the diagonal $\mathrm{A}_{n}-\mathrm{A}^{0}$. For an open string $f$ on the ith level next to a node $n$, denote the open strings on the same side as $f$ and on the jth level with $j \neq i$ by $a_{j}$ and denote the string on the other side of $f$ by $b$. Then we have

$$
\pi^{*}\left(X_{f}\right)=\left\{\begin{array}{l}
\frac{\prod_{j \neq i} A_{a_{j}}^{-C_{j i}}}{A_{b} A_{f}} \\
\frac{A_{b} \prod_{j \neq i} A_{m_{j}}^{-\mathrm{C}_{j i}}}{A_{f} \prod_{j \neq i} A_{a_{j}}^{-C_{j i}}} \text { if } \frac{f}{f}-i \frac{b}{b} \text { or } \frac{b}{b} i \frac{f}{-} .
\end{array}\right.
$$

If $f$ is a string on the ith level that is open on both ends (which typically happens for $i>r$ ), then we have

$$
\pi^{*}\left(X_{f}\right)=\prod_{j=1}^{\tilde{r}} A_{m_{j}}^{\mathrm{C}_{j i}}
$$

In particular, these formulas are all in the form

$$
\pi^{*}\left(X_{f}\right)=\left(\prod_{c \text { closed }} A_{c}^{\epsilon_{f c}}\right) \cdot\left(\text { Laurent monomial in } A_{g} \text { for open string } g\right)
$$

Proof. The cases where $f$ is on the left side of the string diagram with a node attached can be obtained in the same way as in the proof of the last proposition. The cases where $f$ is on the right side of the string diagram with a node attached can then be obtained from the cases on the left via the transposition morphism. It remains to show the case where $f$ is open on both ends, for which

$$
\pi^{*}\left(X_{f}\right)=t^{\alpha_{i}}=t^{\sum_{j=1}^{\tilde{r}} \mathrm{C}_{j i} \omega_{j}}=\prod_{j=1}^{\tilde{r}} A_{m_{j}}^{\mathrm{C}_{j i}}
$$

Our chosen triangulation determines a cluster Poisson seed torus $T_{\mathscr{X}}$ on $\operatorname{Conf}_{d}^{b}\left(\mathcal{A}_{\mathrm{ad}}\right)$ and a cluster $\mathrm{K}_{2}$ seed torus $T_{\mathscr{A}}$ on $\operatorname{Conf}_{d}^{b}\left(\mathcal{A}_{\mathrm{sc}}\right)$. Because these two seed tori are both cut out by the same general position conditions on the underlying undecorated flags, the surjective map $\pi: \operatorname{Conf}_{d}^{b}\left(\mathcal{A}_{\mathrm{sc}}\right) \rightarrow \operatorname{Conf}_{d}^{b}\left(\mathcal{A}_{\mathrm{ad}}\right)$ restricts to a surjective map $\pi: T_{\mathscr{A}} \rightarrow T_{\mathscr{X}}$.

Moreover, the formulas from Proposition 3.43 and Proposition 3.44 showed that $\pi$ fits in the following commutative diagram (where the maps $e, f, p$ and $q$ are defined in Appendix A.2):

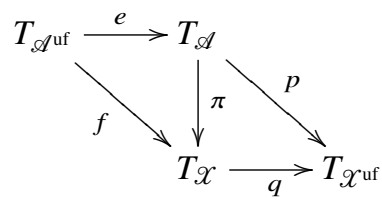

In particular, this diagram is just a restriction of the maps in Diagram (2.25) to some open subsets corresponding to our choice of triangulation.

Because $p=q \circ \pi$ and both $q$ and $\pi$ are surjective, we know that $p$ is surjective as well. By combining the surjectivity of $p$ and the codimension 2 condition (Proposition 3.42), we finally prove our first claim at the beginning of the section. 
Theorem 3.45. $\mathcal{O}_{d}^{b} \cong \operatorname{up}\left(\mathscr{A}_{d}^{b}\right)$.

Let us look at the other claim at the beginning of this subsection, $\mathcal{O}\left(\operatorname{Conf}_{d}^{b}\left(\mathcal{A}_{\mathrm{ad}}\right)\right) \cong \mathrm{up}\left(X_{d}^{b}\right)$. We will use a technique similar to the proof of [SW20, Theorem 2.15].

First note that from the commutation diagram above we get an injective algebra homomorphism $\pi^{*}: \operatorname{up}\left(\mathscr{X}_{d}^{b}\right) \rightarrow \operatorname{up}\left(\mathscr{A}_{d}^{b}\right)$ (see Proposition A.28 and Proposition A.30). In particular, an element $F \in \operatorname{Frac}\left(\operatorname{up}\left(x_{d}^{b}\right)\right)$ is in up $\left(\mathscr{X}_{d}^{b}\right)$ if and only if $\pi^{*}(F)$ is in up $\left(\mathscr{A}_{d}^{b}\right)$.

Theorem 3.46. $\mathcal{O}\left(\operatorname{Conf}_{d}^{b}\left(\mathcal{A}_{\mathrm{ad}}\right)\right) \cong \operatorname{up}\left(\mathscr{X}_{d}^{b}\right)$.

Proof. Because $\operatorname{Conf}_{d}^{b}\left(\mathcal{A}_{\mathrm{ad}}\right)$ contains a cluster Poisson seed torus $T_{\mathscr{X}}$ as an open dense subset, we have $\operatorname{Frac}\left(\mathcal{O}\left(\operatorname{Conf}_{d}^{b}\left(\mathcal{A}_{\mathrm{ad}}\right)\right)\right) \cong \operatorname{Frac}\left(\mathcal{O}\left(T_{\mathscr{X}}\right)\right)=\operatorname{Frac}\left(\operatorname{up}\left(\mathscr{X}_{d}^{b}\right)\right)$, which contains both $\mathcal{O}\left(\operatorname{Conf}_{d}^{b}\left(\mathcal{A}_{\mathrm{ad}}\right)\right)$ and up $\left(\mathscr{X}_{d}^{b}\right)$ as subalgebras. Now consider the following commutative diagram of algebras:

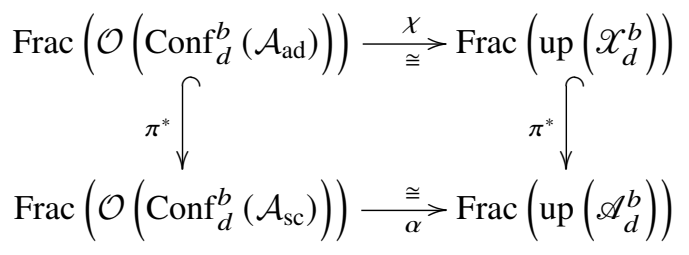

Suppose that $f$ is a regular function on $\operatorname{Conf}_{d}^{b}\left(\mathcal{A}_{\mathrm{ad}}\right)$. Then we know that $\chi(f) \in \operatorname{Frac}\left(\operatorname{up}\left(\mathscr{X}_{d}^{b}\right)\right)$. To show that $\chi(f)$ is actually in up $\left(\mathscr{X}_{d}^{b}\right)$, we only need to show $\pi^{*} \circ \chi(f) \in \operatorname{up}\left(\mathscr{A}_{d}^{b}\right)$ by Proposition A.28. But this is true because $\pi^{*} \circ \chi(f)=\alpha \circ \pi^{*}(f)$ is in the image of the composition

$$
\mathcal{O}\left(\operatorname{Conf}_{d}^{b}\left(\mathcal{A}_{\mathrm{ad}}\right)\right) \stackrel{\pi^{*}}{\longrightarrow} \mathcal{O}\left(\operatorname{Conf}_{d}^{b}\left(\mathcal{A}_{\mathrm{sc}}\right)\right) \stackrel{\alpha}{\longrightarrow} \operatorname{up}\left(\mathscr{A}_{d}^{b}\right) .
$$

On the other hand, suppose that $F$ is an element in up $\left(x_{d}^{b}\right)$. Then we know that $\chi^{-1}(F)$ is a rational function on $\operatorname{Conf}_{d}^{b}\left(\mathcal{A}_{\mathrm{ad}}\right)$. Because $\pi: \operatorname{Conf}_{d}^{b}\left(\mathcal{A}_{\mathrm{sc}}\right) \rightarrow \operatorname{Conf}_{d}^{b}\left(\mathcal{A}_{\mathrm{ad}}\right)$ is surjective, to show the regularity of $\chi^{-1}(F)$, it suffices to show that $\pi^{*} \circ \chi^{-1}(F)$ is a regular function on $\operatorname{Conf}_{d}^{b}\left(\mathcal{A}_{\mathrm{sc}}\right)$. But this is true because $\pi^{*} \circ \chi^{-1}(F)=\alpha^{-1} \circ \pi^{*}(F)$ is in the image of the composition

$$
\operatorname{up}\left(\mathscr{X}_{d}^{b}\right) \stackrel{\pi^{*}}{\longrightarrow} \operatorname{up}\left(\mathscr{A}_{d}^{b}\right) \stackrel{\alpha^{-1}}{\longrightarrow} \mathcal{O}\left(\operatorname{Conf}_{d}^{b}\left(\mathcal{A}_{\mathrm{sc}}\right)\right)
$$

\section{Donaldson-Thomas Transformation of Bott-Samelson Cells}

In this section we will show that the cluster Donaldson-Thomas transformation exists on the unfrozen Poisson cluster algebra up $\left(x_{d}^{b}\right)^{\text {uf }}$ and realise it as a biregular morphism on the undecorated double Bott-Samelson cell $\operatorname{Conf}_{d}^{b}(\mathcal{B})$. We will first show it for the case $\left(\mathscr{X}_{b}^{e}\right)^{\text {uf }}$ by constructing a maximal green sequence, whose existence implies the existence of cluster Donaldson-Thomas sequence (see Appendix A.2 for more details) and then deduce the general cases $\left(x_{d}^{b}\right)^{\text {uf }}$ by using the reflection maps defined in Subsection 2.3.

\subsection{A Maximal Green Sequence for $\left(\mathscr{X}_{b}^{e}\right)^{\mathrm{uf}}$}

Let us consider the cluster Poisson algebra up $\left(\mathscr{X}_{b}^{e}\right)^{\text {uf }}$ associated to the undecorated double BottSamelson cell $\operatorname{Conf}_{b}^{e}(\mathcal{B})$. Our goal is to construct a maximal green sequence. 
We should first fix an initial seed. Due to the fact that the positive braid upstairs is the identity $e$, the trapezoid is in fact a triangle and there is only one available triangulation in this case, which is the following:

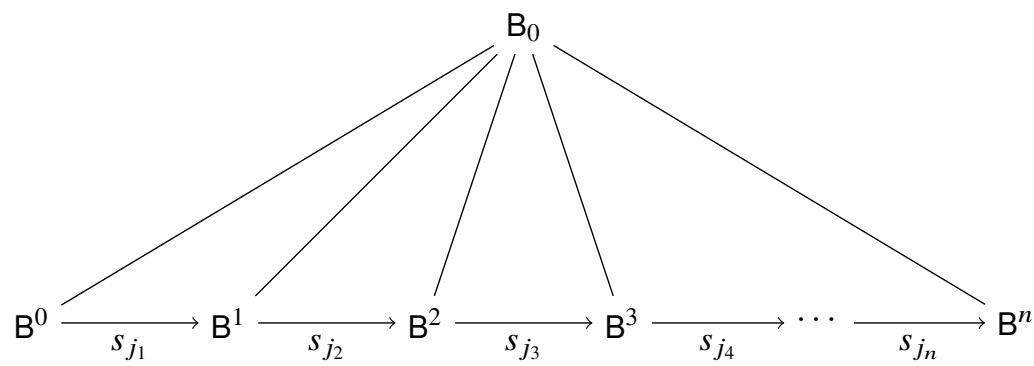

Because all of the triangles are of the shape $\bigwedge_{s_{j}}$, the corresponding string diagram only has simple roots at the nodes. To better demonstrate the idea, let us use the word

$$
(2,1,3,2,1,3,1,3,2,2,1)
$$

as a running example. The string diagram looks like the following (we will only draw the closed strings because open strings are not part of the unfrozen seed).

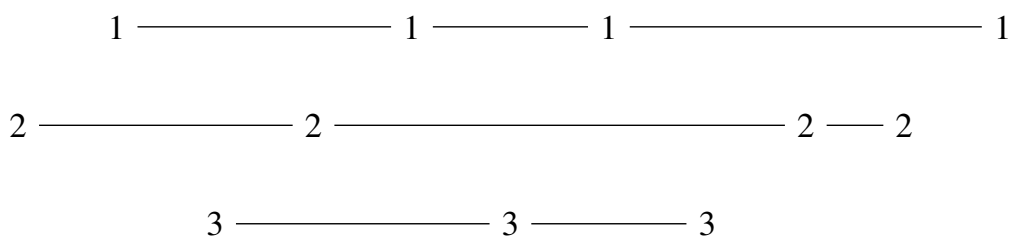

Recall that the closed strings become vertices of the seed $\mathbf{s}^{\mathrm{uf}}$ and the associated exchange matrix $\epsilon$ has two types of nonvanishing entries: \pm 1 for neighbouring vertices on the same horizontal level and $\pm \mathrm{C}_{j i}$ between nearby vertices on the $i$ th and $j$ th levels. Because the magnitude of these entries is determined by the horizontal levels of the vertices, the only extra data we need to record are the signs. We will hence use arrows of the form $a \longrightarrow b$ to denote the first case with $\epsilon_{a b}=-\epsilon_{b a}=1$ and use arrow $a \leadsto b$ to denote the second case with $\epsilon_{a b}>0$ and $\epsilon_{b a}<0$.

Using such notation, our running example gives rise to a seed that can be described as follows:

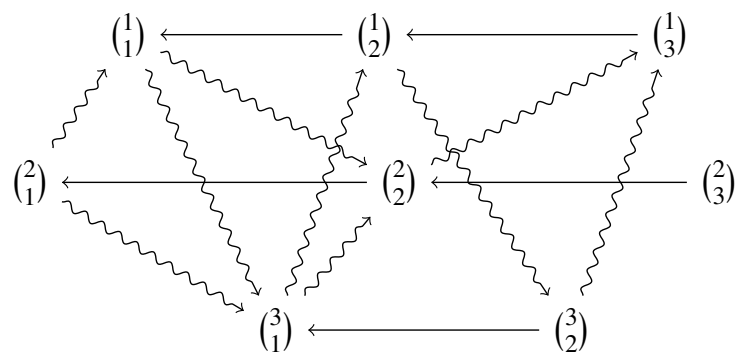

We are now ready to describe a maximal green sequence for seeds of such form. First, for each entry $i_{k}$ of the word $\mathbf{i}=\left(i_{1}, i_{2}, \ldots, i_{n}\right)$, we define

$$
t_{k}:=\#\left\{l \mid k<l \leq n \text { and } i_{l}=i_{k}\right\} .
$$


Note that if $i_{k}$ is the first occurrence of the letter $i$ in the word $\mathbf{i}$, then there are exactly $t_{k}$ many closed strings on level $i$.

Next for each $i_{k}$ we define a mutation sequence

$$
L_{k}:=\mu_{\left(\begin{array}{c}
i_{k} \\
t_{k}
\end{array}\right)} \circ \cdots \circ \mu_{\left(\begin{array}{c}
i_{k} \\
2
\end{array}\right)} \circ \mu_{\left(\begin{array}{c}
i_{k} \\
1
\end{array}\right)} .
$$

In particular, if $i_{k}$ is the last occurrence of the letter $i$ in the word $\mathbf{i}$, then $L_{k}$ is trivial. We then claim the following.

Theorem 4.1. The mutation sequence

$$
L_{n} \circ L_{n-1} \circ \cdots \circ L_{2} \circ L_{1}
$$

is a maximal green sequence.

In the running example, the mutation sequence described in Theorem 4.1 is

$$
\begin{aligned}
& L_{11} \circ L_{10} \circ L_{9} \circ L_{8} \circ L_{7} \circ L_{6} \circ L_{5} \circ L_{4} \circ L_{3} \circ L_{2} \circ L_{1} \\
= & L_{9} \circ L_{7} \circ L_{6} \circ L_{5} \circ L_{4} \circ L_{3} \circ L_{2} \circ L_{1} \\
= & \left(\mu_{\left(\begin{array}{l}
2 \\
1
\end{array}\right)}\right) \circ\left(\mu_{\left(\begin{array}{l}
1 \\
1
\end{array}\right)}\right) \circ\left(\mu_{\left(\begin{array}{l}
3 \\
1
\end{array}\right)}\right) \circ\left(\mu_{\left(\begin{array}{l}
1 \\
2
\end{array}\right)} \circ \mu_{\left(\begin{array}{l}
1 \\
1
\end{array}\right)}\right) \circ\left(\mu_{\left(\begin{array}{l}
2 \\
2
\end{array}\right)} \circ \mu_{\left(\begin{array}{l}
2 \\
1
\end{array}\right)}\right) \circ\left(\mu_{\left(\begin{array}{l}
3 \\
2
\end{array}\right)} \circ \mu_{\left(\begin{array}{l}
3 \\
1
\end{array}\right)}\right) \\
& \circ\left(\mu_{\left(\begin{array}{l}
1 \\
3
\end{array}\right)} \circ \mu_{\left(\begin{array}{l}
1 \\
2
\end{array}\right)} \circ \mu_{\left(\begin{array}{l}
1 \\
1
\end{array}\right)}\right) \circ\left(\mu_{\left(\begin{array}{l}
2 \\
3
\end{array}\right)} \circ \mu_{\left(\begin{array}{l}
2 \\
2
\end{array}\right)} \circ \mu_{\left(\begin{array}{l}
2 \\
1
\end{array}\right)}\right) .
\end{aligned}
$$

Proposition 4.2. In terms of triangles in the triangulation, the mutation sequence $L_{k}$ is equivalent to a reflection map $i_{k}$ r followed by a change of coordinates corresponding to a sequence of mutations that

moves the new triangle

Proof. Recall that if we start with a cluster Poisson coordinate chart on the left, then the reflection morphism maps it to the cluster Poisson coordinate chart on the right.

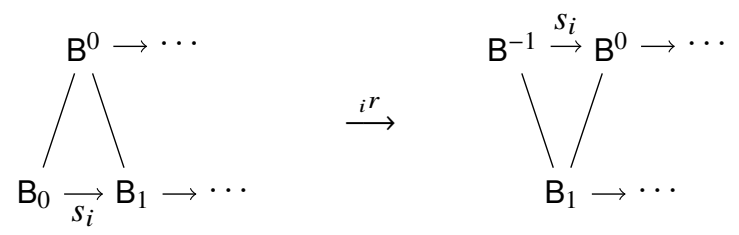

In particular, these two cluster Poisson coordinates have isomorphic unfrozen seeds because the sign change of the leftmost node of the string diagram does not change the exchange matrix entries between closed strings. Now it remains to show that cluster Poisson coordinates pull back to their corresponding cluster Poisson coordinates via ${ }_{i} r^{*}$. But by the definition of cluster Poisson coordinates, it suffices to

show that if the triangle in the picture on the left is

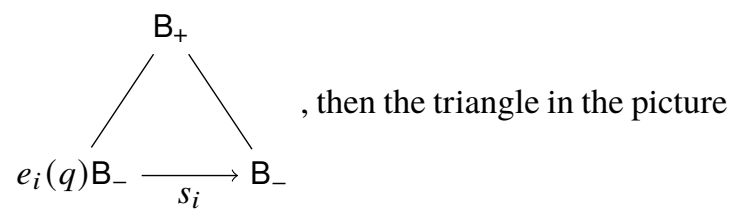

on the right is

$$
e_{-i}\left(q^{-1}\right) \mathrm{B}_{+} \stackrel{s_{i}}{\longrightarrow} \mathrm{B}_{+}
$$

. By the definition of the reflection map ${ }_{i} r$, it suffices to show 
that $e_{i}(q) \mathrm{B}_{-} \frac{s_{i}}{-} e_{-i}\left(q^{-1}\right) B_{+}$. But this follows from the following computation:

$$
\begin{aligned}
\mathrm{B}_{-} e_{i}(q)^{-1} e_{-i}\left(q^{-1}\right) \mathrm{B}_{+} & =\mathrm{B}_{-} e_{i}(-q) e_{-i}\left(q^{-1}\right) \mathrm{B}_{+} \\
& =\mathrm{B}_{-} \bar{s}_{i} q^{-\alpha_{i}^{\vee}} e_{i}(q) \mathrm{B}_{+} \\
& =\mathrm{B}_{-} \bar{s}_{i} \mathrm{~B}_{+} .
\end{aligned}
$$

The last proposition shows that the mutation sequence in Theorem 4.1 defines a biregular map that is a composition of left reflections:

$$
\left(j_{n} r\right) \circ\left(j_{n-1} r\right) \circ \cdots \circ\left({ }_{j_{1}} r\right): \operatorname{Conf}_{b}^{e}(\mathcal{B}) \rightarrow \operatorname{Conf}_{e}^{b^{\circ}}(\mathcal{B})
$$

where $s_{j_{1}} s_{j_{2}} \ldots s_{j_{n}}=b$.

Now it remains to prove Theorem 4.1. For simplicity, let us first investigate what happens when $b=\left(s_{1}, s_{1}, s_{1}, s_{1}\right)$.

It is not hard to see that the initial seed looks like the following:

$$
a \longleftarrow c
$$

From Appendix A.2, we can use auxiliary frozen vertices (principal coefficients) to keep track of the colouring of the vertices. We adopt the convention of labelling the auxiliary frozen vertex connecting to vertex $a$ in the initial seed by $a^{\prime}$.

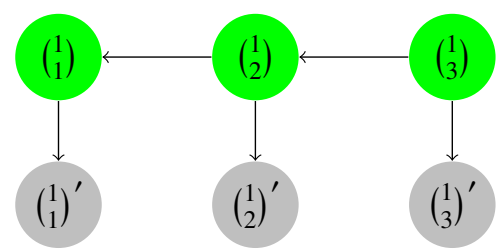

The sequence of mutations for such a seed described in Theorem 4.1 is then $\mu_{a} \circ \mu_{b} \circ \mu_{a} \circ \mu_{c} \circ \mu_{b} \circ \mu_{a}$ and the seed after each mutation looks like the following (going from left to right across each row and going from the top row to the bottom row):
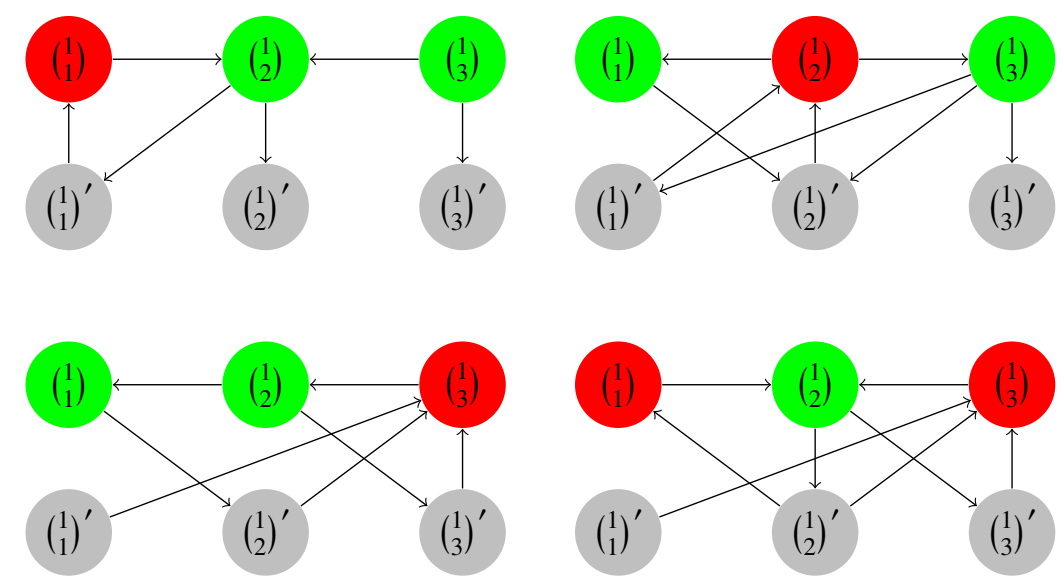

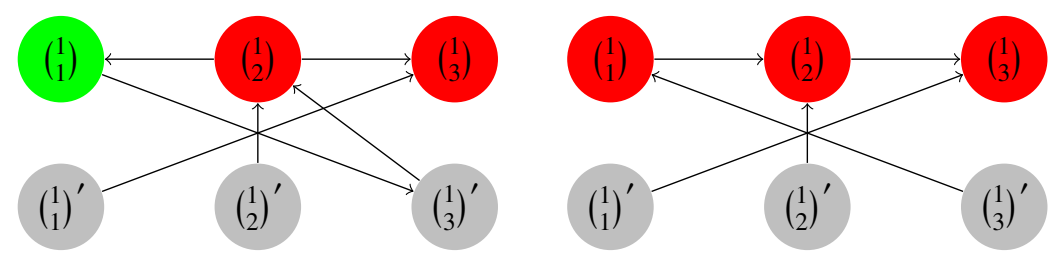

The case of a longer positive braid $b=\left(s_{1}, s_{1}, \ldots, s_{1}\right)$ can be done in a similar way. Before we go into cases with more than one letter, let us first make the following observation for this case.

Proposition 4.3. If $b=\left(s_{1}, s_{1}, \ldots, s_{1}\right)$, then the following is true for the sequence of mutations given by Theorem 4.1:

- $\epsilon_{a b^{\prime}}$ is $-1,0$ or 1 for any two vertices $a$ and $b$.

- In each cycle, the first mutation we do changes the colour of the first green vertex to red (counting from left to right) and then each mutation we do moves this red colour to the right while restoring the green colour for the vertices behind.

- At the end of each cycle, all red vertices are to the right of all green vertices and the separation point is the last node with a simple root labelling (counting from left to right).

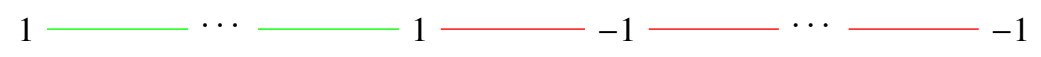

- After going through the mutation sequence in Theorem 4.1 completely, the final seed looks like the following:

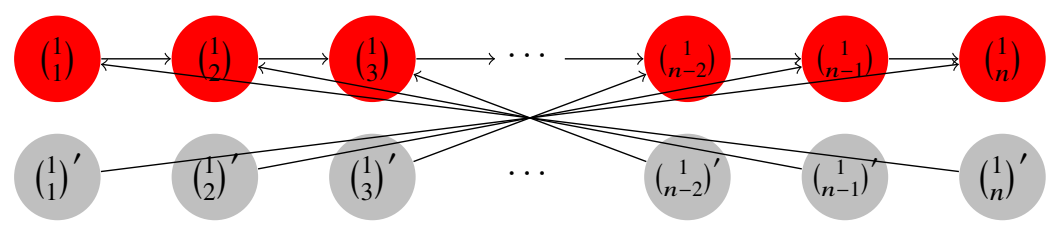

Proof. It follows from an induction on the number of vertices in the seed.

Now let us turn to the more complicated cases with more than one letter. We start with the following proposition.

Proposition 4.4. If a seed comes from a triangulation, then between the ith level and the jth level, the exchange matrix can always be depicted locally by an oriented cycle using the arrow notation as below (plus some degenerate cases):
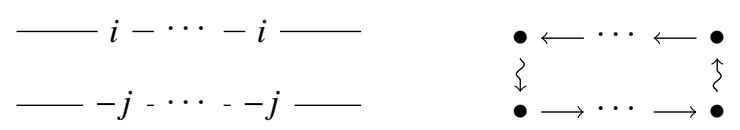

Proof. This statement follows from seed amalgamation.

Remark 4.5. Please be aware that degenerate cases include situations like the following two:
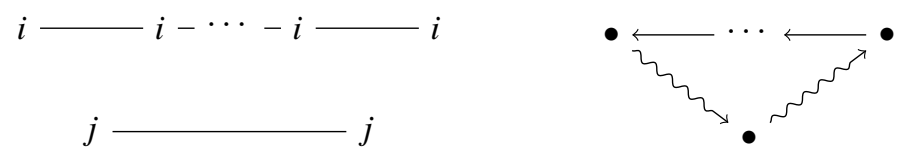

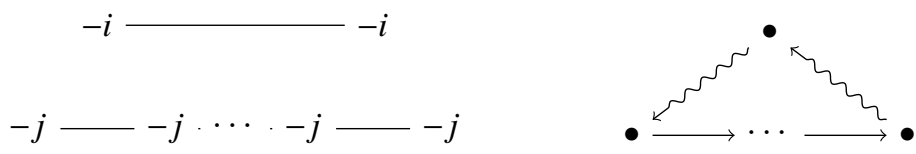

Proposition 4.6. If one mutates according to Theorem 4.1, then the following are true:

- $\epsilon_{a b^{\prime}}=0$ for vertices $a$ and $b$ on different levels.

$\circ \epsilon_{a b^{\prime}}$ is either $-1,0$ or 1 for vertices $a$ and $b$ on the same level.

- Within each cycle, the mutations only change colours of vertices within the level (say the ith) on which the mutations are taking places and they change the colours in the same way as the case of positive braids $\left(s_{i}, s_{i}, \ldots, s_{i}\right)$ (Proposition 4.3).

Proof. We do an induction on the number of mutations. The base case is trivial. For the inductive step, let us first prove the claim ' $\epsilon_{a b^{\prime}}=0$ for vertices $a$ and $b$ on different levels'. At the beginning of each cycle, the vertex we pick for step (1) always looks like the following:
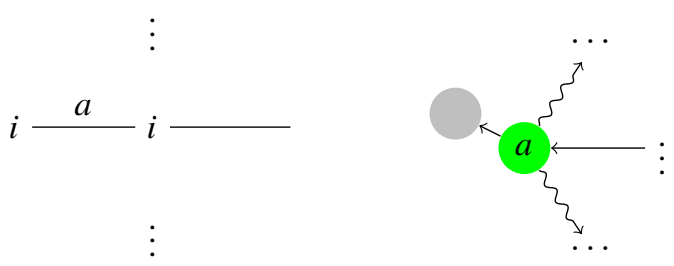

Note that the squiggly arrows going across different levels always go away from vertex $a$. Therefore, when we mutate at $a$, no new arrow will be added between the auxiliary frozen vertices connected to $a$ and other vertices on other levels.

When we are in the middle of a cycle, we know from Proposition 4.4 that the part of the seed we are mutating must look like the following:
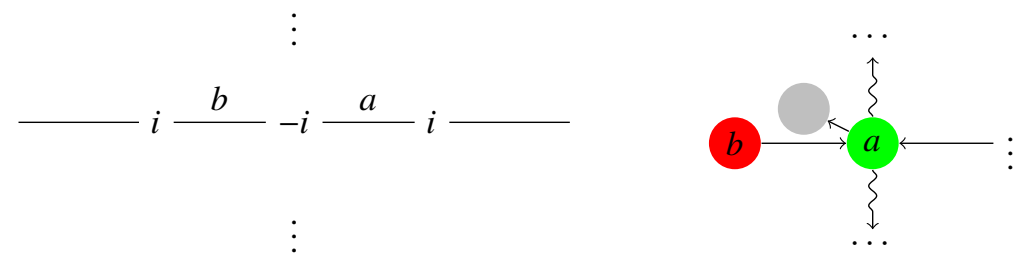

Again, the squiggly arrows going across different levels always point away from vertex $a$. Therefore, when we mutate at $a$, still no new arrow will be added between auxiliary frozen vertices connected to $a$ and other vertices on other levels. Therefore, the claim that $\epsilon_{c d^{\prime}}=0$ for vertices $c$ and $d$ on different levels remains true.

But then because the colour of the vertex $c$ is recorded by $\epsilon_{c d^{\prime}}$, we know that the colour change must only occur at the $i$ th level. The other two claims then follow immediately from Proposition 4.3.

Proof of Theorem 4.1. From our last proposition we know that on each horizontal level the colours of the vertices change in the same way as the single letter case; therefore, it is true that Theorem 4.1 mutates at green vertices only and eventually all green vertices turn red.

\subsection{Cluster Donaldson-Thomas Transformation on $\operatorname{Conf}_{d}^{b}(\mathcal{B})$}

Now we have obtained a maximal green sequence for the seed $\mathbf{i}_{\tau}$ associated to one triangulation of $\operatorname{Conf}_{b}^{e}(\mathcal{B})$, so we are very close to getting a cluster Donaldson-Thomas transformation; all we need 
in addition is an appropriate seed isomorphism that permutes the rows of the $c$-matrix, making it into - id.

Recall from Proposition 4.2 that the maximal green sequence given in Theorem 4.1 can be thought of as a sequence of left reflections. So after completing the mutation sequence in Theorem 4.1, we get a triangulation for $\operatorname{Conf}_{e}^{b^{\circ}}(\mathcal{B})$. This implies that we need some cluster isomorphism that maps $\operatorname{Conf}_{e}^{b^{\circ}}(\mathcal{B})$ back to $\operatorname{Conf}_{b}^{e}(\mathcal{B})$. One obvious choice is the transposition map $(-)^{t}$ from Subsection 2.3. We know from Proposition 3.15 that the transposition map is indeed a cluster isomorphism and combinatorially it is indeed a seed isomorphism that maps the seed after the maximal green sequence back to the initial seed with the property that the $c$-matrix is permuted into -id.

Combining the transposition map with the maximal green sequence from the last subsection, we can now describe the cluster Donaldson-Thomas transformation on the double Bott-Samelson cell $\operatorname{Conf}_{b}^{e}(\mathcal{B})$ geometrically, which is the composition

$$
\mathrm{DT}: \operatorname{Conf}_{b}^{e}(\mathcal{B}) \stackrel{\begin{array}{c}
\text { a sequence of } \\
\text { left reflections }
\end{array}}{\longrightarrow} \operatorname{Conf}_{e}^{b^{\circ}}(\mathcal{B}) \longrightarrow \operatorname{Conf}_{b}^{e}(\mathcal{B})
$$

Example 4.7. Let us do another example to state in more details how the cluster Donaldson-Thomas transformation works. Take $b=s_{1} s_{2} s_{3}$ and the cluster Donaldson-Thomas transformation on $\operatorname{Conf}_{b}^{e}(\mathcal{B})$ is given by the following operations:
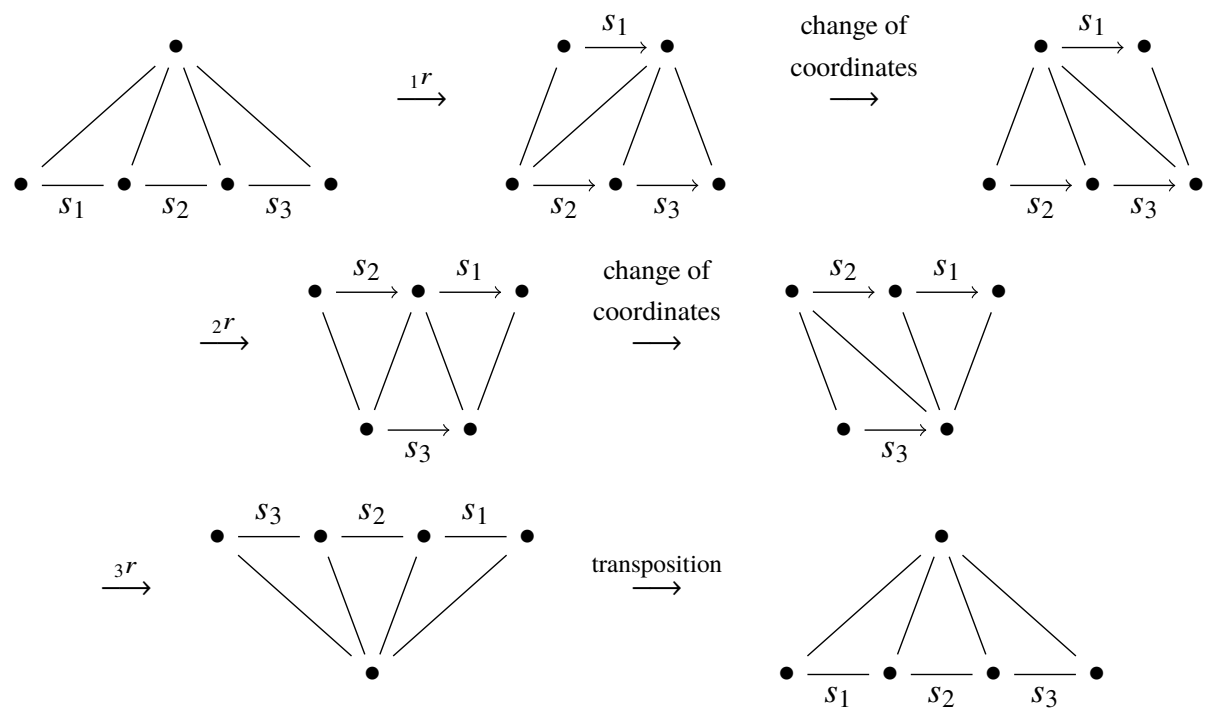

Let us now look at the general case for double Bott-Samelson cell $\operatorname{Conf}_{d}^{b}(\mathcal{B})$. By a similar argument as in Proposition 4.2, one can prove that reflection maps $r^{i}$ are more than just biregular maps: they are cluster isomorphisms that preserve the cluster structures on the undecorated double Bott-Samelson cells. To be more precise, suppose that $b=s_{i_{1}} s_{i_{2}} \ldots s_{i_{m}}$. Consider the biregular map $\eta:=r^{i_{1}} \circ r^{i_{2}} \circ \cdots \circ r^{i_{m}}$. This is not only a biregular map from $\operatorname{Conf}_{d}^{b}(\mathcal{B})$ onto $\operatorname{Conf}_{d b^{\circ}}^{e}(\mathcal{B})$ but also a cluster isomorphism between up $\left(X_{d}^{b}\right)^{\text {uf }}$ and up $\left(X_{d b^{\circ}}^{e}\right)^{\text {uf }}$, whose cluster Donaldson-Thomas transformation was shown in the previous sections.

Because $\eta$ is a cluster isomorphism, by a result of Goncharov and Shen (Theorem A.36), we know that the cluster Donaldson-Thomas transformation on $\operatorname{Conf}_{d}^{b}(\mathcal{B})$ can be obtained from that of $\operatorname{Conf}_{d b^{\circ}}^{e}(\mathcal{B})$ as

$$
\mathrm{DT}_{\operatorname{Conf}_{d}^{b}(\mathcal{B})}=\eta^{-1} \circ \mathrm{DT}_{\mathrm{Conf}_{d b^{\circ}}^{e}(\mathcal{B})} \circ \eta .
$$


Let us draw a picture to describe what such composition really does. We begin with a triangulation associated to the pair $(b, d)$ where the first set of triangles are of the shape

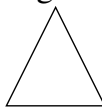
(corresponding to letters of $d$ ) and the second set of triangles are of the shape

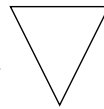
(corresponding to letters of $b$ ).

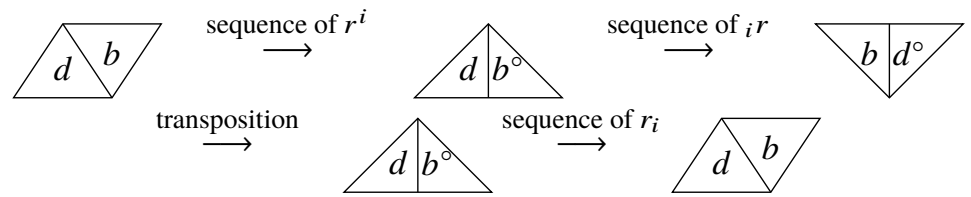

There are some further simplifications that we can perform: first, we can use Proposition 2.29 to turn each $r_{i} \circ(-)^{t}$ into $(-)^{t} \circ{ }^{i} r$; then we can cancel the left reflections ${ }_{i} r$ and ${ }^{i} r$, which yields the following simplified version:

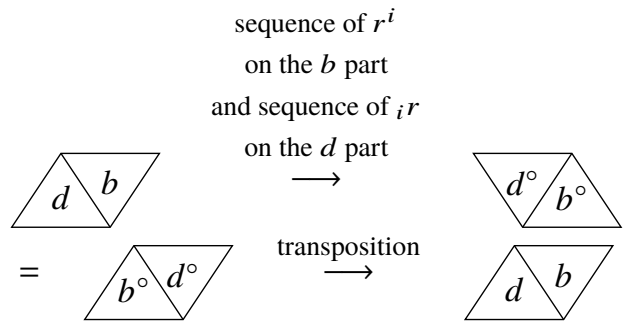

In conclusion, we have proved the following theorem.

Theorem 4.8. The cluster Donaldson-Thomas transformation on $\operatorname{Conf}_{d}^{b}(\mathcal{B})$ is the composition of a series of reflection maps on both sides and the transposition map as described above.

Remark 4.9. When we computed $\mathrm{DT}_{\operatorname{Conf}_{d}^{b}(\mathcal{B})}=\eta^{-1} \circ \mathrm{DT}_{\mathrm{Conf}_{d b^{\circ}}^{e}(\mathcal{B})} \circ \eta$ we chose to work with the cluster transformation $\eta: \operatorname{Conf}_{d}^{b}(\mathcal{B}) \rightarrow \operatorname{Conf}_{d b^{\circ}}^{e}(\mathcal{B})$ defined as a composition of reflections $r^{i}$; alternatively, one can work with the cluster transformation $\eta^{\prime}: \operatorname{Conf}_{d}^{b}(\mathcal{B}) \rightarrow \operatorname{Conf}_{b^{\circ} d}^{e}(\mathcal{B})$ defined as a composition of reflections ${ }^{i} r$. The resulting cluster Donaldson-Thomas transformation $\mathrm{DT}_{\mathrm{Conf}_{d}^{b}(\mathcal{B})}$ would be the same due to the uniqueness of cluster Donaldson-Thomas transformation. Moreover, we could have studied $\mathrm{DT}_{\mathrm{Conf}_{e}^{b}(\mathcal{B})}$ instead of $\mathrm{DT}_{\mathrm{Conf}_{b}^{e}(\mathcal{B})}$ and used it to compute the general case; the result would also be the same.

The existence of the cluster Donaldson-Thomas transformation is a part of a sufficient condition (Theorem A.38) of the Fock-Goncharov cluster duality conjecture (Conjecture A.26). In the cluster ensemble $\left(\operatorname{Conf}_{d}^{b}\left(\mathcal{A}_{\mathrm{sc}}\right), \operatorname{Conf}_{d}^{b}\left(\mathcal{A}_{\mathrm{ad}}\right)\right)$, we already proved the surjectivity of the map $p: \operatorname{Conf}_{d}^{b}\left(\mathcal{A}_{\mathrm{sc}}\right) \rightarrow$ $\operatorname{Conf}_{d}^{b}(\mathcal{B})$ from the proof of Theorem 3.45 and we just constructed the cluster Donaldson-Thomas transformation explicitly on $\operatorname{Conf}_{d}^{b}(\mathcal{B})$. Therefore, we obtain the following result.

Theorem 4.10. The Fock-Goncharov cluster duality conjecture A.26 holds for the cluster ensemble $\left(\operatorname{Conf}_{d}^{b}\left(\mathcal{A}_{\mathrm{sc}}\right), \operatorname{Conf}_{d}^{b}\left(\mathcal{A}_{\mathrm{ad}}\right)\right)$.

\subsection{Reflection Maps as Quasi-Cluster Transformations}

So far we have considered the Donaldson-Thomas transformation on the undecorated double BottSamelson cell $\operatorname{Conf}_{d}^{b}(\mathcal{B})$, which can be seen as the unfrozen part of a cluster Poisson variety $\mathscr{X}_{d}^{b}$ (up to codimension 2). In this section we would like to investigate the lift of the Donaldson-Thomas transformation to the decorated double Bott-Samelson cell $\operatorname{Conf}_{d}^{b}(\mathcal{A})$. Because the Donaldson-Thomas 
transformation on $\operatorname{Conf}_{d}^{b}(\mathcal{B})$ can be broken down into a sequence of reflection maps followed by a transposition, we can define the lift of the Donaldson-Thomas transformation to $\operatorname{Conf}_{d}^{b}(\mathcal{B})$ to $\operatorname{Conf}_{d}^{b}(\mathcal{A})$ to be the same composition of reflection maps and transposition on $\operatorname{Conf}_{d}^{b}(\mathcal{A})$ according to Subsection 2.3.

The next question is whether such a lift is a cluster transformation. Unfortunately, the answer is no: in general, the reflection maps between decorated double Bott-Samelson cells are not cluster isomorphisms because the underlying seeds are not isomorphic. However, the following weaker statement is true (see Definition A.32 for the definition of quasi-cluster transformation).

\section{Proposition 4.11. Reflection maps are quasi-cluster transformations.}

Proof. Because left and right reflection maps are intertwined by a transposition map, which is a cluster isomorphism even on the decorated double Bott-Samelson cell level, it suffices to only show the claim for right reflection maps. But then because $r^{i}$ and $r_{i}$ are inverses of each other and the inverse of a quasi-cluster transformation is also a quasi-cluster transformation, it suffices to only consider $r^{i}$.

Let us first consider the adjoint case $r^{i}: \operatorname{Conf}_{d}^{b s_{i}}\left(\mathcal{A}_{\mathrm{ad}}\right) \rightarrow \operatorname{Conf}_{d s_{i}}^{b}\left(\mathcal{A}_{\mathrm{ad}}\right)$. Suppose we start with the following cluster coordinate chart. Then $r^{i}$ should produce the last configuration according to its definition.
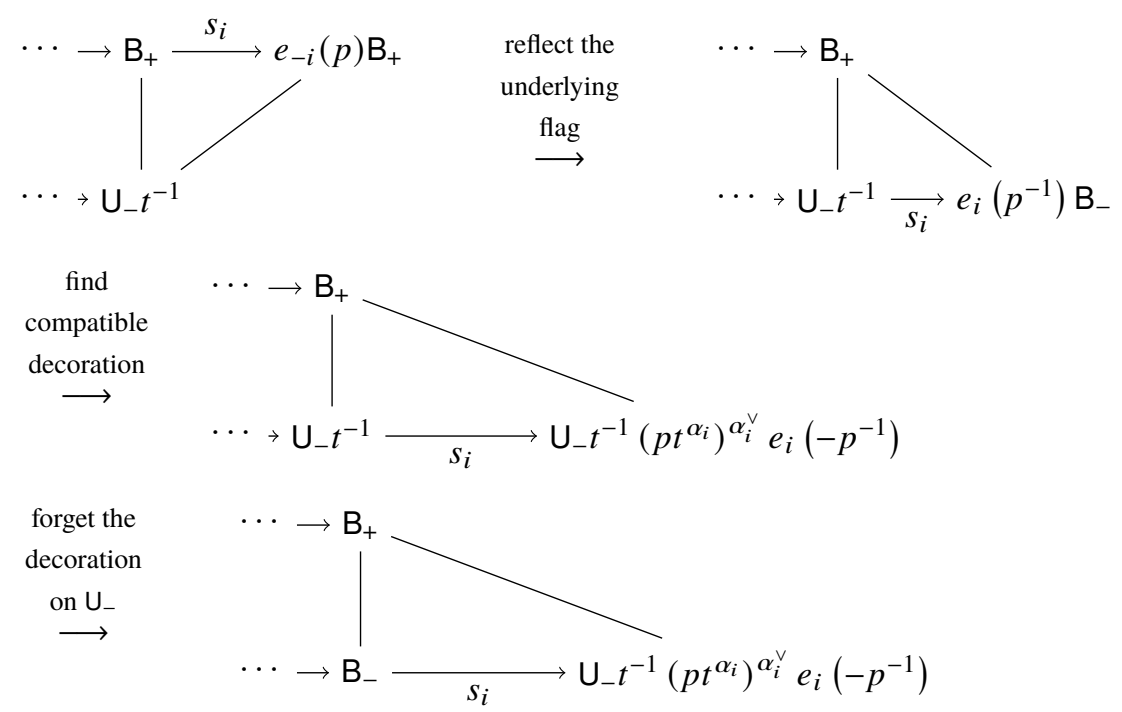

According to the construction of the Lusztig factorisation coordinates and the cluster Poisson coordinates, the last picture above indicates that the Lusztig factorisation corresponding to the given triangulation before $r^{i}$ ends at $\ldots e_{-i}(p) t$, which corresponds to the following cluster Poisson coordinates; note that the dots represent the factors that possibly come from the other ends of the strings (note that the squiggly arrow only denotes the sign of the exchange matrix).

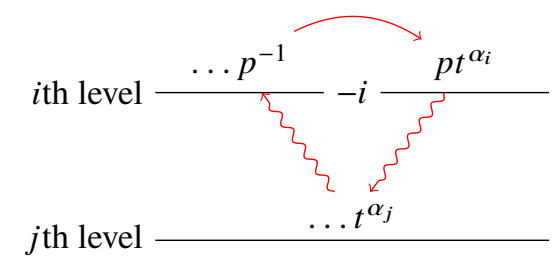


After applying $r^{i}$, the Lusztig factorisation ends at $\ldots e_{i}\left(p^{-1}\right) t\left(p t^{\alpha_{i}}\right)^{-\alpha_{i}^{\vee}}$, which corresponds to the following cluster Poisson coordinates:

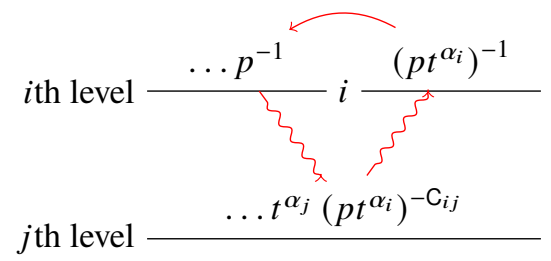

By comparing the two, we see that the pullback of most cluster Poisson coordinates under $r^{i}$ remains unchanged; the only cluster Poisson coordinates that are different are the ones associated to the open strings on the right side of the string diagram. In particular, the last frozen variable $X_{i}$ on the $i$ th level gets inverted:

$$
r^{i *}\left(X_{i}^{\prime}\right)=X_{i}^{-1}
$$

and the last frozen variable $X_{j}$ on any other level (say $j$ th) is rescaled:

$$
r^{i *}\left(X_{j}^{\prime}\right)=X_{j} X_{i}^{-C_{i j}}
$$

Because $\left.r^{i}\right|_{\operatorname{Conf}_{d}^{b s_{i}(\mathcal{B})}}$ is already known to be a cluster transformation, to show that $r^{i}: \operatorname{Conf}_{d}^{b s_{i}}\left(\mathcal{A}_{\mathrm{ad}}\right) \rightarrow$ $\operatorname{Conf}_{d s_{i}}^{b}\left(\mathcal{A}_{\text {ad }}\right)$ is a quasi-cluster transformation, we only need to show that $r^{i}$ is a Poisson map, which boils down to showing

$$
\left\{r^{i *} X_{a}^{\prime}, r^{i *} X_{b}^{\prime}\right\}=\left\{X_{a}^{\prime}, X_{b}^{\prime}\right\}
$$

for any two strings $a$ and $b$. From the string diagram of $\operatorname{Conf}_{d}^{b s_{i}}\left(\mathcal{A}_{\mathrm{ad}}\right)$ we see that $X_{i}$ only has nonvanishing Poisson bracket with the variable associated to the closed string to the left of it (call it $X_{c}$ ) and the frozen variables $X_{j}$ on the other levels. Therefore, any change to Poisson brackets of cluster Poisson variables can only occur on $\left\{X_{c}^{\prime}, X_{i}^{\prime}\right\},\left\{X_{c}^{\prime}, X_{j}^{\prime}\right\}$ and $\left\{X_{i}^{\prime}, X_{j}^{\prime}\right\}$. Because the Poisson structure on a cluster Poisson variety is known to be log canonical, we apply log to simplify the computation:

$$
\begin{aligned}
\left\{\log \left(r^{i *} X_{c}^{\prime}\right), \log \left(r^{i *} X_{i}^{\prime}\right)\right\} & =\left\{\log X_{c}, \log X_{i}^{-1}\right\}=-\left\{\log X_{c}, \log X_{i}\right\}=-\frac{1}{\mathrm{D}_{i}} \\
& =\left\{\log X_{c}^{\prime}, \log X_{i}^{\prime}\right\} \\
\left\{\log \left(r^{i *} X_{c}^{\prime}\right), \log \left(r^{i *} X_{j}^{\prime}\right)\right\} & =\left\{\log X_{c}, \log \left(X_{j} X_{i}^{-C_{i j}}\right)\right\} \\
& =\left\{\log X_{c}, \log X_{j}\right\}-\mathrm{C}_{i j}\left\{\log X_{c}, \log X_{i}\right\} \\
& =\frac{\mathrm{C}_{j i}}{2 \mathrm{D}_{j}}-\frac{\mathrm{C}_{i j}}{\mathrm{D}_{i}}+\mathrm{E}=\frac{\mathrm{C}_{j i}}{2 \mathrm{D}_{j}}-\frac{\mathrm{C}_{j i}}{\mathrm{D}_{j}}+\mathrm{E}=-\frac{\mathrm{C}_{j i}}{2 \mathrm{D}_{j}}+\mathrm{E} \\
& =\left\{\log X_{c}^{\prime}, \log X_{j}^{\prime}\right\} \\
\left\{\log \left(r^{i *} X_{i}^{\prime}\right), \log \left(r^{i *} X_{j}^{\prime}\right)\right\} & =\left\{\log X_{i}^{-1}, \log \left(X_{j} X_{i}^{-\mathrm{C}_{i j}}\right)\right\}=-\left\{\log X_{i}, \log X_{j}\right\} \\
& =-\left(-\frac{\mathrm{C}_{j i}}{\mathrm{D}_{j}}\right)=\frac{\mathrm{C}_{j i}}{\mathrm{D}_{j}}=\left\{\log X_{i}^{\prime}, \log X_{j}^{\prime}\right\} .
\end{aligned}
$$

Here $\mathrm{E}$ accounts for the contribution from the earlier parts of the diagram and they are invariant under right reflections. This finishes proving that the reflection map $r^{i}$ is a quasi-cluster transformation in the adjoint case. 
Next let us consider the simply connected case. By definition, the action of a quasi-cluster transformation on a cluster $\mathrm{K}_{2}$ variety is induced from its action on a cluster Poisson variety. If we express the action of $r^{i}$ on our chosen seed above using the character lattice $N$ of $T_{\mathscr{X}}$, it can be expressed as the following matrix, which consists of an identity matrix for the most part except for the submatrix with rows and columns corresponding to the frozen variables on the right:

$$
\left(\begin{array}{ccccc}
\mathrm{id} & 0 & 0 & \cdots & 0 \\
0 & -1 & 0 & \cdots & 0 \\
0 & -\mathrm{C}_{i j} & 1 & \cdots & 0 \\
\vdots & \vdots & \vdots & \ddots & 0 \\
0 & -\mathrm{C}_{i k} & 0 & \cdots & 1 \text { th } \\
j \text { th } & \vdots \\
k \text { th }
\end{array}\right.
$$

By Lemma A.33, the induced quasi-cluster transformation action on the cluster $\mathrm{K}_{2}$ variety should then be acting on the character lattice $M$ of $T_{\mathscr{A}}$ by the matrix that is the transpose inverse of the one above with $\mathrm{C}_{i j}$ replaced by $\mathrm{C}_{j i}$, which is

$$
\left(\begin{array}{ccccc}
\mathrm{id} & 0 & 0 & \cdots & 0 \\
0 & -1 & -\mathrm{C}_{j i} & \cdots & -\mathrm{C}_{k i} \\
0 & 0 & 1 & \cdots & 0 \\
\vdots & \vdots & \vdots & \ddots & 0 \\
0 & 0 & 0 & \cdots & 1
\end{array}\right) \begin{gathered}
j \text { th } \\
\vdots \\
k \text { th }
\end{gathered}
$$

Therefore, to show that $r^{i}: \operatorname{Conf}_{d}^{b s_{i}}\left(\mathcal{A}_{\mathrm{sc}}\right) \rightarrow \operatorname{Conf}_{d s_{i}}^{b}\left(\mathcal{A}_{\mathrm{sc}}\right)$ is a quasi-cluster transformation, it suffices to show that all cluster $\mathrm{K}_{2}$ coordinates remain the same except the last frozen variable $A_{i}^{\prime}$ on the $i$ th level, which should transform by

$$
r^{i *}\left(A_{i}^{\prime}\right)=A_{i}^{-1} \prod_{j \neq i} A_{j}^{-\mathrm{C}_{j i}}
$$

So let us verify this transformation formula geometrically. By a similar computation as in the adjoint case, the reflection map turns the following configuration on the left to the one on the right in the simply connected case:

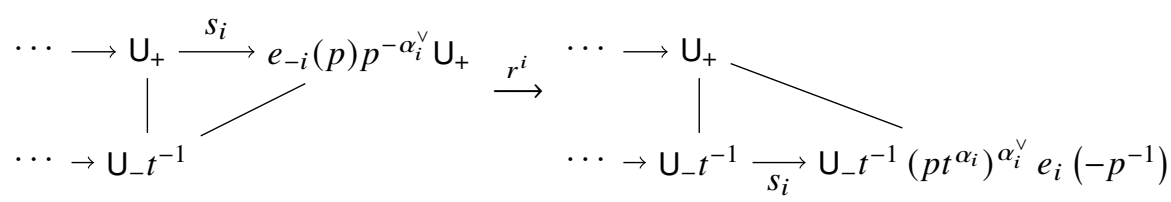

Because the cluster $\mathrm{K}_{2}$ coordinates are computed by evaluating the $\left(\mathrm{U}_{-}, \mathrm{U}_{+}\right)$-invariant function $\Delta_{\omega_{j}}$ and $\left\langle\alpha_{i}^{\vee}, \omega_{j}\right\rangle=\delta_{i j}$, we see that the only cluster $\mathrm{K}_{2}$ coordinate that changes under $r^{i}$ is $A_{i}^{\prime}$ :

$$
r^{i *}\left(A_{i}^{\prime}\right)=\Delta_{\omega_{i}}\left(t^{-1}\left(p t^{\alpha_{i}}\right)^{\alpha_{i}^{\vee}}\right)=p t^{\alpha_{i}-\omega_{i}}
$$

On the other hand, by computation we get

$$
A_{i}^{-1} \prod_{j \neq i} A_{j}^{-\mathrm{C}_{j i}}=\left(p^{-\alpha_{i}^{\vee}} t^{-1}\right)^{-\omega_{i}} \prod_{j \neq i}\left(\left(p^{-\alpha_{i}^{\vee}} t^{-1}\right)^{\omega_{j}}\right)^{-\mathrm{C}_{j i}}=p t^{\omega_{i}} t^{\sum_{j \neq i} \mathrm{C}_{j i} \omega_{j}}=p t^{\alpha_{i}-\omega_{i}}
$$

which agrees with $r^{i *}\left(A_{i}^{\prime}\right)$, as predicted by the transformation formula. 
Because reflection maps are quasi-cluster transformations on the decorated double Bott-Samelson cells and the transposition map is a cluster isomorphism, it follows that the Donaldson-Thomas transformation on decorated double Bott-Samelson cells, as a composition of such maps, is also a quasi-cluster transformation.

Corollary 4.12. The Donaldson-Thomas transformation on any decorated double Bott-Samelson cell is a quasi-cluster transformation. It then follows that the Donaldson-Thomas transformation on $\operatorname{Conf}_{d}^{b}\left(\mathcal{A}_{\mathrm{ad}}\right)$ is a Poisson map.

Using the fact that reflection maps are quasi-cluster transformations, we can also prove the following sufficient condition on the equality between upper cluster algebras and cluster algebras (Theorem 1.2).

Theorem 4.13. The upper cluster algebra $\mathcal{O}\left(\operatorname{Conf}_{d}^{b}\left(\mathcal{A}_{\mathrm{sc}}\right)\right)$ coincides with its cluster algebra.

Proof. By Theorem 3.45, it suffices to show that $\mathcal{O}\left(\operatorname{Conf}_{d}^{b}\left(\mathcal{A}_{\mathrm{sc}}\right)\right)$ is generated by cluster variables (with invertible frozen cluster variables). From Lemma A.33 we know that a quasi-cluster transformation modifies unfrozen cluster $\mathrm{K}_{2}$ variables by Laurent monomials in the frozen cluster $\mathrm{K}_{2}$ variables and maps the frozen cluster $\mathrm{K}_{2}$ variables to Laurent monomials in the frozen cluster $\mathrm{K}_{2}$ variables. Therefore, we can apply a sequence of reflection maps to reduce the question to proving that $\mathcal{O}\left(\operatorname{Conf}_{d}^{e}\left(\mathcal{A}_{\mathrm{sc}}\right)\right)$ is generated by cluster variables for any positive braid $d$.

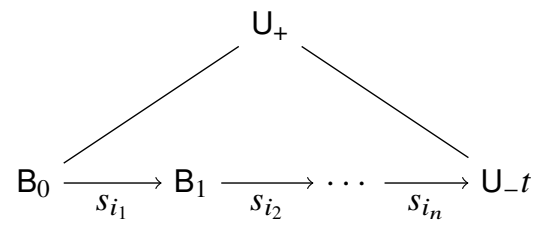

Recall from Theorem 2.30 that any point in $\operatorname{Conf}_{d}^{b}\left(\mathcal{A}_{\mathrm{sc}}\right)$ can be represented by a unique representative as shown above and $\operatorname{Conf}_{d}^{b}\left(\mathcal{A}_{\mathrm{sc}}\right)$ is the nonvanishing locus of a single function $f$ in $\mathrm{T} \times \mathbb{A}^{n}$, where $\mathrm{T}$ captures the freedom of the maximal torus element $t$ and the coordinates of $\mathbb{A}^{n}$ are parametrising the relative positions between each pair of adjacent flags in the bottom chain. Note that by definition, the function $f$ is the product of the frozen cluster $\mathrm{K}_{2}$ variables on the left and the torus factor $\mathrm{T}$ is parametrised by the frozen cluster $\mathrm{K}_{2}$ variables on the right. By assumption, the frozen cluster $\mathrm{K}_{2}$ variables are invertible in the cluster algebra, too. Therefore, it remains to show that the coordinates of the affine space factor $\mathbb{A}^{n}$ are polynomials in unfrozen cluster $\mathrm{K}_{2}$ variables and Laurent polynomials in frozen cluster $\mathrm{K}_{2}$ variables.

First, from the proof of Lemma 3.30 we know that the first affine parameter of the pair $\mathrm{B}_{0} \stackrel{s_{i_{1}}}{\longrightarrow} \mathrm{B}_{1}$ is a monomial in unfrozen cluster $\mathrm{K}_{2}$ variables and a Laurent monomial in frozen cluster $\mathrm{K}_{2}$ variables. Next we can apply the quasi-cluster transformation $r^{i_{1}}{ }^{\circ}{ }_{i_{1}} r$ to move first letter $s_{i_{1}}$ to the end of the $d$ chain. Then Lemma 3.30 implies again that the affine parameter of the pair $\mathrm{B}_{1} \stackrel{s_{i_{2}}}{\longrightarrow} \mathrm{B}_{2}$ is a monomial in unfrozen cluster $\mathrm{K}_{2}$ variables (not necessarily of the initial seed) and a Laurent monomial in frozen cluster $\mathrm{K}_{2}$ variables. The same argument can be applied to the rest of the affine parameters and we can conclude that $\mathcal{O}\left(\operatorname{Conf}_{d}^{e}\left(\mathcal{A}_{\mathrm{sc}}\right)\right)$ is generated by cluster $\mathrm{K}_{2}$ variables (with invertible frozen variables).

\section{Periodicity of DT transformations and Zamolodchikov's Periodicity Conjecture}

In this section we prove the periodicity of DT transformations for a family of double Bott-Samelson cells. We give a new geometric proof of Zamolodchikov's periodicity conjecture.

Below $\mathrm{G}$ is a semisimple algebraic group. The longest Weyl group element $w_{0}$ can be uniquely lifted to a positive braid. Its square $\Omega:=w_{0}^{2}$ is a central element in the braid group $\operatorname{Br}$. Let $b=s_{i_{1}} s_{i_{2}} \ldots s_{i_{n}}$ be a positive braid. Recall that $b^{\circ}=s_{i_{n}} \ldots s_{i_{2}} s_{i_{1}}$. 
Theorem 5.1. Let $b$ and $d$ be positive braids such that $\left(d b^{\circ}\right)^{m}=\Omega^{n}$. The cluster Donaldson-Thomas transformation of $\operatorname{Conf}_{d}^{b}(\mathcal{B})$ is of a finite order dividing $2(m+n)$.

Example 5.2. Let $\mathrm{G}=\mathrm{PGL}_{7}$. Its Weyl group is the symmetric group $S_{7}$. Take the element $u=$ (1 23765$) \in S_{7}$ in cycle notation. The length of $u$ is 7. Its lift to Br satisfies $u^{3}=w_{0}$. Let $d=u^{n} \in \mathrm{Br}$. Then $d^{6}=\Omega^{n}$. The order of the DT transformation of $\operatorname{Conf}_{d}^{e}(\mathcal{B})$ divides $12+2 n$.

Proof. The cluster Donaldson-Thomas transformations on $\operatorname{Conf}_{d}^{b}(\mathcal{B})$ and $\operatorname{Conf}_{d b^{\circ}}^{e}(\mathcal{B})$ are intertwined by a sequence of reflection maps on the right; hence, they share the same order. It suffices to consider the cases $\operatorname{Conf}_{b}^{e}(\mathcal{B})$ with $b^{m}=\Omega^{n}$. Let $b=s_{i_{1}} s_{i_{2}} \ldots s_{i_{l}}$. We start with a configuration

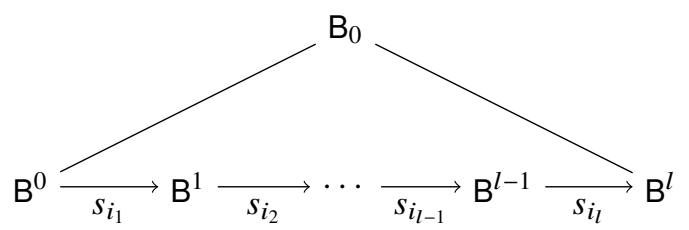

The cluster DT transformation of $\operatorname{Conf}_{b}^{e}(\mathcal{B})$ is a sequence of left reflections followed by transposition. Concretely, we obtain a collection of Borel subgroups $\mathrm{B}_{1}, \ldots, \mathrm{B}_{l}$ by the reflections ${ }^{i} r$

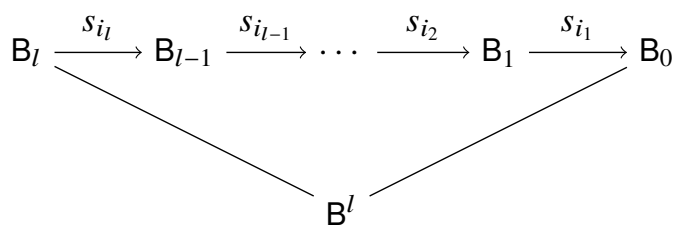

and then apply the transposition map to get a new configuration in $\operatorname{Conf}_{b}^{e}(\mathcal{B})$

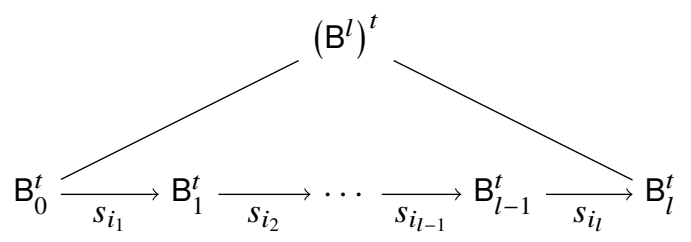

Let us apply the cluster DT transformation to (5.4) again, obtaining

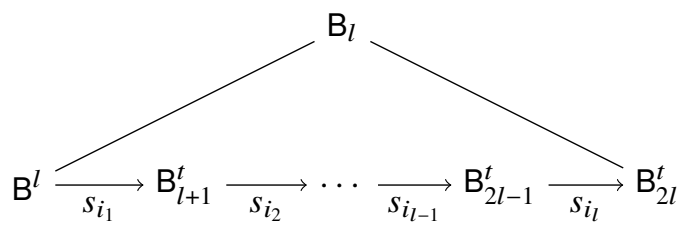

To better describe the patterns, we introduce the following notations for Borel subgroups in $\mathcal{B}_{+}$:

$$
\mathrm{B}_{(0)}:=\tau\left(\mathrm{B}^{0}\right), \quad \mathrm{B}_{(1)}:=\mathrm{B}_{0}, \quad \mathrm{~B}_{(2)}:=\tau\left(\mathrm{B}^{l}\right), \quad \mathrm{B}_{(3)}:=\mathrm{B}_{l}, \quad \mathrm{~B}_{(4)}:=\mathrm{B}_{2 l},
$$

where $\tau$ is a natural isomorphism from $\mathcal{B}_{-}$to $\mathcal{B}_{+}$that takes $\mathrm{B}_{-} g$ to $g^{-1} \bar{w}_{0} \mathrm{~B}_{+}$. Recall the automorphism $*$ on $\mathcal{B}_{+}$by $\mathrm{B} \mapsto \mathrm{B}^{*}:=\tau\left(\mathrm{B}^{t}\right)$. The configurations (5.3)-(5.5) can be rewritten as 


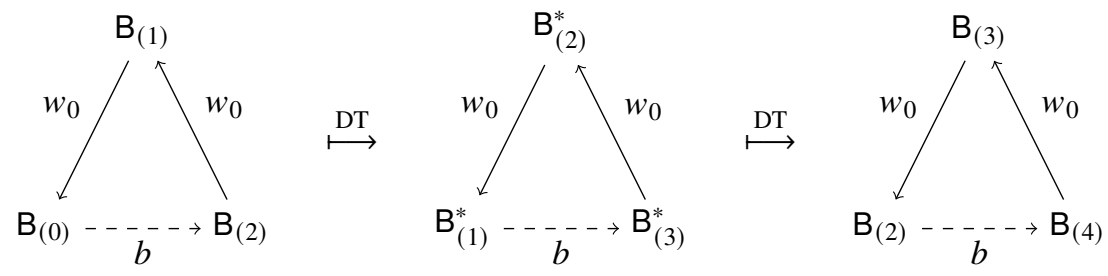

Here in each configuration we abbreviate the bottom $b$-chain into a single dashed arrow. We adopt the same convention in the rest of the proof.

Let us cut the first and last triangles in (5.6) at $\mathrm{B}_{(2)}$. We claim that the obtained chains

$$
\mathrm{B}_{(2)} \stackrel{w_{0}}{\longrightarrow} \mathrm{B}_{(1)} \stackrel{w_{0}}{\longrightarrow} \mathrm{B}_{(0)}-\stackrel{b}{-}>\mathrm{B}_{(2)}, \quad \mathrm{B}_{(2)}-\stackrel{b}{-}>\mathrm{B}_{(4)} \stackrel{w_{0}}{\longrightarrow} \mathrm{B}_{(3)} \stackrel{w_{0}}{\longrightarrow} \mathrm{B}_{(2)}
$$

are equivalent under the braid moves from $\Omega b$ to $b \Omega$. Indeed, let $\mathrm{B}_{1}$ be the unique flag such that $\mathrm{B}_{(1)} \stackrel{s_{i_{1}}^{*}}{\longrightarrow} \mathrm{B}_{1} \stackrel{w_{0} s_{i_{1}}}{\longrightarrow} \mathrm{B}_{(0)}$. The first left reflection in the first DT is

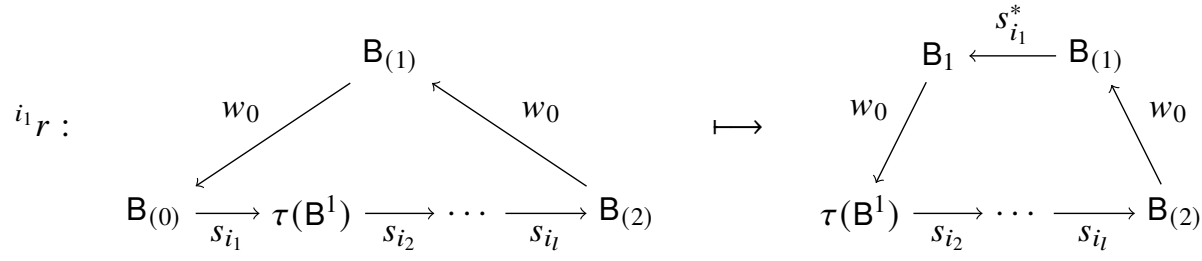

Let us cut them at $\mathrm{B}_{(2)}$. The obtained chains are equivalent under the braid moves $w_{0} s_{i_{1}}=s_{i_{1}}^{*} w_{0}$ :

$$
\begin{aligned}
& \mathrm{B}_{(2)} \stackrel{w_{0}}{\longrightarrow} \mathrm{B}_{(1)} \stackrel{w_{0}}{\longrightarrow} \mathrm{B}_{(0)}-\stackrel{b}{\longrightarrow}>\mathrm{B}_{(2)} \\
= & \mathrm{B}_{(2)} \stackrel{w_{0}}{\longrightarrow} \mathrm{B}_{(1)} \stackrel{w_{0}}{\longrightarrow} \mathrm{B}_{(0)} \stackrel{s_{i_{1}}}{\longrightarrow} \tau\left(\mathrm{B}^{1}\right) \stackrel{s_{i_{1}}^{-1} b}{-}>\mathrm{B}_{(2)} \\
= & \mathrm{B}_{(2)} \stackrel{w_{0}}{\longrightarrow} \mathrm{B}_{(1)} \stackrel{s_{i_{1}}^{*}}{\longrightarrow} \mathrm{B}_{1} \stackrel{w_{0}}{\longrightarrow} \tau\left(\mathrm{B}^{1}\right) \stackrel{s_{i_{1}}^{-1} b}{-}>\mathrm{B}_{(2)}
\end{aligned}
$$

Repeat the same procedure for the rest of the left reflections. Eventually the braid moves from $w_{0} b$ to $b^{*} w_{0}$ turn the chain $\mathrm{B}_{(2)} \stackrel{w_{0}}{\longrightarrow} \mathrm{B}_{(1)} \stackrel{w_{0}}{\longrightarrow} \mathrm{B}_{(0)}-\stackrel{b}{\rightarrow} \mathrm{B}_{(2)}$ into $\mathrm{B}_{(2)} \stackrel{w_{0}}{\longrightarrow} \mathrm{B}_{(1)}-\stackrel{b^{*}}{\rightarrow} \mathrm{B}_{(3)} \stackrel{w_{0}}{\longrightarrow} \mathrm{B}_{(2)}$. Similarly, the second DT turns the latter into $\mathrm{B}_{(2)}-\stackrel{b}{>} \mathrm{B}_{(4)} \stackrel{w_{0}}{\longrightarrow} \mathrm{B}_{(3)} \stackrel{w_{0}}{\longrightarrow} \mathrm{B}_{(2)}$ by braid moves.

Applying the transformation $\mathrm{DT}^{2}$ recursively, we obtain the configurations

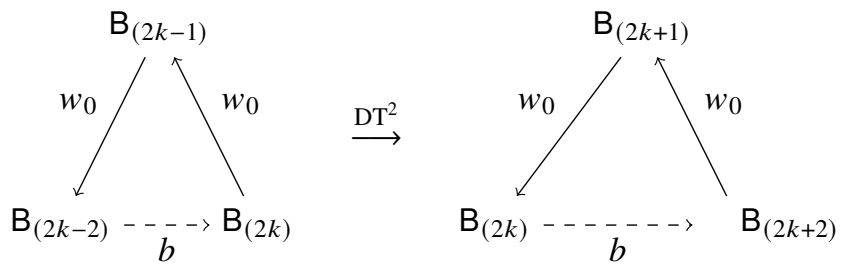

Let us concatenate $\max \{m, n\}+1$ copies of the chain $\mathrm{B}_{(2 m)} \stackrel{b}{\rightarrow} \mathrm{B}_{(2 m+2)} \stackrel{w_{0}}{\rightarrow} \mathrm{B}_{(2 m+1)} \stackrel{w_{0}}{\rightarrow} \mathrm{B}_{(2 m)}$. We apply $\mathrm{DT}^{2}$ to move $\Omega=w_{0}^{2}$ to the right. The braid moves $(b \Omega)^{n+1}=b^{n+1} \Omega^{1+n}$ give rise to 


$$
\begin{aligned}
& \ldots-\stackrel{b}{\rightarrow} \mathrm{B}_{(2 m+2)} \stackrel{w_{0}}{\rightarrow} \mathrm{B}_{(2 m+1)} \stackrel{w_{0}}{\rightarrow} \mathrm{B}_{(2 m)} \stackrel{b}{\rightarrow} \mathrm{B}_{(2 m+2)} \stackrel{w_{0}}{\rightarrow} \mathrm{B}_{(2 m+1)} \stackrel{w_{0}}{\rightarrow} \mathrm{B}_{(2 m)} \\
= & \ldots \stackrel{w_{0}}{\rightarrow} \mathrm{B}_{(2 m+2)} \stackrel{b}{\rightarrow} \mathrm{B}_{(2 m+4)} \stackrel{w_{0}}{\rightarrow} \mathrm{B}_{(2 m+3)} \stackrel{w_{0}}{\rightarrow} \mathrm{B}_{(2 m+2)} \stackrel{w_{0}}{\rightarrow} \mathrm{B}_{(2 m+1))} \stackrel{w_{0}}{\rightarrow} \mathrm{B}_{(2 m)} \\
= & \ldots \\
= & \ldots \stackrel{b}{\rightarrow} \mathrm{B}_{(2(m+n))} \stackrel{b}{\rightarrow} \mathrm{B}_{(2(m+n)+2)} \stackrel{w_{0}}{\rightarrow} \mathrm{B}_{(2(m+n)+1)} \stackrel{w_{0}}{\rightarrow} \mathrm{B}_{(2(m+n))} \stackrel{\Omega^{n}}{\rightarrow} \mathrm{B}_{(2 m)} .
\end{aligned}
$$

Conversely, we apply $\mathrm{DT}^{-2}$ to move $b$ to the right and obtain

$$
\begin{aligned}
& \ldots \stackrel{b}{\rightarrow} \mathrm{B}_{(2 m+2)} \stackrel{w_{0}}{\rightarrow} \mathrm{B}_{(2 m+1)} \stackrel{w_{0}}{\rightarrow} \mathrm{B}_{(2 m)} \stackrel{b}{\rightarrow} \mathrm{B}_{(2 m+2)} \stackrel{w_{0}}{\rightarrow} \mathrm{B}_{(2 m+1)} \stackrel{w_{0}}{\rightarrow} \mathrm{B}_{(2 m)} \\
= & \ldots \stackrel{w_{0}}{\rightarrow} \mathrm{B}_{(2 m-1)} \stackrel{w_{0}}{\rightarrow} \mathrm{B}_{(2 m-2)} \stackrel{b}{\rightarrow} \mathrm{B}_{(2 m)} \stackrel{w_{0}}{\rightarrow} \mathrm{B}_{(2 m-1)} \stackrel{w_{0}}{\rightarrow} \mathrm{B}_{(2 m-2)} \stackrel{b}{\rightarrow} \mathrm{B}_{(2 m)} \\
= & \ldots \stackrel{w_{0}}{\rightarrow} \mathrm{B}_{(2 m-4)} \stackrel{b}{\rightarrow} \mathrm{B}_{(2 m-2)} \stackrel{w_{0}}{\rightarrow} \mathrm{B}_{(2 m-3)} \stackrel{w_{0}}{\rightarrow} \mathrm{B}_{(2 m-4)} \stackrel{b}{\rightarrow} \mathrm{B}_{(2 m-2)} \stackrel{b}{\rightarrow} \mathrm{B}_{(2 m)} \\
= & \ldots \\
= & \ldots \stackrel{w_{0}}{\rightarrow} \mathrm{B}_{(0)} \stackrel{b}{\rightarrow} \mathrm{B}_{(2)} \stackrel{w_{0}}{\rightarrow} \mathrm{B}_{(1)} \stackrel{w_{0}}{\rightarrow} \mathrm{B}_{(0)} \stackrel{b}{\rightarrow} \mathrm{B}_{(2)} \stackrel{b}{\rightarrow} \mathrm{B}_{(4)} \stackrel{b}{\rightarrow} \ldots \stackrel{b}{\rightarrow} \mathrm{B}_{(2 m)} \\
= & \ldots \stackrel{w_{0}}{\rightarrow} \mathrm{B}_{(0)} \stackrel{b}{\rightarrow} \mathrm{B}_{(2)} \stackrel{w_{0}}{\rightarrow} \mathrm{B}_{(1)} \stackrel{w_{0}}{\rightarrow} \mathrm{B}_{(0)} \stackrel{\Omega^{n}}{\rightarrow} \mathrm{B}_{(2 m)} .
\end{aligned}
$$

Here the last step uses the condition that $b^{m}=\Omega^{n}$.

Let us compare the two final chains under the above braid moves. By Theorem 2.18, we get

$$
\begin{aligned}
& \mathrm{B}_{(0)} \stackrel{b}{\rightarrow} \mathrm{B}_{(2)} \stackrel{w_{0}}{\rightarrow} \mathrm{B}_{(1)} \stackrel{w_{0}}{\rightarrow} \mathrm{B}_{(0)} \\
& =\mathrm{B}_{(2(m+n))} \stackrel{b}{\rightarrow} \mathrm{B}_{(2(m+n)+2)} \stackrel{w_{0}}{\rightarrow} \mathrm{B}_{(2(m+n)+1)} \stackrel{w_{0}}{\rightarrow} \mathrm{B}_{(2(m+n))},
\end{aligned}
$$

which concludes the proof.

\section{Zamolodchikov's periodicity conjecture}

A $Y$-system is a system of algebraic recurrence equations associated with a pair of Dynkin diagrams. The periodicity conjecture, first formulated by Zamolodchikov in his study of thermodynamic Bethe ansatz, asserts that all solutions to this system are of period dividing the double of the sum of the Coxeter numbers of underlying Dynkin diagrams. This periodicity property plays a significant role in conformal field theory and statistical mechanics. It was first settled by Keller [Kel13] in full generality by using highly nontrivial techniques including cluster algebras and their additive categorification. We refer to [Kel13] for an introduction to the periodicity conjecture.

Let us reformulate the periodicity conjecture in terms of cluster mutations. Let $\Delta$ be a Dynkin diagram with the Cartan matrix $\mathrm{C}$. A bipartite colouring on $\Delta$ gives rise to a seed with the same vertex set $I$, multipliers $\left\{d_{a}\right\}$ and the exchange matrix

$$
\epsilon_{a b}= \begin{cases}c(a) \mathrm{C}_{b a} & \text { if } a \neq b \\ 0 & \text { otherwise }\end{cases}
$$

where $c(a)=1$ if the vertex $a$ is coloured black and $c(a)=-1$ if it is coloured white. 
Given two bipartite Dynkin diagrams $\Delta$ and $\Delta^{\prime}$, their square product $\Delta \square \Delta^{\prime}$ is defined to be a seed with vertex set $I \times I^{\prime}$ and exchange matrix

$$
\epsilon_{\left(i, i^{\prime}\right),\left(j, j^{\prime}\right)}= \begin{cases}-c\left(i^{\prime}\right) \epsilon_{i j} & \text { if } i^{\prime}=j^{\prime} \\ c(i) \epsilon_{i^{\prime} j^{\prime}} & \text { if } i=j \\ 0 & \text { otherwise. }\end{cases}
$$

Example 5.7. The quiver $D_{4} \square A_{3}$ is as follows:

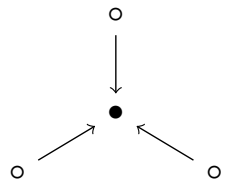

$\mathrm{D}_{4}$

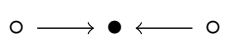

$\mathrm{A}_{3}$

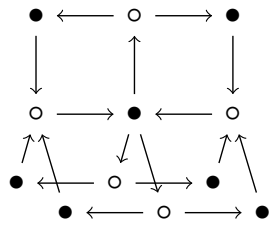

$\mathrm{D}_{4} \square \mathrm{A}_{3}$

Let $\tau=\tau_{-} \circ \tau_{+}$, where $\tau_{+}$is a sequence of mutations at the black vertices of $\Delta \square \Delta^{\prime}$ and $\tau_{-}$is a sequence of mutations at white ones. Note that $\tau$ preserves the quiver $\Delta \square \Delta^{\prime}$. Following [FZ03], the mutation sequence $\tau$ gives rise to the Zamolodchikov transformation Za on the cluster Poisson variety $\mathcal{X}_{\Delta \square \Delta^{\prime}}$. Let $h$ and $h^{\prime}$ be the Coxeter numbers of $\Delta$ and $\Delta^{\prime}$, respectively. By [Kel13, Lemma 2.4], Zamolodchikov's periodicity conjecture is equivalent to the identity

$$
\mathrm{Za}^{h+h^{\prime}}=\mathrm{Id}
$$

Below we give a new geometric proof of (5.8) for $\Delta \square \mathrm{A}_{n}$. Let $\mathrm{G}$ be a group of type $\Delta$ and let $\mathcal{B}$ be its flag variety. We fix a bipartite colouring on $\Delta$ and set

$$
b:=\underbrace{s_{b_{1}} s_{b_{2}} \ldots s_{b_{l}}}_{\text {black vertices }} \quad \text { and } \quad w:=\underbrace{s_{w_{1}} s_{w_{2}} \ldots s_{w_{m}}}_{\text {white vertices }} .
$$

Let

$$
p=\underbrace{w b w \ldots}_{n+1 \text { factors }} \text { and } q=\underbrace{b w b \ldots}_{n+1 \text { factors }}
$$

Recall the construction of seeds for double Bott-Samelson cells. The quiver associated to the following triangulation of $\operatorname{Conf}_{q}^{p}(\mathcal{B})$ is $\Delta \square \mathrm{A}_{n}$.

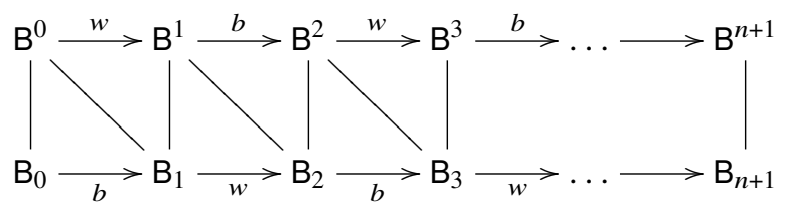

Therefore, $\operatorname{Conf}_{q}^{p}(\mathcal{B})$ is birationally isomorphic to the cluster Poisson variety $\mathcal{X}_{\Delta \square \mathrm{A}_{n}}$. 
Lemma 5.9. The transformation $\mathrm{Za}$ acts on $\operatorname{Conf}_{q}^{p}(\mathcal{B})$ as

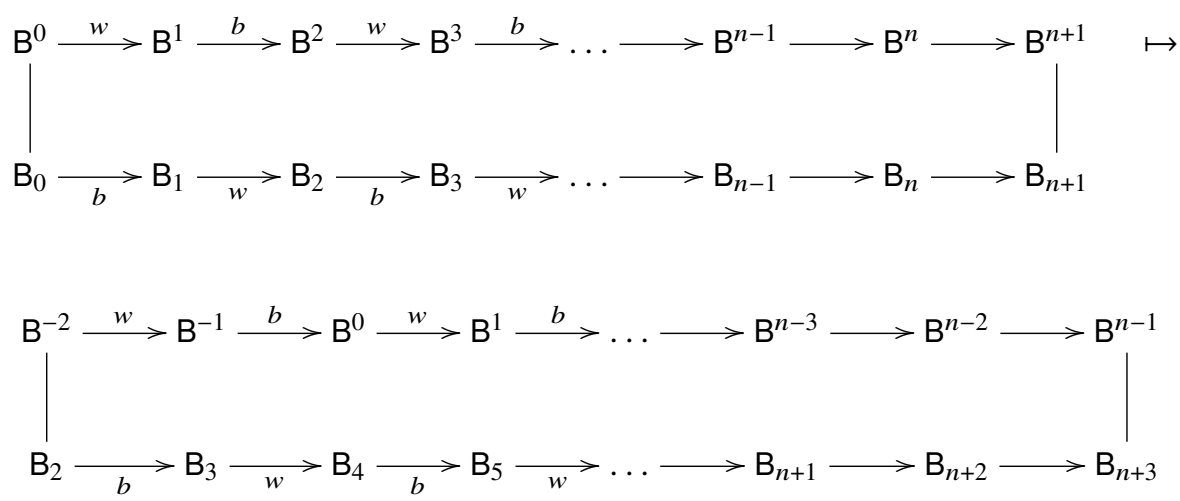

where $\mathrm{B}^{-2}$ and $\mathrm{B}^{-1}$ are the unique flags obtained by reflecting $\mathrm{B}_{1}$ and $\mathrm{B}_{0}$ on the left and $\mathrm{B}_{n+2}$ and $\mathrm{B}_{n+3}$ are obtained by reflecting $\mathrm{B}^{n+1}$ and $\mathrm{B}^{n}$ on the right. In other words, $\mathrm{Za}$ is equivalent to the composition of a Coxeter sequence of reflections on the left from bottom to top with another Coxeter sequence of reflections on the right from top to bottom.

Proof. The colours of the closed strings (vertices of the seed $\Delta \square \mathrm{A}_{n}$ ) look like the following:

white vertices in $\Delta$

black vertices in $\Delta$

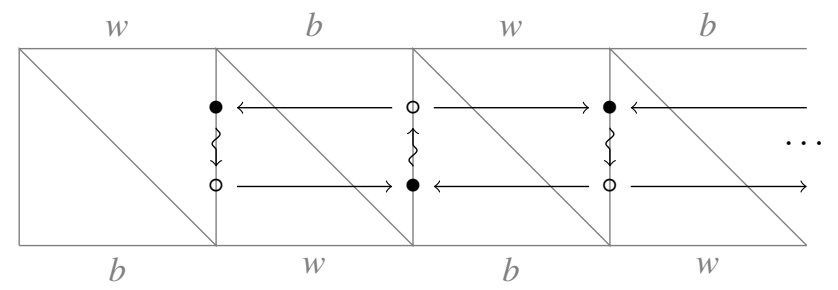

Note that black vertices in the quiver $\Delta \square \mathrm{A}_{n}$ correspond to strings cut out by triangles with the same base labellings. Mutations at all of the black vertices of $\Delta \square \mathrm{A}_{n}$ correspond to a collection of diagonal flips within a quadrilateral with the same base labellings (either $b$ or $w$ ). This turns the above triangulation into the left one below:
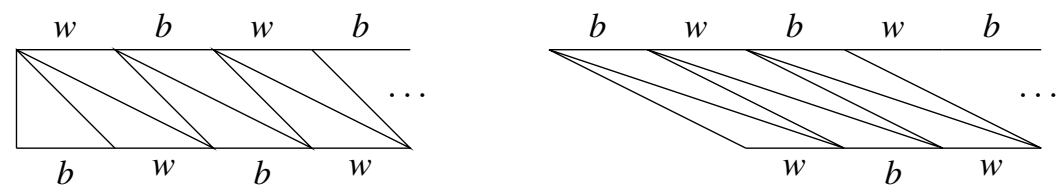

Now reflect the leftmost collection of triangles with base labelled by a $b$-chain to the top and the rightmost collection of triangles (with base labelled by either a $b$-chain or a $w$-chain) to the bottom and then flip the rest of the nonflipped diagonals. The resulting triangulation is depicted by the above right picture. Because these reflections and the diagonal flips only involve triangles that are labelled differently, no mutation is involved. By shifting the top chain to the right by two units we get the following triangulation on the left:
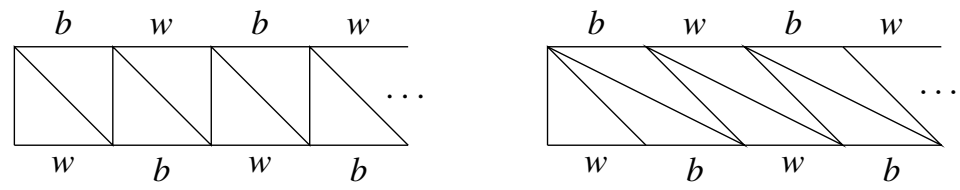
Similarly, mutating at the white vertices of $\Delta \square \mathrm{A}_{n}$ corresponds to another collection of diagonal flips, which turns the above left triangulation to the above right one. Let us again reflect the leftmost collection of triangles to the top and the rightmost collection of triangles to the bottom and flip the rest of the diagonals. The resulting triangulation coincides with the initial one.

In the whole process there are two sequences of reflections on the left from bottom to top (one for a $b$-chain and the other for a $w$-chain) and two similar sequences of reflections on the right from top to bottom, which is the action described in the lemma.

Corollary 5.10. $\mathrm{Za}^{h+n+1}=\mathrm{Id}$.

Proof. Let $R$ be the isomorphism from $\operatorname{Conf}_{q}^{p}(\mathcal{B})$ to $\operatorname{Conf}_{q p^{\circ}}^{e}(\mathcal{B})$ by reflections on the right. Elements in $\operatorname{Conf}_{q p^{\circ}}^{e}(\mathcal{B})$ are described as

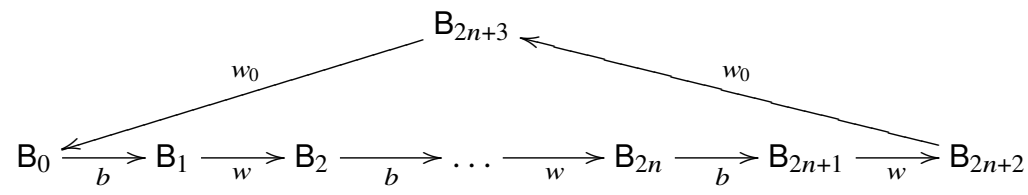

Consider the Coxeter element $c=b w$. We have $c^{h}=w_{0}^{2}$ as positive braids. Therefore, the chain $\mathrm{B}_{2 n+2} \stackrel{w_{0}}{\longrightarrow} \mathrm{B}_{2 n+3} \stackrel{w_{0}}{\longrightarrow} \mathrm{B}_{0}$ can be written as $h$ copies of $c$ chains as below, where $\mathrm{B}_{(i)}=\mathrm{B}_{2 i}$ for $0 \leq i \leq n+1$ :

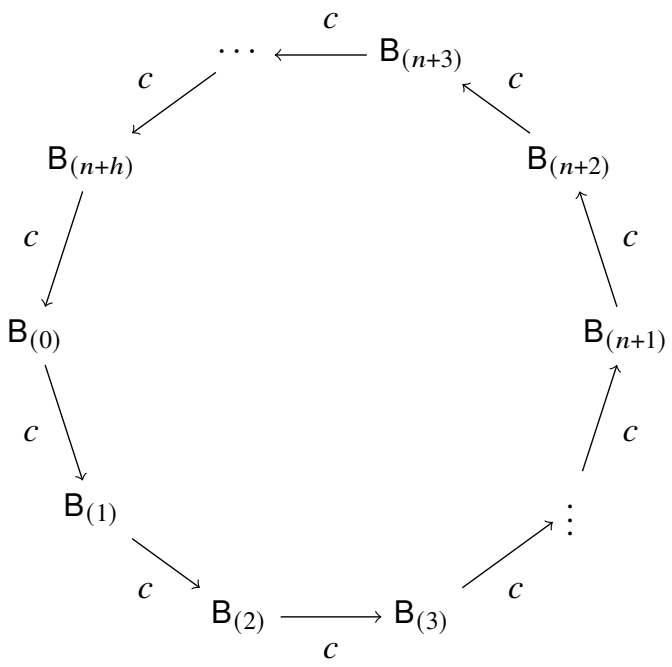

After conjugation by $R$, the transformation Za acts on $\operatorname{Conf}_{q p^{\circ}}^{e}(\mathcal{B})$ by rotating the above circle clockwise by one step. Therefore, $\mathrm{Za}^{h+n+1}=\mathrm{Id}$.

As in [Kel11, Sec.5.7], Zamolodchikov's periodicity implies that

$$
\left(\mathrm{DT}_{\Delta \square \Delta^{\prime}}\right)^{m}=\mathrm{Id}, \quad \text { where } m=\frac{2\left(h+h^{\prime}\right)}{\operatorname{gcd}\left(h, h^{\prime}\right)} .
$$

We close this section by presenting a direct proof of the above identity for $\Delta \square \mathrm{A}_{n}$.

Corollary 5.11. $\left(\mathrm{DT}_{\Delta \square \mathrm{A}_{n}}\right)^{m}=\mathrm{Id}$ where $m=\frac{2(h+n+1)}{\operatorname{gcd}(h, n+1)}$. 
Proof. In the braid group we have

$$
\left(q p^{\circ}\right)^{\frac{h}{\operatorname{gcd}(h, n+1)}}=(\underbrace{b w b w b w \ldots b w}_{n+1 \text { copies of } b w})^{\frac{h}{\operatorname{gcd}(h, n+1)}}=c^{\frac{h(n+1)}{\operatorname{gcd}(h, n+1)}}=\Omega^{\frac{(n+1)}{\operatorname{gcd}(h, n+1)}} .
$$

By Theorem 5.1, the order of the DT transformation of $\operatorname{Conf}_{q}^{p}(\mathcal{B})$ divides $\frac{2(h+n+1)}{\operatorname{gcd}(h, n+1)}$.

\section{Points Counting and Positive Braid Closures}

\subsection{Points Counting over a Finite Field}

The proof of Theorem 2.30 showed that the space $\operatorname{Conf}_{d}^{b}(\mathcal{A})$ can be realised as the nonvanishing locus of an integral polynomial given by Theorem 2.7. Therefore, $\operatorname{Conf}_{d}^{b}(\mathcal{A})$ is well defined over any finite field $\mathbb{F}_{q}$. In this section we present an algorithm ${ }^{7}$ counting its $\mathbb{F}_{q}$ points

$$
f_{d}^{b}(q):=\left|\operatorname{Conf}_{d}^{b}(\mathcal{A})\left(\mathbb{F}_{q}\right)\right|
$$

Let $u$ and $v$ be Weyl group elements and $s_{i}$ a simple reflection. We set

$$
P_{i}^{u, v}= \begin{cases}q & \text { if } v=s_{i} u \text { and } l\left(s_{i} u\right)<l(u) \\ q-1 & \text { if } v=u \text { and } l\left(s_{i} u\right)>l(u) \\ 1 & \text { if } v=s_{i} u \text { and } l\left(s_{i} u\right)>l(u) \\ 0 & \text { otherwise. }\end{cases}
$$

Theorem 6.2. Let $\mathbf{i}=\left(i_{1}, \ldots, i_{n}\right)$ be a word of $d b^{\circ}$. Then

$$
f_{d}^{b}(q)=(q-1)^{\tilde{r}} \sum_{\left(u_{1}, \ldots, u_{n-1}\right) \in \mathrm{W}^{n-1}} \prod_{k=1}^{n} P_{i_{k}}^{u_{k-1}, u_{k}},
$$

where $u_{0}=u_{n}=e$ and $\tilde{r}=\operatorname{dim} \mathrm{T}$.

Proof. Because $\operatorname{Conf}_{d}^{b}(\mathcal{A}) \stackrel{\sim}{=} \operatorname{Conf}_{d b^{\circ}}^{e}(\mathcal{A})$, it suffices to count $\mathbb{F}_{q}$-points of the latter space. Note that every configuration in $\operatorname{Conf}_{d b^{\circ}}^{e}(\mathcal{A})$ has a unique representative such that the pair of flags associated to its left side is $U_{+} \longrightarrow B_{-}$. Then let us fix a triangulation by taking diagonals from the top vertex to all of the bottom vertices and label all of the diagonals by Weyl group elements indicating the Tits codistances between the top flag and the bottom flags. It gives rise to a decomposition of the space $\operatorname{Conf}_{d b^{\circ}}^{e}(\mathcal{A})$. Moving across the triangulation from left to right, we associate the number of possible configurations to each triangle based on Lemma A.8: $q$ for $\mathbb{A}^{1}, q-1$ for $\mathbb{G}_{m}, 1$ for $\{*\}$ and 0 for $\emptyset$. In the end there is another decoration $\mathrm{A}_{n}$ over the last flag $\mathrm{B}_{n}$, which gives another multiple of $(q-1)^{\tilde{r}}=\left|\mathrm{T}\left(\mathbb{F}_{q}\right)\right|$. Taking the summation over all possible cases, we get (6.3).

Inspired by a recent result of Galashin and Lam [GL20] on computing the $\mathbb{F}_{q}$-point count of positroid cells, we relate the computation of $f_{d}^{b}(q)$ with Hecke algebras.

Let us first briefly recall the definition of Hecke algebra $\mathcal{H}$ associated with a generalised Cartan matrix $\mathrm{C}$. As a noncommutative algebra, $\mathcal{H}$ is generated over $\mathbb{Z}\left[q^{ \pm 1}\right]$ by the elements $\left\{T_{i}\right\}_{i \in \mathrm{S}}$, where $\mathrm{S}$ is the set of Coxeter generators. The generators $T_{i}$ satisfy the usual braid relations (A.4) imposed by the Cartan matrix as well as the following identity:

$$
\left(T_{i}+q\right)\left(T_{i}-1\right)=0 \quad \forall i \in \mathrm{S} .
$$

${ }^{7}$ This algorithm was suggested to us by J.H. Lu. 
Note that (6.4) implies that

$$
T_{i}^{-1}=q^{-1} T_{i}+\left(1-q^{-1}\right) \quad \forall i \in \mathrm{S} .
$$

For any positive braid $b \in \mathrm{Br}^{+}$with braid word $\left(i_{1}, i_{2}, \ldots, i_{l}\right)$, we define

$$
T_{b}:=T_{i_{1}} T_{i_{2}} \cdots T_{i_{l}}
$$

Note that this is well defined because the generators $T_{i}$ also satisfy the braid relations. Because any Weyl group element $w \in \mathrm{W}$ defines a unique positive braid via any reduced word of $w$, we define $T_{w}$ to be the corresponding product of the generators $T_{i}$ according to the reduced word. In particular, as a consequence of (6.4), for any Weyl group elements $u, v \in \mathrm{W}$ with $v=s_{i} u$, we have

$$
T_{i} T_{u}= \begin{cases}T_{v} & \text { if } l(v)>l(u), \\ (1-q) T_{u}+q T_{v} & \text { if } l(v)<l(u) .\end{cases}
$$

It is known that $\left\{T_{w}\right\}_{w \in \mathrm{W}}$ forms a linear basis of $\mathcal{H}$; that is, $\mathcal{H} \cong \bigoplus_{w \in \mathrm{W}} \mathbb{Z}\left[q^{ \pm 1}\right] T_{w}$. We define a $\mathbb{Z}\left[q^{ \pm 1}\right]$-linear map $\epsilon: \mathcal{H} \rightarrow \mathbb{Z}\left[q^{ \pm 1}\right]$ by

$$
\epsilon\left(T_{w}\right)=\left\{\begin{array}{l}
1 \text { if } w=e \\
0 \text { otherwise }
\end{array}\right.
$$

Corollary 6.7. Let $\mathbf{i}=\left(i_{1}, \ldots, i_{n}\right)$ be a word of $d b^{\circ}$ and let $\tilde{r}:=\operatorname{dim} \mathrm{T}$. Then

$$
f_{d}^{b}(q)=(q-1)^{\tilde{r}} q^{n} \epsilon\left(T_{d b^{\circ}}^{-1}\right)
$$

Proof. By comparison with (6.3), it suffices to show that

$$
q^{n} \epsilon\left(T_{d b^{\circ}}^{-1}\right)=\sum_{\left(u_{1}, \ldots, u_{n-1}\right) \in \mathrm{W}^{n-1}} \prod_{k=1}^{n} P_{i_{k}}^{u_{k-1}, u_{k}} .
$$

Without loss of generality, let us assume that $b=e$. Let $\left(i_{1}, i_{2}, \ldots, i_{n}\right)$ be a braid word for $d$. Then $T_{d}^{-1}=T_{i_{n}}^{-1} T_{i_{n-1}}^{-1} \cdots T_{1}^{-1}$. By substituting in (6.5), we get

$$
q^{n}\left(T_{d}^{-1}\right)=\left(T_{i_{n}}+(q-1)\right)\left(T_{i_{n-1}}+(q-1)\right) \cdots\left(T_{i_{1}}+(q-1)\right) .
$$

For each $0 \leq k \leq n$, define $h_{w}^{k}(q)$ to be the coefficients in the expansion

$$
\left(T_{i_{k}}+(q-1)\right) \cdots\left(T_{i_{1}}+(q-1)\right)=\sum_{w \in \mathrm{W}} h_{w}^{k}(q) T_{w} .
$$

We claim that for any $u_{k} \in \mathrm{W}$,

$$
\sum_{\left(u_{1}, \ldots, u_{k-1}\right) \in \mathrm{W}^{k-1}} \prod_{l=1}^{k} P_{i_{l}}^{u_{l}, u_{l-1}}=h_{u_{k}}^{k}(q),
$$

and the theorem will follow from this claim and the definition of the linear map $\epsilon$.

We will do an induction on $k$. The claim is trivial for the base case $k=0$. For the inductive step, we define $u:=u_{k}$ and $v:=s_{i_{k+1}} u$. If $l(v)>l(u)$, we have

$$
\left(T_{i_{k+1}}+(q-1)\right) h_{u}^{k}(q) T_{u}=h_{u}^{k}(q) T_{v}+(q-1) h_{u}^{k}(q) T_{u},
$$


which covers the middle two cases in (6.1). On the other hand, if $l(v)<l(u)$, then by (6.6) we have

$$
\left(T_{i_{k+1}}+(q-1)\right) h_{u}^{k}(q) T_{u}=h_{u}^{k}(q)\left((1-q) T_{u}+q T_{v}+(q-1) T_{u}\right)=h_{u}^{k}(q) q T_{v},
$$

which covers the first case in (6.1). By combining these cases, we see that (6.8) remains true for $k+1$ and hence the induction is finished.

The group $\mathrm{T} \times \mathrm{T}$ acts on $\mathrm{Conf}_{d}^{b}(\mathcal{A})$ by altering the decorations $\mathrm{A}^{0}$ and $\mathrm{A}_{n}$. It induces a transitive $\mathrm{T} \times \mathrm{T}$ action on each fibre of the projection $\pi: \operatorname{Conf}_{d}^{b}(\mathcal{A}) \rightarrow \operatorname{Conf}_{d}^{b}(\mathcal{B})$. As stacks we have the isomorphism

$$
\operatorname{Conf}_{d}^{b}(\mathcal{A}) /(\mathrm{T} \times \mathrm{T}) \stackrel{\sim}{=} \operatorname{Conf}_{d}^{b}(\mathcal{B}) .
$$

Therefore, the number of $\mathbb{F}_{q}$-points of $\operatorname{Conf}_{d}^{b}(\mathcal{B})$ as a stack is

$$
g_{d}^{b}(q):=\left|\operatorname{Conf}_{d}^{b}(\mathcal{B})\left(\mathbb{F}_{q}\right)\right|=\frac{\left|\operatorname{Conf}_{d}^{b}(\mathcal{A})\left(\mathbb{F}_{q}\right)\right|}{\left|\mathrm{T} \times \mathrm{T}\left(\mathbb{F}_{q}\right)\right|}=\frac{f_{d}^{b}(q)}{(q-1)^{2 \tilde{r}}}
$$

Note that, in general, $g_{d}^{b}(q)$ is a rational function with possible poles at $q=1$. We include the code of a Python program that computes $g_{d}^{b}(q)$ for positive braid closures in Appendix A.3.

Example 6.9. Let $\mathrm{G}=\mathrm{SL}_{2}, b=e$ and $d=s_{1} s_{1} s_{1}$. Let us fix a triangulation given by drawing diagonals from the top vertex to all of the vertices at the bottom. Below are all possible cases of different Tits codistances:
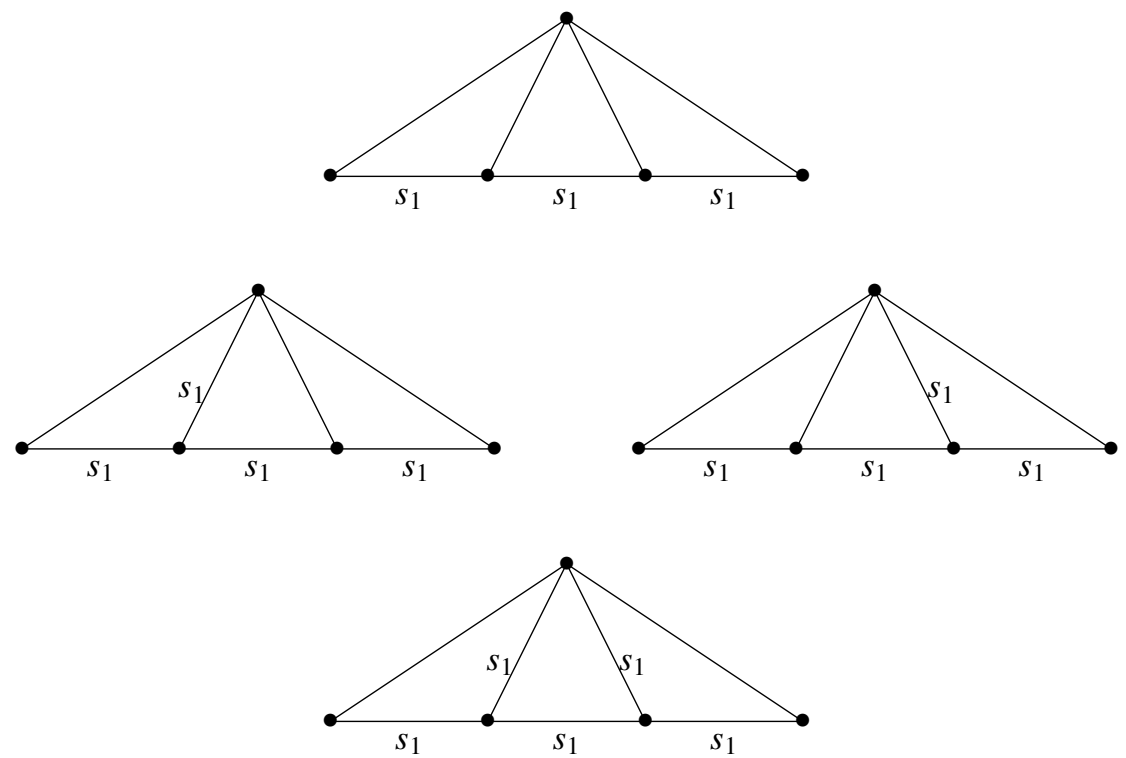

The first case gives $(q-1)^{3}$, the fourth case gives 0 and each of the rest give $q(q-1)$. Therefore,

$$
\begin{aligned}
& f_{d}^{b}(q)=\left((q-1)^{3}+2 q(q-1)\right)(q-1)=q^{4}-2 q^{3}+2 q^{2}-2 q+1 \\
& g_{d}^{b}(q)=\frac{f_{d}^{b}(q)}{(q-1)^{2}}=q^{2}+1
\end{aligned}
$$

Remark 6.10. This example coincides with Example 6.38 in [STZ17]. In the next section, we show that $\operatorname{Conf}_{d}^{b}(\mathcal{B})$ is isomorphic to the moduli space $\mathcal{M}_{1}\left(\Lambda_{d}^{b}\right)$ of microlocal rank-1 sheaves in [STZ17]. 


\subsection{Legendrian links and Microlocal Rank-1 Sheaves}

Let us briefly recall some basic definitions about Legendrian links. The space $\mathbb{R}^{3}$ is equipped with the standard contact structure from the 1 -form $\alpha=y \mathrm{~d} x-\mathrm{d} z$. A Legendrian link in $\mathbb{R}^{3}$ is a link $\Lambda$ such that the restriction of $\alpha$ to $\Lambda$ vanishes. Two links are Legendrian isotopic if there is an isotopy between them that preserves the property of being Lengendrian at every stage. A Legendrian link $\Lambda$ can be visualised by its image under the front projection $\pi_{F}$ from $\mathbb{R}^{3}$ to the $x z$-plane. The constraint $\left.\alpha\right|_{\Lambda}=0$ implies that the $y$ coordinate of $\Lambda$ is determined by the slope of its front projection.

Shende, Treumann and Zaslow [STZ17] have associated to every Legendrian link $\Lambda$ a category $\mathbf{S h}_{\Lambda}^{\bullet}\left(\mathbb{R}^{2}\right)$ of constructible sheaves on the $x z$ plane with singular support controlled by the front projection of $\Lambda$. Using a theorem of Guillermou-Kashiwara-Schapira [GKS12], they proved that the category $\mathbf{S h}_{\Lambda}^{\bullet}\left(\mathbb{R}^{2}\right)$ is invariant under Legendrian isotopies. As a consequence, the moduli space $\mathcal{M}_{1}(\Lambda)$ of microlocal rank-1 sheaves in $\mathbf{S h}_{\Lambda}^{\bullet}\left(\mathbb{R}^{2}\right)$ is a Legendrian link invariant.

In this section we investigate Legendrian links arising from a pair $(b, d)$ of positive braids of Dynkin type $\mathrm{A}_{r}$. Let $\mathbf{i}$ and $\mathbf{j}$ be reduced words of $b$ and $d$, respectively. Associated to $(\mathbf{i}, \mathbf{j})$ is a Legendrian link $\Lambda_{\mathbf{j}}^{\mathbf{i}}$, whose front projection is described by the following steps:

(1) We draw $2 r+2$ many horizontal strands on the $x z$-plane.

(2) The top $r+1$ strands have crossings encoded by $\mathbf{i}$ and the bottom $r+1$ strands have crossings encoded by $\mathbf{j}$.

(3) We close up both ends of the strands by cusps.

Example 6.11. Let $r=2$. Let $\mathbf{i}=(1,2)$ and $\mathbf{j}=(1)$. The front projection $\pi_{F}\left(\Lambda_{\mathbf{j}}^{\mathbf{i}}\right)$ of $\Lambda_{\mathbf{j}}^{\mathbf{i}}$ is

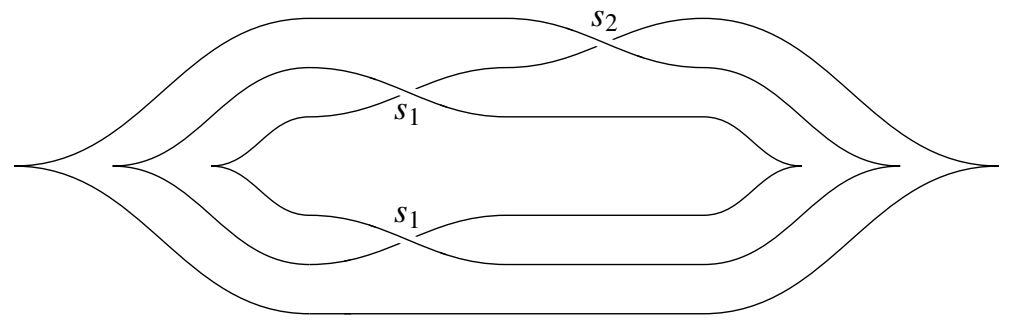

Connected components of the complement of $\pi_{F}\left(\Lambda_{\mathbf{j}}^{\mathbf{i}}\right)$ are called faces. We use $f_{\text {in }}$ to denote the face enclosed by the $(r+1)$-th and the $(r+2)$-th strands and $f_{\text {out }}$ to denote the unbounded face. Crossings and cusps of $\pi_{F}\left(\Lambda_{\mathbf{j}}^{\mathbf{i}}\right)$ cut its strands into segments called edges. Two faces are said to be neighbouring if they are separated by an edge $e$. Note that one of the neighbouring faces is above $e$ and the other is below $e$ with respect to the $z$-direction in the $x z$ plane.

Let us present an equivalent working definition of microlocal rank-1 sheaves associated to $\Lambda_{\mathbf{j}}^{\mathbf{i}}$. See [STZ17] for the original definition.

Definition 6.12. A microlocal rank-1 sheaf $\mathcal{F}$ associated to $\Lambda_{\mathbf{j}}^{\mathbf{i}}$ consists of the following data:

$\circ$ assigned to every face $f$ is a finite-dimensional vector space $V_{f}$ over a field $\mathrm{k}$;

$\circ$ assigned to every edge $e$ is a full-rank linear map $\phi_{e}: V_{f} \rightarrow V_{g}$, where $f$ and $g$ are neighbouring

faces separated by $e$, with $f$ sitting below $e$

such that

$\circ$ the dimensions of vector spaces assigned to any neighbouring faces differ by 1 ;

$\circ \operatorname{dim} V_{f_{\text {in }}}=r+1$ and $\operatorname{dim} V_{f_{\text {out }}}=0$;

$\circ$ for every crossing illustrated on the left below, the following sequence is exact:

$$
V_{s} \stackrel{\left(\phi_{s w}, \phi_{s e}\right)}{\longrightarrow} V_{w} \oplus V_{e} \stackrel{\phi_{w n}-\phi_{e n}}{\longrightarrow} V_{n}
$$


$\circ$ for every cusp illustrated on the right below, $\phi_{f g} \circ \phi_{g f}=\mathrm{id}_{V_{f}}$ :
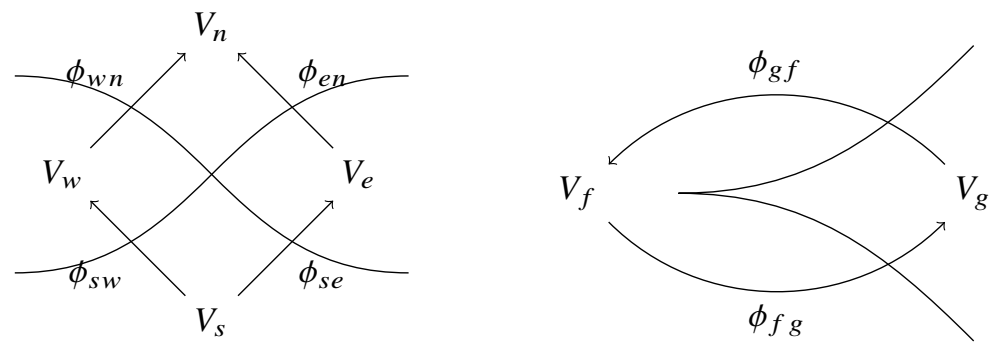

One can think of a microlocal rank-1 sheaf as a quiver representation and two microlocal rank-1 sheaves are isomorphic if they are isomorphic as quiver representations. Let $\mathcal{M}_{1}\left(\Lambda_{\mathbf{j}}^{\mathbf{i}}\right)$ be the moduli space of isomorphism classes of microlocal rank-1 sheaves associated to $\Lambda_{\mathbf{j}}^{\mathbf{i}}$. The space $\mathcal{M}_{1}\left(\Lambda_{\mathbf{j}}^{\mathbf{i}}\right)$ is invariant under Legendrian isotopies.

Theorem 6.14. Let $\mathbf{i}$ and $\mathbf{j}$ be reduced words of positive braids $b$ and $d$, respectively. There exists $a$ natural isomorphism from $\mathcal{M}_{1}\left(\Lambda_{\mathbf{j}}^{\mathbf{i}}\right)$ to $\operatorname{Conf}_{d}^{b}(\mathcal{B})$.

Proof. Let $\mathcal{F}$ be a a microlocal rank-1 sheaf. Let us align the cusps of $\pi_{F}\left(\Lambda_{\mathbf{j}}^{\mathbf{i}}\right)$ on a horizontal line that separates the $x z$-plane into two halves. We slice up the bottom half vertically at the crossings. For each slice, the sheave $\mathcal{F}$ gives rise to $r+2$ vector spaces with full-ranked linear maps connecting them. See the first graph in Figure 1. Let

$$
U_{i}:=\operatorname{Im}\left(V_{i} \rightarrow \ldots \rightarrow V_{r+1}\right)
$$

By Definition 6.12, every local linear map is a codimension 1 inclusion. Therefore, we obtain a complete flag B $=\left(0=U_{0} \subset U_{1} \subset \cdots \subset U_{r+1}=V_{f_{\text {in }}}\right)$.

Let $\mathrm{B}=\left(U_{0} \subset U_{1} \subset \cdots \subset U_{r} \subset U_{r+1}\right)$ and $\mathrm{B}^{\prime}=\left(U_{0}^{\prime} \subset U_{1}^{\prime} \subset \cdots \subset U_{r}^{\prime} \subset U_{r+1}^{\prime}\right)$ be flags associated to two adjacent slices sharing a crossing on the $j$ th level. By the exactness condition (6.13), we get $U_{j} \neq U_{j}^{\prime}$ and $U_{i}=U_{i}^{\prime}$ if $i \neq j$. Therefore, $\mathrm{B} \stackrel{s_{j}}{\longrightarrow} \mathrm{B}^{\prime}$ in $\mathcal{B}_{-}$. Because the crossings of the strands in the bottom half are encoded by $\mathbf{j}$, by associating flags to the slices, we get a $\mathbf{j}$-chain

$$
\mathrm{B}_{0} \stackrel{s_{j_{1}}}{\longrightarrow} \mathrm{B}_{1} \stackrel{s_{j_{2}}}{\longrightarrow} \ldots \stackrel{s_{j_{n}}}{\longrightarrow} \mathrm{B}_{n}
$$

Similarly, let us slice up the top half vertically at the crossings. For each slice, we get the data as shown on the the second graph in Figure 1. The dimension condition forces $\operatorname{dim} V_{i}=i$ and the linear maps are all surjective. Let

$$
W_{i}:=\operatorname{ker}\left(V_{r+1} \rightarrow V_{r} \rightarrow \cdots \rightarrow V_{i}\right)
$$

It determines a complete flag B $=\left(0=W_{r+1} \subset W_{r} \subset \ldots W_{0}=V_{f_{\text {in }}}\right)$. Similarly, a crossing on the $i$ th level at the top half imposes the condition that two adjacent flags are of Tits codistance $s_{i}$ in the flag variety $\mathcal{B}_{+}$. Therefore, the top half of a microlocal rank-1 sheaf $\mathcal{F}$ gives rise to an $\mathbf{i}$-chain

$$
\mathrm{B}^{0} \stackrel{s_{i_{1}}}{\longrightarrow} \mathrm{B}^{1} \stackrel{s_{i_{2}}}{\longrightarrow} \ldots \stackrel{s_{i_{m}}}{\longrightarrow} \mathrm{B}^{m}
$$

Now we have obtained an $\mathbf{i}$-chain and a $\mathbf{j}$-chain from $\mathcal{F}$. From the construction of the braid closure, we know that the left cusps are nested as in the third graph of Figure 1. By Definition 6.12, the composition 

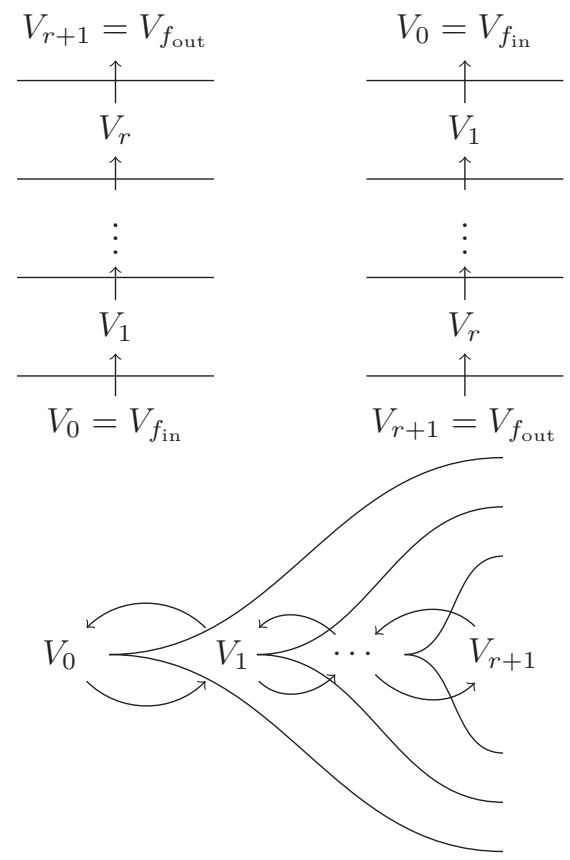

Figure 1. Flags obtained from microlocal rank-1 sheaves.

$V_{i} \rightarrow V_{r+1} \rightarrow V_{i}$ is the identity map $\operatorname{id}_{V_{i}}$. Therefore,

$$
U_{i} \cap W_{i}:=\operatorname{im}\left(V_{i} \rightarrow V_{r+1}\right) \cap \operatorname{ker}\left(V_{r+1} \rightarrow V_{i}\right)=0 .
$$

Therefore, the flags $\mathrm{B}^{0}$ and $\mathrm{B}_{0}$ are in general position. Similarly, the flags $\mathrm{B}^{m}$ and $\mathrm{B}_{n}$ are in general position. Putting them together, we get a configuration

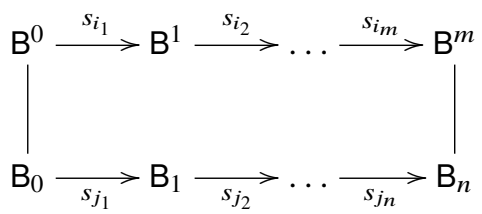

This defines a map

$$
\mathcal{M}_{1}\left(\Lambda_{\mathbf{j}}^{\mathbf{i}}\right) \rightarrow \operatorname{Conf}_{d}^{b}(\mathcal{B})
$$

It is not hard to show that this construction can be reversed to get an isomorphism class of microlocal rank-1 sheaves from a point in $\operatorname{Conf}_{d}^{b}(\mathcal{B})$.

Theorem 6.14 is a slight generalisation of [STZ17, Prop 1.5]. It shows that $\mathcal{M}_{1}\left(\Lambda_{\mathbf{j}}^{\mathbf{i}}\right)$ is equipped with a natural cluster Poisson structure. As a direct consequence, we obtain the following.

Corollary 6.15. The space $\operatorname{Conf}_{d}^{b}(\mathcal{B})$ (as an algebraic stack) and $g_{d}^{b}(q)$ are Legendrian link invariants for closures of positive braids $(b, d)$. 


\section{A. Appendix}

\section{A.1. Basics about Kac-Peterson Groups}

In this appendix we collect the necessary information on Kac-Peterson groups (a.k.a. minimal KacMoody groups). We will mostly follow S. Kumar [Kum02].

A generalised Cartan matrix is a matrix $\mathrm{C}=\left(\mathrm{C}_{i j}\right)$ whose diagonal entries are all 2 and whose off-diagonal entries are nonpositive integers, such that $\mathrm{C}_{i j}=0$ if and only if $\mathrm{C}_{j i}=0$.

A realisation of an $r \times r$ generalised Cartan matrix $C$ is a quadruple $\left(\mathfrak{h}, \mathfrak{h}^{*}, \Pi^{\vee}, \Pi\right)$ such that

$\circ \mathfrak{h}$ and $\mathfrak{h}^{*}$ are dual complex vector spaces of dimension $\tilde{r}:=r+l$, where $l$ is the corank of $\mathrm{C}$;

$\circ \Pi=\left\{\alpha_{1}, \ldots, \alpha_{r}\right\} \subset \mathfrak{h}^{*}$ and $\Pi^{\vee}=\left\{\alpha_{1}^{\vee}, \ldots, \alpha_{r}^{\vee}\right\} \subset \mathfrak{h}$ are linearly independent subsets;

$\circ\left\langle\alpha_{i}^{\vee}, \alpha_{j}\right\rangle=\mathrm{C}_{i j}$ for $i, j=1, \ldots, r$.

Every C admits a unique up to isomorphism realisation ([Kac83, Prop. 1.1]).

The Kac-Moody algebra $\mathfrak{g}_{\mathrm{C}}$ associated to $\mathrm{C}$ is a Lie algebra, with the generators $E_{i}, E_{-i}(i=1, \ldots, r)$ and $\mathfrak{h}$ and the relations

$$
\begin{cases}{\left[H, H^{\prime}\right]=0} & \left(H, H^{\prime} \in \mathfrak{h}\right), \\ {\left[H, E_{i}\right]=\left\langle H, \alpha_{i}\right\rangle E_{i},} & \\ {\left[H, E_{-i}\right]=-\left\langle H, \alpha_{i}\right\rangle E_{i}} & (i=1, \ldots r ; H \in \mathfrak{h}), \\ {\left[E_{i}, E_{-j}\right]=\delta_{i j} \alpha_{i}^{\vee}} & (i, j=1, \ldots, r), \\ \operatorname{ad}_{E_{i}}^{1-C_{i j}} E_{j}=0, & \\ \operatorname{ad}_{E_{-i}}^{1-C_{i j}} E_{-j}=0 & (i \neq j) .\end{cases}
$$

From now on, let $\mathrm{C}$ be a symmetrisable generalised Cartan matrix; that is, there is an invertible diagonal matrix $D$ such that $D^{-1} C$ is symmetric. The matrix $D$ may be chosen such that its diagonal entries are positive integers with gcd $=1$. Let $\left(\mathfrak{h}, \mathfrak{h}^{*}, \Pi^{\vee}, \Pi\right)$ be a realisation of $C$. We further fix once and for all a lattice $P \subset \mathfrak{h}^{*}$ with a basis $\left\{\omega_{1}, \ldots, \omega_{\tilde{r}}\right\}$ such that $\Pi \subset P$ and

$$
\left\langle\alpha_{i}^{\vee}, \omega_{j}\right\rangle=\delta_{i j} \quad \text { for } i=1, \ldots, r \text { and } j=1, \ldots, \tilde{r} .
$$

The lattice $\mathrm{P}$ is called the weight lattice. A weight $\lambda \in \mathrm{P}$ is dominant if $\left\langle\alpha_{i}^{\vee}, \lambda\right\rangle \geq 0$ for every $\alpha_{i}^{\vee} \in \Pi$. Denote by $\mathrm{P}_{+}$the set of dominant weights.

The elements $\omega_{1}, \ldots, \omega_{\tilde{r}}$ are called the fundamental weights. They extend $\mathrm{C}$ to an $\tilde{r} \times \tilde{r}$ matrix

$$
\tilde{\mathrm{C}}=\left(\mathrm{C}_{i j}\right)=\left(\begin{array}{cc}
\mathrm{C} & \mathrm{DA} \\
\mathrm{A} & 0
\end{array}\right)
$$

such that $\alpha_{j}=\sum_{i=1}^{\tilde{r}} \mathrm{C}_{i j} \omega_{i}$ for $j=1, \ldots, r$.

Lemma A.2. The matrix $\tilde{\mathrm{C}}$ is invertible.

Proof. The matrix C is of corank $l$. We may apply elementary column transformations to the first $r$ columns of $\tilde{C}$ and obtain a matrix

$$
\tilde{C^{\prime}}=\left(\begin{array}{ccc}
0 & C^{\prime} & D A \\
L & * & 0
\end{array}\right) .
$$

The first $r$ column vectors of $\tilde{C}$ are linearly independent because $\Pi$ is a linearly independent subset of $\mathfrak{h}^{*}$. Therefore, the $l \times l$ submatrix $\mathrm{L}$ is invertible. Meanwhile, the matrix $\tilde{\mathrm{C}}$ is symmetrisable. Therefore, the first $r$ row vectors of $\tilde{C}$ are linearly independent and the $r \times r$ submatrix ( $\left.C^{\prime} \mathrm{DA}\right)$ of $\tilde{\mathrm{C}}^{\prime}$ is invertible. It follows then that $\tilde{C^{\prime}}$ (and hence $\tilde{C}$ ) is invertible. 
Using the matrix $\tilde{\mathrm{C}}$ we can extend $\Pi$ to a basis $\left\{\alpha_{i}\right\}_{i=1}^{\tilde{r}}$ of $\mathfrak{h}^{*}$ such that

$$
\alpha_{j}=\sum_{i=1}^{\tilde{r}} \mathrm{C}_{i j} \omega_{i}
$$

Let $\left\{\alpha_{i}^{\vee}\right\}_{i=1}^{\tilde{r}}$ and $\left\{\omega_{i}^{\vee}\right\}_{i=1}^{\tilde{r}}$ be, respectively, the dual basis of $\left\{\omega_{i}\right\}_{i=1}^{\tilde{r}}$ and $\left\{\alpha_{i}\right\}_{i=1}^{\tilde{r}}$. Then

$$
\alpha_{i}^{\vee}=\sum_{j=1}^{\tilde{r}} \mathrm{C}_{i j} \omega_{j}^{\vee} \quad \text { and } \quad\left\langle\alpha_{i}^{\vee}, \alpha_{j}\right\rangle=\mathrm{C}_{i j} \quad(i, j=1, \ldots, \tilde{r}) \text {. }
$$

Define $\mathrm{Q}:=\bigoplus_{i=1}^{\tilde{r}} \mathbb{Z} \alpha_{i} \subset \mathrm{P}$. The quotient group $\mathrm{P} / \mathrm{Q}$ is a finite abelian group of $\operatorname{order}|\operatorname{det}(\tilde{\mathrm{C}})|$.

We define two algebraic tori

$$
\mathrm{T}_{\mathrm{sc}}:=\operatorname{Hom}\left(\mathrm{P}, \mathbb{G}_{m}\right) \quad \text { and } \quad \mathrm{T}_{\mathrm{ad}}:=\operatorname{Hom}\left(\mathrm{Q}, \mathbb{G}_{m}\right)
$$

Both $T_{\text {sc }}$ and $T_{\text {ad }}$ have $\mathfrak{h}$ as their Lie algebras. The embedding $Q \subset P$ induces a surjective homomorphism from $T_{s c}$ to $T_{a d}$, whose kernel $Z$ is isomorphic to $P / Q$.

The Kac-Peterson group $\mathrm{G}_{\mathrm{sc}}$ (respectively $\mathrm{G}_{\mathrm{ad}}$ ) is generated by $\mathrm{T}_{\mathrm{sc}}$ (respectively $\mathrm{T}_{\mathrm{ad}}$ ) and the oneparameter groups

$$
\bigcup_{i}:=\left\{\exp \left(p E_{i}\right) \mid p \in \mathbb{G}_{a}\right\}, \quad i \in\{ \pm 1, \ldots, \pm r\},
$$

with relations determined by (A.1). They are also known as the minimal Kac-Moody groups, in the sense that they are constructed by only exponentiating the real root spaces of $\mathfrak{g}_{\mathrm{C}}$. The group $\mathrm{Z}$ is contained in the centre of $G_{s c}$. The surjection from $T_{s c}$ to $T_{a d}$ induces a $|Z|$-to-1 covering map

$$
\pi: \mathrm{G}_{\mathrm{sc}} \longrightarrow \mathrm{G}_{\mathrm{ad}}
$$

We refer the reader to [Kum02, Section 7.4] for more details on Kac-Peterson groups.

Notation A.3. We will write $e_{i}(p)$ instead of $\exp \left(p E_{i}\right)$ and omit the argument $p$ if $p=1$. Let T be either $\mathrm{T}_{\mathrm{sc}}$ or $\mathrm{T}_{\mathrm{ad}}$. For a character $\lambda$ of $\mathrm{T}$ and $t \in \mathrm{T}$, we set $t^{\lambda}:=\lambda(t)$. For a cocharacter $\lambda^{\vee}$ of $\mathrm{T}$ and $p \in \mathbb{G}_{m}$, we set $p^{\lambda^{\vee}}:=\lambda^{\vee}(p)$.

Let $\mathrm{G}$ be either $\mathrm{G}_{\mathrm{sc}}$ or $\mathrm{G}_{\mathrm{ad}}$. Let $\mathrm{N}$ be the normaliser of $\mathrm{T}$ in $\mathrm{G}$. The Weyl group $\mathrm{W}:=\mathrm{N} / \mathrm{T}$ is generated by $S:=\left\{s_{i}\right\}_{i=1}^{r}$ with the relations $s_{i}^{2}=1$ for all $i$ together with the braid relations

$$
\underbrace{s_{i} s_{j} \ldots}_{m_{i j}}=\underbrace{s_{j} s_{i} \ldots}_{m_{i j}} \quad \forall i \neq j
$$

where $m_{i j}=2,3,4,6$ or $\infty$ according to whether $C_{i j} C_{j i}$ is $0,1,2,3$ or $\geq 4$. The elements

$$
\bar{s}_{i}:=e_{i}^{-1} e_{-i} e_{i}^{-1} \quad \text { and } \quad \overline{\bar{s}}_{i}:=e_{i} e_{-i}^{-1} e_{i}
$$

are both coset representatives of $s_{i} \in \mathrm{N} / \mathrm{T}$. They satisfy the braid relations and therefore determine two natural representatives for every $w \in \mathrm{N} / \mathrm{T}$, which are denoted as $\bar{w}$ and $\overline{\bar{w}}$.

Let $\mathrm{U}_{+}$(respectively $\mathrm{U}_{-}$) be the subgroup of $\mathrm{G}$ generated by $\mathrm{U}_{1}, \ldots, \mathrm{U}_{r}$ (respectively $\mathrm{U}_{-1}, \ldots, \mathrm{U}_{-r}$ ). Define the Borel subgroups

$$
\mathrm{B}_{+}:=\mathrm{U}_{+} \mathrm{T}, \quad \mathrm{B}_{-}:=\mathrm{U}_{-} \mathrm{T} .
$$


The transposition $g \mapsto g^{t}$ is an involutive anti-automorphism of G such that

$$
e_{i}(p)^{t}:=e_{-i}(p) \quad \forall i \in \pm 1, \ldots, \pm r ; \quad h^{t}=h \quad \forall h \in \mathrm{T} .
$$

The transposition swaps $\mathrm{B}_{+}$and $\mathrm{B}_{-}$.

Recall the definition of Tits system in [Kum02, Section 5.1]. The tuple (G, $\left.\mathrm{B}_{+}, \mathrm{B}_{-}, \mathrm{N}, \mathrm{S}\right)$ forms a twin Tits system; that is,

$\circ$ the quadruples $\left(\mathrm{G}, \mathrm{B}_{+}, \mathrm{N}, \mathrm{S}\right)$ and $\left(\mathrm{G}, \mathrm{B}_{-}, \mathrm{N}, \mathrm{S}\right)$ are Tits systems;

$\circ$ if $l\left(w s_{i}\right)<l(w)$, then $\mathrm{B}_{-} w \mathrm{~B}_{+} s_{i} \mathrm{~B}_{+}=\mathrm{B}_{-} w s_{i} \mathrm{~B}_{+}$;

$\circ \mathrm{B}_{-} s_{i} \cap \mathrm{B}_{+}=\emptyset$ for $i=1, \ldots, r$.

From the twin Tits system we obtain two Borel decompositions and a Birkhoff decomposition

$$
\mathrm{G}=\bigsqcup_{u \in \mathrm{W}} \mathrm{B}_{+} u \mathrm{~B}_{+}=\bigsqcup_{v \in \mathrm{W}} \mathrm{B}_{-} v \mathrm{~B}_{-}, \quad \mathrm{G}=\bigsqcup_{w \in \mathrm{W}} \mathrm{B}_{-} w \mathrm{~B}_{+} .
$$

Recall the flag varieties $\mathcal{B}_{+}=\mathrm{G} / \mathrm{B}_{+}$and $\mathcal{B}_{-}=\mathrm{G} / \mathrm{B}_{-}$. As in Subsection 2.1, the above decompositions induce Tits distance functions $d_{ \pm}: \mathcal{B}_{ \pm} \times \mathcal{B}_{ \pm} \rightarrow \mathrm{W}$ and a Tits codistance function $d: \mathcal{B}_{+} \times \mathcal{B}_{-} \rightarrow \mathrm{W}$. The quintuple $\left(\mathrm{B}_{ \pm}, d_{ \pm}, d\right)$ is an example of twin buildings.

Proof of Lemma 2.5. For the first case, without loss of generality, let us assume that B, $\mathrm{B}^{\prime}, \mathrm{B}^{\prime \prime} \in \mathcal{B}_{+}$. From the assumption $u v=w$ and $l(u)+l(v)=l(w)$ we get

$$
\mathrm{B}_{+} w \mathrm{~B}_{+}=\mathrm{B}_{+} u \mathrm{~B}_{+} v \mathrm{~B}_{+} .
$$

Therefore, if $\mathrm{B} \stackrel{u}{\longrightarrow} \mathrm{B}^{\prime} \stackrel{v}{\longrightarrow} \mathrm{B}^{\prime \prime}$, then $\mathrm{B} \stackrel{w}{\longrightarrow} \mathrm{B}^{\prime \prime}$. Conversely, if $\mathrm{B} \stackrel{w}{\longrightarrow} \mathrm{B}^{\prime \prime}$, then there exists a flag $\mathrm{B}^{\prime}$ such that $\mathrm{B} \stackrel{u}{\longrightarrow} \mathrm{B}^{\prime} \stackrel{v}{\longrightarrow} \mathrm{B}^{\prime \prime}$. Itremains to show the uniqueness of $\mathrm{B}^{\prime}$.

Assume $v=s_{i}$. Let $\mathrm{B}^{\prime \prime \prime}$ satisfy $\mathrm{B} \stackrel{u}{\longrightarrow} \mathrm{B}^{\prime \prime \prime} \stackrel{s_{i}}{\longrightarrow} \mathrm{B}^{\prime \prime}$. Note that $\mathrm{B}_{+} s_{i} \mathrm{~B}_{+} s_{i} \mathrm{~B}_{+}=\mathrm{B}_{+} \sqcup \mathrm{B}_{+} s_{i} \mathrm{~B}_{+}$. If $\mathrm{B}^{\prime} \neq \mathrm{B}^{\prime \prime \prime}$, then we get $\mathrm{B}^{\prime} \stackrel{s_{i}}{\longrightarrow} \mathrm{B}^{\prime \prime \prime}$. Putting all of the flags together, we get

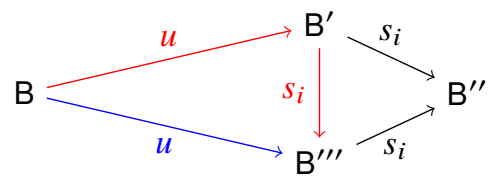

Because $l\left(u s_{i}\right)=l(u)+1$, from the red arrows we get $\mathrm{B} \stackrel{u s_{i}}{\longrightarrow} \mathrm{B}^{\prime \prime \prime}$, which contradicts with the blue arrow $\mathrm{B} \stackrel{u}{\longrightarrow} \mathrm{B}^{\prime \prime \prime}$. Therefore, $\mathrm{B}^{\prime}=\mathrm{B}^{\prime \prime \prime}$. For general $v$, we first fix a reduced word $v=s_{i_{1}} s_{i_{2}} \ldots s_{i_{l}}$. By the above discussion, there is a unique $\mathrm{B}^{\prime \prime \prime}$ such that $\mathrm{B} \stackrel{w s_{i_{l}}}{\longrightarrow} \mathrm{B}^{\prime \prime \prime} \stackrel{s_{i_{l}}}{\longrightarrow} \mathrm{B}^{\prime \prime}$. Then we focus on $\mathrm{B} \stackrel{w s_{i l}}{\longrightarrow} \mathrm{B}^{\prime \prime \prime}$. The uniqueness of $\mathrm{B}^{\prime}$ follows by induction on the length of $v$.

For the third case, using the second condition of twin Tits system recursively, we get

$$
\mathrm{B}_{-} u \mathrm{~B}_{+}=\mathrm{B}_{-} w \mathrm{~B}_{+} v^{-1} \mathrm{~B}_{+} .
$$

Therefore, if $\mathrm{B}_{0} \stackrel{w}{-} \mathrm{B}^{-1} \stackrel{v^{-1}}{\longrightarrow} \mathrm{B}^{0}$, then $\mathrm{B}_{0} \stackrel{u}{{ }^{0}} \mathrm{~B}^{0}$. Conversely, if $\mathrm{B}_{0} \stackrel{u}{\mathrm{~B}^{0}}$, then there exists

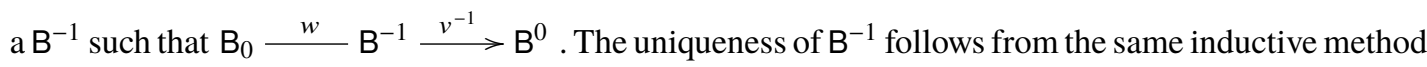
as in the proof of the first.

All other cases are analogous to the third case.

Next let us investigate the space of flags that are of Tits distance $s_{i}$ from a fixed flag. 
Lemma A.6. If $\mathrm{B}_{+} \stackrel{s_{i}}{\longrightarrow} \mathrm{B}$, then $\mathrm{B}=e_{i}(q) \bar{s}_{i} \mathrm{~B}_{+}$for some $q \in \mathbb{A}^{1}$.

Proof. The space of flags of Tits distance $s_{i}$ to $\mathrm{B}_{+}$is the quotient $\left(\mathrm{B}_{+} s_{i} \mathrm{~B}_{+}\right) / \mathrm{B}_{+}$. By Lemma 6.1.3 of [Kum02], we get $\mathrm{B}_{+}=\cup_{i} \mathrm{Q}_{i}$, where the subgroup $\mathrm{Q}_{i}=\mathrm{B}_{+} \cap s_{i} \mathrm{~B}_{+} s_{i}$. Therefore,

$$
\left(\mathrm{B}_{+} s_{i} \mathrm{~B}_{+}\right) / \mathrm{B}_{+}=\left(\bigcup_{i} \mathrm{Q}_{i} s_{i} \mathrm{~B}_{+}\right) / \mathrm{B}_{+}=\left(\bigcup_{i} s_{i} \mathrm{Q}_{i} \mathrm{~B}_{+}\right) / \mathrm{B}_{+}=\left(\bigcup_{i} s_{i} \mathrm{~B}_{+}\right) / \mathrm{B}_{+}=\left\{e_{i}(q) \bar{s}_{i} \mathrm{~B}_{+}\right\} .
$$

Corollary A.7. Let $\mathrm{B}$ be in either $\mathcal{B}_{+}$or $\mathcal{B}_{-}$. The space of flags of Tits distance $s_{i}$ away from $\mathrm{B}$ is isomorphic to $\mathbb{A}^{1}$.

Proposition A.8. Let $u, v \in \mathrm{W}$ and let $s_{i}$ be a simple reflection. Fix a pair $\mathrm{B}_{0}-u-\mathrm{B}^{0}$. Then the space of flags B that fits into either of the triangles
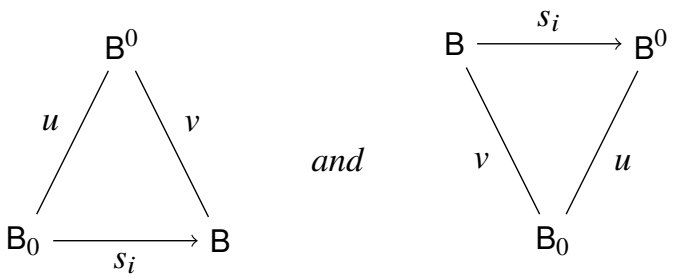

is isomorphic to

$$
\left\{\begin{array}{l}
\mathbb{A}^{1} \text { if } v=s_{i} u \text { and } l\left(s_{i} u\right)<l(u) \\
\mathbb{G}_{m} \text { if } v=u \text { and } l\left(s_{i} u\right)>l(u) \\
\{*\} \text { if } v=s_{i} u \text { and } l\left(s_{i} u\right)>l(u) \\
\emptyset \quad \text { otherwise. }
\end{array}\right.
$$

Proof. By symmetry, we will only prove the first case. Without loss of generality, let $\mathrm{B}^{0}=u \mathrm{~B}_{+}$and $\mathrm{B}_{0}=\mathrm{B}_{-}$. The set of flags of Tits distance $s_{i}$ to $\mathrm{B}_{-}$is

$$
\left\{\mathrm{B}_{-} e_{i}(p) \mid p \in \mathbb{G}_{m}\right\} \sqcup\left\{\mathrm{B}_{-} s_{i}\right\} .
$$

If $l\left(s_{i} u\right)<l(u)$, then we obtain the the first case by Lemma 2.5 and Corollary A.7.

If $l\left(s_{i} u\right)>l(u)$, then the root $\alpha:=u^{-1}\left(\alpha_{i}\right)$ is positive. Therefore,

$$
\mathrm{B}_{-} e_{i}(p) u \mathrm{~B}_{+}=\mathrm{B}_{-} u e_{\alpha}\left(p^{\prime}\right) \mathrm{B}_{+}=\mathrm{B}_{-} u \mathrm{~B}_{+} .
$$

Among all of the flags in (A.9), only $\mathrm{B}_{-} s_{i}$ is of Tits codistance $s_{i} u$ away from $\mathrm{B}_{-} u$, from which we arrive at the third case. The rest are of Tits codistance $u$, which proves the second case.

Now let us focus on $\mathrm{G}_{\mathrm{sc}}$. Let $V_{\lambda}$ denote the irreducible representation of $\mathrm{G}_{\mathrm{sc}}$ of highest weight $\lambda \in \mathrm{P}_{+}$. Let $\mathcal{O}\left[\mathrm{G}_{\mathrm{sc}}\right]$ be the algebra generated by the matrix coefficients of $V_{\lambda}, \lambda \in \mathrm{P}_{+}$.

Theorem A.10 (Kac-Peterson, [KP83, Theorem 1]). Consider the $\mathrm{G}_{\mathrm{sc}} \times \mathrm{G}_{\mathrm{sc}}$-action on $\mathcal{O}\left[\mathrm{G}_{\mathrm{sc}}\right]$ by

$$
\left(\left(g_{1}, g_{2}\right) . f\right)(g):=f\left(g_{1}^{-1} g g_{2}\right) .
$$

Then as $\mathrm{G}_{\mathrm{sc}} \times \mathrm{G}_{\mathrm{sc}}$-modules,

$$
\mathcal{O}\left[\mathrm{G}_{\mathrm{sc}}\right] \cong \bigoplus_{\lambda \in \mathrm{P}_{+}} V_{\lambda}^{*} \otimes V_{\lambda}
$$


Let ${ }^{\mathrm{U}_{-}} \mathcal{O}\left[\mathrm{G}_{\mathrm{sc}}\right]$ be the subring of left $\mathrm{U}_{-}$invariant functions. By Theorem A.10, we get

$$
\mathrm{U}_{-} \mathcal{O}\left[\mathrm{G}_{\mathrm{sc}}\right] \cong \bigoplus_{\lambda \in \mathrm{P}_{+}} V_{\lambda}
$$

Let $\Delta_{\lambda} \in V_{\lambda}$ be the unique highest weight vector such that $\Delta_{\lambda}(e)=1$. Given $\lambda, \mu \in \mathrm{P}_{+}$, the product $\Delta_{\lambda} \Delta_{\mu}$ is a highest weight vector in $V_{\lambda+\mu}$ and satisfies the normalisation condition. Therefore,

$$
\Delta_{\lambda+\mu}=\Delta_{\lambda} \Delta_{\mu}
$$

Theorem A.11 (Geiss-Leclerc-Schröer [GLS11], 7.2). An element $x \in \mathrm{G}_{\mathrm{sc}}$ is Gaussian decomposable if and only if $\Delta_{\omega_{i}}(x) \neq 0$ for all fundamental weights $\omega_{i}$.

\section{A.2. Basics about Cluster Algebras}

We include here the basic facts about cluster algebras that will be needed.

Definition A.12. A seed is a quadruple $\mathbf{s}=\left(I, I^{\mathrm{uf}}, \epsilon_{a b},\left\{d_{a}\right\}_{a \in I}\right)$ satisfying the following properties:

(1) $I$ is a finite set and $I^{\text {uf }} \subset I$;

(2) $\epsilon_{a b}$ is a $\mathbb{Q}$-valued matrix with $\epsilon_{a b} \in \mathbb{Z}$ unless $(a, b) \in I^{u f} \times I^{u f}$;

(3) $\left\{d_{a}\right\}$ is a collection of positive integers with $\operatorname{gcd}\left(d_{a}\right)=1$ such that the matrix $\hat{\epsilon}_{a b}:=\epsilon_{a b} d_{b}^{-1}$ is skew-symmetric.

Elements of $I$ are called vertices, elements of $I^{\mathrm{uf}}$ are called unfrozen vertices and elements of $I \backslash I^{\mathrm{uf}}$ are called frozen vertices. The matrix $\epsilon$ is called the exchange matrix and $d_{a}$ are called multipliers.

Definition A.13. Given a seed $\mathbf{s}$ and an unfrozen vertex $c \in I^{\mathrm{uf}}$, a mutation at $c$ produces a new seed $\mathbf{s}^{\prime}=\mu_{c} \mathbf{s}=\left(I^{\prime}, I^{\prime \text { uf }}, \epsilon_{a b}^{\prime},\left\{d_{a}^{\prime}\right\}\right)$ with $I^{\prime}=I, I^{\prime \text { uf }}=I^{\text {uf }}, d_{a}^{\prime}=d_{a}$ and

$$
\epsilon_{a b}^{\prime}= \begin{cases}-\epsilon_{a b} & \text { if } c \in\{a, b\}, \\ \epsilon_{a b}+\left[\epsilon_{a c}\right]_{+}\left[\epsilon_{c b}\right]_{+}-\left[-\epsilon_{a c}\right]_{+}\left[-\epsilon_{c b}\right]_{+} & \text {if } c \notin\{a, b\},\end{cases}
$$

where $[x]_{+}:=\max \{x, 0\}$. Seeds obtained by a sequence of mutations on $\mathbf{s}$ are said to be mutation equivalent to $\mathbf{s}$.

Let $\mathbb{T}$ be an $\left|I^{\mathrm{uf}}\right|$-regular tree. The edges of $\mathbb{T}$ are labelled by elements of $I^{\mathrm{uf}}$ such that the labelling of edges connecting to the same vertex are distinct. Every mutation is involutive: $\mu_{c}^{2} \mathbf{s}=\mathbf{s}$. Therefore, we can associate the vertices of $\mathbb{T}$ with seeds from a mutation equivalent family, such that any two vertices associated to a pair of seeds related by a mutation $\mu_{c}$ are joined by an edge labelled by $c$. The decorated tree $\mathbb{T}$ is called the mutation tree of $\mathbf{s}$.

We assign to each vertex $\mathbf{s}$ of $\mathbb{T}$ two split $|I|$-dimensional algebraic tori: a $\mathrm{K}_{2}$ seed torus $T_{\mathscr{A} ; \mathbf{s}}$ and a Poisson seed torus $T_{\mathscr{X} ; \mathbf{s}}$. The tori $T_{\mathscr{A} ; \mathbf{s}}$ and $T_{\mathscr{X} ; \mathbf{s}}$ are equipped with coordinate systems $\left\{A_{a ; \mathbf{s}}\right\}_{a \in I}$ and $\left\{X_{a ; \mathbf{s}}\right\}_{a \in I}$, respectively. We often drop the subscript ; $\mathbf{s}$ if it is obvious or not important. The torus $T_{\mathscr{A} ; \mathbf{s}}$ admits a canonical 2-form

$$
\Omega=\sum_{a, b} \hat{\epsilon}_{a b ; \mathbf{s}} \frac{d A_{a ; \mathbf{s}}}{A_{a ; \mathbf{s}}} \wedge \frac{d A_{b ; \mathbf{s}}}{A_{b ; \mathbf{s}}} .
$$

The torus $T_{\mathscr{X} ; \mathbf{s}}$ admits a canonical Poisson structure determined by the bivector field

$$
\Pi=\sum_{a, b} \hat{\epsilon}_{a b ; \mathbf{s}} X_{a ; \mathbf{s}} X_{b ; \mathbf{s}} \frac{\partial}{\partial X_{a ; \mathbf{s}}} \wedge \frac{\partial}{\partial X_{b ; \mathbf{s}}} .
$$


For every edge $\mathbf{s} \frac{c}{\mathbf{s}^{\prime}}$ in $\mathbb{T}$, the associated seed tori are related by the transition maps

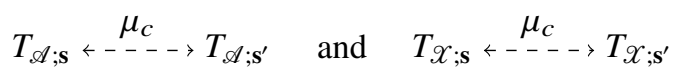

In terms of the cluster coordinates, the transition maps are expressed as

$$
\begin{gathered}
\mu_{c}^{*}\left(A_{a ; \mathbf{s}^{\prime}}\right):= \begin{cases}A_{c ; \mathbf{s}}^{-1}\left(\prod_{b} A_{b ; \mathbf{s}}^{\left[-\epsilon_{c b ;}\right]_{+}}\right)\left(1+\prod_{b} A_{b ; \mathbf{s}}^{\epsilon_{c b}}\right) & \text { if } a=c, \\
A_{a ; \mathbf{s}} & \text { if } a \neq c,\end{cases} \\
\mu_{c}^{*}\left(X_{a ; \mathbf{s}^{\prime}}\right):= \begin{cases}X_{c ; \mathbf{s}}^{-1} a=c, \\
X_{a ; \mathbf{s}} X_{c ; \mathbf{s}}^{\left[\epsilon_{a c ; s}\right]_{+}}\left(1+X_{c ; \mathbf{s}}\right)^{-\epsilon_{a c ; \mathbf{s}}} & \text { if } a \neq c .\end{cases}
\end{gathered}
$$

The maps $\mu_{c}$ preserve the 2-form $\Omega$ and the bivector field $\Pi$.

Let $\mathbf{s}$ and $\mathbf{s}^{\prime}$ be any two not necessarily adjacent vertices on $\mathbb{T}$. By composing the transition maps along the unique path connecting them, we get birational maps $\mu_{\mathbf{s} \rightarrow \mathbf{s}^{\prime}}: T_{\mathscr{A} ; \mathbf{s}} \rightarrow T_{\mathscr{A} ; \mathbf{s}^{\prime}}$ and $\mu_{\mathbf{s} \rightarrow \mathbf{s}^{\prime}}: T_{\mathscr{X} ; \mathbf{s}} \rightarrow T_{\mathscr{X} ; \mathbf{s}^{\prime}}$. Fomin and Zelevinsky [FZ07] proved the following factorisation formulas for the pullbacks of cluster coordinates:

$$
\begin{gathered}
\mu_{\mathbf{s} \rightarrow \mathbf{s}^{\prime}}^{*}\left(A_{a ; \mathbf{s}^{\prime}}\right)=\left(\prod_{b} A_{b ; \mathbf{s}}^{g_{a b ; s \rightarrow s^{\prime}}}\right)\left(\left.F_{a ; \mathbf{s} \rightarrow \mathbf{s}^{\prime}}\right|_{X_{b ; \mathbf{s}}=\prod_{c} A_{c ; \mathbf{s}} \epsilon_{b c ; s}}\right), \\
\mu_{\mathbf{s} \rightarrow \mathbf{s}^{\prime}}^{*}\left(X_{a ; \mathbf{s}^{\prime}}\right)=\left(\prod_{b} X_{b ; \mathbf{s}}^{c_{a b ; \mathbf{s} \rightarrow \mathbf{s}^{\prime}}}\right)\left(\prod_{b}\left(F_{b ; \mathbf{s} \rightarrow \mathbf{s}^{\prime}} \epsilon_{a b ; \mathbf{s}^{\prime}}\right),\right.
\end{gathered}
$$

where $c_{a b ; \mathbf{s} \rightarrow \mathbf{s}^{\prime}}$ and $g_{a b ; \mathbf{s} \rightarrow \mathbf{s}^{\prime}}$ are $I \times I$ matrices with integer entries and $F_{a ; \mathbf{s} \rightarrow \mathbf{s}^{\prime}}$ are polynomials in the initial cluster Poisson coordinates $\left\{X_{a ; s}\right\}$. They are called the c-matrix, the g-matrix and the $F$ polynomials associated to the mutation map $\mu_{\mathbf{s} \rightarrow \mathbf{s}^{\prime}}$, respectively. We have the following properties:

(1) (matrix identities) $\epsilon_{; \mathbf{s}^{\prime}} g_{; \mathbf{s} \rightarrow \mathbf{s}^{\prime}}=c_{; \mathbf{s} \rightarrow \mathbf{s}^{\prime}} \epsilon_{; \mathbf{s}}$;

(2) (sign coherence) row vectors of $c$-matrices and column vectors of $g$-matrices are sign coherent; that is, their entries are either all nonnegative or all nonpositive;

(3) (constant term) $F$-polynomials all have a constant term 1 ;

(4) (positivity) $F$-polynomials all have positive integer coefficients.

In addition, the following properties are immediate consequences of the factorisation formulas:

(5) (Laurent phenomenon) $\mu_{\mathbf{s} \rightarrow \mathbf{s}^{\prime}}\left(A_{a ; \mathbf{s}^{\prime}}\right)$ is a Laurent polynomial;

(6) $c_{a b ; \mathbf{s} \rightarrow \mathbf{s}^{\prime}}=\operatorname{ord}_{X_{b ; s}} \mu_{\mathbf{s} \rightarrow \mathbf{s}^{\prime}}^{*}\left(X_{a ; \mathbf{s}^{\prime}}\right)$, where ord $x f$ yields the lowest degree of $x$ in $f$ if $f$ is a polynomial and $\operatorname{ord}_{x}\left(\frac{f}{g}\right):=\operatorname{ord}_{x} f-\operatorname{ord}_{x} g$.

Let us fix an initial seed $\mathbf{s}$. The sign coherence of $c$-vectors allows us to assign a colour to each vertex $a \in I$ in a seed $\mathbf{s}^{\prime}$ : we say $a$ is green if $c_{a b ; \mathbf{s} \rightarrow \mathbf{s}^{\prime}} \geq 0$ for all $b$ and red otherwise. Note that a mutation at the vertex $c$ changes its colour, but it may change the colours of other vertices as well.

From the above definition, all vertices of the initial seed $\mathbf{s}$ are green. A sequence of mutations that turns all vertices red is called a reddening sequence and a reddening sequence consisting of mutations only in the direction of green vertices is called a maximal green sequence.

There is a combinatorial way to compute the $c$-matrix using principal coefficients. Given a seed $\mathbf{s}=\left(I, I^{\mathrm{uf}}, \epsilon_{; \mathbf{s}},\left\{d_{a}\right\}\right)$, we define the corresponding seed with principal coefficients as

$$
\mathbf{s}_{\text {prin }}=\left(I \sqcup I, I^{\mathrm{uf}} \sqcup \emptyset,\left(\begin{array}{cc}
\epsilon_{\text {; }} & \mathrm{id} \\
-\mathrm{id} & 0
\end{array}\right),\left\{d_{a}\right\} \sqcup\left\{d_{a}\right\}\right) .
$$


By applying the sequence of mutations $\mu_{\mathbf{s} \rightarrow \mathbf{s}^{\prime}}$ to $\mathbf{S}_{\text {prin }}$, we obtain a seed with exchange matrix $\left(\begin{array}{cc}\epsilon_{; \mathbf{s}^{\prime}} & c_{; \mathbf{s} \rightarrow \mathbf{s}^{\prime}} \\ * & *\end{array}\right)$, whose upper right-hand corner is precisely the $c$-matrix we need.

Definition A.18. Fix an initial seed $\mathbf{s}$ in $\mathbb{T}$. We define the upper cluster algebra to be

$$
\operatorname{up}(\mathscr{A}):=\bigcap_{\mathbf{s}^{\prime}} \mu_{\mathbf{s} \rightarrow \mathbf{s}^{\prime}}^{*}\left(\mathcal{O}\left(T_{\mathscr{A} ; \mathbf{s}^{\prime}}\right)\right) \subset \operatorname{Frac}\left(\mathcal{O}\left(T_{\mathscr{A} ; \mathbf{s}}\right)\right)
$$

and define the cluster Poisson algebra to be

$$
\operatorname{up}(\mathscr{X}):=\bigcap_{\mathbf{s}} \mu_{\mathbf{s} \rightarrow \mathbf{s}^{\prime}}^{*}\left(\mathcal{O}\left(T_{\mathscr{X} ; \mathbf{s}^{\prime}}\right)\right) \subset \operatorname{Frac}\left(\mathcal{O}\left(T_{\mathscr{X} ; \mathbf{s}}\right)\right) .
$$

Note that $\mathcal{O}\left(T_{\mathscr{X} ; \mathbf{s}^{\prime}}\right)$ is a Poisson algebra for each seed $\mathbf{s}^{\prime}$ and the mutation maps preserve the Poisson structure. Therefore, up $(\mathscr{X})$ is naturally a Poisson algebra.

The algebras up $(\mathscr{A})$ and up $(\mathscr{X})$ do not depend on the choice of an initial seed, because all mutation maps are algebra isomorphisms on the fields of fractions and $\mu_{c}^{2}=\mathrm{id}$.

Definition A.19. The geometric counterparts of up $(\mathscr{A})$ and up $(\mathscr{X})$ are the cluster $\mathrm{K}_{2}$ variety $\mathscr{A}$ and the cluster Poisson variety ${ }^{8} \mathscr{X}$, obtained by gluing the seed tori via the transition maps $\mu$ :

$$
\mathscr{A}=\bigsqcup_{\mathbf{s}} T_{\mathscr{A} ; \mathbf{s}} /\left\{\mu_{c}\right\} \quad \text { and } \quad \mathscr{X}=\bigsqcup_{\mathbf{s}} T_{\mathscr{X} ; \mathbf{s}} /\left\{\mu_{c}\right\} .
$$

In particular, $\mathcal{O}(\mathscr{A})=\operatorname{up}(\mathscr{A})$ and $\mathcal{O}(\mathscr{X})=\operatorname{up}(\mathscr{X})$.

Because the mutation maps between $T_{\mathscr{A} ; \mathbf{s}}$ preserve the canonical 2-forms $\Omega$, these 2-forms can be glued into a canonical 2 -form $\Omega$ on the cluster $\mathrm{K}_{2}$ variety $\mathscr{A}$. Similarly, because the mutation maps between $T_{\mathscr{X} ; \mathbf{s}}$ are Poisson maps, the cluster Poisson variety $\mathscr{X}$ is naturally a Poisson variety.

Definition A.20. Let $\mathbf{s}$ and $\mathbf{s}^{\prime}$ be two seeds on $\mathbb{T}$. A seed isomorphism $\sigma^{*}: \mathbf{s} \rightarrow \mathbf{s}^{\prime}$ is a bijection $\sigma^{*}: I \rightarrow I$ such that $\sigma^{*}\left(I^{\mathrm{uf}}\right)=I^{\mathrm{uf}}, d_{a}=d_{\sigma^{*}(a)}$ and $\epsilon_{a b ; \mathbf{s}}=\epsilon_{\sigma^{*}(a) \sigma^{*}(b) ; \mathbf{s}^{\prime}}$.

Definition A.21. A seed isomorphism $\sigma^{*}: \mathbf{s} \rightarrow \mathbf{s}^{\prime}$ induces algebra automorphisms $\sigma^{*}$ on up $(\mathscr{A})$ and up $(\mathscr{X})$ defined by

$$
\sigma^{*}\left(A_{a ; \mathbf{s}}\right):=A_{\sigma^{*}(a) ; \mathbf{s}^{\prime}} \quad \text { and } \quad \sigma^{*}\left(X_{a ; \mathbf{s}}\right):=X_{\sigma^{*}(a) ; \mathbf{s}^{\prime}} .
$$

Abusing notation, we still denote by $\sigma$ the induced biregular automorphisms of the corresponding cluster varieties. Such automorphisms are called cluster transformations.

A cluster transformation pulls back cluster variables to cluster variables according to the factorisation formulas (A.16) and (A.17). Therefore. it makes sense to define the $c$-matrix, the $g$-matrix and the $F$ polynomials of a cluster transformation $\sigma$ with respect to a choice of initial seed $\mathbf{s}$; we denote them by $c_{; \sigma ; \mathbf{s}}, g_{; \sigma ; \mathbf{s}}$ and $F_{; \sigma ; \mathbf{s}}$. respectively.

The following criteria determine when a cluster transformation is trivial.

Theorem A.22 ([GS18, GHKK18, CHL18]). Let $\sigma$ be a cluster transformation on $\mathscr{A}$ and $\mathscr{X}$. The following statements are equivalent:

○ $\sigma$ acts trivially on $\mathscr{A}$;

○ $\sigma$ acts trivially on $\mathscr{X}$;

$\circ$ the c-matrix with respect to one (and equivalently any) seed is the identity matrix;

$\circ$ the g-matrix with respect to one (and equivalently any) seed is the identity matrix.

${ }^{8}$ The cluster Poisson variety $\mathscr{X}$ is not a variety in the traditional sense because it often fails to be separated. 
By Theorem A.22, the group of cluster transformations on $\mathscr{A}$ coincides with the group of cluster transformations on $\mathscr{X}$. We call this group the cluster modular group and denote it by $\mathscr{G}$.

Definition A.23. Fix a seed $\mathbf{s}=\left(I, I^{\mathrm{uf}}, \epsilon_{a b},\left\{d_{a}\right\}\right)$. Let $d=1 \mathrm{~cm}\left\{d_{a}\right\}$. We define the Langlands dual seed $\mathbf{s}^{\vee}:=\left(I, I^{\mathrm{uf}},-\epsilon_{b a},\left\{d / d_{a}\right\}\right)$ and the chiral dual seed $\mathbf{s}^{\circ}:=\left(I, I^{\mathrm{uf}},-\epsilon_{a b},\left\{d_{a}\right\}\right)$.

The following facts are easy to check:

(a) $\mathbf{s}^{\mathrm{V} \vee}=\mathbf{s}$ and $\mathbf{s}^{\circ \circ}=\mathbf{s}$;

(b) $\mu_{c} \mathbf{s}^{\vee}=\left(\mu_{c} \mathbf{s}\right)^{\vee}$ and $\mu_{c} \mathbf{s}^{\circ}=\left(\mu_{c} \mathbf{s}\right)^{\circ}$;

(c) the following are equivalent for a bijection $\sigma^{*}: I \rightarrow I$ :

$\circ \sigma^{*}: \mathbf{s} \rightarrow \mathbf{s}^{\prime}$ is a seed isomorphism;

$\circ \sigma^{*}: \mathbf{s}^{\vee} \rightarrow \mathbf{s}^{\prime \vee}$ is a seed isomorphism;

$\circ \sigma^{*}: \mathbf{s}^{\circ} \rightarrow \mathbf{s}^{\prime \circ}$ is a seed isomorphism.

Thanks to (b), the mutation trees of $\mathbf{s}, \mathbf{s}^{\vee}$ and $\mathbf{s}^{\circ}$ are naturally isomorphic. We can define the Langlands dual versions and chiral dual versions of cluster algebras and cluster varieties the same way as before and we will denote them with superscripts ${ }^{\vee}$ and ${ }^{\circ}$, respectively. Nakanishi and Zelevinsky [NZ12] proved the following tropical duality relating the $c$-matrix and $g$-matrix associated to the same sequence of mutations when applied to Langlands dual seeds:

$$
c_{; \mathbf{s} \rightarrow \mathbf{s}^{\prime}}^{t}=g_{; \mathbf{s}^{\vee} \rightarrow \mathbf{s}^{\prime v}}^{-1}
$$

they also proved the following identity relating the $c$-matrices associated to the opposite sequence of mutations when applied to chiral dual seeds:

$$
c_{; \mathbf{s} \rightarrow \mathbf{s}^{\prime}}^{-1}=c_{; \mathbf{s}^{\prime \circ} \rightarrow \mathbf{s}^{\circ}} .
$$

It follows from (c) and the tropical duality that a cluster transformation is trivial if and only if the corresponding cluster transformation on the Langlands dual (respectively chiral dual) is trivial. Thus, the Langlands dual (respectively chiral dual) cluster modular groups are isomorphic; that is, $\mathscr{G} \cong \mathscr{G}^{\vee} \cong \mathscr{G}^{\circ}$.

Cluster varieties $\mathscr{A}$ and $\mathscr{X}$ are positive spaces; that is, they are equipped with a semifield of positive rational functions $\mathbb{Q}_{+}(\mathscr{A})$ and $\mathbb{Q}_{+}(\mathscr{X})$, respectively. Given a semifield $S$, we define the set of $S$-points

$$
\mathscr{A}(S):=\operatorname{Hom}_{\text {semifield }}\left(\mathbb{Q}_{+}(\mathscr{A}), S\right), \quad \mathscr{X}(S):=\operatorname{Hom}_{\text {semifield }}\left(\mathbb{Q}_{+}(\mathscr{X}), S\right) .
$$

Let $\mathbb{Z}^{t}=(\mathbb{Z}, \min ,+)$ be the semifield of tropical integers. Fock and Goncharov proposed the following conjecture on Langlands dual cluster varieties.

Conjecture A.26 ([FG09a, Conj. 4.1]). The coordinate ring $\mathcal{O}(\mathscr{A})$ admits a basis $\mathscr{G}$-equivariantly parametrised by $\mathscr{X}^{\vee}\left(\mathbb{Z}^{t}\right)$ and $\mathcal{O}(\mathscr{X})$ admits a basis $\mathscr{G}$-equivariantly parametrised by $\mathscr{A}^{\vee}\left(\mathbb{Z}^{t}\right)$.

Definition A.27. For a seed $\mathbf{s}=\left(I, I^{\mathrm{uf}}, \epsilon,\left\{d_{a}\right\}\right)$ with $\operatorname{gcd}\left\{d_{a}\right\}_{a \in I^{\text {uf }}}=1$, we define its associated unfrozen seed to be $\mathbf{s}^{\mathrm{uf}}=\left(I^{\mathrm{uf}}, I^{\mathrm{uf}},\left.\epsilon\right|_{I^{\mathrm{uf}} \times I^{\mathrm{uf}}},\left\{d_{a}\right\}_{a \in I^{\mathrm{uf}}}\right)$.

Let $\mathscr{A}^{\mathrm{uf}}$ and $\mathscr{X}^{\mathrm{uf}}$ be the cluster varieties constructed from an unfrozen seed associated to a seed that defines $\mathscr{A}$ and $\mathscr{X}$. Then among their seed tori we can define four maps

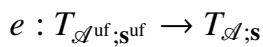

$$
\begin{aligned}
& f: T_{\mathscr{A}^{\mathrm{uf}} ; \mathbf{s}^{\mathrm{uf}}} \rightarrow T_{\mathscr{X} ; \mathbf{s}} \\
& e^{*}\left(A_{a ; \mathbf{s}}\right):= \begin{cases}A_{a ; \mathbf{s}^{\mathrm{uf}}} & \text { if } a \in I^{\mathrm{uf}}, \\
1 & \text { if } a \notin I^{\mathrm{uf}},\end{cases} \\
& f^{*}\left(X_{a ; \mathbf{s}}\right):=\prod_{b \in I^{\text {uf }}} A_{b ; \mathbf{s}^{\mathbf{u f}^{\prime}}}^{\epsilon_{a b ; \mathrm{s}}} \\
& p: T_{\mathscr{A} ; \mathbf{s}} \rightarrow T_{\mathscr{X}}{ }_{\text {uf } ; \mathbf{s}^{\mathrm{uf}}} \\
& p^{*}\left(X_{a ; \mathbf{s}^{\mathrm{uf}}}\right):=\prod_{b \in I} A_{b ; \mathbf{s}}^{\epsilon_{a b ; \mathrm{s}}} \\
& q: T_{\mathscr{X} ; \mathbf{s}} \rightarrow T_{\mathscr{X}^{\mathrm{uf} ; \mathbf{s}^{\mathrm{uf}}}} \\
& q^{*}\left(X_{a ; \mathbf{s}^{\mathrm{uf}}}\right):=X_{a ; \mathbf{s}} \text {. }
\end{aligned}
$$


By direct computation one can verify that these four maps commute with the mutation maps $\mu$. Therefore, we can glue them together and obtain four regular maps in the following commutative diagram:

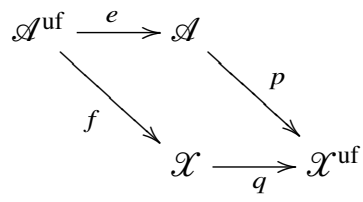

Let $\mathscr{G}$ be the cluster modular group associated to the cluster varieties $\mathscr{A}$ and $\mathscr{X}$ and let $\mathscr{G}^{\text {uf }}$ be the cluster modular group associated to the cluster varieties $\mathscr{A}^{\text {uf }}$ and $\mathscr{X}^{\text {uf }}$. It then follows from the above formulas that $\mathscr{G}$ is a subgroup of $\mathscr{G}^{\text {uf }}$.

Proposition A.28. Let $\pi: T_{\mathscr{A} ; \mathbf{s}} \rightarrow T_{\mathscr{X} ; \mathbf{s}}$ be a group homomorphism of algebraic tori for some fixed seed $\mathbf{s}$ such that $f=\pi \circ e$ and $p=q \circ \pi$. Then $\pi$ induces group homomorphisms of algebraic tori between seed tori associated to any other seed in the mutation equivalent family. Moreover, they glue into a well-defined regular map $\pi: \mathscr{A} \rightarrow \mathscr{X}$.

Proof. The conditions $p=\pi \circ e$ and $f=q \circ \pi$ imply that the only freedom in defining $\pi$ is the frozen factor (the first factor) in

$$
\pi^{*}\left(X_{a ; \mathbf{s}}\right):=\left(\prod_{b \in I \backslash I^{\mathrm{uf}}} A_{b ; \mathbf{s}}^{\cdots}\right)\left(\prod_{c \in I^{\mathrm{uf}}} A_{c ; \mathbf{s}}^{\epsilon_{a c ; \mathrm{s}}}\right)=\left(\prod_{b \in I \backslash I^{\mathrm{uf}}} A_{b ; \mathbf{s}}^{\cdots}\right) f^{*}\left(X_{a ; \mathbf{s}}\right)
$$

for each frozen vertex $a$. But such a factor need not change under mutation: we can just define $\pi^{*}\left(X_{a ; \mathbf{s}^{\prime}}\right):=$ $\left(\prod_{b \in I \backslash I^{\text {uf }}} A_{b ; \mathbf{s}^{\prime}}^{\ldots}\right) f^{*}\left(X_{a ; \mathbf{s}^{\prime}}\right)$ for any other seed $\mathbf{s}^{\prime}$ and such maps $\pi$ automatically commute with the mutation maps.

Remark A.29. There is another way to describe the map $\pi$. Let $M$ and $N$ be the character lattices of $T_{\mathscr{A} ; \mathbf{s}}$ and $T_{\mathscr{X} ; \mathbf{s}}$, respectively. Then $\pi$ induces a linear map of character lattices $\pi^{*}: N \rightarrow M$. The cluster Poisson coordinates $\left\{X_{a ; \mathbf{s}}\right\}$ correspond to a basis $\left\{e_{a ; \mathbf{s}}\right\}$ of $N$ and the skew-symmetric matrix $\hat{\epsilon}_{a b}$ defines a skew-symmetric form $\{\cdot, \cdot\}$ on $N$. We can view a seed mutation $\mathbf{s}^{\prime}=\mu_{c} \mathbf{s}$ as a change of basis on the lattice $N$ given by

$$
e_{a ; \mathbf{s}^{\prime}}= \begin{cases}-e_{c ; \mathbf{s}} & \text { if } a=c, \\ e_{a ; \mathbf{s}}+\left[\epsilon_{a c ; \mathbf{s}}\right]_{+} e_{c ; \mathbf{s}} & \text { if } a \neq c .\end{cases}
$$

By viewing the seed tori $T_{\mathscr{A} ; \mathbf{s}^{\prime}}$ and $T_{\mathscr{X} ; \mathbf{s}^{\prime}}$ as algebraic tori with character lattices $M$ and $N$, we can redefine the mutation map $\mu_{c}$ between the corresponding seed tori by the following coordinate-free formula:

$$
\begin{gathered}
\mu_{c}^{*}\left(X^{n}\right)=X^{n}\left(1+X^{e_{c ; s}}\right)^{-\left\{n, d_{c} e: c ; \mathbf{s}\right\}}, \\
\mu_{c}^{*}\left(A^{m}\right)=A^{m}\left(1+A^{p^{*}\left(e_{c ; s}\right)}\right)^{-\left\langle m, d_{c} e: c ; \mathbf{s}\right\rangle},
\end{gathered}
$$

where $X^{n}$ is the character function corresponding to $n \in N$ and $A^{m}$ is the character function corresponding to $m \in M$. By using this description, it is not hard to verify by computation that the following diagram commutes:

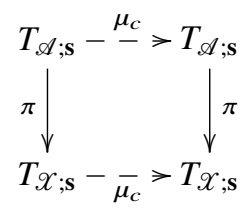

where the two vertical maps $\pi$ are induced by the same linear map $\pi^{*}: N \rightarrow M$. 
Proposition A.30. If in addition to the assumption of Proposition A.28 the map $\pi: T_{\mathscr{A} ; \mathbf{s}} \rightarrow T_{\mathscr{X} ; \mathbf{s}}$ is surjective, then the resulting regular map $\pi: \mathscr{A} \rightarrow \mathscr{X}$ is also surjective (and equivalently the induced algebra homomorphism $\pi^{*}: \operatorname{up}(\mathscr{X}) \rightarrow \operatorname{up}(\mathscr{A})$ is injective) and $F \in \operatorname{Frac}(\operatorname{up}(\mathscr{X}))$ is in up $(\mathscr{X})$ if and only if $\pi^{*}(F)$ is in $\operatorname{up}(\mathscr{A})$.

Proof. If $\pi: T_{\mathscr{A} ; \mathbf{s}} \rightarrow T_{\mathscr{X} ; \mathbf{s}}$ is surjective for one seed $\mathbf{s}$, then $\pi^{*}: N \rightarrow M$ is injective, which implies that the induced maps $\pi: T_{\mathscr{A} ; \mathbf{s}^{\prime}} \rightarrow T_{\mathscr{X} ; \mathbf{s}^{\prime}}$ are surjective for any seed $\mathbf{s}^{\prime}$ in the mutation equivalent family by the above observation. Then it follows that $\pi: \mathscr{A} \rightarrow \mathscr{X}$ is also surjective.

Lastly, because $\pi^{*}: T_{\mathscr{A}, \mathbf{s}} \rightarrow T_{\mathscr{X}, \mathbf{s}}$ maps monomials to monomials for every $\mathbf{s}, F$ is a Laurent polynomial on $T_{\mathscr{X}, \mathbf{s}}$ for all $\mathbf{s}$ if and only if $\pi^{*}(F)$ is a Laurent polynomial on $T_{\mathscr{A}, \mathbf{s}}$ for all $\mathbf{s}$, which implies that $F \in$ up $(\mathscr{X})$ if and only if $\pi^{*}(F) \in(\mathscr{A})$.

Next we will make use of the alternative description of a seed in Remark A.29 to define quasi-cluster transformations. A more detailed discussion can be found in [Fra16, GS19].

Definition A.31. Let $\mathbf{s}$ and $\mathbf{s}^{\prime}$ be two seeds on $\mathbb{T}$. Let $N$ be the lattice as described in Remark A.29, with a skew-symmetric form $\{\cdot, \cdot\}$. Let $\left\{e_{a ; \mathbf{s}}\right\}$ and $\left\{e_{a ; \mathbf{s}^{\prime}}\right\}$ be the bases of $N$ corresponding to the seeds $\mathbf{S}$ and $\mathbf{s}^{\prime}$, respectively. Then a lattice isomorphism $\sigma^{*}: N \rightarrow N$ is said to be a seed quasi-isomorphism if

(1) there exists a seed isomorphism $\sigma^{*}: \mathbf{s}^{\text {uf }} \rightarrow \mathbf{s}^{\text {'uf }}$ between the unfrozen seeds such that $\sigma^{*}\left(e_{a ; \mathbf{s}}\right)=$ $e_{\sigma(a) ; \mathbf{s}^{\prime}}$ for all $a \in I^{\mathrm{uf}}$

(2) $\sigma$ preserves the skew-symmetric form $\{\cdot, \cdot\}$ on $N$.

Note that a seed quasi-isomorphism $\sigma^{*}$ also induces a lattice isomorphism $M \rightarrow M$ because $M$ is dual to $N$ up to a rescaling. ${ }^{9}$ We abuse notation and denote the induced automorphism on $M$ by $\sigma^{*}$.

Definition A.32. A seed quasi-isomorphism $\sigma^{*}$ naturally induces an automorphism $\sigma^{*}$ on up $(\mathscr{A})$ and up $(\mathscr{X})$ defined by

$$
\sigma^{*}\left(A_{; \mathbf{s}}^{m}\right):=A_{; \mathbf{s}^{\prime}}^{\sigma^{*}(m)} \quad \text { and } \quad \sigma^{*}\left(X_{; \mathbf{s}}^{n}\right):=X_{; \mathbf{s}^{\prime}}^{\sigma^{*}(n)}
$$

we call such automorphisms quasi-cluster transformations. In turn, such automorphisms also define biregular automorphisms on the corresponding cluster varieties, which we also call quasi-cluster transformations. Because a seed quasi-isomorphism preserves the skew-symmetric form $\{\cdot, \cdot\}$, a quasi-cluster transformation on $\operatorname{up}(\mathscr{X})$ is automatically a Poisson automorphism.

Lemma A.33. Let $\sigma$ be a quasi-cluster transformation corresponding to a lattice isomorphism $\sigma^{*}$ between two seeds $\mathbf{s}$ and $\mathbf{s}^{\prime}$. Suppose that $m$ and $n$ are two integer matrices such that

$$
\sigma^{*}\left(A_{i ; \mathbf{s}}\right)=\prod_{j} A_{j ; \mathbf{s}^{\prime}}^{m_{i j}} \quad \text { and } \quad \sigma^{*}\left(X_{i ; \mathbf{s}}\right)=\prod_{j} X_{j ; \mathbf{s}^{\prime}}^{n_{i j}}
$$

Then $m d^{\prime}=d\left(n^{t}\right)^{-1}$, where $d$ and $d^{\prime}$ are the multiplier matrices associated with the seeds $\mathbf{s}$ and $\mathbf{s}^{\prime}$, respectively, and tenotes the transposition of a matrix.

Proof. By definition, we have $\sigma^{*}\left(e_{i}\right)=\sum_{j} n_{i j} e_{j}^{\prime}$ for the linear isomorphism $\sigma^{*}: N \rightarrow N$. On the other hand, we know from [GHKK18, Appendix A] that the two $f$-bases of $M$ are given by $f_{i}=e_{i}^{*} / d_{i}$ and $f_{j}^{\prime}=e_{j}^{\prime *} / d_{j}^{\prime}$, respectively. It follows from linear algebra that the induced map $\sigma^{*}: M \rightarrow M$ is given by

$$
\sigma^{*}\left(f_{i}\right)=\sum_{j} d_{i}^{-1}\left(n^{-1}\right)_{j i} d_{j}^{\prime} f_{j}^{\prime}
$$

Because every cluster transformation is a quasi-cluster transformation, the quasi-cluster transformations form a group that contains the cluster modular group $\mathscr{G}$.

${ }^{9}$ The rescaling is nontrivial only in the skew-symmetrisable cases; see [GHKK18, Appendix A] for more details. 
Example A.34. Below is a simple example that demonstrates the difference between a cluster transformation and a quasi-cluster transformation. Consider the following quivers, which differ by one single mutation:

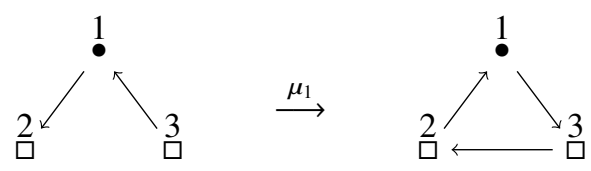

Note that these two quivers are not isomorphic, so they cannot possibly define a cluster transformation. On the other hand, if we define

$$
\sigma^{*}\left(e_{1}\right):=e_{1}^{\prime}, \quad \sigma^{*}\left(e_{2}\right):=e_{1}^{\prime}+e_{3}^{\prime}, \quad \sigma^{*}\left(e_{3}\right):=e_{2}^{\prime},
$$

we see that $\sigma^{*}$ defines a seed quasi-isomorphism. Therefore, $\sigma^{*}$ induces a quasi-cluster transformation on $\operatorname{up}(\mathscr{A})$ and $\operatorname{up}(\mathscr{X})$ that acts by

$$
\begin{array}{ll}
\sigma^{*}\left(X_{1}\right)=X_{1}^{\prime}=\frac{1}{X_{1}} & \sigma^{*}\left(A_{1}\right)=\frac{A_{1}^{\prime}}{A_{3}^{\prime}}=\frac{A_{2}+A_{3}}{A_{1} A_{3}} \\
\sigma^{*}\left(X_{2}\right)=X_{1}^{\prime} X_{3}^{\prime}=\frac{X_{3}}{1+X_{3}} & \sigma^{*}\left(A_{2}\right)=A_{3}^{\prime}=A_{3} \\
\sigma^{*}\left(X_{3}\right)=X_{2}^{\prime}=X_{2}\left(1+X_{1}\right) & \sigma^{*}\left(A_{3}\right)=A_{2}^{\prime}=A_{2} .
\end{array}
$$

The cluster Poisson algebra up ( $\left.\mathscr{X}^{\text {uf }}\right)$ admits a natural extension into a Poisson algebra of formal series. Goncharov and Shen defined a unique Poisson automorphism on such extension called the DonaldsonThomas transformation. In all known cases the Donaldson-Thomas transformation preserves up $\left(\mathscr{X}^{\mathrm{uf}}\right)$ and hence descends to a Poisson automorphism on up $\left(\mathscr{X}^{\mathrm{uf}}\right)$. In most known cases the DonaldsonThomas transformation is a central element in the cluster modular group $\mathscr{G}^{\mathrm{uf}}$, and when this happens we say that the Donaldson-Thomas transformation is cluster.

In the reverse direction, there is an easy way to check whether a cluster transformation is the Donaldson-Thomas transformation by using the $c$-matrix, and we will use it as the working definition in this article.

Definition A.35. A cluster transformation in $\mathscr{G}^{\mathrm{uf}}$ is the cluster Donaldson-Thomas transformation if its $c$-matrix (with respect to any choice of seed) is $-\mathrm{id}$.

The following theorem justifies the omission of the phrase 'with respect to any choice of seed'.

Theorem A.36 ([GS18, Theorem 3.6]). If $\sigma$ is a cluster transformation with $c_{; \sigma ; \mathrm{s}}=-\mathrm{id}$, then $c_{; \sigma ; \mathbf{s}^{\prime}}=$ -id for any seed $\mathbf{s}^{\prime}$ mutation equivalent to $\mathbf{s}$.

Recall that the $c$-matrix of a sequence of mutations can be computed using principal coefficients. It follows by definition of the cluster Donaldson-Thomas transformation that any two isomorphic seeds that give rise to the cluster transformation are related by a reddening sequence. On the the other hand, it is known that a reddening sequence always produces a seed that is isomorphic to the original seed and one can choose an isomorphism such that the associated $c$-matrix is -id. Therefore, we have the following implications:

a maximal green sequence exists

a reddening sequence exists

$$
\Downarrow
$$

Donaldson-Thomas transformation is rational transformation is cluster 
Let us explain the importance of Donaldson-Thomas transformations. First, in the skew-symmetric case (where all $d_{a}=1$ ), the Donaldson-Thomas transformation encodes the Donaldson-Thomas invariants of an associated 3D Calabi-Yau category of dg modules (see [GS18]). Second, by combining results of Goncharov and Shen [GS18] and results of Gross et al. [GHKK18], one obtains the following sufficient conditions for the cluster duality conjecture.

Theorem A.38 ([GHKK18, Prop.8.28]). Conjecture A.26 holds if the following two conditions hold:

(1) The Donaldson-Thomas transformation of $\mathscr{X}^{\mathrm{uf}}$ is a cluster.

(2) The algebra homomorphism $p^{*}:$ up $\left(\mathscr{X}^{\mathrm{uf}}\right) \rightarrow$ up $(\mathscr{A})$ is injective.

\section{A.3. Python Code for Computing $g_{d}^{b}(q)$ for Positive Braid Closures}

from sympy import *

$\mathrm{q}=\operatorname{symbols}($ ' q')

\# The Weyl group associated to Dynkin type A_(n-1) is the symmetric

\# group S_n. We denote elements of S_n by an $n$-tuple $u$ consisting of

\# integers from $\theta$ to $n$. Below are left/right multiplication of $u$ by

\# a simple reflection s_k

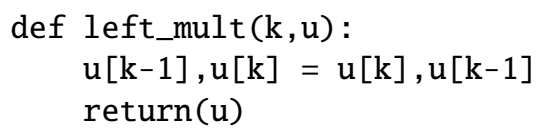

\# Below is an algorithm that converts an integer $\theta<=m<2^{\wedge} 1$ into an 1

\# tuple of $\theta \mathrm{s}$ and $1 \mathrm{~s}$ corresponding to the binary expansion of $\mathrm{m}$.

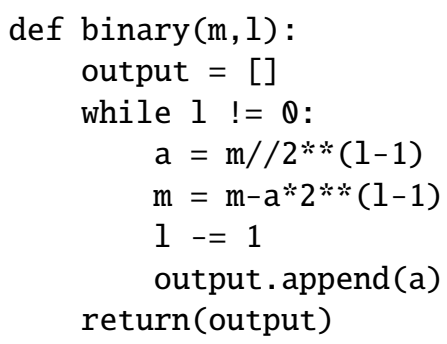

\# Now we are ready to define the function $g^{\wedge} b \_d(q)$.

\# The computation needs the input of a triangulation $t$. However,

\# the output should not depend on $t$.

\# Let $1=1(b)+1(d)$ 
\# The input of $b$ and $d$ will specify the choice of words. We then \# represent a triangulation $t$ by an 1-tuple of $0 \mathrm{~s}$ and $1 \mathrm{~s}$, with $\theta$ \# representing a nabla-shaped triangle and 1 representing a Delta\# shaped triangle.

$\operatorname{def} g(n, b, d, t)$ :

\# First we should test that $t$ is actually a triangulation for $(b, d)$.

if $t$. count ( $\theta) !=\operatorname{len}(b)$ or $t$. $\operatorname{count}(1) !=\operatorname{len}(d)$ : return("Error: Wrong Triangulation!")

\# Next we combine b and d into a 1-tuple "braid" of pairs ( $\mathrm{s}, \mathrm{k})$, \# where $s=0,1$ indicates the orientation of the triangle

\# and $\mathrm{k}$ indicates the simple reflection.

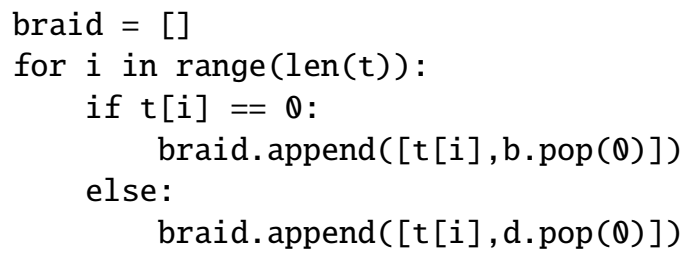

\# The variable "polynomial" will be the final output $g^{\wedge} b \_d(q) *(q-1)^{\wedge}(n-1)$

polynomial $=0$

\# When we go across the triangulation from left to right, at each

\# step we can either multiply or not multiply s_k.

\# Therefore at most we only need to consider $2^{\wedge} 1$ number of cases.

\# We go through all these cases using a parameter $0<=m<2^{\wedge} 1$

\# By converting this parameter $m$ into a binary expression of length 1 ,

\# we exhaust all possible cases.

\# 1 means the multiplication takes place,

\# and 0 means the multiplication does not take place.

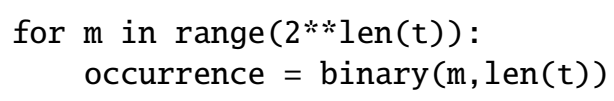

\# Start with the leftmost edge being of Tits codistance u=e.

$$
u=[j \text { for } j \text { in } \operatorname{range}(n)]
$$

\# The 1-tuple "factors" collects the factors coming from each

\# triangle in the triangulation.

$$
\text { factor }=[]
$$

\# We now go from left to right across the triangulation and

\# impose Tits codistance conditions $\mathrm{u}$ according to the binary 
[BFZ05] A. Berenstein, S. Fomin and A. Zelevinsky, 'Cluster algebras. III. Upper bounds and double Bruhat cells', Duke Math. J. 126(1) (2005), 1-52. doi:10.1215/S0012-7094-04-12611-9.

[BS58] R. Bott and H. Samelson, 'Applications of the theory of Morse to symmetric spaces', Amer. J. Math. 80 1958, 964-1029. doi:10.2307/2372843.

[CHL18] P. Cao, M. Huang and F. Li, 'A conjecture on c-matrices of cluster algebras', Nagoya Math. J. 238 (2020), $37-46$. doi:10.1017/nmj.2018.18.

[Dem74] M. Demazure, 'Désingularisation des variétés de Schubert généralisées', Ann. Sci. École Norm. Sup. (4), 7 (1974), 53-88.

[EL19] B. Elek and J.-H.Lu, 'Bott-Samelson varieties and Poisson ore extensions', Int. Math. Res. Not.. Preprint, 2019, arXiv:1601.00047.

[FG06] V. Fock and A. Goncharov, 'Cluster $X$-varieties, amalgamation, and Poisson-Lie groups', in Algebraic Geometry and Number Theory, Vol. 253 of Progr. Math. (Birkhäuser Boston, Boston, 2006). 27-68.

[FG09a] V. Fock and A. Goncharov, 'Cluster ensembles, quantization and the dilogarithm', Ann. Sci. Éc. Norm. Supér. (4) 42(6) (2009), 865-930. doi:10.24033/asens. 2112.

[FG09b] V. Fock and A. Goncharov, 'The quantum dilogarithm and representations of quantum cluster varieties', Invent. Math. 175(2) (2009), 223-286. doi: 10.1007/s00222-008-0149-3.

[FZ99] S. Fomin and A. Zelevinsky, 'Double Bruhat cells and total positivity', J. Amer. Math. Soc. 12(2) (1999), 335-380. doi:10.1090/S0894-0347-99-00295-7.

[FZ02] S. Fomin and A. Zelevinsky, 'Cluster algebras. I. Foundations', J. Amer. Math. Soc. 15(2) (2002), 497-529. doi:10.1090/S0894-0347-01-00385-X.

[FZ03] S. Fomin and A. Zelevinsky, ' $Y$-systems and generalized associahedra', Ann. Math. (2) 158(3) (2003), 977-1018. doi:10.4007/annals.2003.158.977.

[FZ07] S. Fomin and A. Zelevinsky, 'Cluster algebras. IV. Coefficients', Compos. Math. 143(1) (2007), $112-164$. doi:10.1112/S0010437X06002521.

[Fra16] C. Fraser, 'Quasi-homomorphisms of cluster algebras', Adv. Appl. Math. 81 (2016), 40-77. doi::10.1016/j.aam.2016.06.005.

[GL20] P. Galashin and T. Lam, 'Positroids, knots, and $q$, $t$-Catalan numbers'. Preprint, 2020, arXiv:2012.09745.

[GLS11] C. Geiß, B. Leclerc and J. Schröer, 'Kac-Moody groups and cluster algebras', Adv. Math. 228(1) (2011), 329-433. doi:10.1016/j.aim.2011.05.011.

[GSV10] M. Gekhtman, M. Shapiro and A. Vainshtein. Cluster Algebras and Poisson Geometry, Vol. 167 of Mathematical Surveys and Monographs (American Mathematical Society, Providence, RI, 2010). doi:10.1090/surv/167.

[GS18] A. Goncharov and L. Shen, 'Donaldson-Thomas transformations of moduli spaces of G-local systems', Adv. Math. 327 (2018), 225-348. doi:10.1016/j.aim.2017. 06.017.

[GS19] A. Goncharov and L. Shen, 'Quantum geometry of moduli spaces of local systems and representation theory', Preprint, 2019, arXiv:1904.10491.

[GY18] K. Goodearl and M. Yakimov, 'Cluster algebra structures on Poisson nilpotent algebras'. Preprint, 2018, arXiv:1801.01963.

[GHKK18] M. Gross, P. Hacking, S. Keel and M. Kontsevich, 'Canonical bases for cluster algebras', J. Amer. Math. Soc. 31(2) (2018), 497-608. doi:10.1090/jams/890.

[GKS12] S. Guillermou, M. Kashiwara and P. Schapira, 'Sheaf quantization of Hamiltonian isotopies and applications to nondisplaceability problems', Duke Math. J. 161(2) (2012), 201-245. doi:10.1215/00127094-1507367.

[Han73] H. C. Hansen, 'On cycles in flag manifolds', Math. Scand. 33 (1974), 269-274. doi:10.7146/math.scand.a-11489.

[Kac83] V. G. Kac. Infinite-Dimensional Lie Algebras, Vol. 44 of Progress in Mathematics (Birkhäuser Boston, Boston, 1983). doi:10.1007/978-1-4757-1382-4.

[KP83] V. G. Kac and D. H. Peterson, 'Regular functions on certain infinite-dimensional groups', in Arithmetic and Geometry, Vol. II, Vol. 36 of Progr. Math. (Birkhäuser Boston, Boston, 1983), 141-166. wwwmath.mit.edu/kac/not-easily-available/regularfunctions.pdf.

[Kel11] B. Keller, 'On cluster theory and quantum dilogarithm identities', in Representations of Algebras and Related Topics, EMS Ser. Congr. Rep. (European Mathematical Society, Zürich, Switzerland, 2011), 85-116. doi:10.4171/101-1/3.

[Kel13] B. Keller, 'The periodicity conjecture for pairs of Dynkin diagrams', Ann. Math. (2) 177(1) (2013), 111-170. doi:10.4007/annals.2013.177.1.3.

[KS08] M. Kontsevich and Y. Soibelman, 'Stability structures, motivic Donaldson-Thomas invariants and cluster transformations'. Preprint, 2008, arXiv:0811.2435.

[Kum02] S. Kumar, Kac-Moody Groups, Their Flag Varieties and Representation Theory, Vol. 204 of Progress in Mathematics (Birkhäuser Boston, Boston, 2002). doi:10.1007/978-1-4612-0105-2.

[LS16] T. Lam and D. Speyer, 'Cohomology of cluster varieties. I. Locally acyclic case'. Preprint, 2016, arXiv:1604.06843.

[LM17] J.-H. Lu and V. Mouquin, 'On the $T$-leaves of some Poisson structures related to products of flag varieties', $A d v$. Math. 306 (2017), 1209-1261. doi:10.1016/j.aim.2016.11.008.

[Mou19] V. Mouquin, 'Local Poisson groupoids over mixed product Poisson structures and generalised double Bruhat cells'. Preprint, 2019, arXiv:1908.04044. 
[Mul14] G. Muller, ' $\mathcal{A}=\mathcal{U}$ for locally acyclic cluster algebras', SIGMA 10 (2014), Paper 094. doi:10.3842/SIGMA. 2014.094.

[NZ12] T. Nakanishi and A. Zelevinsky, 'On tropical dualities in cluster algebras', in Algebraic Groups and Quantum Groups, Vol. 565 of Contemp. Math. (Amer. Math. Soc., Providence, RI, 2012), 217-226. doi:10.1090/conm/565/ 11159.

[SW20] L. Shen and D. Weng, 'Cyclic sieving and cluster duality for Grassmannian', SIGMA 16 (2020).

[STWZ19] V. Shende, D. Treumann, H. Williams and E. Zaslow, 'Cluster varieties from Legendrian knots', Duke Math. J. 168(15) (2019), 2801-2871. doi:10.1215/00127094-2019-0027.

[STZ17] V. Shende, D. Treumann and E. Zaslow, 'Legendrian knots and constructible sheaves', Invent. Math. 207(3) (2017), 1031-1133. doi:10.1007/s00222-016-0681-5.

[WY07] B. Webster and M. Yakimov, 'A Deodhar-type stratification on the double flag variety', Transform. Groups 12(4) (2007), 769-785. doi:10.1007/s00031-007-0061-8.

[Wen20] D. Weng, 'Donaldson-Thomas transformation of double Bruhat cells in semisimple Lie groups', Ann. Sci. Éc. Norm. Supér. 53 (2020), 291-352.

[Wen21] D. Weng, 'Donaldson-Thomas transformation of Grassmannian', Adv. Math. 383 (2021), 107721. doi:10.1016/j.aim.2021.107721.

[Wil13] H. Williams, 'Cluster ensembles and Kac-Moody groups', Adv. Math. 247 (2013), 1-40. doi:10.1016/j.aim. 2013.07.008.

[Zel00] A. Zelevinsky, 'Connected components of real double Bruhat cells', Internat. Math. Res. Not. 21 (2000), 1131-1154. doi: $10.1155 /$ S1073792800000568. 